\title{
Asymmetric Transfer Hydrogenation of Ketones Using New Iron(II) (P-NH-N-P’) Catalysts: Changing the Steric and Electronic Properties at Phosphorus P'
}

\author{
Samantha A. M. Smith, Demyan E. Prokopchuk, Alan J, Lough, Robert H. Morris
}

Version Post-print/accepted manuscript

Citation S. A. M. Smith, D. E. Prokopchuk, A. J. Lough, R. H. Morris, Israel (published version) J. Chem. 2017, 57, 1204-1215.

http://doi.org/10.1002/ijch.201700019

\section{Copyright / License}

Publisher's Statement
This document is the Accepted Manuscript version of a Published Work that appeared in final form in Israel Journal of Chemistry, copyright (C) Wiley-VCH after peer review and technical editing by the publisher. To access the final edited and published work see http://doi.org/10.1002/ijch.201700019

This article was made openly accessible by $U$ of 'T Faculty. Please tell us how this access benefits you. Your story matters. 


\section{TSpace Research Repository}

because you cannot access the published version, then cite the TSpace version in addition to the published version using the permanent URI (handle) found on the record page. 


\section{Asymmetric Transfer Hydrogenation of}

\section{Ketones Using New Iron(II) (P-NH-N-P')}

\section{Catalysts: Changing the Steric and}

\section{Electronic Properties at Phosphorus $P^{\prime}$}

Samantha A. M. Smith, Demyan, E. Prokopchuk and Robert H. Morris*

Department of Chemistry, University of Toronto, 80 Saint George St. Toronto, Ont. Canada M5S 3H6

\section{rmorris@chem.utoronto.ca}

Abstract. The asymmetric transfer hydrogenation (ATH) of ketones is an efficient method for producing enantio-enriched alcohols which are used as intermediates in a variety of industrial processes. Here we report the synthesis of new iron ATH precatalysts $(S, S)-\left[\mathrm{FeBr}(\mathrm{CO})\left(\mathrm{Ph}_{2} \mathrm{PCH}_{2} \mathrm{CH}_{2} \mathrm{NHCHPh}^{-}\right.\right.$ $\left.\left.\mathrm{CHPhN}=\mathrm{CHCH}_{2} \mathrm{PR}_{2}{ }_{2}\right)\right]\left[\mathrm{BPh}_{4}\right]\left(\mathrm{R}^{\prime}=\mathrm{Et}\right.$, and ortho-tolyl) where one of the phosphine groups is modified with a small alkyl and a large aryl substituent to probe the effect of this change on the activity and selectivity of the catalytic system. A simple reversible equilibrium kinetic model is used to obtain the initial TOF and the inherent enantioselectivity $S=k_{R} / k_{S}$ of these catalysts along with those for the previously reported catalysts with $\mathrm{R}^{\prime}=\mathrm{Ph}$ and $\mathrm{Cy}$ for the $\mathrm{ATH}$ of acetophenone. With an increase in the size of the $\mathrm{PR}_{2}{ }_{2}$ group, the TOF goes through a maximum at $\mathrm{PPh}_{2}$ while the $\mathrm{S}$ value goes through a maximum of 510 at $\mathrm{R}^{\prime}=\mathrm{Cy}$. The complex with $\mathrm{R}^{\prime}=o$-Tol starts with a high $S$ value of 200 but is rapidly changed to a second catalyst with an $S$ value of 28 . For the reduction of acetophenone to $(R)$-1-phenylethanol, turnover numbers of up to 5200 and ee up to $98 \%$ were achieved. The chemotherapeutic pharmaceutical precursor $(R)-\left(3^{\prime}, 5^{\prime}-\right.$ bis(trifluoromethyl))-1-phenylethanol is synthesized in up to $95 \%$ ee. Several other alcohols can be prepared in greater than $90 \%$ ee by choosing the precatalyst with the correctly matched steric properties. A hydride complex derived from the catalyst with $R^{\prime}=C y$ is characterized by NMR spectroscopy. It is proposed that low concentration trans-hydride carbonyl complexes with the FeH parallel to the $\mathrm{NH}$ of the ligand are the active catalysts in all of these systems. 
Keywords: homogeneous catalysis, iron catalysis, asymmetric transfer hydrogenation

Biographies

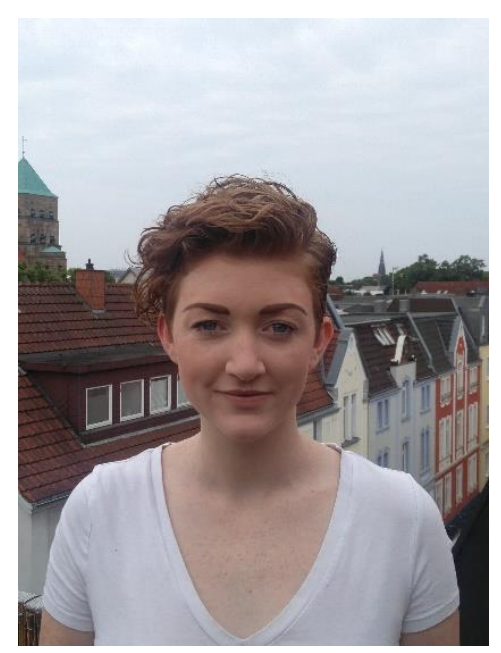

Samantha Smith studied Honours Chemistry and Mathematics as a double major at Wilfrid Laurier University where she was first exposed to research. She continued on to the University of Toronto's Department of Chemistry where she is currently working on a Ph. D.

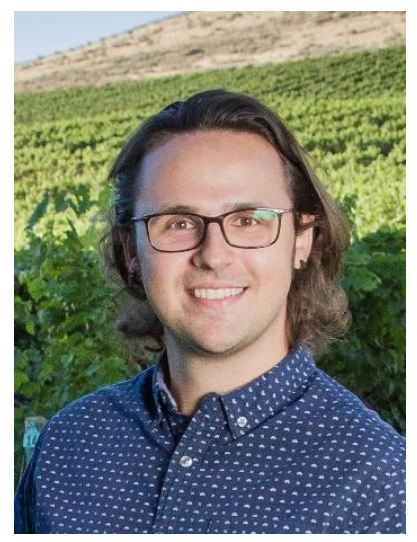

Demyan Prokopchuk was born in Saskatoon, Canada. He received his undergraduate degree from the University of Saskatchewan in 2009 and his PhD from the University of Toronto in 2015 under the supervision of Prof. Robert Morris. He is currently a postdoctoral fellow at Pacific Northwest National Laboratory in the Center for Molecular Electrocatalysis, directed by R. Morris Bullock. His current research interests include ligand design and electrochemistry for applications in small molecule activation. 


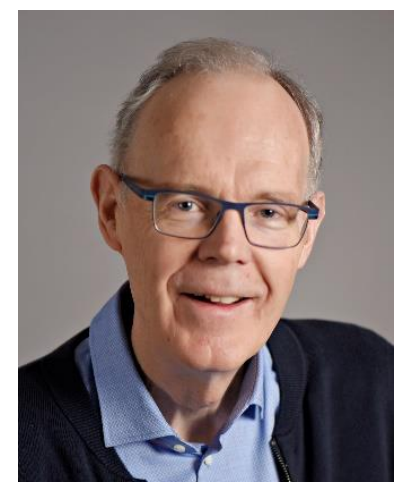

Bob Morris is a professor of chemistry at the University of Toronto. He was born in Ottawa in 1952. He received his PhD from the University of British Columbia in 1978. After postdoctoral work at the Nitrogen Fixation Laboratory, University of Sussex and the Pennsylvania State University he joined the faculty of the University of Toronto in 1980. He was appointed full Professor there in 1989 and served as Acting Chair and Chair of the Chemistry Department from 2008-2013. His research interests include inorganic, organic and catalytic chemistry with applications in the fine chemical industry. He is a Fellow of the Royal Society of Canada and of the Chemical Institute of Canada and Killam Research Fellow (2015-2017).

\section{Introduction}

The asymmetric reduction of ketones is a challenging process that is useful for many industries including pharmaceutical and fine chemical. Usually precious-metal-based catalysts are employed. ${ }^{[1]}$ The use of abundant metals such as iron for this transformation is attractive due to their lower cost and toxicity, and this has been a topic of much interest in the last decade. ${ }^{[2]}$ Recently discovered iron catalysts stand out for their high enantioselectivity and activity in the asymmetric transfer hydrogenation of ketones. ${ }^{[2 b, 2 c, 2 m}$, 3]

The activity and selectivity of our iron-based ATH catalysts (Figure 1) has steadily improved using the reduction of acetophenone by isopropanol as a test case. Our first generation ATH precatalyst $(R, R)-\mathrm{A}$ in isopropanol produced, after activation by isopropoxide, (S)-1-phenylethanol in 63\% ee (TOF 400, TON $1630 \mathrm{~h}^{-1}$ at $\left.24^{\circ} \mathrm{C}\right){ }^{[2 \mathrm{~h}, 4]}$ although this ATH system likely involves iron nanoparticles. ${ }^{[5]}$ Kinetic and mechanistic studies uncovered a high energy barrier for the activation of our more active second generation ATH precatalysts $\mathbf{B}$ that involved the reduction of one of the imine groups of the $\mathbf{B}^{[2,21,3 a]}$ Our group conducted a stereo-electronic study of complexes B that involved varying the substituents $\mathrm{R}$ of the phosphine moieties of the P-N-N-P ligand and found that only the use of aryl groups led to very active 
catalysts for the ATH of ketones. ${ }^{[6]}$ It was unclear from these studies whether some of the complexes with $\mathrm{R}=$ alkyl were inactive due to a high energy barrier to the reduction of the imine of the ligand that is needed for activation or due to a higher barrier for catalysis after imine reduction. The work described below helps to answer this question.

The design of the third generation precatalysts $\mathbf{C}$ (Figure 1 ) eliminates this activation barrier by providing the amine(imine ligand) in the structure. This dramatically increases the ATH activity of the catalyst systems in basic isopropanol when aryl groups are on the phosphine donors. The ATH of acetophenone catalyzed by $\mathbf{C 1}\left(\mathrm{R}=\mathrm{R}^{\prime}=\mathrm{Ph}\right)$ produces $(R)$-1-phenylethanol in $78 \%$ ee $(R)$ with a turnover number (TON) of 5000 and turnover frequency (TOF) $100,000 \mathrm{~h}^{-1}$ at $28^{\circ} \mathrm{C}$ while that by $\mathrm{C} 2$ gives $90 \%$ ee $(R) .{ }^{[3 b]}$ The use of other aryl ketones yielded alcohols with ee greater than $90 \%(R)$ in certain cases. ${ }^{[3 \mathrm{~b}, 7]}$ The initial work showed that a complex could be prepared with phosphorus donors with different groups on each side, namely $\mathbf{C 3}$ with $\mathrm{R}=\mathrm{Ph}, \mathrm{R}^{\prime}=4-\mathrm{MeC}_{6} \mathrm{H}_{3}$. The $\mathbf{C 3}$ system reduced acetophenone with comparable activity to C1 but with lower enantioselectivity, 70\% ee $(R) .^{\left[{ }^{[b]}\right]}$

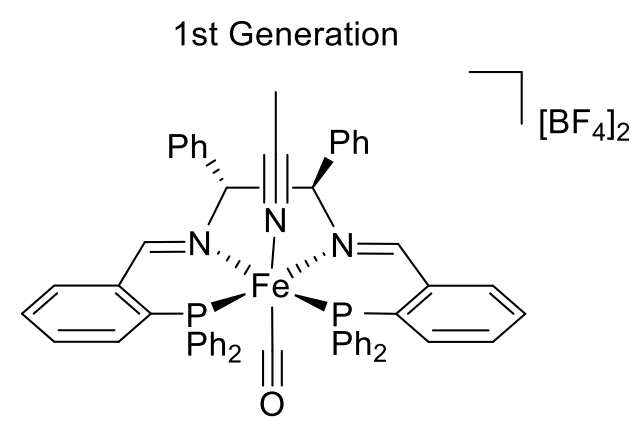

A 2nd Generation

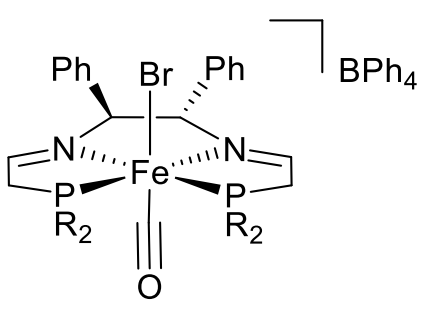

B 3rd Generation

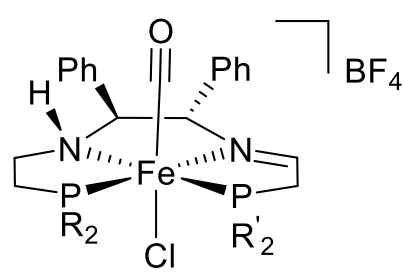

$\mathrm{C} 1 \mathrm{R}=\mathrm{R}^{\prime}=\mathrm{Ph}$

C2 $R=R^{\prime}=X y l$

C3 $R=P h, R^{\prime}=p-T o l$

Figure 1. Three generations of ATH Precatalysts.

Complex C4 with $\mathrm{R}=\mathrm{Ph}$ and $\mathrm{R}^{\prime}=\mathrm{Cy}$ (Figure 2) was found to be more stereoselective but much less active for ketone reduction than $\mathbf{C 1 - C 3 .}{ }^{[3 c]}$ For example the $\mathbf{C 4}$ system catalyzed the reduction of acetophenone to $(R)$-1-phenylethanol in $98 \%$ ee. ${ }^{[3 c]}$ The complex was assumed to have a trans configuration shown on the left of Figure 2. Recently, C4 was crystallized, and was found to adopt an unexpected cis- $\beta$ geometry (Figure 2, right). ${ }^{[8]}$ An objective of the current work is to investigate whether this cis- $\beta$ geometry is of relevance to the activity and selectivity of $\mathbf{C} 4$ relative to $\mathbf{C 1}$. 


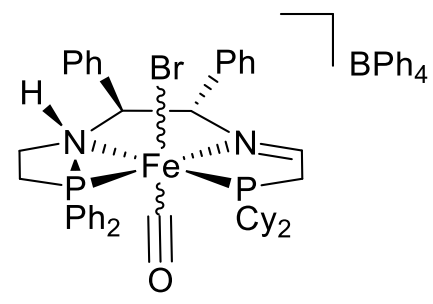

C4

Two trans diastereomers

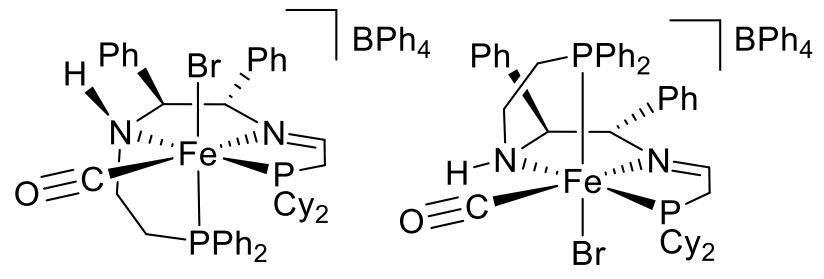

$$
\mathrm{C} 4-\boldsymbol{R}_{\mathrm{NH}}
$$

\section{C4-S $\mathrm{NH}_{\mathrm{NH}}$}

Cis- $\beta$ diastereomers in the solid state

Figure 2. Previously proposed and recently discovered geometries of $\mathbf{C 4}$ with a cis- $\beta$ stereochemistry

Herein we explore the introduction into the $\mathrm{Fe}(\mathrm{II})$ complexes trans- $\left.\mathrm{FeBr}(\mathrm{CO})\left(\mathrm{P}-\mathrm{NH}-\mathrm{N}-\mathrm{P}^{\prime}\right)\right]^{+}$of two other substituents $\mathrm{PR}_{2}^{\prime}$, the small, basic $\mathrm{PEt}_{2}$ donor and the large, less basic $\mathrm{P}(\mathrm{o}-\mathrm{Tol})_{2}$ donor while keeping $\mathrm{PR}_{2}$ group constant as $\mathrm{PPh}_{2}$. This work explores a wider range of stereochemical and electronic properties in order to better understand the relationship between the structures and the resulting activity and enantioselectivity of the catalysts for the reduction of a variety of ketone structures. Simulating the reaction progress of the reductions also sheds light on the factors that determine how quickly the ee of the product alcohol is lost after the reaction reaches equilibrium. We also investigate the properties of the active hydride-containing species.

\section{Results and Discussion}

\subsection{Preparation and characterization of complexes}

The syntheses of the new iron(II) precatalysts were carried out using routes developed in our lab previously. ${ }^{[3 b, 6 b, 9]}$ Complexes $(S, S)-\mathbf{1}$ with $R^{\prime}=E t$ and $(S, S)-2$ with $R^{\prime}=0$-Tol were synthesized using the enantiopure $(S, S)-\mathrm{PPh}_{2} \mathrm{CH}_{2} \mathrm{CH}_{2} \mathrm{NHCHPhCHPhNH}_{2}$ compound and the phosphonium dimers D1 or D2, [10] respectively, in two main steps (Scheme 1). 


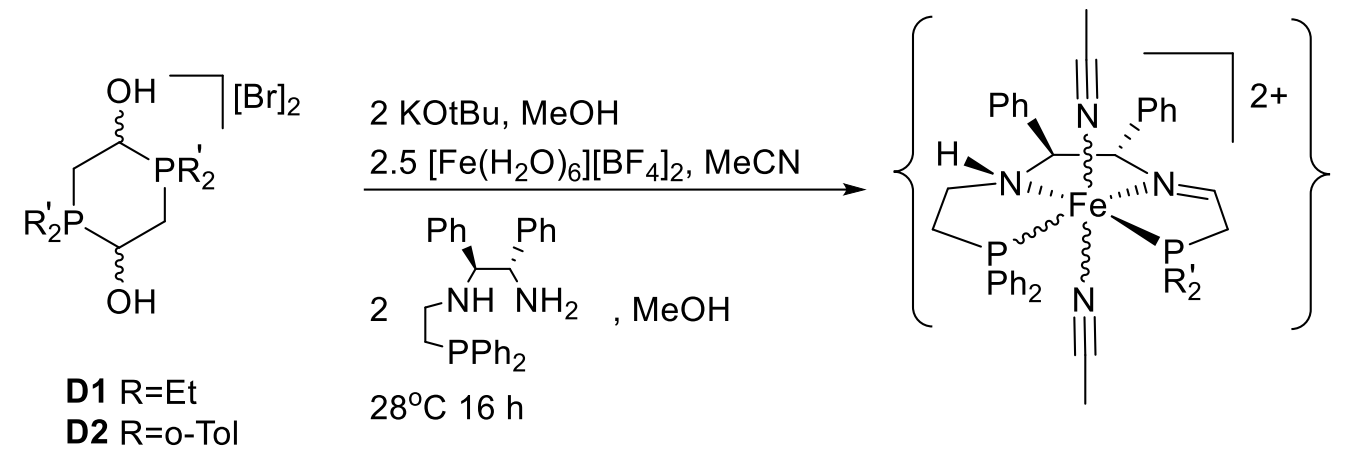

1) $4 \mathrm{KBr} / \mathrm{CO}$, Acetone
$\frac{3 \mathrm{~h}, 28^{\circ} \mathrm{C}}{\text { 2) } 1.1 \mathrm{NaBPh}_{4}, \mathrm{MeOH}}$

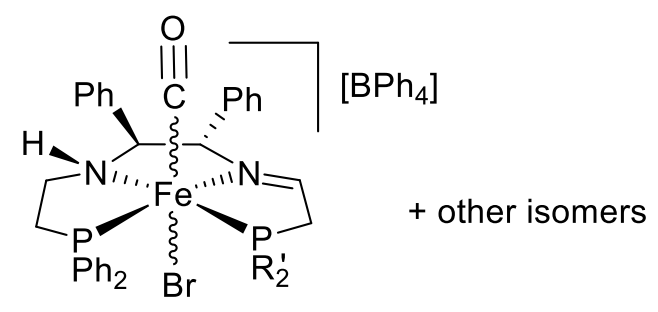

$$
\begin{aligned}
& (S, S)-1 R^{\prime}=E t \\
& (S, S)-2 R^{\prime}=0-T o l
\end{aligned}
$$

Scheme 1. The two-step synthesis of complexes $(\boldsymbol{S}, \mathbf{S})-\mathbf{1}$ and $(\mathbf{S}, \mathbf{S})-\mathbf{2}$

First an intermediate complex is made in situ by the reaction of the appropriate phosphonium dimer with base, $\mathrm{Fe}(\mathrm{II})$, and an enantiopure (S,S)-PNN ligand in acetonitrile. On the basis of previous work ${ }^{[3 \mathrm{~b}, 6 \mathrm{~b}, 9]}$ this is assumed to be a trans-bis(acetonitrile) complex. This is then treated with $1 \mathrm{~atm} C \mathrm{CO}$ and excess $\mathrm{KBr}$. The complexes $(\mathbf{S}, \mathbf{S})-\mathbf{1}$ and $(\boldsymbol{S}, \boldsymbol{S})-\mathbf{2}$ were precipitated as the $\mathrm{BPh}_{4}{ }^{-}$salts in 35 and-56\% yield, respectively, with respect to the starting $(S, S)-\mathrm{P}-\mathrm{NH}-\mathrm{NH}_{2}$ compound. Complex $(S, S)-1$ was produced as a mixture of two trans diastereomers with structures $\mathbf{E}$ and $\mathbf{E}^{\prime}$ (Figure 3) with NMR properties very similar to $\mathbf{C} \mathbf{1}$ and $\mathbf{C} \mathbf{4}$ in addition to a small fraction (17\%) of two other isomers with poorly resolved doublet resonances at 62,59 and 55 ppm (Table 1). These minor isomers might have the cis- $\beta$ geometries shown in Figure 2 but this could not be definitively established. Similarly $(\boldsymbol{S}, \boldsymbol{S})-\mathbf{2}$ appears to have two trans isomers (75\% of mixture) and at least one additional isomer at 58 and $63 \mathrm{ppm}$. Each diastereomer has a characteristic set of doublets in the ${ }^{31} \mathrm{P}\left\{{ }^{1} \mathrm{H}\right\}$ NMR spectrum (see Table 1 ). 


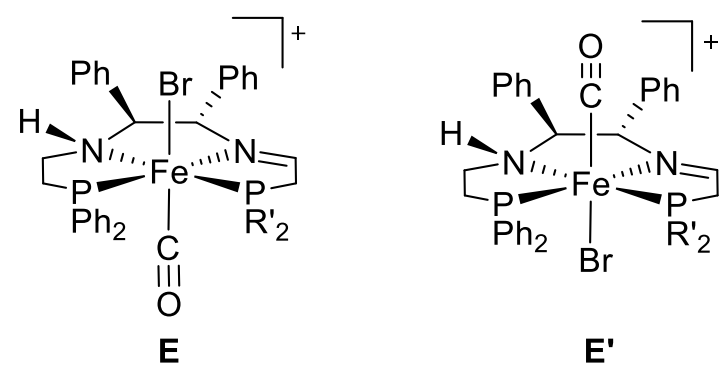

Figure 3. Geometries of the trans tetradentate complexes.

Table 1. ${ }^{31} \mathrm{P}\left\{{ }^{1} \mathrm{H}\right\}$ NMR resonances of the trans isomers of $\mathbf{C 1}, \mathbf{C 4},(\mathbf{S}, \mathbf{S})-\mathbf{1}$ and $(\mathbf{S}, \mathbf{S})-\mathbf{2}$ with structures $\mathbf{E}$ or $\mathbf{E}^{\prime}$ of Figure 3.

\begin{tabular}{|c|c|c|c|c|c|c|}
\hline Complex & $\mathrm{PR}_{2}{ }_{2}$ & $\begin{array}{l}\text { isomer } \\
\text { fraction (\%) }\end{array}$ & $\mathrm{PR}_{2}{ }_{2}(\mathrm{ppm})$ & $\mathrm{PPh}_{2}$ (ppm) & ${ }^{2} J_{\mathrm{PP}}(\mathrm{Hz})$ & Struct. \\
\hline C1 & $\mathrm{PPh}_{2}$ & 100 & 62.6 & 58.0 & 40 & $E$ \\
\hline C4 & $\mathrm{PCy}_{2}$ & 75 & 78.4 & 47.3 & 33 & $E$ or $E^{\prime}$ \\
\hline$(S, S)-1-1$ & $\mathrm{PEt}_{2}$ & 38 & 65.1 & 59.3 & 38 & $E^{\prime}$ or $E$ \\
\hline$(S, S)-1-2$ & $\mathrm{PEt}_{2}$ & 45 & 67.1 & 53.7 & 36 & $E^{\prime}$ or $E^{\prime}$ \\
\hline$(S, S)-2-1$ & $\mathrm{Po}-\mathrm{Tol}_{2}$ & 41 & 61.6 & 55.4 & 41 & $E^{\prime}$ or $E^{\prime}$ \\
\hline$(S, S)-2-2$ & $\mathrm{Po}^{-} \mathrm{Tol}_{2}$ & 34 & 63.7 & 54.1 & 39 & $E^{\prime}$ or $E$ \\
\hline
\end{tabular}

One relatively broad $\left(w_{1 / 2} 20 \mathrm{~cm}^{-1}\right) \mathrm{CO}$ absorption $\left(\mathrm{v}_{\mathrm{CO}}\right)$ is observed in the IR spectrum for the iron(II) complexes as mixtures of isomers as listed in Table 2 for the two complexes as well as the complexes $\mathbf{C 1}$ and C4 for comparison (Table 2). Only one peak maximum representing an averaged electronic property of the various isomers present is reported because separate peak maxima were not resolved. There is no clear trend in the $\mathrm{v}_{\mathrm{CO}}$ values. The steric parameters of the $\mathrm{PR}_{2}{ }_{2} \mathrm{CH}_{2}$ - group can be expressed in terms of Tolman's cone angles $\theta$ of the corresponding $\mathrm{PR}_{2}{ }_{2} \mathrm{Et}$ ligands as also listed in Table 2. ${ }^{[1]}$

Table 2. Stereoelectronic effects represented by Tolman's cone angle and IR wavenumbers of the CO ligand.

\begin{tabular}{|l|l|l|l|}
\hline Complex & $\mathrm{PR}_{2}$ & IR $\mathrm{v}_{\mathrm{co}}\left(\mathrm{cm}^{-1}\right)$ & $\begin{array}{l}\text { Tolman's Cone Angle } \\
(\theta)^{[\mathrm{a}]}\end{array}$ \\
\hline $\mathbf{( S , S ) - 1}$ & $\mathrm{PEt}_{2}$ & 1956 & 132 \\
\hline $\mathbf{C 1}-\mathbf{B r}^{[\mathrm{b}]}$ & $\mathrm{PPh}_{2}$ & $1979^{[\mathrm{b}]}$ & 141 \\
\hline
\end{tabular}




\begin{tabular}{|l|l|l|l|}
\hline $\mathbf{C 4}$ & $\mathrm{PCY}_{2}$ & 1960 & 157 \\
\hline$(\mathbf{S}, \mathbf{S})-\mathbf{2}$ & $\mathrm{Po}^{-\mathrm{Tol}_{2}}$ & 1963 & 173 \\
\hline
\end{tabular}

[a] Cone angle of $\mathrm{R}_{2} \mathrm{PEt}$ representing the $\mathrm{PR}_{2}{ }_{2} \mathrm{CH}_{2}$ fragment of the complex. [b] Like $\mathrm{C} 1$ from Figure 1 , but synthesized with a bromo instead of a chloro ligand for better comparison.

\subsection{Analysis of the structures of the isomers in solution.}

Complex $\mathbf{C 3}$ has been characterized crystallographically in the trans configuration with the NH locked anti with respect to the hydrogen of the adjacent $\mathrm{CHPh}$ group with $(S)$ chirality as shown in Figure 1. All 43 racemic or enantiopure dpen metal derivatives in the Cambridge Crystallographic Databank have the NH and $\mathrm{CH}$ locked anti (see the Supporting information). The similarity of the NMR spectra of complexes C1 to $\mathbf{C} 3$ suggest that the major isomers all have this trans structure as do a wide variety of more symmetrical trans- $\left[\mathrm{Fe}(\mathrm{CO}) \mathrm{Br}\left(\mathrm{PR}_{2}-\mathrm{N}-\mathrm{N}-\mathrm{PR}_{2}\right)\right]^{+}$complexes reported by our group with a wide range of substituents (Et, Cy, aryl). ${ }^{[21,6]}$

The cis- $\beta$ structure of $\mathbf{C} \mathbf{4}$ in the solid state introduces another possibility for structural assignments and it has been observed in related iron and ruthenium complexes with tetradentate phosphorus and nitrogen ligands ${ }^{[2 b, 12]}$. However in solution the main isomer of $\mathbf{C 4}$ (75\%) has the trans configuration with either the $\mathrm{NH}$ next to the $\mathrm{CO}$ ligand (structure $\mathbf{E}$ ) or the $\mathrm{Br}$ ligand $\left(\mathbf{E}^{\prime}\right)$ as in Figure 3. This is determined by assigning all of the protons around the backbone of the ligand using 2D NMR experiments and spin simulations and demonstrating the similarity to the proton spectra of trans complex $\mathbf{C 1}$ (see the Supporting Information). In particular the ${ }^{3}{ }_{H H}$ coupling constants of the $\mathrm{PPh}_{2} \mathrm{CH}_{2} \mathrm{CH}_{2} \mathrm{NH}$ part of the ligand backbone should be sensitive to the differences in dihedral angles between the trans and the cis- $\beta$ configurations where this part of the tetradentate ligand that folds away from the PNN plane. The simulated ${ }^{3} J_{H H}$ couplings are similar for the $\mathbf{C} \mathbf{1},(\mathbf{S}, \mathbf{S})$-1 and $\mathbf{C} 4$ isomers in the assigned trans-configuration. The large ${ }^{3}{ }_{H H}$ coupling $(12-14 \mathrm{~Hz})$ indicative of anti-vicinal $\mathrm{CH}$ groups in the trans structure is present in the ${ }^{1} \mathrm{H}$ spectra of all of these compounds. The minor isomer of $\mathbf{C} 4$ with ${ }^{31} \mathrm{P}\left\{{ }^{1} \mathrm{H}\right\}$ NMR signals at 76.0 and $75.5 \mathrm{ppm}$ probably has a cis- $\beta-\mathbf{C} 4$ structure, but it is in too low a concentration to provide definitive proton assignments. In another sample prepared for HMBC NMR analysis, another trans-isomer, (E or $\mathbf{E}^{\prime}$ ) with ${ }^{31} \mathrm{P}\left\{{ }^{1} \mathrm{H}\right\} \mathrm{NMR}$ resonances at $73.0\left(\mathrm{~d},{ }^{2} J_{\mathrm{pP}} 35 \mathrm{~Hz}\right)$ and $51.8 \mathrm{ppm}\left(\mathrm{d},{ }^{2} J_{\mathrm{PP}} 35 \mathrm{~Hz}\right)$ were also observed. Thus the $\mathrm{PPh}_{2} \mathrm{CH}_{2} \mathrm{CH}_{2}$ arm of $\mathrm{C} 4$ is mobile and allows a switch that is slow on the NMR timescale between the structures shown in Figure 2. 
A ${ }^{1} \mathrm{H}-{ }^{31} \mathrm{P}$ HMBC experiment and spin simulations were used to assign the structures of the trans isomers of complex (S,S)-1, although it was not possible to distinguish which of the two diastereomers was $\mathbf{E}$ vs $\mathbf{E}^{\prime}$ of Figure $3\left(\mathrm{PR}_{2}{ }_{2}=\mathrm{PEt}_{2}\right)$. Table 1 lists the ${ }^{31} \mathrm{P}\left\{{ }^{1} \mathrm{H}\right\}$ chemical shifts and coupling constants of these isomers. Obtaining good NMR spectra for $(S, S)-2$ was challenging but its ${ }^{31} \mathrm{P}\left\{{ }^{1} \mathrm{H}\right\}$ NMR spectra appear to be similar to those of $(\mathbf{S}, \mathbf{S})-\mathbf{1}$.

\subsection{Catalytic results}

\subsubsection{Acetophenone reduction and reaction progress modelling}

The ATH of acetophenone (Figure 4) was carefully examined for catalysts $(\boldsymbol{S}, \mathbf{S})-\mathbf{1},(\boldsymbol{S}, \mathbf{S})-\mathbf{2}$ as well as $\mathbf{C 1}$ and $\mathbf{C 4}$ for comparison under a range of conditions (Figure 4).

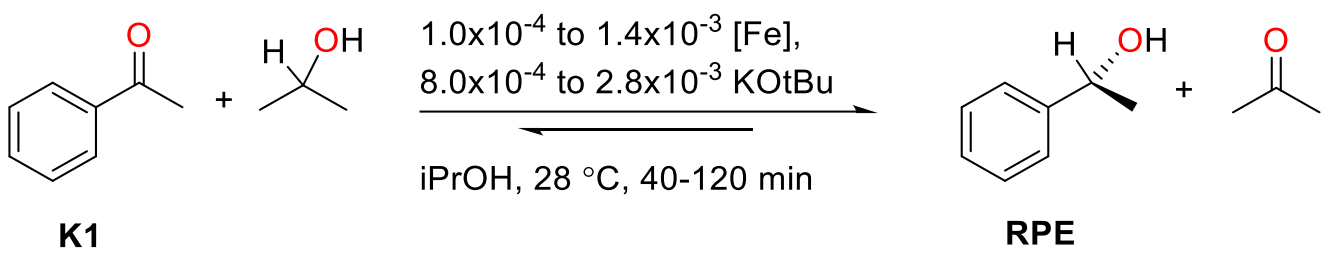

Figure 4. Conditions for the ATH of acetophenone.

Initial catalytic reactions were done with acetophenone (K1) with a precatalyst/KOtBu/substrate ratio (C/B/S) of $1 / 2 / 500$ with [K1] $0.70 \mathrm{M}$ in iPrOH at $28^{\circ} \mathrm{C}$. Figure 5 shows that complexes $(S, S)$ 1 with the small $\mathrm{PEt}_{2}$ group reduces acetophenone to 1-phenylethanol to the equilibrium point of $86 \%$ conversion with a high initial TOF of approx. $12 \mathrm{~s}^{-1}$ as determined by reaction profile fitting (see below). The conversion can be increased to over $90 \%$ by using a lower concentration of ketone (e.g. 0.1 M). The complexes $\mathrm{C} 4$ and $(\mathbf{S}, \mathbf{S})-2$ with bulkier $\mathrm{PCy}_{2}$ and $\mathrm{Po}^{-\mathrm{Tol}_{2}}$ groups have lower initial TOF and lose activity before reaching equilibrium. On the other hand the last two complexes maintain a high ee in the product while $(\boldsymbol{S}, \boldsymbol{S})-\mathbf{1}$ has significant losses in ee over time because of its poor selectivity. There is less ee degradation over the course of the reaction when using a lower catalyst loading ${ }^{[3 \mathrm{~b}]}$ with the $\mathrm{C} / \mathrm{B} / \mathrm{S}$ ratio $1 / 8 / 6121$ with [ketone] $0.63 \mathrm{M}$ (Figure 6). C4 and (S,S)-2 were again slower at reducing acetophenone; however the ee for $\mathbf{C 4}$ was much higher $(98 \% R)$ at maximum conversion. The reaction slows after $40 \%$ conversion and attains only $80 \%$ of the possible conversion at $120 \mathrm{~min}$. This is also true for $(\mathbf{S}, \mathbf{S})-\mathbf{2}$. $(\mathbf{S}, \mathbf{S})-\mathbf{2}$ produces the alcohol in $89 \%$ ee $(R)$ after 120 minutes. $(S, S)-1$ has the higher activity to a maximum conversion 
of $87 \%$ after $50 \mathrm{~min}$, but also a lower ee of $85 \%$. For comparison $\mathrm{C} 1$ with $\mathrm{PPh}_{2}$ groups produces 1-phenethanol at $82 \%$ ee at the time of maximum conversion. ${ }^{[3 \mathrm{~b}]}$
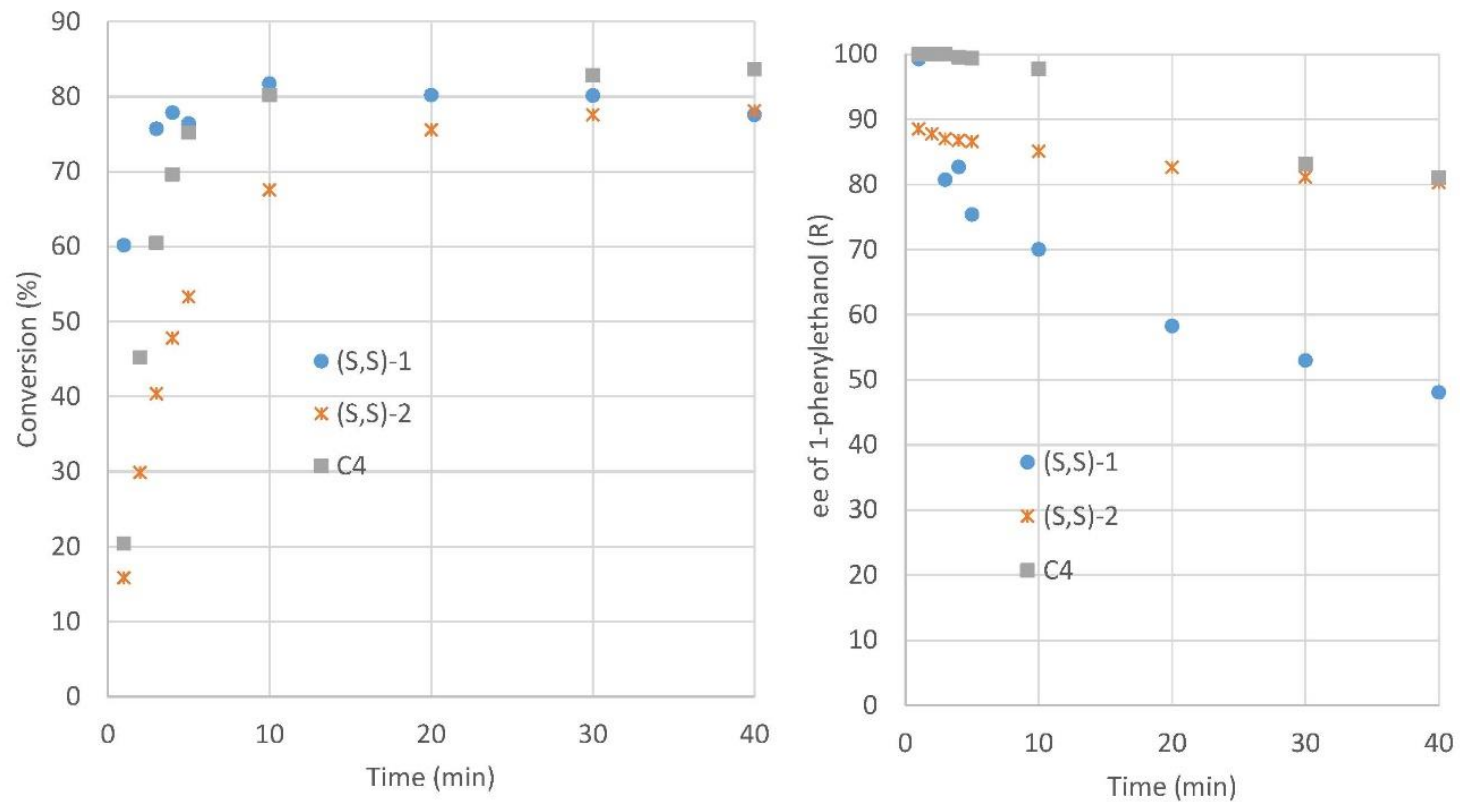

Figure 5. ATH of acetophenone (K1) with $(S, S)-1,(S, S)-2$ and C4. Reaction conditions: $28^{\circ} \mathrm{C}, 1.4 \times 10^{-3} \mathrm{M}$ precatalyst, $2.8 \times 10^{-3} \mathrm{M} \mathrm{KOtBu}, 0.70 \mathrm{M}$ acetophenone, $6 \mathrm{~mL}$ iPrOH, C/B/S=1/2/500; \% conversion and \% ee determined by chiral GC. 

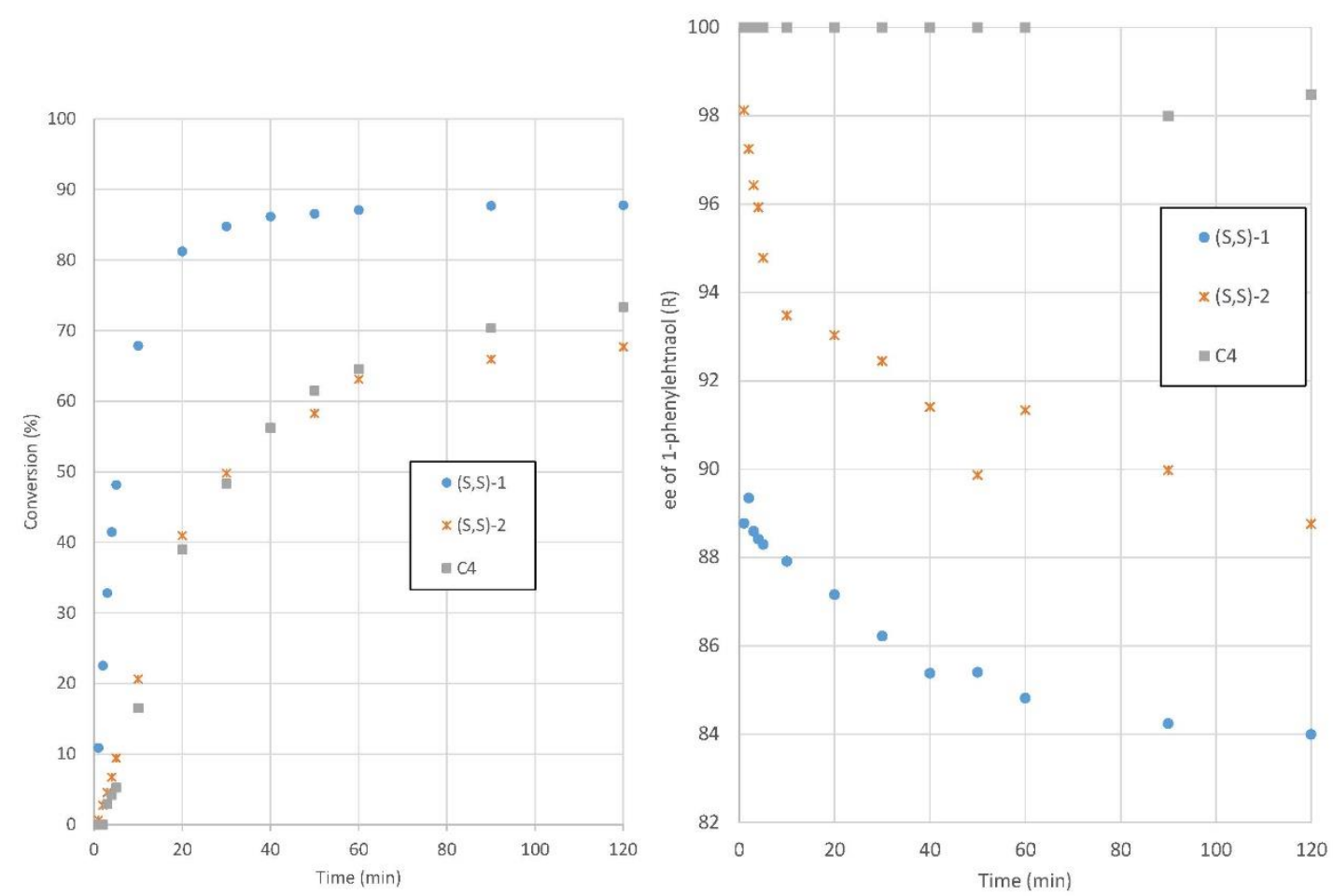

Figure 6. ATH of $\mathrm{K} 1$ with $(S, S)-1,(S, S)-2$ and $\mathrm{C} 4$. Reaction conditions: $28^{\circ} \mathrm{C}, 1.0 \times 10^{-4} \mathrm{M}$ precatalyst, $8 \times 10^{-4}$ $\mathrm{M} \mathrm{KOtBu}, 0.63 \mathrm{M}$ acetophenone, $6 \mathrm{~mL}$ iPrOH, C/B/S=1/8/6121; \% conversion and \% ee determined by chiral GC.

The reaction progress and changes in ee for the ATH of acetophenone (AP) were semiquantitatively fit for the first time to a simple kinetic model ${ }^{[13]}$ for a reversible equilibrium using the program Dynafit ${ }^{[14]}$ (Figures 7-10). The progress of the reactions catalyzed by $(\mathbf{S}, \mathbf{S})-\mathbf{1},(\mathbf{S}, \mathbf{S})-\mathbf{2}$, C1 and C4 were fit to only three parameters: two rate constants $k_{R}$ and $k_{s}$ for pseudo first order reactions producing $(R)$ - and $(S)$-1-phenylethanol (abbreviated RPE and SPE, respectively) and one equilibrium constant $\left(K_{r a c}=2 k_{R} / k_{-R}=2 k_{s} / k_{-s}\right)$ for the reversible reaction of acetophenone with isopropanol to give 1-phenylethanol and acetone. The same $K_{\mathrm{rac}}=12$ applies to all of the reactions of Figures 5 and 6 . From the rate constants the intrinsic enantioselectivity of the catalyst is obtained $\left(S=k_{R} / k_{S}\right) .{ }^{[13]}$ The value of $S$ determines the initial ee of the system. The initial TOF is given by $\left(k_{R}+k_{S}\right)[A P] /[C a t]$ and the initial e.e. is given by $\left(k_{R}-k_{S}\right) /\left(k_{R}+k_{S}\right)$.

This simple approach makes the following assumptions: 
1. The forward reaction to produce RPE is first order in AP with a steady-state catalyst concentration included in the rate constant $k_{R}$.

2. The forward reaction to produce SPE is first order in AP with a steady-state catalyst concentration included in the rate constant $\mathrm{ks}_{\mathrm{s}}$.

3. At equilibrium, the concentrations of RPE and SPE will be equal ( 0 ee) and the equilibrium constant will be $\mathrm{K}_{\mathrm{rac}}=2 \mathrm{~K}_{\mathrm{R}}=2 \mathrm{~K}_{\mathrm{S}}=12.0$ for the conditions described here.

4. The backward reaction to produce AP from RPE is first order in RPE with a steady-state catalyst concentration included in the rate constant $k_{-R}=k_{R} / K_{R}=2 k_{R} / K_{\text {rac }}$.

5. The backward reaction to produce AP from SPE is first order in SPE with a steady-state catalyst concentration included in the rate constant $k_{-s}=k_{s} / K_{s}=2 k_{s} / K_{\text {rac. }}$.

Assumptions 4 and 5 explain why the ee of the product degrades with the progress of the reaction if $\mathrm{ks}_{\mathrm{s}}$ is non-negligible.

The model was validated using data from the well-defined catalyst C1 (Figure 7). The parameters obtained are listed in Table 3. Catalyst $\mathbf{C 1}$ with two moderately sized $\mathrm{PPh}_{2}$ groups has an extremely high TOF of $250 \mathrm{~s}^{-1}$ as documented elsewhere ${ }^{[3 \mathrm{~b}]}$ and an inherent selectivity $S$ of 12 , resulting in a starting ee of $85 \%$ which degrades to $78 \%$ at maximum conversion at $120 \mathrm{~s}$. The experimental ee have larger uncertainties early in the reaction because of the low concentration of the $(S)$-1-phenylethanol in the sample but the model fits the general trends in the ee. 

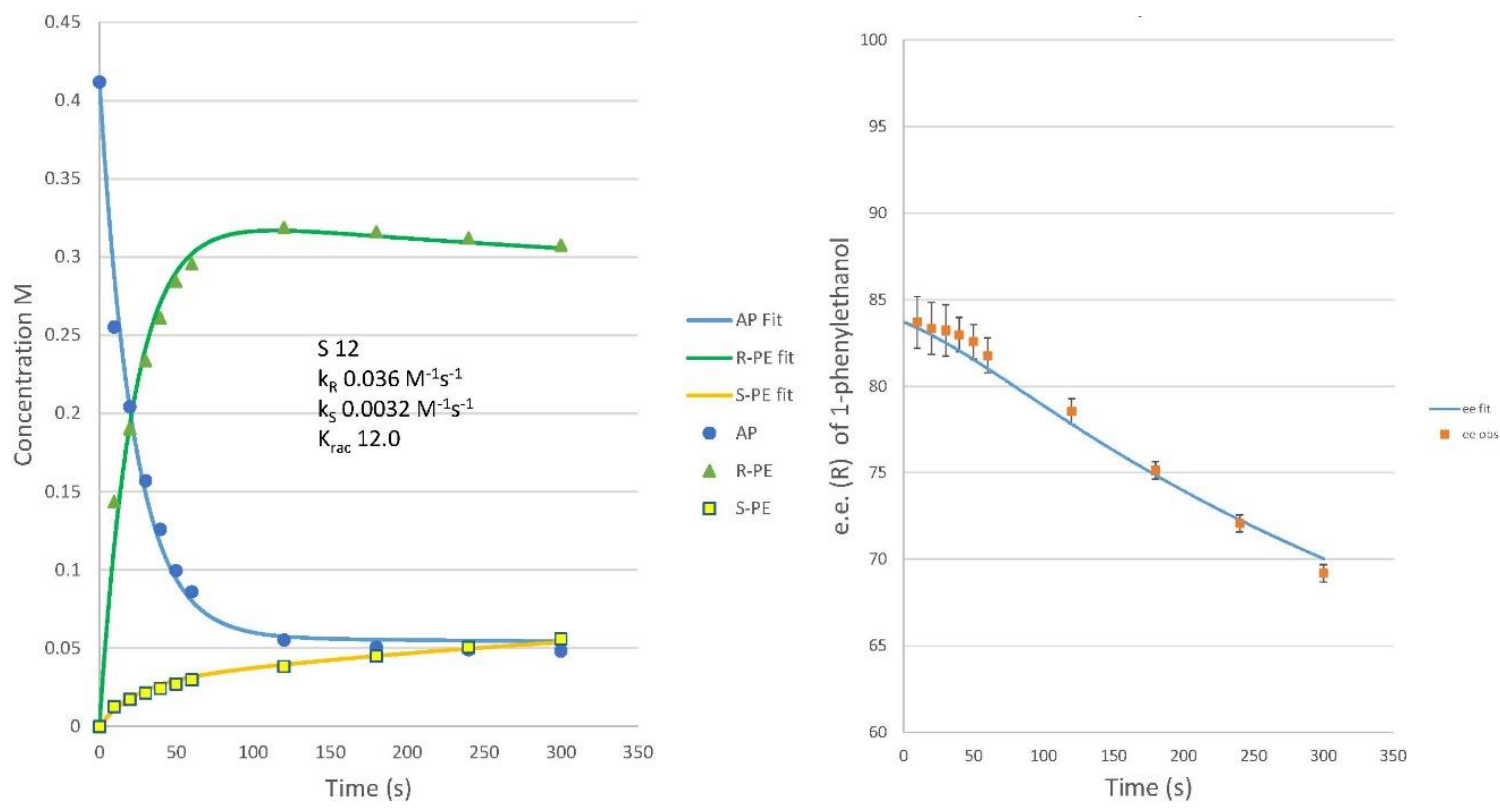

Figure 7. (Left) Kinetic fit to the changes in concentration in the ATH of acetophenone catalyzed by C1. (Right) Fit to the changes in ee for the reaction.

Table 3. Results of fitting the reaction profiles. ${ }^{1}$

\begin{tabular}{|c|c|c|c|c|c|c|c|}
\hline Catalyst & $k_{R}\left(s^{-1}\right)$ & $k_{s}\left(s^{-1}\right)$ & $\operatorname{TOF}\left(\mathrm{s}^{-1}\right)$ & $s$ & $\begin{array}{l}\text { ee } \\
\text { initial } \\
(R \%)\end{array}$ & $\begin{array}{l}\text { ee (at } \\
\text { time, min) }\end{array}$ & TON \\
\hline $\mathrm{C1}^{2}$ & $3.6 e-2$ & $3.2 \mathrm{e}-3$ & 235 & 12 & 85 & $78(2)$ & 5900 \\
\hline$(S, S)-1$ & $2.1 \mathrm{e}-3$ & $1.1 \mathrm{e}-4$ & 14 & 19 & 90 & $80(40)$ & 5000 \\
\hline$(S, S)-2^{3}$ & $4.0 \mathrm{e}-4$ & $2.0 e-6$ & 6 & 200 & 99 & $93(4)$ & 500 \\
\hline$(S, S)-2^{3}$ & $4.1 \mathrm{e}-4$ & $1.7 e-5$ & 3 & 28 & 92 & $90(90)$ & 3700 \\
\hline C4 & $4.5 e-4$ & $1 e-6$ & 3 & 510 & 99.6 & $98.5(120)$ & 4400 \\
\hline
\end{tabular}

${ }^{1}\left[\mathrm{FeBr}(\mathrm{CO})\left(\mathrm{PR}_{2} \mathrm{CH}_{2} \mathrm{CHNCHPhCPhNHCH}{ }_{2} \mathrm{CH}_{2} \mathrm{PPh}_{2}\right)\right] \mathrm{BPh}_{4}$ : [Cat]= 1.05e-4 M, [KOtBu]=8e-4 M, $[A P]=0.63 \mathrm{M}, 28^{\circ} \mathrm{C}$ in isopropanol, $\mathrm{K}_{\mathrm{rac}}=12.0 .^{2}\left[\mathrm{FeCl}(\mathrm{CO})\left(\mathrm{PPh}_{2} \mathrm{CH}_{2} \mathrm{CHN}-\right.\right.$

$\left.\left.\mathrm{CHPhCPhNHCH}_{2} \mathrm{CH}_{2} \mathrm{PPh}_{2}\right)\right] \mathrm{BF}_{4}$ : [Cat] $=6.8 \mathrm{e}-5 \mathrm{M}$, [KOtBu] $=5.4 \mathrm{e}-4 \mathrm{M},[\mathrm{AP}]=0.41 \mathrm{M}, 28^{\circ} \mathrm{C}$ in 
isopropanol, $\mathrm{K}_{\mathrm{rac}}=12.0{ }^{3}$ There is a change in catalyst structure over the course of the first 250 seconds.

The fits to the reaction progress for (S,S)-1 (Figure 8) show that this system is slower (TOF $14 \mathrm{~s}^{-1}$ ) than $\mathbf{C 1}$ but more enantioselective (S 20). Thus there are dramatic effects in changing one $\mathrm{PPh}_{2}$ (on C1) with one $\mathrm{PEt}_{2}$ group.
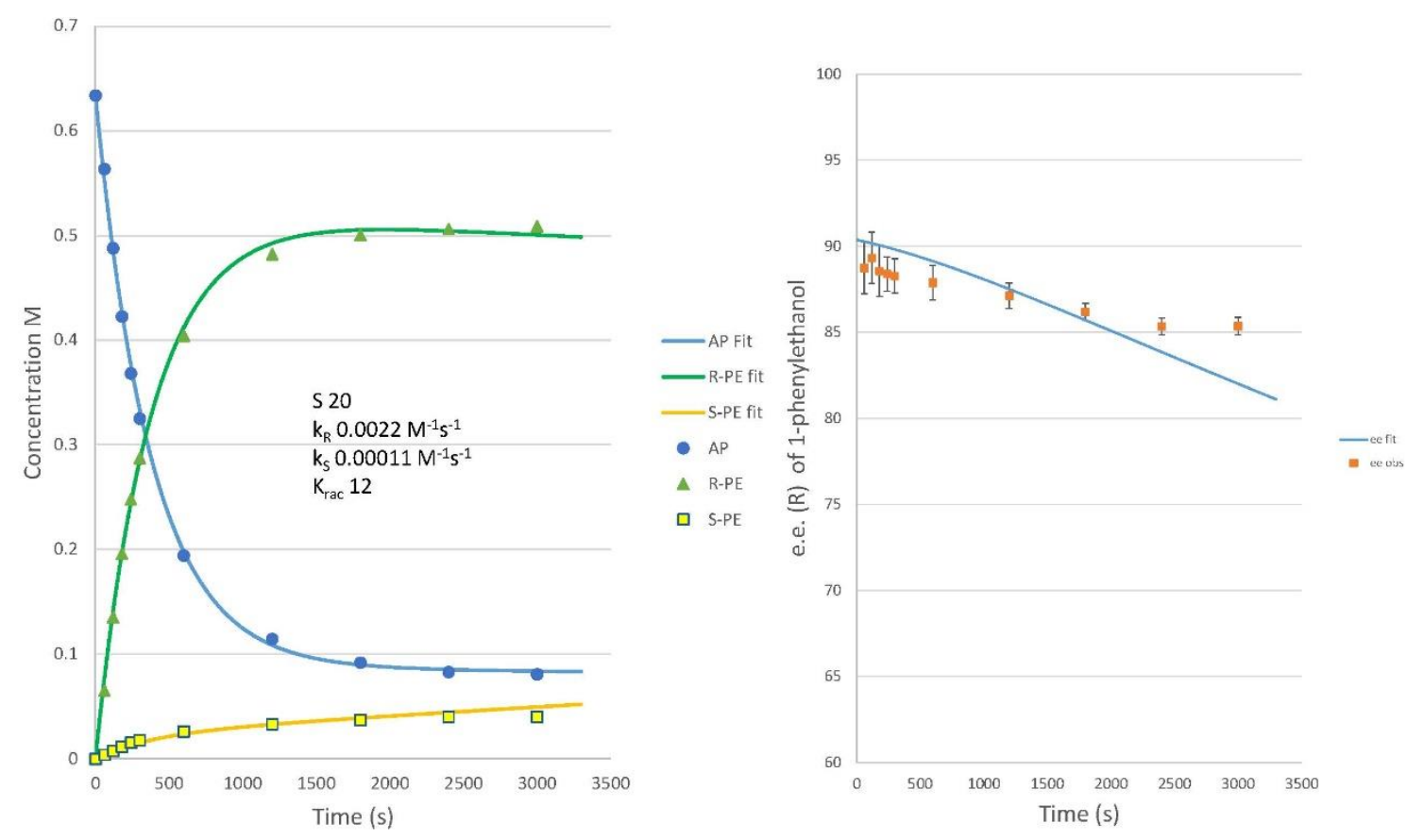

Figure 8. Simulation of the reaction progress of $(S, S)-1$ of Figure 6.

(S,S)-2 and C4 do not fit the simple model (Figures 9 and 10). The reactions slow more than expected with conversion and this indicates that the catalyst concentration is being reduced over the course of the catalytic run. This may be due to inhibition due to binding of the product alcohol. It is interesting that for $(S, S)-2$, the ee drops rapidly from a high value of $99 \% R$ over the course of the first 250 seconds. This is modelled by a more enantioselective catalyst $(S=200)$ being completely replaced by a less selective one (with $S=28$ ) over this time period. This is likely to be caused by the dissociation and repositioning of the bulky $\mathrm{CH}_{2} \mathrm{CH}_{2} \mathrm{P}(o-\mathrm{Tol})_{2}$ arm of the ligand in an as-of-yet, undefined way. Complex $\mathrm{C} 4$ with the slightly smaller $\mathrm{PCy}_{2}$ group is more stable and produces the alcohol in exceptionally high ee up to maximum conversion. 

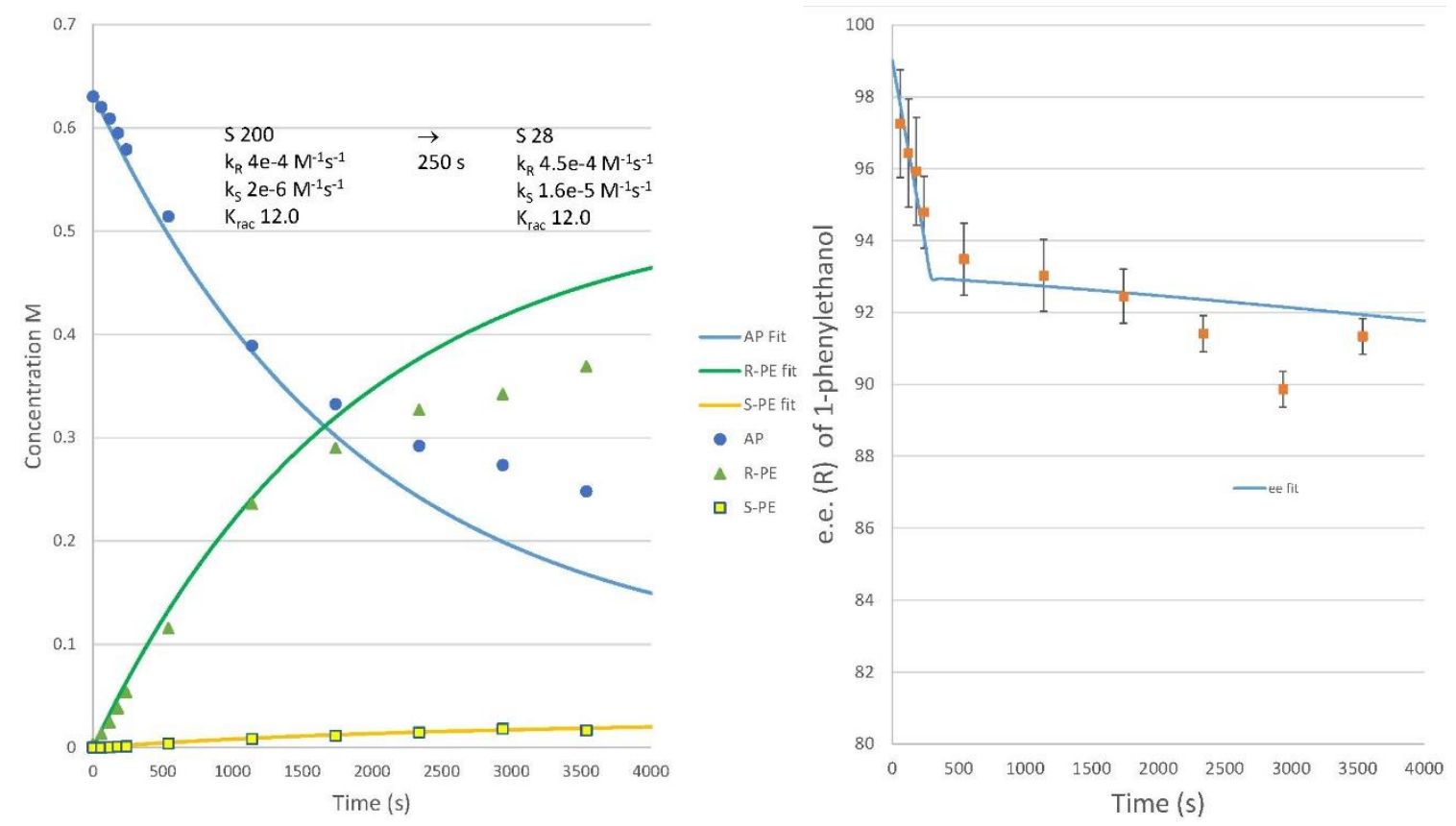

Figure 9. Simulation of the reaction progress of $(S, S)-2$ of Figure 6 . The catalyst is converted over $250 \mathrm{~s}$ from one that is very enantioselective $(S=200)$ to one that is less $(S=28)$.
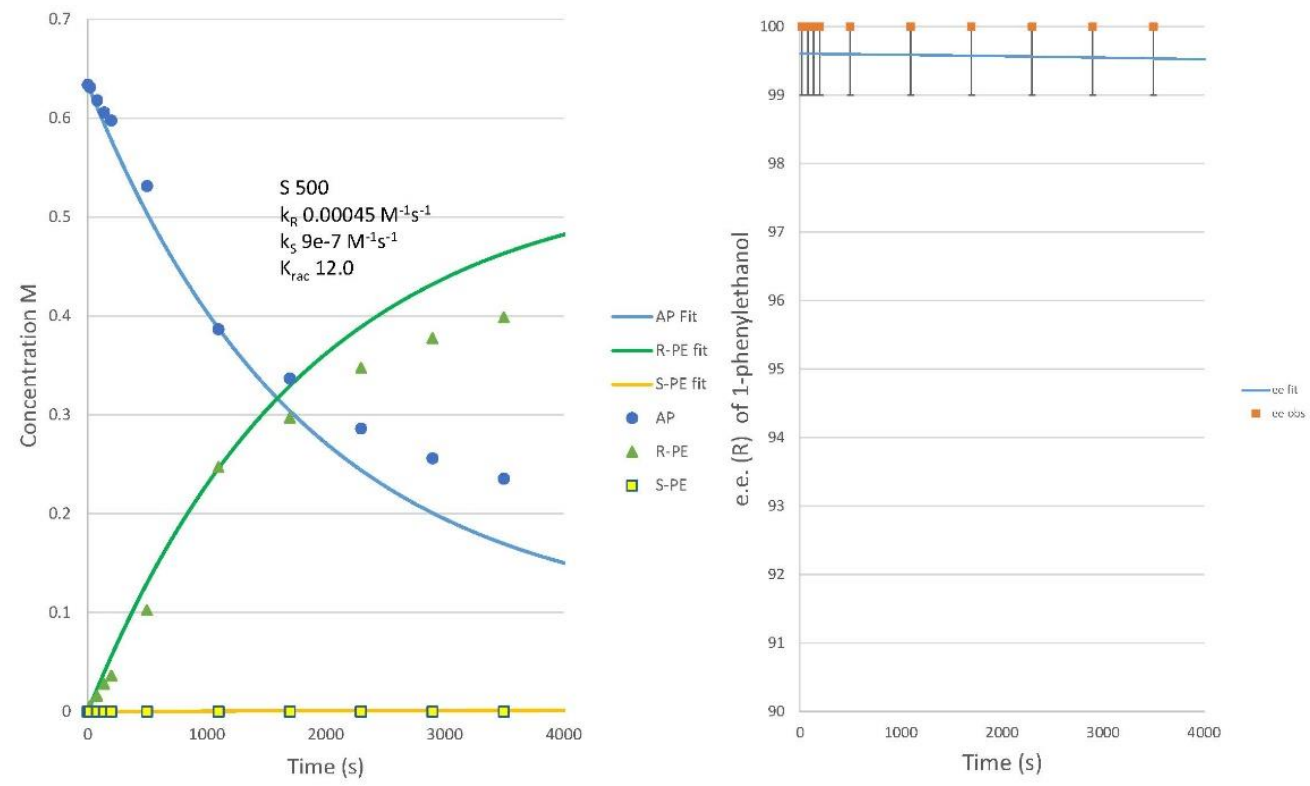

Figure 10. Simulation of the reaction progress of C4 of Figure 6.

The TOF and $S$ values obtained from fitting the reaction profiles are plotted as a function of the cone angle of the $\mathrm{PR}_{2}{ }_{2} \mathrm{CH}_{2}$ group in the complex Figures 11 and 12 . Variations in the electronic environment as reflected in the $v(\mathrm{CO})$ of the complexes are too small to show a meaningful 
variation with TOF or $S$. As far as activity (TOF) is concerned there is a region in the plot around the size of the $\mathrm{PPh}_{2}$ group where the TOF is high (Figure 11). The $\mathrm{PPh}_{2}$ complex $\mathbf{C} 1$ is an order of magnitude more active than the $\mathrm{PEt}_{2}$ and $\mathrm{PiPr}_{2}$ complexes. The use of bulky $\mathrm{PCy}_{2}$ and $\mathrm{Po}^{-\mathrm{Tol}_{2}}$ groups result in lower TOF. This is consistent with our earlier stereoelectronic study of precatalysts $\mathbf{B}$ (Figure 1 ) indicating that both studies reflect the actual activity of the catalyst and not the activation of the precatalyst; an induction period was observed for catalysis with B because an imine in $\mathbf{B}$ has to be reduced to an amine ${ }^{[3 a]}$ while no induction period is observed for the catalyst systems discussed here.

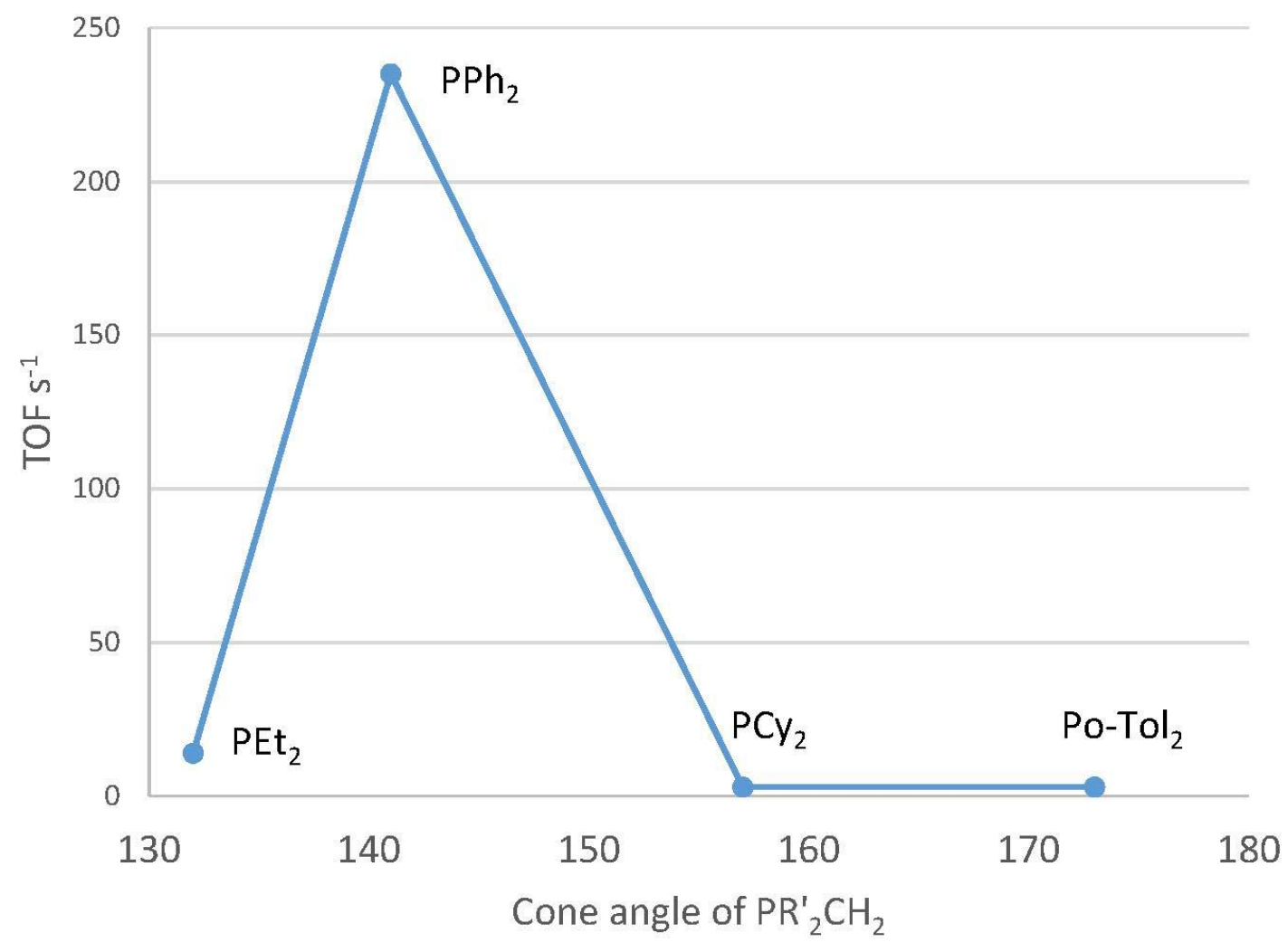

Figure 11. TOF $\left(\mathrm{s}^{-1}\right)$ for the ATH of acetophenone catalyzed by complexes as a function of cone angle $\left(^{\circ}\right)$ of the $\mathrm{PR}^{\prime}{ }_{2}$ group (see Tables 2 and 3). Reaction conditions are as in Figure 6.

However as far as the intrinsic enantioselectivity $S$ is concerned, it rises from less than 20 for the smaller substituents ( $\mathrm{PEt}_{2}, \mathrm{PPh}_{2}$ ) to a maximum of 510 for bulky $\mathrm{PCy}_{2}(98 \%$ ee) and then falls again to 200 and then to 28 for the very bulky Po-Tol 2 as discussed above (Figure 12). 


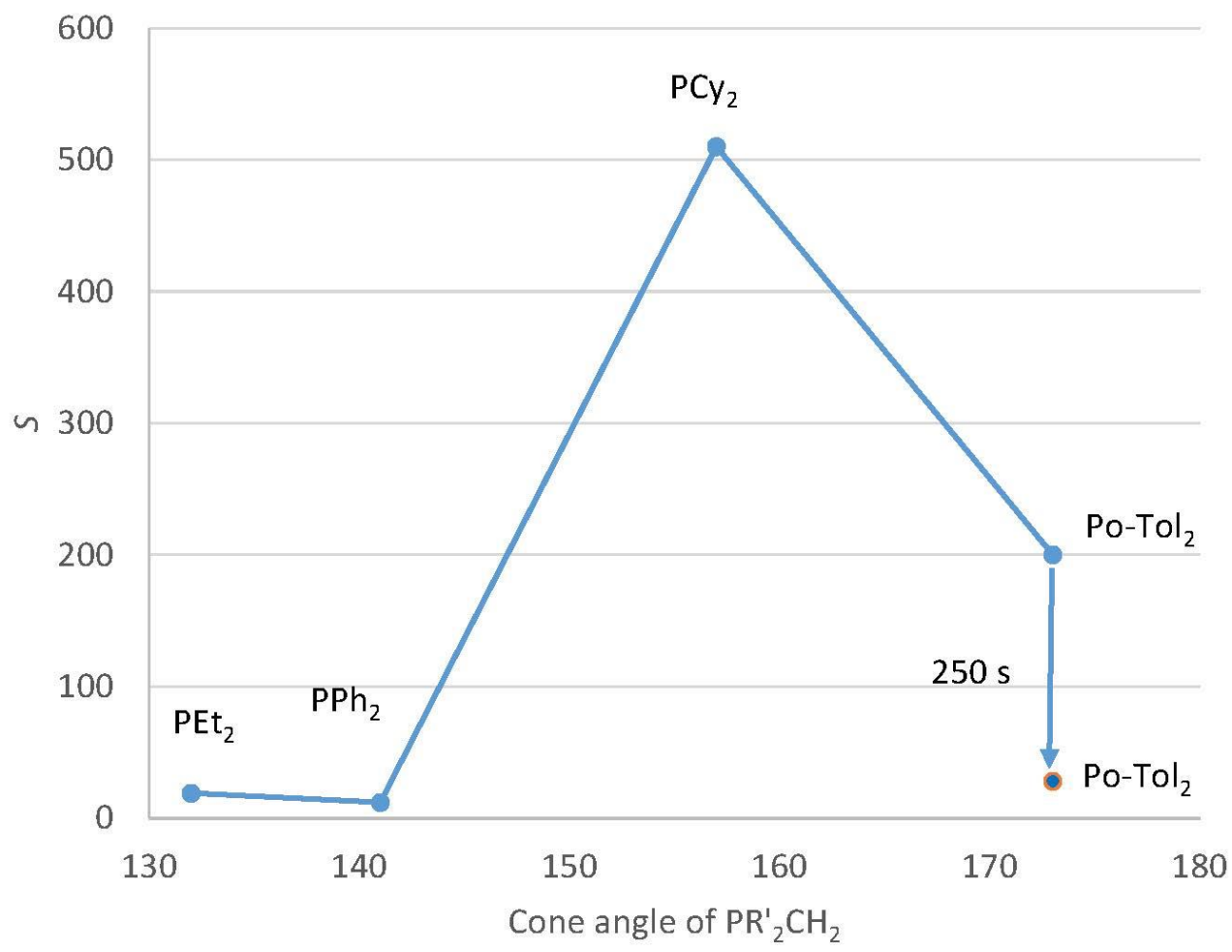

Figure 12. The intrinsic enantioselectivity $S=k_{R} / k_{S}$ for the ATH of acetophenone catalyzed by $(S, S)-1$ to $(S, S)-2$ and $\mathrm{C} 1$ and $\mathrm{C} 4$ as a function of cone angle $\left({ }^{\circ}\right)$ of the $\mathrm{PR}_{2}{ }_{2} \mathrm{CH}_{2}$ group. Reaction conditions are as in Figure 6 . The selectivity of $(\boldsymbol{S}, \boldsymbol{S})-\mathbf{2}$ drops over the first $250 \mathrm{~s}$ of the reaction.

\subsubsection{Other substrates}

The ATH of a range of ketones shown in Figure 13 were tested for the complexes, $(\mathbf{S}, \mathbf{S})-\mathbf{1}$ and $(S, S)-2$ (Figure ). All of the catalytic results from this study were compared to those of $\mathbf{C 4}$, and those of $\mathbf{C 1}$, retested under the conditions of the present study for ease of comparison. 
$\overbrace{H}^{O}$

A1<smiles>CCC(=O)c1ccccc1</smiles>

K1<smiles>CCC(=O)c1ccccc1</smiles>

K2<smiles>CC(C)C(=O)c1ccccc1</smiles>

K3<smiles>CC(=O)c1ccc(Cl)cc1</smiles>

K4<smiles>CC(=O)c1ccccc1Cl</smiles>

K5<smiles>CC(=O)c1cc(C(F)(F)F)cc(C(F)(F)F)c1</smiles>

K6<smiles>CC(=O)c1ccc(C)cc1</smiles>

K7<smiles>CC(=O)c1ccccn1</smiles>

K8<smiles>CC(=O)c1ccco1</smiles>

K9<smiles>CCCC(=O)C(C)C</smiles>

K10<smiles>O=C1CCCCC1</smiles>

K11<smiles>CC(=O)c1ccc2ccccc2c1</smiles>

K12<smiles>O=C(c1ccccc1)C1CCCCC1</smiles>

K13<smiles>O=C(c1ccccc1)c1ccccc1</smiles>

K14<smiles>CC(=O)/C=C/c1ccccc1</smiles>

K15

Figure 13. Substrate scope for ATH using complexes $(S, S)-\mathbf{1},(S, S)-2, C 1$ and $\mathbf{C 4}$.

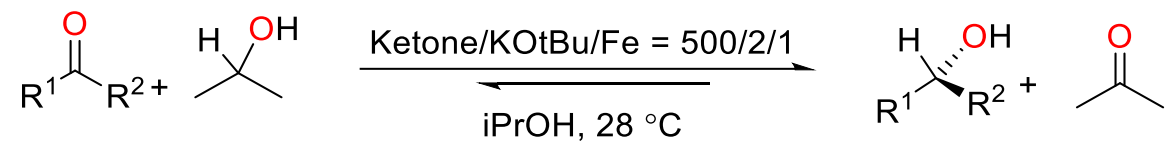

Figure 14. Conditions of ATH catalysis.

The best results are summarized in Table 4; a more complete Table can be found in the SI. Included are results for $\mathbf{C} 4$ as reported elsewhere. ${ }^{[3]}$ All of the chiral alcohol products are enriched in the $R$-enantiomer which is consistent with our previous findings. ${ }^{[3 b, 3 c, 15]}$

First the series of substrates with one phenyl group and one $\mathrm{R}$ group of increasing size ( $\mathrm{H}$ to $\mathrm{Me}$ to Et to $\mathrm{iPr}$ ) are considered. All of the complexes quantitatively convert benzaldehyde (A1) to benzyl alcohol within a few minutes. The reduction of propiophenone, $\mathbf{K} 2$, is achieved with around $80 \%$ conversion for each complex. Complexes $\mathbf{C 4}$ and $(\mathbf{S}, \mathbf{S})-\mathbf{2}$ with the bulky substituents make product with ee greater than $90 \%$ ee while $(S, S)-1$ is less enantioselective (86\% ee) but much more active; $\mathbf{C 1}$ produces a low ee product. The bulkier $\mathbf{K 3}$, i-butyrophenone, is only 
reduced by $(S, S)-1$ and $\mathbf{C} 1$ with the smaller $\mathrm{PR}_{2}^{\prime}$ substituents $\left(\theta<142^{\circ}\right)$ but the enantioselectivity is poor; this suggests that the active sites of the other catalysts are too restricted in size.

Table 4. Results for the ATH of ketones in Figure 13 using complexes $\mathbf{C 1},(\mathbf{S}, \mathbf{S})-\mathbf{1}$ to $(\mathbf{S}, \mathbf{S})-\mathbf{2}$, and previously reported results using $\mathbf{C 4}^{\text {[a] }}$

\begin{tabular}{|c|c|c|c|c|c|c|}
\hline Entry & Substrate ${ }^{[b]}$ & Precatalyst & Conv. $(\%)^{[c]}$ & Time (min) & ee $(\%)^{[c]}$ & TON \\
\hline 1 & \multirow[t]{3}{*}{ K2 } & $(S, S)-2$ & 82 & 30 & 90 & 410 \\
\hline 2 & & C4 & 80 & 60 & 94 & 400 \\
\hline 3 & & $(S, S)-1$ & 79 & 3 & 86 & 395 \\
\hline 4 & \multirow[t]{2}{*}{ K3 } & $(S, S)-1$ & 52 & 60 & 12 & 260 \\
\hline 5 & & C1 & 88 & 40 & 40 & 440 \\
\hline 6 & \multirow[t]{2}{*}{ K4 } & C4 & 92 & 10 & 52 & 460 \\
\hline 7 & & $(S, S)-2$ & 92 & 10 & 58 & 460 \\
\hline 8 & \multirow[t]{3}{*}{ K5 } & $(S, S)-1$ & $>99$ & 1 & 90 & 500 \\
\hline 9 & & C4 & $>99$ & 10 & 94 & 500 \\
\hline 10 & & C1 & $>99$ & 1 & 94 & 500 \\
\hline 11 & \multirow[t]{2}{*}{ K6 } & $(S, S)-2$ & $>99$ & 30 & 93 & 500 \\
\hline 12 & & C1 & $>99$ & 1 & 91 & 500 \\
\hline 13 & \multirow[t]{2}{*}{ K7 } & $(S, S)-2$ & 60 & 40 & 63 & 300 \\
\hline 14 & & C4 & 60 & 20 & 65 & 300 \\
\hline 15 & \multirow[t]{3}{*}{ K8 } & $(S, S)-1$ & $>99$ & 3 & $\mathrm{~N} / \mathrm{A}$ & 500 \\
\hline 16 & & $(S, S)-2$ & $>99$ & 5 & $\mathrm{~N} / \mathrm{A}$ & 500 \\
\hline 17 & & C1 & $>99$ & 2 & $\mathrm{~N} / \mathrm{A}$ & 500 \\
\hline 18 & K9 & $(S, S)-1$ & 0 & 60 & N/A & 0 \\
\hline 19 & \multirow[t]{3}{*}{ K10 } & C4 & 44 & 60 & 43 & 220 \\
\hline 20 & & $(S, S)-2$ & 75 & 40 & 55 & 375 \\
\hline 21 & & C1 & 90 & 20 & 23 & 450 \\
\hline 22 & \multirow[t]{3}{*}{ K11 } & $(S, S)-1$ & 33 & 60 & $\mathrm{~N} / \mathrm{A}$ & 165 \\
\hline 23 & & $(S, S)-2$ & $>99$ & 2 & $\mathrm{~N} / \mathrm{A}$ & 500 \\
\hline 24 & & C1 & $>99$ & 50 & $\mathrm{~N} / \mathrm{A}$ & 500 \\
\hline 25 & \multirow[t]{3}{*}{ K12 } & $(S, S)-1$ & 81 & 30 & 23 & 405 \\
\hline 26 & & C4 & 88 & 40 & 82 & 440 \\
\hline 27 & & $(S, S)-2$ & 84 & 40 & 59 & 420 \\
\hline 28 & K13 & C4 & 11 & 60 & 53 & 55 \\
\hline 29 & \multirow[t]{4}{*}{ K14 } & $(S, S)-1$ & 90 & 20 & $\mathrm{~N} / \mathrm{A}$ & 450 \\
\hline 30 & & C4 & 89 & 20 & $\mathrm{~N} / \mathrm{A}$ & 445 \\
\hline 31 & & $(S, S)-2$ & 91 & 20 & $\mathrm{~N} / \mathrm{A}$ & 455 \\
\hline 32 & & C1 & 89 & 1 & $\mathrm{~N} / \mathrm{A}$ & 445 \\
\hline 33 & K15 & $(S, S)-2$ & 22 & 30 & 46 & 110 \\
\hline
\end{tabular}

[a] Reaction conditions: $28 \mathrm{C}, 8.9 \times 10^{-3} \mathrm{mmol}$ catalyst, $0.18 \mathrm{mmol} \mathrm{KOtBu}, 44.3 \mathrm{mmol}$ substrate, $6 \mathrm{~mL}$ iPrOH. [b] C/B/S=1/2/500. [c] $\%$ conversion and $\%$ ee determined by chiral GC.

The series of methylketones MeCOAr (K4 to K9) provide a range of functional groups to test the catalysts. The reduction of $\mathbf{K} \mathbf{4}$ and $\mathbf{K 5}$ with chloro substituents on the phenyl ring is achieved with 
high conversions, with the ortho-chloro substituent in $\mathbf{K 5}$ producing much higher ee $(84-94 \%$ depending on the catalyst) than with the para-chloro group in $\mathbf{K 4}$ (6-58\% ee). High enantiopurity is beneficial for the production of $(R)-2^{\prime}$-chloro-1-phenylethanol as it is a key intermediate for a chemotherapeutic drug. ${ }^{[16]}$ The bis- $\mathrm{CF}_{3}$-substituted arylketone $\mathbf{K} \mathbf{6}$ is fully reduced to the alcohol in $>90 \%$ ee by $\mathbf{C} \mathbf{1}$ and $(\boldsymbol{S}, S)-\mathbf{2}$ that contain $\mathrm{PAr}_{2}$ groups while $(\boldsymbol{S}, \boldsymbol{S})-\mathbf{1}$ with a more basic $\mathrm{PEt}_{2}$ group appears to be deactivated by the acidic alcohol product after $40-60 \%$ conversion. The enantiopure alcohol is valuable as it is a key intermediate for the synthesis of Aprepitant. ${ }^{[17]}$ Paramethylacetophenone (K7) was more difficult to reduce than the chloro analogue, and relatively low ee are achieved. The presence of a potentially coordinating pyridyl group in $\mathbf{K} \mathbf{8}$ is conducive, not detrimental, to the complete reduction of the ketone. However, the furan in $\mathbf{K} \mathbf{9}$ deactivates all of the complexes except $\mathbf{C 1} .{ }^{[3 b]} 3$-Methyl-2-butanone (K10) is reduced to $90 \%$ conversion but with low ee by $\mathbf{C} \mathbf{1}$, while both $\mathbf{C} 4$ and $(\mathbf{S}, \mathbf{S})-\mathbf{2}$ are less active but give higher ee at $43 \%$ and $55 \%$, respectively. The complete reduction of cyclohexanone (K11) is achieved with $(\mathbf{S}, \mathbf{S})-\mathbf{2}$ and $\mathbf{C 1}$, while only partial conversion is observed for $(\mathbf{S}, \mathbf{S})$-1. $\mathbf{C 4}$ is the most enantioselective catalyst for the ATH of the 2-naphthyl ketone $\mathbf{K 1 2}$ with $82 \%$ ee, and it was the only complex that successfully reduced cyclohexylphenyl ketone (K13), although with only $11 \%$ conversion. All of the catalysts reduce benzophenone (K14) efficiently. The presence of a $\mathrm{C}=\mathrm{C}$ bond in benzylidene acetone (K15) proved to be a complicating factor as all of the complexes with the exception of $(\boldsymbol{S}, \boldsymbol{S})$-2 are unselective and reduced both the $\mathrm{C}=\mathrm{O}$ and $\mathrm{C}=\mathrm{C}$ bonds. For the unselective catalysts, the $\mathrm{GC}$ traces show the three possible reduction products of $\mathrm{C}=\mathrm{O}$ reduction, $\mathrm{C}=\mathrm{C}$ reduction, and both, even at $1 \mathrm{~min}$, with the fully reduced product growing in over time. $(S, S)-2$ is selective for the formation of the allyl alcohol product with less than $<1 \%$ conversion to the doubly saturated product after 60 min. In summary, while $\mathbf{C 1}$ is a very active catalyst with a high TOF, replacing one of its $\mathrm{PPh}_{2}$ groups with a $\mathrm{PC}_{2}$ group results in a more enantioenriched alcohol for all of the ketones except K5. The change to a $\mathrm{P}(o-\mathrm{Tol})_{2}$ group gave superior ee for $\mathbf{K 2}, \mathbf{K} 4, \mathbf{K} 6, \mathbf{K} \mathbf{7}$ and $\mathbf{K 1 0}$. Surprisingly even the small $\mathrm{PEt}_{2}$ group of $(S, S)-1$ provided comparable or greater enantioselectivity to that of the $\mathrm{PPh}_{2}$ group in complex $\mathbf{C 1}$ for K2, K4, K7, K10, K12 and K13. 


\subsection{Observation of Hydride Species}

The precatalyst solution of $\mathbf{C 4}$ was treated with base and $\mathrm{iPrOH}$ in order to characterize possible catalytically active species by use of NMR and IR spectroscopy as has been done already for $\mathbf{C 1} .{ }^{[3 b]}$ When $\mathbf{C 1}$ was treated by first the addition of KOtBu in THF, then evaporation, dissolution in $\mathrm{C}_{6} \mathrm{D}_{6}$, a mixture of amido-enamido complexes was identified. ${ }^{[3 \mathrm{~b}]}$ Then the addition of iPrOH produced first, a transient hydride isomer G1-1 (Scheme 2) which rearranged over time to a second isomer

G1-2. Based on NOE studies, isomer G1-1 has a trans configuration with the Fe-H group parallel to the $\mathrm{N}-\mathrm{H}$ group while $\mathbf{G 1 - 2}$ is thought to have the trans configuration with Fe-CO moiety parallel to

the

$\mathrm{N}-\mathrm{H}$

group. ${ }^{[3 b]}$

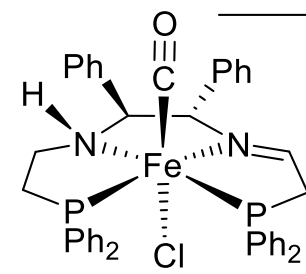

C1
$\left[\mathrm{BPh}_{4}\right]$

1) 8 eq. $\mathrm{KOtBu} 2) \mathrm{iPrOH}$

THF

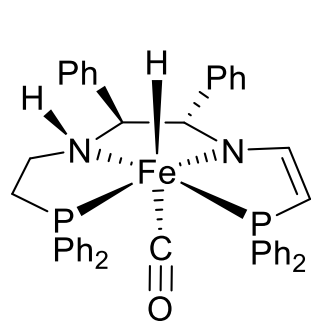

G1-1

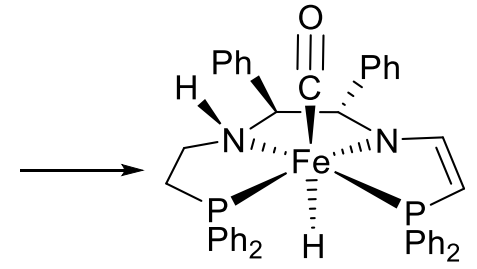

G1-2

Scheme 2. Generation of Iron(II) Hydride Species Starting with C1 as Reported Previously

The precatalyst $\mathrm{C} 4$ was mixed with 8 equivalents of KOtBu in THF for $5 \mathrm{~min}$, then dried in vacuo and dissolved in $\mathrm{C}_{6} \mathrm{D}_{6}$. The solution at this stage produce complicated NMR spectra with features reported previously for amido-eneamido complexes generated from C1 (see the Supporting Material). ${ }^{[3 b, 7,15]}$ The mixture of complexes was then dissolved and stirred in $\mathrm{PrOH}$ for $1 \mathrm{~min}$, or until the residue was completely dissolved. It was then dried immediately affording the new hydride species trans-(S,S)-3 (Scheme 3, Table 5). Its NMR properties are similar to those of hydride G-1-2 and so we tentatively assign the structure as trans with the $\mathrm{NH}$ parallel to the FeCO group. The ${ }^{31} \mathrm{P}\left\{{ }^{1} \mathrm{H}\right\}$ NMR resonances associated with these hydride complexes have ${ }^{2} J_{\mathrm{PP}} 25-27 \mathrm{~Hz}$, consistent with cis phosphorus nuclei (Table 5). G-1-2 and trans-(S,S)-3 show a similar doublet of doublet hydride pattern in the region of -9.3 to $-10.3 \mathrm{ppm}$ with ${ }^{2} J_{\mathrm{PH}}$ of $59-60$ and $80-82 \mathrm{~Hz}$. These coupling constants are typical of cis phosphorus and hydride nuclei on Fe(II) and thus the stereochemistry of these hydride complexes is likely to be trans. In all cases found so far, the magnitude of ${ }^{2} J_{\mathrm{P}-\mathrm{Fe}-\mathrm{H}^{\text {trans }}}$ is smaller than that of ${ }^{2} \mathrm{~J}_{\mathrm{P}-\mathrm{Fe}-\mathrm{H}}{ }^{\text {is }}$ for terminal iron hydride complexes: ${ }^{2} \mathrm{~J}_{\mathrm{P}-}$ 
Fe- $\mathrm{H}^{\text {trans }}$ is in the range $12-35 \mathrm{~Hz}^{[18]}$ whereas ${ }^{2} \mathrm{~J}_{\mathrm{P}-\mathrm{Fe}-\mathrm{H}^{\mathrm{cis}}}$ is in the range $30-85 \mathrm{~Hz}$. ${ }^{[18 \mathrm{a}-\mathrm{e}, 18 \mathrm{i}, 19]}$ In some instances the ${ }^{2} J_{\mathrm{P}-\mathrm{Fe}-\mathrm{H}}$ trans and ${ }^{2} \mathrm{~J}_{\mathrm{P}-\mathrm{Fe}-\mathrm{H}}$ is in iron hydride polyphosphine complexes are averaged to a small value due to nuclear exchange caused by molecular fluxionality. ${ }^{[20]}$

Treating $(\mathbf{S}, \mathbf{S})-\mathbf{1}$ and $(\mathbf{S}, \mathbf{S})-\mathbf{2}$ in similar fashion also produce hydride resonances in this region but the hydrides are very reactive and unstable so that the ${ }^{31} \mathrm{P}\left\{{ }^{1} \mathrm{H}\right\}$ NMR spectra are complex with some unidentified species and signals for uncoordinated phosphorus species.

Thus hydride complex trans-(S,S)-3, like G1-2, is thought to have the NH of the ligand parallel to the Fe-CO group and not in a suitable position for an outersphere hydride and proton attack on the ketone. ${ }^{[3 b]}$
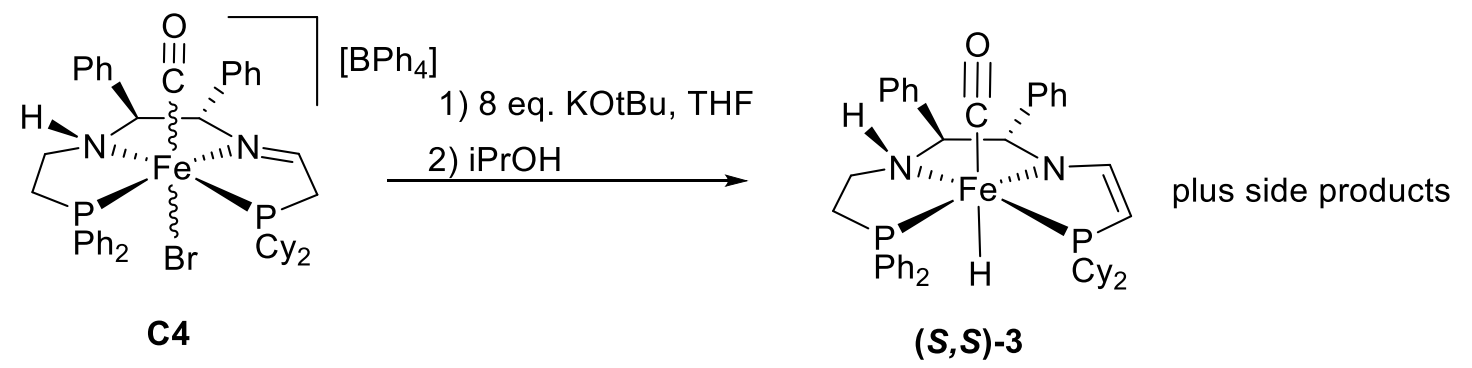

Scheme 3. Generation of Iron(II) Hydride Species $(S, S)-3$

Table 5. NMR properties of the hydride complexes

\begin{tabular}{|c|c|c|c|c|c|}
\hline $\begin{array}{l}\text { Precursor } \\
\text { to Hydride } \\
\text { Complex }\end{array}$ & $\mathrm{PR}_{2}^{\prime}$ & $\delta^{31} \mathrm{P}(\mathrm{ppm})$ & ${ }^{2} J_{P P}(H z)$ & ${ }^{\delta 1} \mathrm{H}(\mathrm{ppm})$ & ${ }^{2} J_{H P}(H z)$ \\
\hline $\begin{array}{l}\text { C1 hydride } \\
\text { G-1-1 }\end{array}$ & $\mathrm{PPh}_{2}$ & $84.9,70.4$ & 33.2 & -2.3 & $71,62^{a}$ \\
\hline $\begin{array}{l}\text { C1 hydride } \\
\text { G-1-2 }\end{array}$ & $\mathrm{PPh}_{2}$ & $75.7,71.4$ & 27.5 & -9.2 & $80,60^{b}$ \\
\hline$(S, S)-3$ & $\mathrm{PC}_{2}$ & $91.2,83.0$ & 36.6 & -10.3 & $82.2,59.8$ \\
\hline
\end{tabular}

\section{Density Functional Theory Calculations}

Due to the difficulties in establishing the coordination geometries of the products upon reacting C4 with base, we employed Density Functional Theory to compare relative ground state energies 
of various diastereomers. Details of the calculations and the three dimensional Cartesian coordinates are provided in the Supporting Information. The calculated geometries of cis- $\beta$

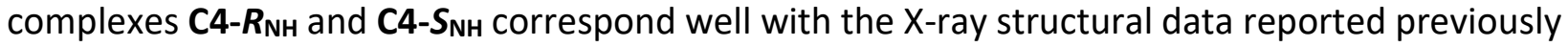
for $\mathrm{C4}_{,}{ }^{[8]}$ with $\mathbf{C} 4-\mathrm{S}_{\mathrm{NH}}$ being only $1.8 \mathrm{kcal} / \mathrm{mol}$ higher in energy (SI, Scheme S1). The cis- $\beta$ stereochemistry of the two diastereomers can be further characterized as $\Delta$-cis- $\beta$-C4- $\boldsymbol{R}_{\mathrm{NH}}$ and $\Lambda$ cis- $\beta$-C4-S $S_{\mathrm{NH}}$. Deprotonation of $\mathbf{C 4}$ with at least two equivalents of base could lead to the formation of at least four possible amido-eneamido complexes (S,S)-4 (Scheme 4 ) and the transamido structure by analogy to mechanistic studies based on $\mathbf{C 1}^{[15,21]}$ We found that the trans$(\mathbf{S}, \mathbf{S})-\mathbf{4}$ isomer is slightly lower in energy $(3.2 \mathrm{kcal} / \mathrm{mol})$ relative to the $\Delta$-cis- $\beta$ - $(\mathbf{S}, \mathbf{S})-\mathbf{4}$ amido complex (Scheme 4). The trans isomer can produce, via the transfer of a proton/hydride equivalent from $\mathrm{iPrOH}$, the high energy, catalytically active, octahedral, FeH-NH complex, trans$(\mathbf{S}, \mathbf{S})-\mathbf{3}^{\prime}$, with FeH and $\mathrm{NH}$ parallel as proposed for the trans-amido-eneamido from $\mathbf{C}$. In the absence of substrate, the formation of the hydride $\Delta-c i s-\beta-(S, S)-3$ is calculated to be most favorable $(-13.3 \mathrm{kcal} / \mathrm{mol})$ among the various cis- $\beta$ structures. Experimentally the hydride trans$(\mathbf{S}, \mathbf{S})-\mathbf{3}$ is the isomer observed as noted above. It is calculated to have a relative energy of -10.9 $\mathrm{kcal} / \mathrm{mol}$, or $2.4 \mathrm{kcal} / \mathrm{mol}$ less stable than this $\Delta$-cis- $\beta$ isomer although this difference may be within the errors of the calculation. The relative energy of the kinetically-formed hydride complex trans-(S,S)-3' is calculated to be $-8.9 \mathrm{kcal} / \mathrm{mol}, 4.4 \mathrm{kcal} / \mathrm{mol}$ higher than the $\Delta$-cis- $\beta$ isomer and $2 \mathrm{kcal} / \mathrm{mol}$ higher than the observed hydride $(\mathbf{S}, \mathbf{S})-\mathbf{3}$. 

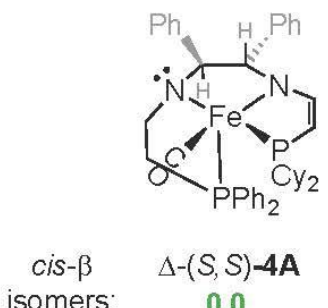

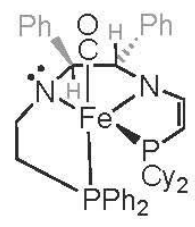

$\Delta-(S, S)-4 B$

14.8

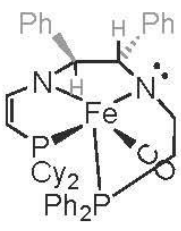

$\Lambda-(S, S)-4 A$

6.9

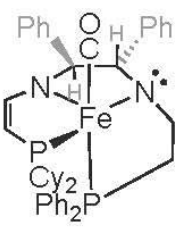

$\Lambda-(S, S)-4 B$

19.1

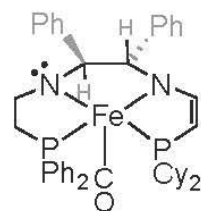

trans-(S,S)-4

$-3.2$

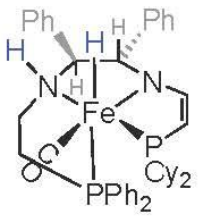

cis- $\beta$

isomers:
$\Delta-(S, S)-3 A$
-13.3

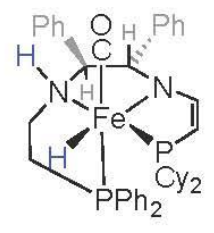

$\Delta-(S, S)-3 B$

$-8.8$

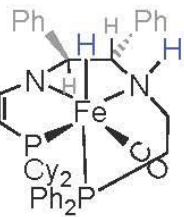

$\Lambda-(S, S)-3 A$ $-10.0$

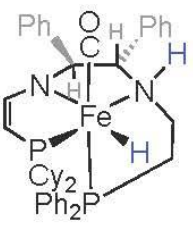

$\Lambda-(S, S)-3 \mathbf{B}$ $-6.5$

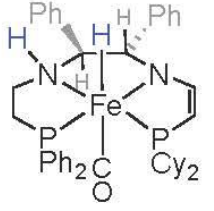

trans-(S,S)-3' $-8.9$

Scheme 4. Ground state energy comparison of some isomers of Cy-amido complexes $(S, S)-4$ and the hydride isomers $(\boldsymbol{S}, \boldsymbol{S})-3$ derived from them by reaction with 2-PrOH. All energies are given in $\mathrm{kcal} / \mathrm{mol}$ and relative to $\Delta$-cis- $\beta-(S, S)-4$ plus relevant small molecules. M06L/TZVP/TZVPfit-IEF-PCM(THF).

\section{Proposed mechanism}

Thus, we suggest that the activity of the system is determined by the relative stability of a catalytic trans-hydride like trans-(S,S)-3' and the off cycle trans hydride trans-(S,S)-3 or $\Delta$-cis- $\beta$ (S,S)-3 hydride species and of the catalytic trans amido-eneamido species as indicated in Scheme 5. The larger and/or more basic $\mathrm{PR}_{2}$ groups favor the off-cycle structures and thus are less reactive. The similarity of the structure of the ketone adduct with the unobserved active hydride (Scheme 5) to that of the one proposed for the $\mathrm{PPh}_{2}$ catalyst system $\mathbf{C 1}^{[15,21]}$ could explain why the $(R)$-aryl alcohols are produced by the trans-(S,S)-hydride catalysts using a transition state structure similar to that described for $\mathbf{C 1} .{ }^{[15,21]}$

DFT calculations (see SI) indicate that G-1-2 may have a $\Delta$-cis- $\beta$ geometry for the off cycle structure shown in Scheme 5; this structure is $3 \mathrm{kcal} / \mathrm{mol}$ more stable than the trans-isomer $\mathbf{G - 1 - 1}$. Thus it is 
possible that the off-cycle hydride complexes with a $\Delta$-cis- $\beta$ geometry rearrange to low concentration catalytically active isomers with the $\mathrm{NH}$ and FeH parallel as shown in Scheme 5.

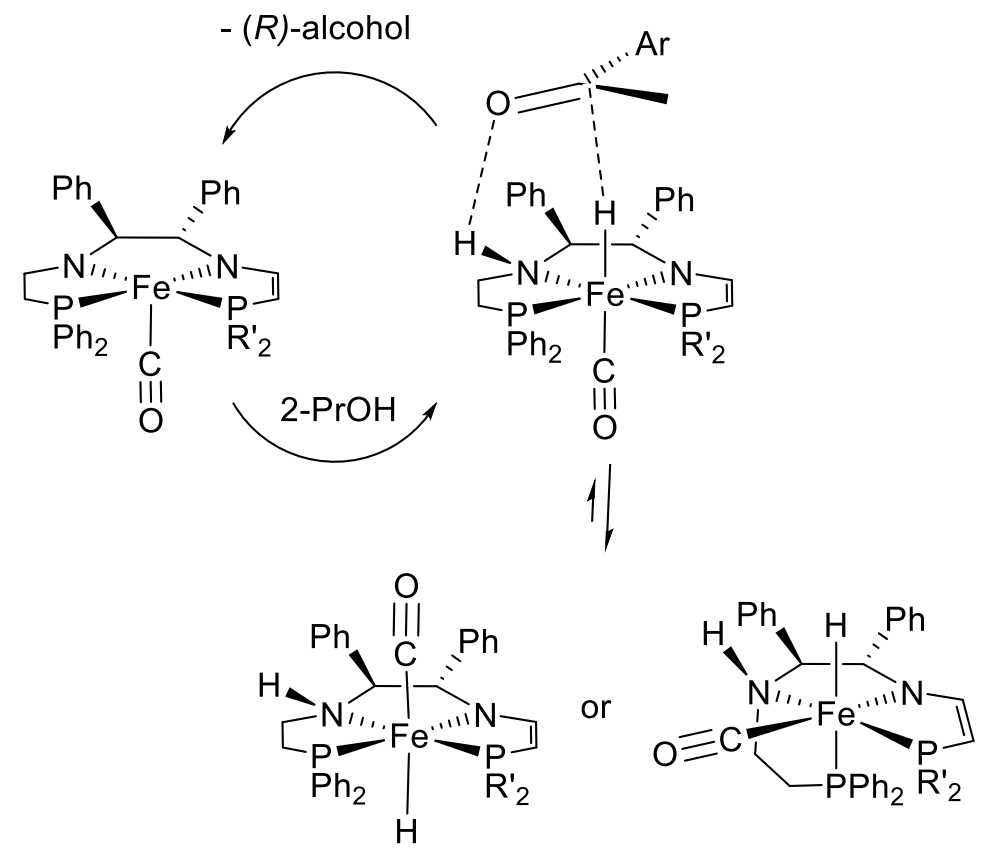

Scheme 5. Proposed mechanism for the ATH catalyzed by complexes $(\mathbf{S}, \mathbf{S})-\mathbf{1},(\mathbf{S}, \mathbf{S})-\mathbf{2}, \mathbf{C} \mathbf{1}$ and $\mathbf{C 4}$. The observed hydride trans-(S,S)-3 when $\mathrm{R}^{\prime}$ is $\mathrm{Cy}$ is proposed to be off cycle.

\section{Summary}

In conclusion, two new precatalyst complexes have been synthesized with varying steric and electronic properties at one phosphine ( $\mathrm{PEt}_{2}$ vs $\left.\mathrm{Po}_{-} \mathrm{Tol}_{2}\right)$ coordination site of our third generation iron(II) (P-NH-N-P') system. These have been tested in the catalytic ATH of ketones and compared to the previously reported complexes $\mathbf{C} \mathbf{1}$ and $\mathbf{C}$. The progress of the catalytic reactions could be fit for the first time to a simple three parameter kinetic model which describes the activity and degradation of ee over the course of the reaction. We found that by increasing the steric bulk at one phosphine, the enantioselectivity increases (to greater than $98 \%$ in case of C4) with a concomitant decrease in activity.

This work provides evidence for a correct matching of catalyst structure with substrate structure to produce superior activity and selectivity. The bulky isobutyrophenone (K3) is only reduced by $(\mathbf{S}, \mathbf{S})-\mathbf{1}$ and $\mathbf{C 1}$ probably because these catalysts have the small $\mathrm{PEt}_{2}$ or $\mathrm{PPh}_{2}$ groups. Surprisingly 
$(\mathbf{S}, \mathbf{S})$-1 provides $(R)$-1-orthochlorophenylethanol in high ee $(90 \%)$ from $\mathbf{K 5}$, despite the small size of the $\mathrm{PEt}_{2}$ group. Cyclohexanone (K11) was only reduced by the $(\mathbf{S}, \mathbf{S})-\mathbf{2}$ and $\mathbf{C} \mathbf{1}$ catalyst systems. We also found that the steric and electronic properties can be varied to introduce chemoselectivity, as in the case of the selective $\mathrm{C}=\mathrm{O}$ reduction of benzylidene acetone by complex (S,S)-2 and none other.

We provided spectroscopic and computational characterization of hydride complexes that provide an understanding of the mechanism of the catalytic ATH using these iron complexes.

\section{Acknowledgements}

NSERC Canada is thanked for a Discovery Grant to RHM and scholarship funding to DEP. SAM thanks Digital Specialty Chemicals Ltd. for an OGSST Scholarship. The authors acknowledge the Canadian Foundation of Innovation, project number 19119, and the Ontario Research Fund for

funding of the Centre for Spectroscopic Investigation of Complex Organic Molecules and Polymers and Compute Canada for providing computational resources.

\section{References}

[1] a) L. Cotarca, M. Verzini, R. Volpicelli, Chimica Oggi-Chem. Today 2014, 32, 36-41; b) P. Dupau, in Organometallics as Catalysts in the Fine Chemical Industry, Vol. 42 (Eds.: M. Beller, H. U. Blaser), Springer-Verlag, Berlin, 2012, pp. 47-63; c) R. Noyori, Adv. Synth. Catal. 2003, 345, 15-32; d) L. A. Saudan, Acc. Chem. Res. 2007, 40, 1309-1319.

[2] a) J. S. Chen, L. L. Chen, Y. Xing, G. Chen, W. Y. Shen, Z. R. Dong, Y. Y. Li, J. X. Gao, Acta Chimica Sinica (Huaxue Xuebao) 2004, 62, 1745-1750; b) R. Bigler, R. Huber, A. Mezzetti, Synlett 2016, 27, 831-847; c) R. Bigler, A. Mezzetti, Org. Process Res. Dev. 2016, 20, 253-261; d) T. Bleith, H. Wadepohl, L. H. Gade, J. Am. Chem. Soc. 2015, 137, 2456-2459; e) F. Foubelo, C. Nájera, M. Yus, Tetrahedron Asymmetry 2015, 26, 769-790; f) J. P. Hopewell, J. E. D. Martins, T. C. Johnson, J. Godfrey, M. Wills, Org. Biomol. Chem. 2012, 10, 134-145; g) Y. Y. Li, S. L. Yu, W. Y. Shen, J. X. Gao, Acc. Chem. Res. 2015, 48, 2587-2598; h) R. H. Morris, Chem. Soc. Rev. 2009, 38, 2282-2291; i) R. H. Morris, Acc. Chem. Res. 2015, 48, 1494-1502; j) A. Naik, T. Maji, O. Reiser, Chem. Commun. 2010, 46, 9265-9265; k) B. Stefane, F. Pozgan, Catal. Rev. Sci. Eng. 2014, 56, 82-174; I) P. E. Sues, K. Z. Demmans, R. H. Morris, Dalton Trans. 2014, 43, 7650-7667; m) S. L. Yu, W. Y. Shen, Y. Y. Li, Z. R. Dong, Y. Q. Xu, Q. Li, J. N. Zhang, J. X. Gao, Adv. Synth. Catal. 2012, 354, 818822.

[3] a) A. A. Mikhailine, M. I. Maishan, A. J. Lough, R. H. Morris, J. Am. Chem. Soc. 2012, 134, 1226612280; b) W. Zuo, A. J. Lough, Y. Li, R. H. Morris, Science 2013, 342, 1080-1083; c) S. A. M. Smith, R. H. Morris, Synthesis 2015, 47, 1775-1779; d) R. Bigler, R. Huber, A. Mezzetti, Angew. Chem. 
Int. Ed. 2015, 54, 5171 -5174; e) R. Bigler, A. Mezzetti, Org. Lett. 2014, 16, 6460-6463; f) R.

Bigler, E. Otth, A. Mezzetti, Organometallics 2014, 33, 4086-4099.

[4] C. Sui-Seng, F. Freutel, A. J. Lough, R. H. Morris, Angew. Chem. Int. Ed. 2008, 47, 940-943.

[5] J. F. Sonnenberg, N. Coombs, P. A. Dube, R. H. Morris, J. Am. Chem. Soc. 2012, 134, 5893-5899.

[6] a) P. O. Lagaditis, A. J. Lough, R. H. Morris, Inorg. Chem. 2010, 49, 10057-10066; b) P. E. Sues, A. J. Lough, R. H. Morris, Organometallics 2011, 30, 4418-4431.

[7] W. Zuo, S. Tauer, D. E. Prokopchuk, R. H. Morris, Organometallics 2014, 33, 5791-5801.

[8] S. A. M. Smith, A. J. Lough, R. H. Morris, UICrData 2017, https://doi.org/10.1107/S2414314617004527.

[9] W. Zuo, R. H. Morris, Nature Prot. 2015, 10, 241-257.

[10] A. A. Mikhailine, P. O. Lagaditis, P. Sues, A. J. Lough, R. H. Morris, J. Organometal. Chem. 2010, 695, 1824-1830.

[11] C. A. Tolman, Chem. Rev. 1977, 77, 313-348.

[12] R. Bigler, R. Huber, M. Stöckli, A. Mezzetti, ACS Catalysis 2016, 6, 6455-6464.

[13] L. Hintermann, C. Dittmer, Eur. J. Org. Chem. 2012, 2012, 5573-5584.

[14] P. Kuzmic, Anal. Biochem. 1996, 237, 260.

[15] W. Zuo, D. E. Prokopchuk, A. J. Lough, R. H. Morris, ACS Catal. 2016, 6, 301-314.

[16] T. Eixelsberger, J. M. Woodley, B. Nidetzky, R. Kratzer, Biotechnol. Bioeng. 2013, 100, 23112231.

[17] K. M. J. Brands, J. F. Payack, J. D. Rosen, T. D. Nelson, A. Candelario, M. A. Huffman, M. M. Zhao, J. Li, B. Craig, Z. J. Song, D. M. Tschaen, K. Hansen, P. N. Devine, P. J. Pye, K. Rossen, P. G.

Dormer, R. A. Reamer, C. J. Welch, D. J. Mathre, N. N. Tsou, J. M. McNamara, P. J. Reider, J. Am. Chem. Soc. 2003, 125, 2129 - 2135.

[18] a) C. A. Tolman, S. D. Ittel, A. D. English, J. P. Jesson, J. Am. Chem. Soc. 1978, 100, 4080-4089; b) N. Bampos, L. D. Field, Inorg. Chem. 1990, 29, 587-588; c) C. Bianchini, M. Peruzzini, A. Polo, A. Vacca, F. Zanobini, Gazz. Chim. Ital. 1991, 121, 543-549; d) G. Jia, S. D. Drouin, P. G. Jessop, A. J. Lough, R. H. Morris, Organometallics 1993, 12, 906-916; e) N. Bampos, L. D. Field, B. A. Messerle, Organometallics 1993, 12, 2529-2535; f) D. G. Gusev, R. Huebener, P. Burger, O. Orama, H. Berke, J. Am. Chem. Soc. 1997, 119, 3716-3731; g) D. Schott, P. Callaghan, J. Dunne, S. B. Duckett, C. Godard, J. M. Goicoechea, J. N. Harvey, J. P. Lowe, R. J. Mawby, G. Müller, R. N. Perutz, R. Poli, M. K. Whittlesey, Dalton Trans. 2004, 3218-3224; h) R. J. Trovitch, E. Lobkovsky, P. J. Chirik, Inorg. Chem. 2006, 45, 7252-7260; i) P. Bhattacharya, J. A. Krause, H. Guan, Organometallics 2011, 30, 4720-4729.

[19] a) M. Bautista, K. A. Earl, R. H. Morris, A. Sella, J. Am. Chem. Soc. 1987, 109, 3780-3782; b) M. T. Bautista, K. A. Earl, R. H. Morris, Inorg. Chem. 1988, 27, 1124-1126; c) M. T. Bautista, E. P. Cappellani, S. D. Drouin, R. H. Morris, C. T. Schweitzer, A. Sella, J. Zubkowski, J. Am. Chem. Soc. 1991, 113, 4876-4887; d) E. Rocchini, P. Rigo, A. Mezzetti, T. Stephan, R. H. Morris, A. J. Lough, C. E. Forde, T. P. Fong, S. D. Drouin, J. Chem. Soc., Dalton Trans. 2000, 3591-3602; e) R. Langer, G. Leitus, Y. Ben-David, D. Milstein, Angew. Chem. Int. Ed. 2011, 50, 2120-2124; f) B. Bichler, C. Holzhacker, B. Stoger, M. Puchberger, L. F. Veiros, K. Kirchner, Organometallics 2013, 32, 4114 4121; g) F. Bertini, N. Gorgas, B. Stoger, M. Peruzzini, L. F. Veiros, K. Kirchner, L. Gonsalvi, Acs Catal. 2016, 6, 2889-2893; h) F. Schneck, M. Assmann, M. Balmer, K. Harms, R. Langer, Organometallics 2016; i) S. Elangovan, B. Wendt, C. Topf, S. Bachmann, M. Scalone, A. Spannenberg, H. J. Jiao, W. Baumann, K. Junge, M. Beller, Adv. Synth. Catal. 2016, 358, 820-825.

[20] G. Guilera, G. S. McGrady, J. W. Steed, R. P. L. Burchell, P. Sirsch, A. J. Deeming, New. J. Chem. 2008, 32, 1573-1581.

[21] D. E. Prokopchuk, R. H. Morris, Organometallics 2012, 31, 7375-7385. 
Asymmetric Transfer Hydrogenation of Ketones Using New Iron(II) (P-NH-N-P') Catalysts: Changing the Steric and Electronic Properties at Phosphorus P'

Samantha A. M. Smith, Demyan, E. Prokopchuk and Robert H. Morris

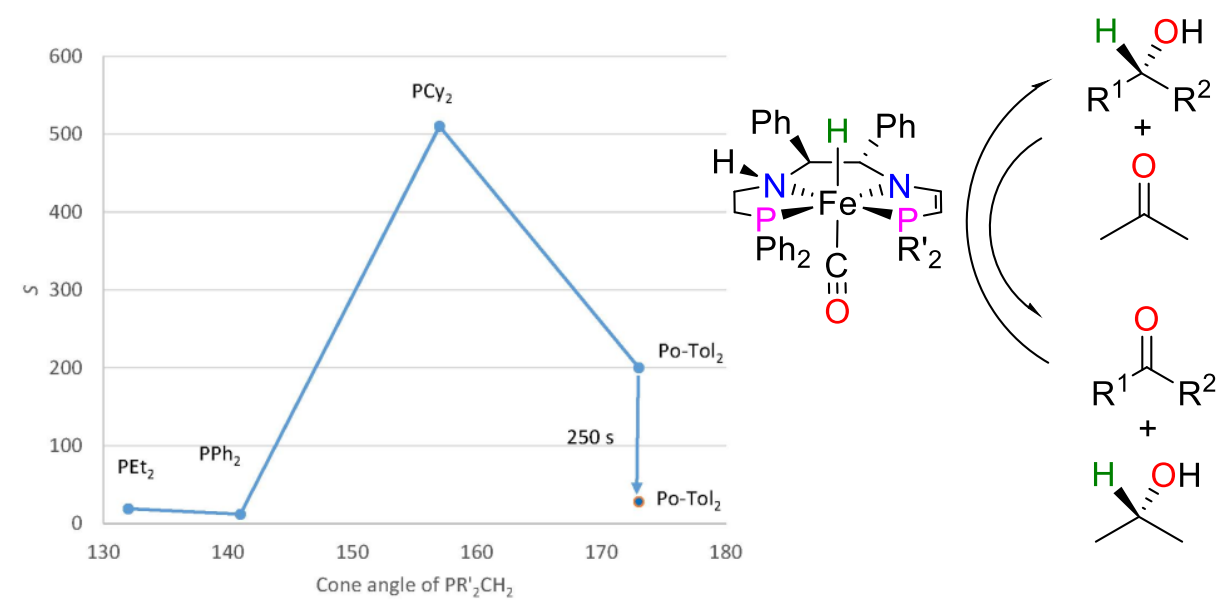


Supporting Information for

Asymmetric Transfer Hydrogenation of Ketones Using New Iron(II) (P-NH-N-P') Catalysts: Changing the Steric and Electronic Properties at Phosphorus $\mathbf{P}^{\prime}$

Samantha A. M. Smith, Demyan E. Prokopchuk, and Robert H. Morris*

*corresponding author. Email: rmorris@chem.utoronto.ca

\section{Contents}

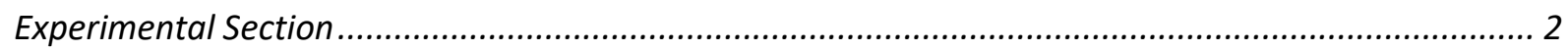

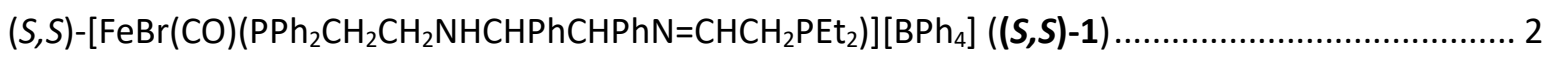

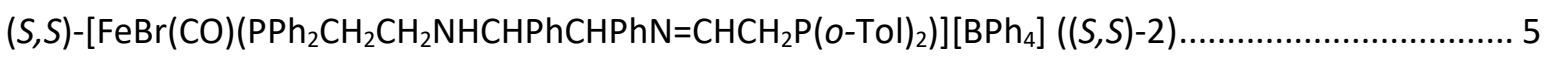

Observation by NMR of the Iron amido-eneamido and hydride

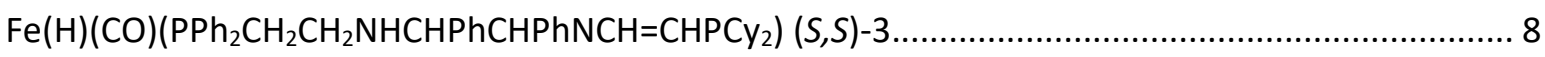

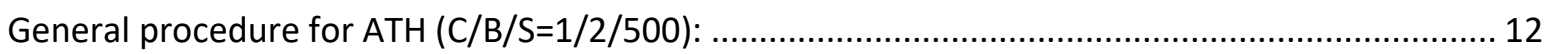

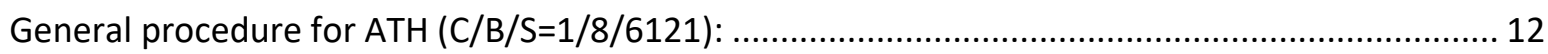

Data for analysis of the structures of the isomers of complexes

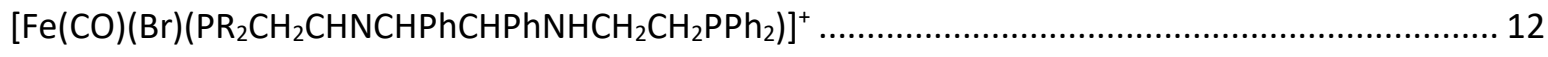

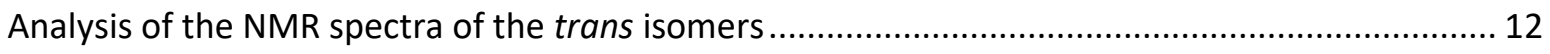

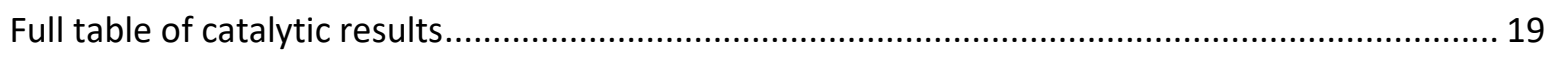

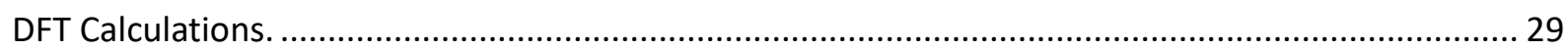




\section{Experimental Section}

All manipulations were done under inert atmosphere of either nitrogen or argon using Schlenk techniques or a glovebox, unless otherwise stated. Solvents were dried and degassed under standard procedures prior to use. NMR spectra were recorded at ambient temperature and pressure using Varian Gemini $600 \mathrm{MHz}, 500 \mathrm{MHz}$ and $400 \mathrm{MHz}$ spectrometers [ ${ }^{1} \mathrm{H}(600 \mathrm{MHz}, 500 \mathrm{MHz}$ and $400 \mathrm{MHz}$ ), ${ }^{13} \mathrm{C}\left\{{ }^{1} \mathrm{H}\right\}(150 \mathrm{MHz}, 125 \mathrm{MHz}$ and $100 \mathrm{MHz})$ and ${ }^{31} \mathrm{P}\left\{{ }^{1} \mathrm{H}\right\}(242 \mathrm{MHz}, 201 \mathrm{MHz}$ and $161 \mathrm{MHz})$. The electrospray ionization mass spectrometry (ESI-MS) data were collected on an AB/Sciex QStar mass spectrometer with an ESI source. IR spectra were measure using a Bruker ALPHA FT-IR-base spectrometer with a DTGS detector, SiC globar source, and automated instrument tests with integrated polystyrene standard.

$(\mathrm{S}, \mathrm{S})-\left[\mathrm{FeBr}(\mathrm{CO})\left(\mathrm{PPh}_{2} \mathrm{CH}_{2} \mathrm{CH}_{2} \mathrm{NHCHPhCHPhN}=\mathrm{CHCH}_{2} \mathrm{PEt}_{2}\right)\right]\left[\mathrm{BPh}_{4}\right]((\mathrm{S}, \mathrm{S})-1)$. In the glovebox, sodium methoxide (64 mg, $1.18 \mathrm{mmol}$ ) was added to a $100 \mathrm{~mL}$ Schlenk flask charged with a stir bar and diethyl-phosphonium bromide (D1) $\left(5.88 \times 10^{-1} \mathrm{mmol}\right)$ and $25 \mathrm{~mL}$ methanol was added with stirring. This solution was stirred for no more than 2 minutes. A solution of dissolved $\left[\mathrm{Fe}\left(\mathrm{H}_{2} \mathrm{O}\right)_{6}\right]\left[\mathrm{BF}_{4}\right]_{2}(496 \mathrm{mg}$, $1.47 \mathrm{mmol}$ ) in $20 \mathrm{~mL}$ acetonitrile was added to the Schlenk flask, followed by a solution of $1.18 \mathrm{mmol}$ of $(S, S)-\mathrm{PPh}_{2} \mathrm{CH}_{2} \mathrm{CH}_{2} \mathrm{NHCH}(\mathrm{Ph}) \mathrm{CH}(\mathrm{Ph}) \mathrm{NH}_{2}$ in $10 \mathrm{~mL}$ methanol. This resulted in a purple solution. This was left to stir at room temperature for 16 hours, after which a colour change to pink or orange was observed. The solvent was removed in vacuo. $\mathrm{KBr}(140 \mathrm{mg}, 4.70 \mathrm{mmol})$ was added to the flask, the flask was sealed and removed from the glovebox. The flask was purged and placed under CO atmosphere using Schlenk techniques. $20 \mathrm{~mL}$ distilled acetone in a syringe was injected into the flask with stirring for 1.5 hours, after which the solvent was removed under vacuum. The residual yellow or orange solid was dissolved in $20 \mathrm{~mL}$ acetone while under $\mathrm{CO}$ atmosphere. This was stirred for 1 additional hour, after which the solvent was removed and the flask brought into the glovebox. The solid residue was dissolved in $20 \mathrm{~mL}$ dichloromethane and filtered through a pad of Celite, then through a $25 \mathrm{~mm}$ Syringe Filter PTFE membrane (pore size $0.45 \mu \mathrm{m}$ ). The clear yellow or orange solution was dried in vacuo, then dissolved in minimal methanol. This solution was added to a vial charged with a stir bar and $\mathrm{NaBPh}_{4}(402 \mathrm{mg}, 1.294 \mathrm{mmol}$ ) dissolved in minimal methanol, from which a yellow solid precipitated. This solid was filtered off and washed with diethyl ether $(3 \times 15 \mathrm{~mL})$ and dried overnight. If the purity by NMR was not sufficient, the solid was dissolved in minimal dichloromethane and precipitated out by addition of diethyl ether. Yield: $42 \mathrm{mg}(35 \%)$. FT-IR ( $\mathrm{KBr}, \mathrm{cm}^{-}$ $\left.{ }^{1}\right): 1956\left(v_{\mathrm{CO}}, w_{1 / 2} 20 \mathrm{~cm}^{-1}\right)$. HRMS (ESI-TOF, $\left.\mathrm{CH}_{2} \mathrm{Cl}_{2}\right) \mathrm{m} / \mathrm{z}$ calculated for $\left[\mathrm{C}_{35} \mathrm{H}_{541} \mathrm{BrFeN}_{2} \mathrm{OP}_{2}\right]^{+}:$701.1145, found: $701.1151^{1} \mathrm{H} N M R\left(300 \mathrm{MHz} ; \mathrm{CD}_{2} \mathrm{Cl}_{2}\right) \delta: 1.03$ (bs, 6H, $\left.\mathrm{P}\left(\mathrm{CH}_{2} \mathrm{CH}_{3}\right)_{2}\right), 1.58$ (bs, $\left.4 \mathrm{H}, \mathrm{P}\left(\mathrm{CH}_{2} \mathrm{CH}_{3}\right)_{2}\right), 2.99$ $\left(\mathrm{m}, 2 \mathrm{H}, \mathrm{CH}_{2} \mathrm{CH}=\mathrm{N}\right), 2.87\left(\mathrm{~m}, 2 \mathrm{H}, \mathrm{CH}_{2} \mathrm{PPh}_{2}\right), 4.00\left(\mathrm{~d}, 2 \mathrm{H}, \mathrm{CH}_{2} \mathrm{NH}, 4.38, \mathrm{~J}_{\mathrm{HH}}=10.9 \mathrm{~Hz}\right), 5.05(\mathrm{t}, 1 \mathrm{H}, \mathrm{NCHPh}$, $\left.J_{\mathrm{HH}}=11.9 \mathrm{~Hz}\right), 5.52(\mathrm{~m}, 1 \mathrm{H}, \mathrm{NCHPh}), 6.83-7.56(\mathrm{~m}, 40 \mathrm{H}, \mathrm{ArH}), 7.79(\mathrm{~m}, 1 \mathrm{H}, \mathrm{N}=\mathrm{CH})$. The air sensitivity of this compound prevented the measuring of acceptable elemental analysis. The spectroscopic evidence and reproducible catalytic activity of separate batches provide support for acceptable purity. 


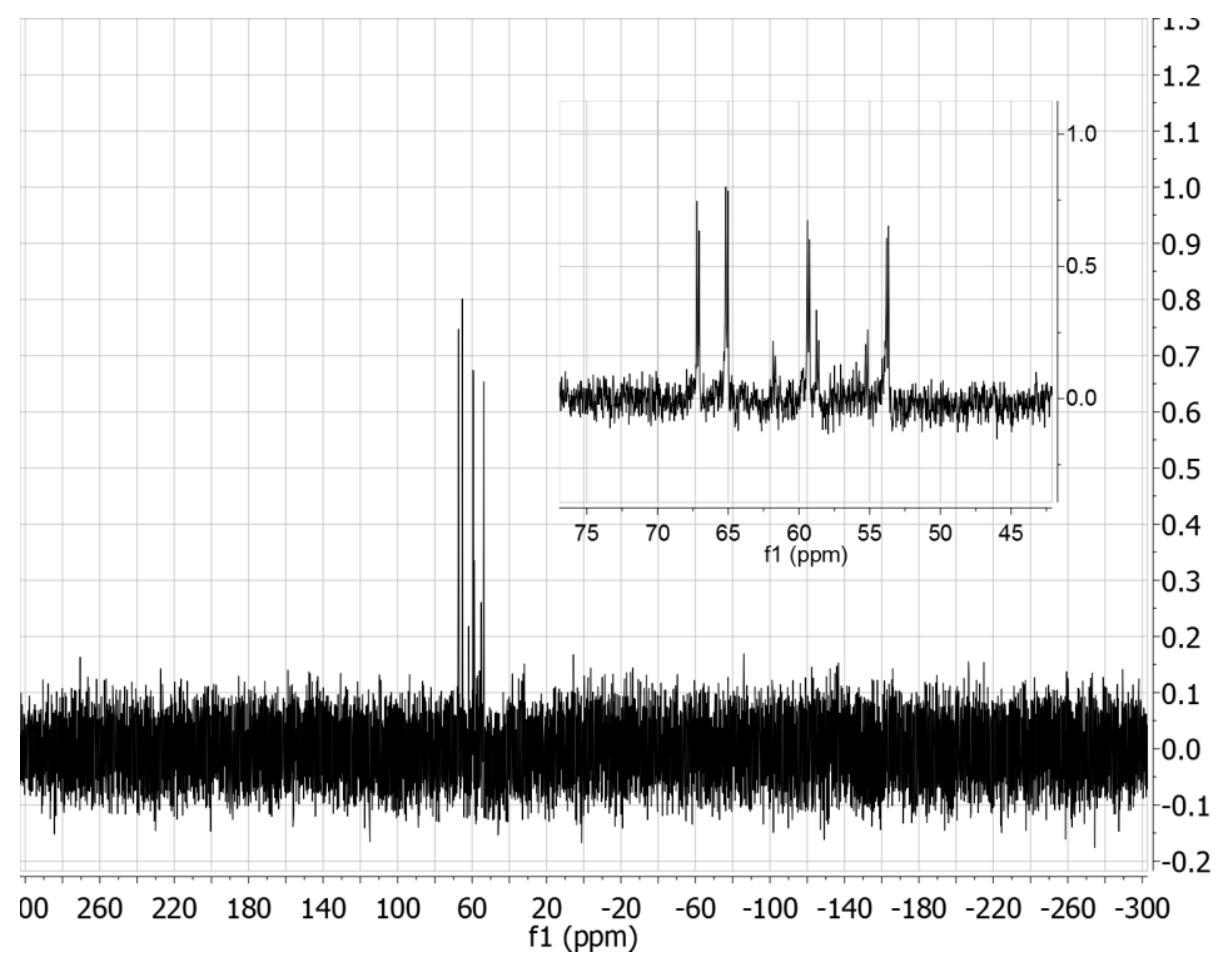

Figure $\mathbf{S 1}{ }^{31} \mathrm{P}\left\{{ }^{1} \mathrm{H}\right\} \mathrm{NMR}\left(242 \mathrm{MHz}, \mathrm{CD}_{2} \mathrm{Cl}_{2}\right)$ spectrum of $(S, S)-1 \mathrm{P} R_{2}=\mathrm{PEt} t_{2}$

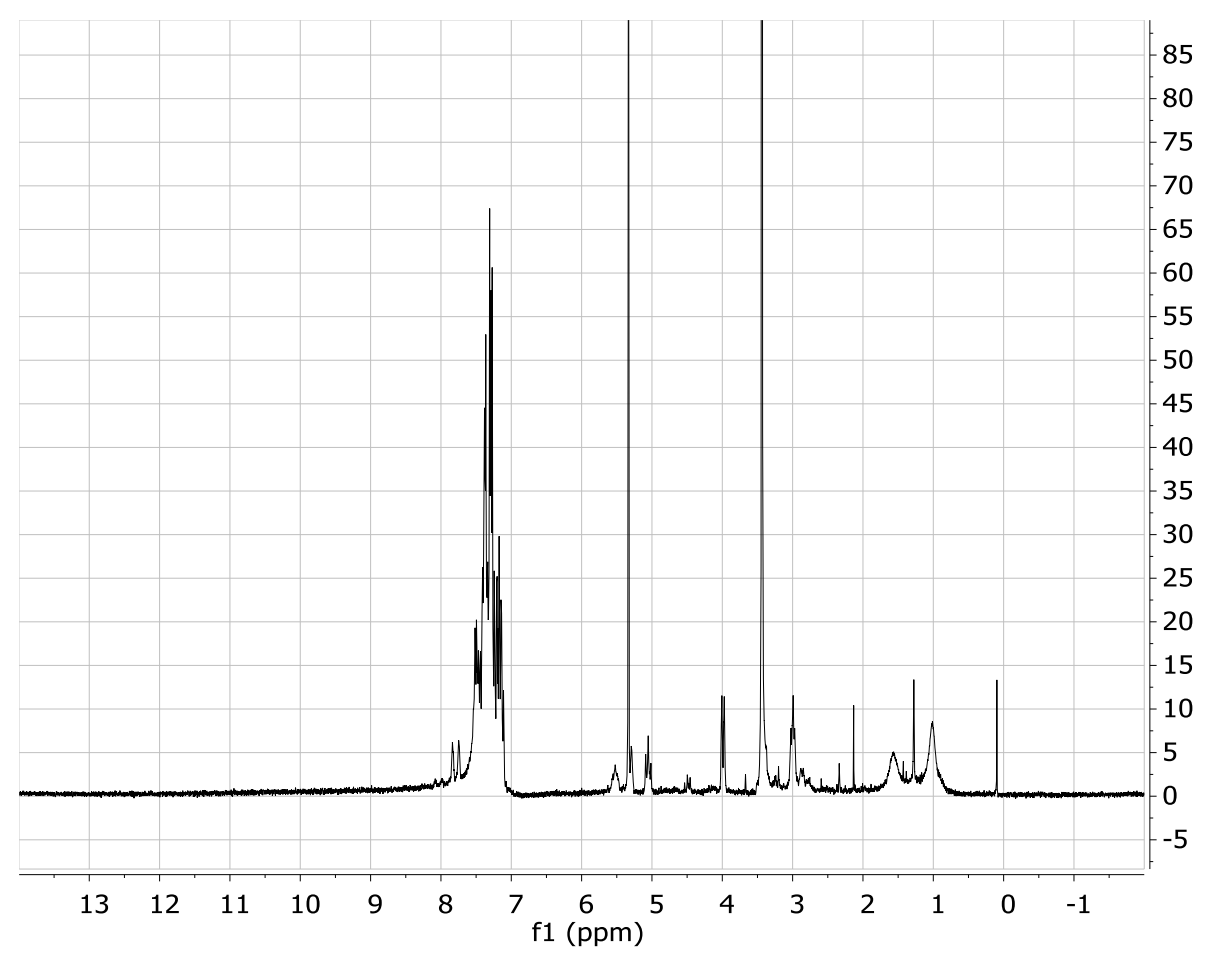

Figure S2 ${ }^{1} \mathrm{H}$ NMR $\left(300 \mathrm{MHz}, \mathrm{CD}_{2} \mathrm{Cl}_{2}\right)$ spectrum of $(S, S)-1 \mathrm{P} R_{2}=\mathrm{PEt}_{2}$ 


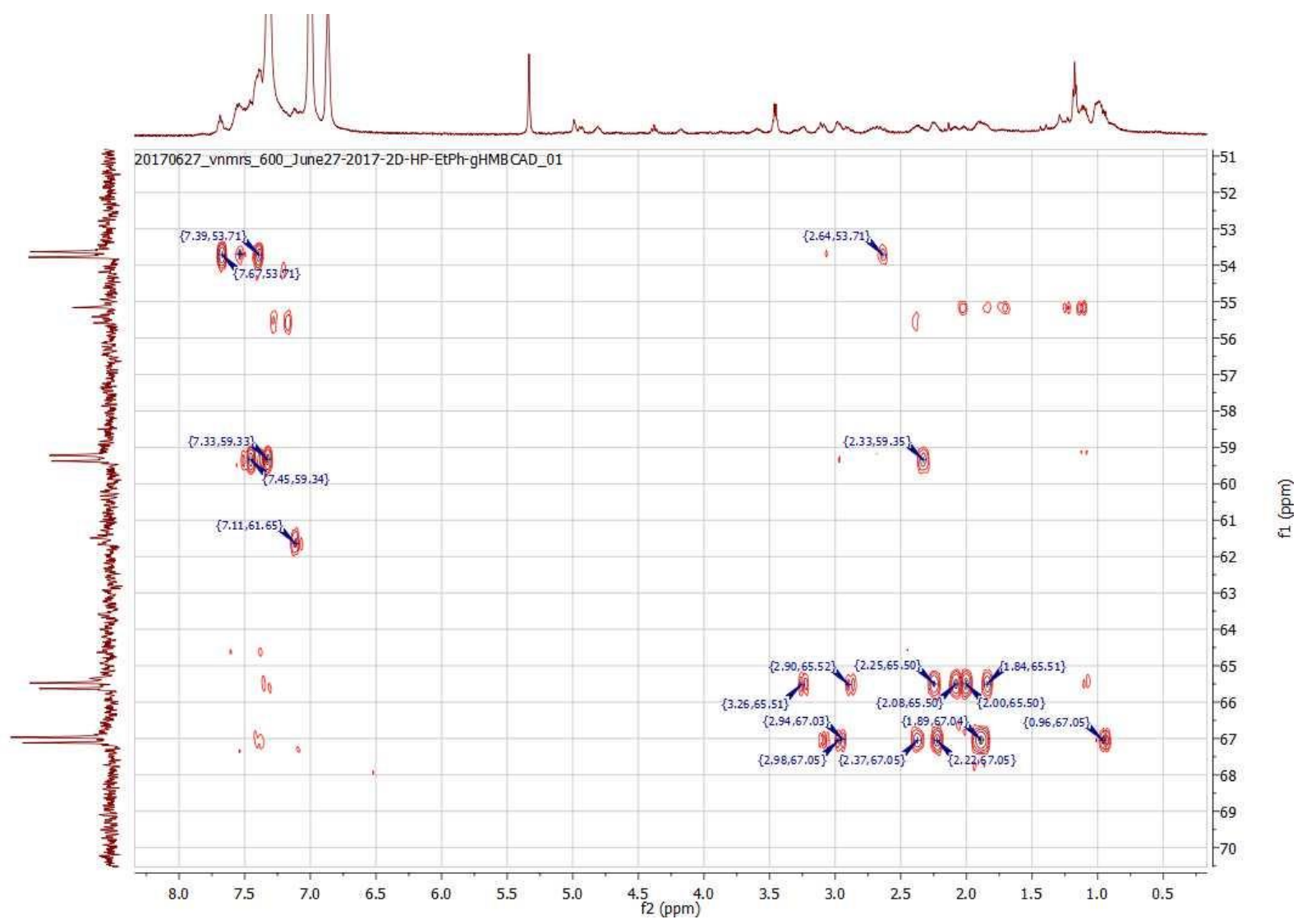

Figure S3. ${ }^{1} \mathrm{H} /{ }^{31} \mathrm{P}$ gHBMC spectrum $\left(600 \mathrm{MHz}, \mathrm{CD}_{2} \mathrm{Cl}_{2}\right)$ of $(S, S)-1 \mathrm{P} R_{2}=\mathrm{PEt}_{2}$. 


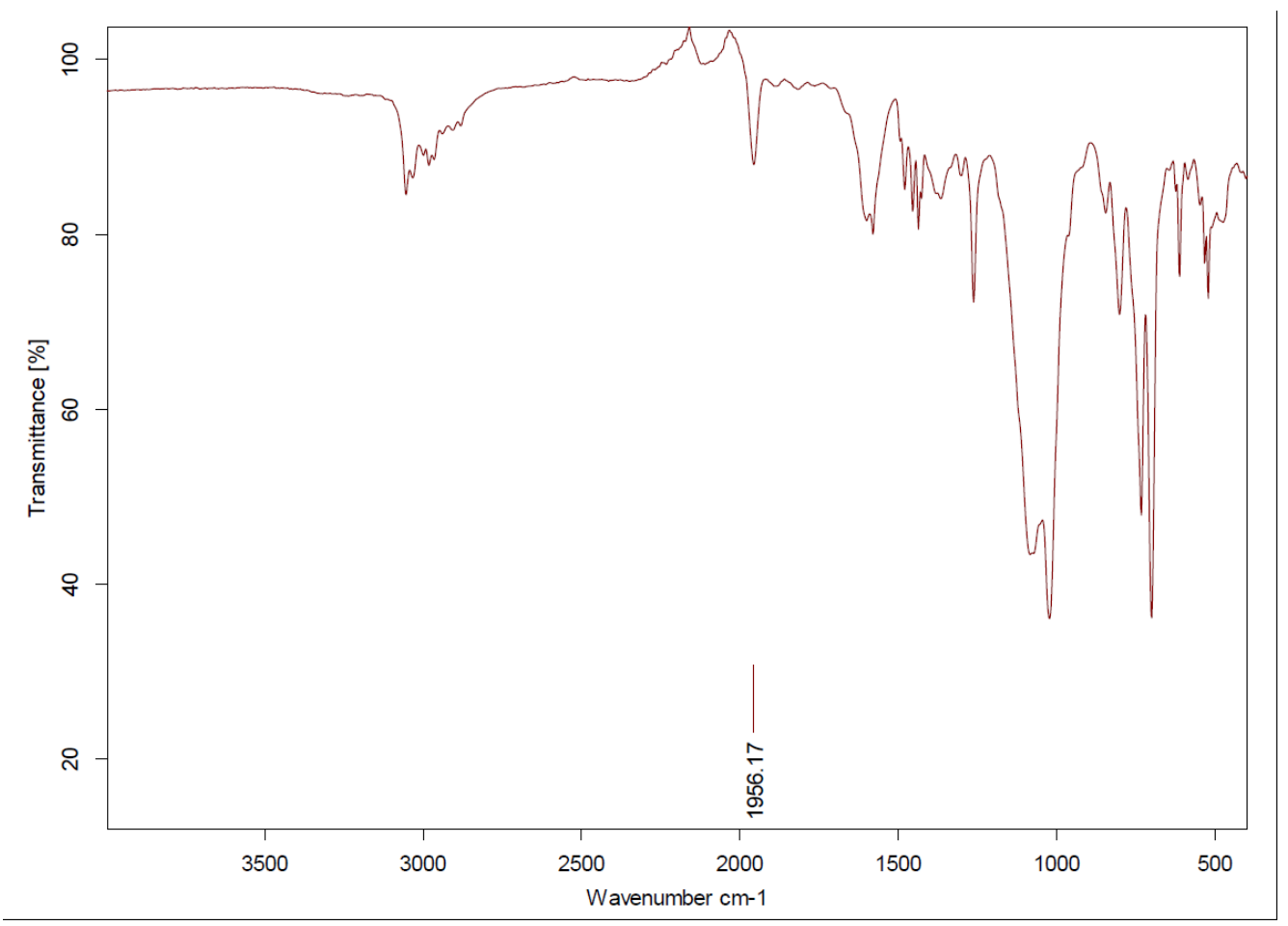

Figure S4 The FT-IR spectrum ( $\mathrm{KBr}$ pellet) of $(S, S)-1 \mathrm{P} R_{2}=\mathrm{PEt} t_{2}$. The peak at $1956 \mathrm{~cm}^{-1}$ was assigned to the $\mathrm{CO}$ stretch.

$(\mathrm{S}, \mathrm{S})-\left[\mathrm{FeBr}(\mathrm{CO})\left(\mathrm{PPh}_{2} \mathrm{CH}_{2} \mathrm{CH}_{2} \mathrm{NHCHPhCHPhN}=\mathrm{CHCH}_{2} \mathrm{P}(\mathrm{O}-\mathrm{Tol})_{2}\right)\right][\mathrm{BPh} 4] \quad((\mathrm{S}, \mathrm{S})-2)$. The procedure follows that of $(S, S)-1$. Yield: $90 \mathrm{mg}(56 \%)$. FT-IR $\left(\mathrm{KBr}, \mathrm{cm}^{-1}\right): 1963\left(\mathrm{v}_{\mathrm{co}}, w_{1 / 2} 20 \mathrm{~cm}^{-1}\right)$. HRMS (ESI-TOF, $\mathrm{CH}_{2} \mathrm{Cl}_{2}$ ) m/z calculated for $\left[\mathrm{C}_{45} \mathrm{H}_{45} \mathrm{BrFeN}_{2} \mathrm{OP}_{2}\right]^{+}:$825.1454, found: 825.1459. ${ }^{1} \mathrm{H} \mathrm{NMR}$ (300 MHz; $\left.\mathrm{CD}_{2} \mathrm{Cl}_{2}\right)$ \&: $1.27\left(\mathrm{~s}, 6 \mathrm{H}, \mathrm{P}\left(\mathrm{C}_{6} \mathrm{H}_{4}\left(\mathrm{CH}_{3}\right)\right)_{2}\right), 2.40\left(\mathrm{~m}, 2 \mathrm{H}, \mathrm{PCH}_{2}\right) 3.70\left(\mathrm{~m}, 2 \mathrm{H}, \mathrm{CH}_{2} \mathrm{CH}=\mathrm{N}\right), 3.89\left(\mathrm{~m}, 2 \mathrm{H}, \mathrm{NCH}_{2}\right)$, $5.04\left(\mathrm{~m}, 1 \mathrm{H}, \mathrm{NCHPh}\right.$; the other $\mathrm{CHPh}$ must overlap with $\left.\mathrm{CDHCl}_{2}\right), 6.5-7.60(\mathrm{~m}, 48 \mathrm{H}, \mathrm{ArH}), 7.6-7.8(\mathrm{~m}$, $1 \mathrm{H}, \mathrm{N}=\mathrm{CH}) .{ }^{31} \mathrm{P}\left\{{ }^{1} \mathrm{H}\right\} \mathrm{NMR}\left(121 \mathrm{MHz} ; \mathrm{CD}_{2} \mathrm{Cl}_{2}\right) \delta: 68.82\left(\mathrm{~d}, \mathrm{JPP}_{\mathrm{PP}}=36.7 \mathrm{~Hz}\right), 63.70\left(\mathrm{~d}, \mathrm{JPP}_{\mathrm{PP}}=38.8 \mathrm{~Hz}\right), 61.58(\mathrm{~d}$, $\left.J_{\mathrm{PP}}=41.4 \mathrm{~Hz}\right), 55.37\left(\mathrm{~d}, J_{\mathrm{PP}}=41.4 \mathrm{~Hz}\right), 54.13\left(\mathrm{~d}, J_{\mathrm{PP}}=38.8 \mathrm{~Hz}\right)$. EA [ $\left.\mathrm{C}_{69} \mathrm{H}_{64} \mathrm{BBrFeN}_{2} \mathrm{OP}_{2}\right]$ Expected: $\mathrm{C}$ 72.33\%, H 5.63\%, N 2.44\%. Analysis: C 72.61\%, H 6.24\%, N 2.57\%. 


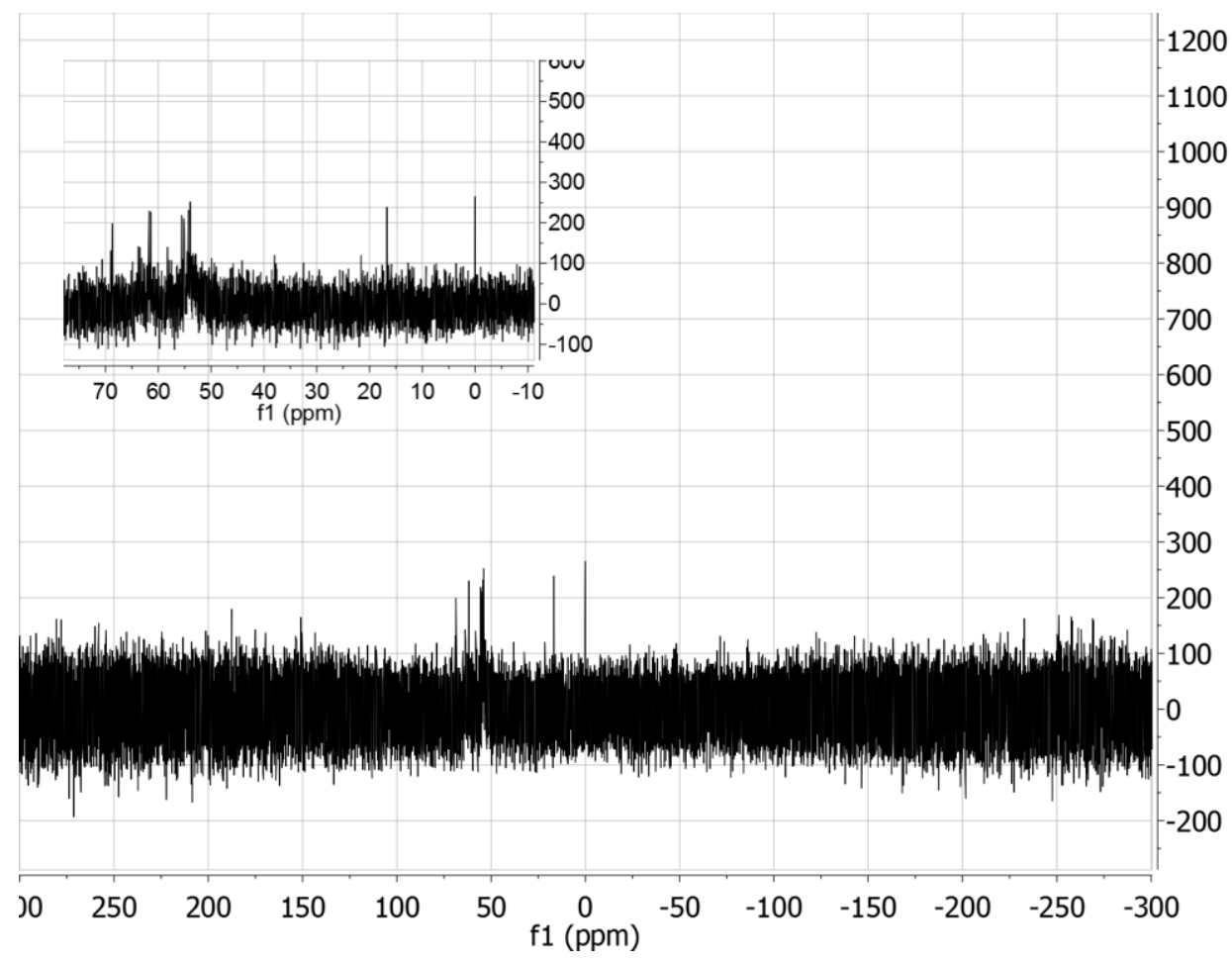

Figure S9 ${ }^{31} \mathrm{P}\left\{{ }^{1} \mathrm{H}\right\} \mathrm{NMR}\left(121 \mathrm{MHz}, \mathrm{CD}_{2} \mathrm{Cl}_{2}\right)$ spectrum of $(\mathrm{S}, \mathrm{S})-2 \mathrm{P} R_{2}=\mathrm{PoTol}_{2}$

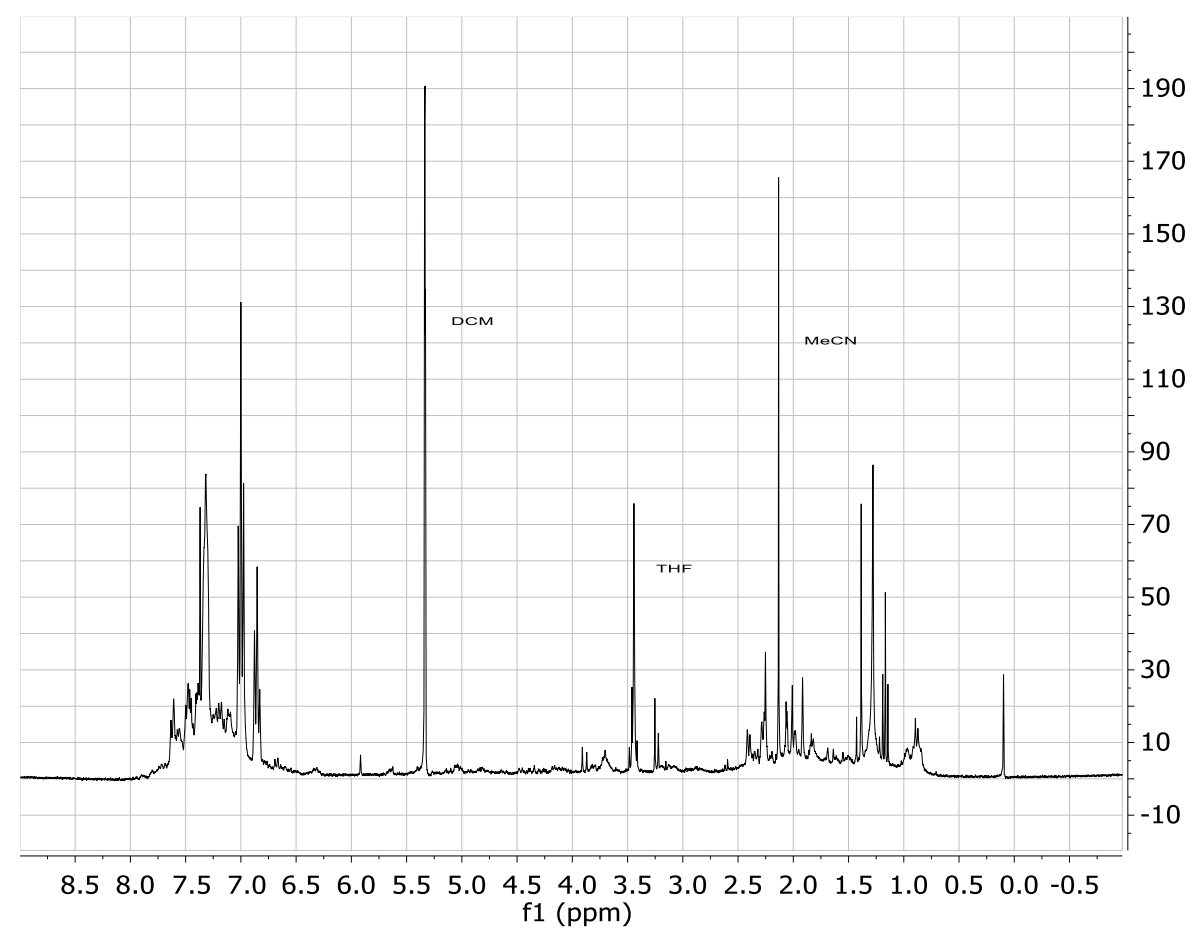

Figure $\mathrm{S} 10{ }^{1} \mathrm{H}$ NMR $\left(300 \mathrm{MHz}, \mathrm{CD}_{2} \mathrm{Cl}_{2}\right)$ spectrum of $(S, S)-2 \mathrm{P} R_{2}=\mathrm{PoTol}_{2}$ 


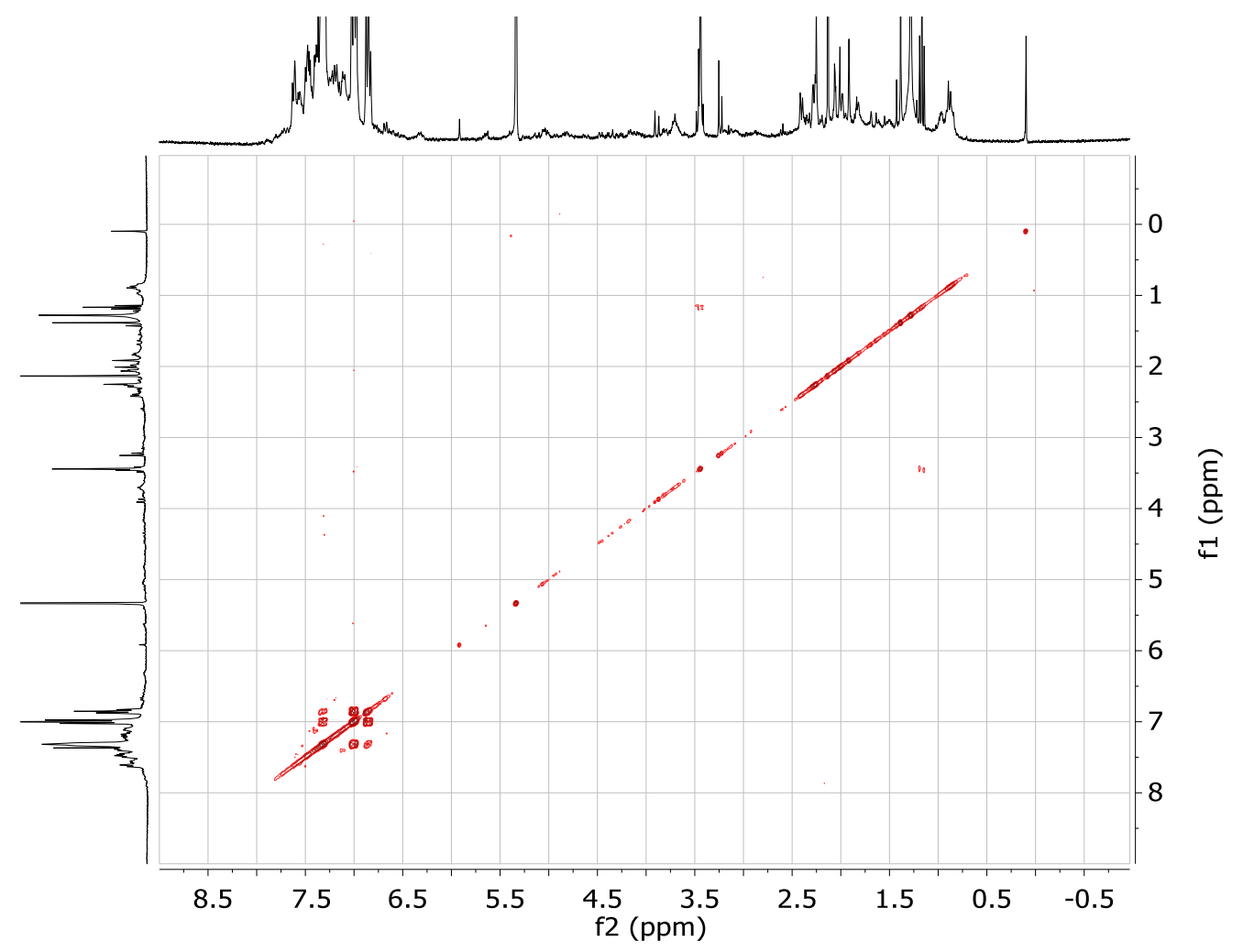

Figure S11 H-H COSY NMR (300 MHz, CD $\left.2 \mathrm{Cl}_{2}\right)$ spectrum of $(S, S)-2 \mathrm{P} R_{2}=\mathrm{PoTol}_{2}$

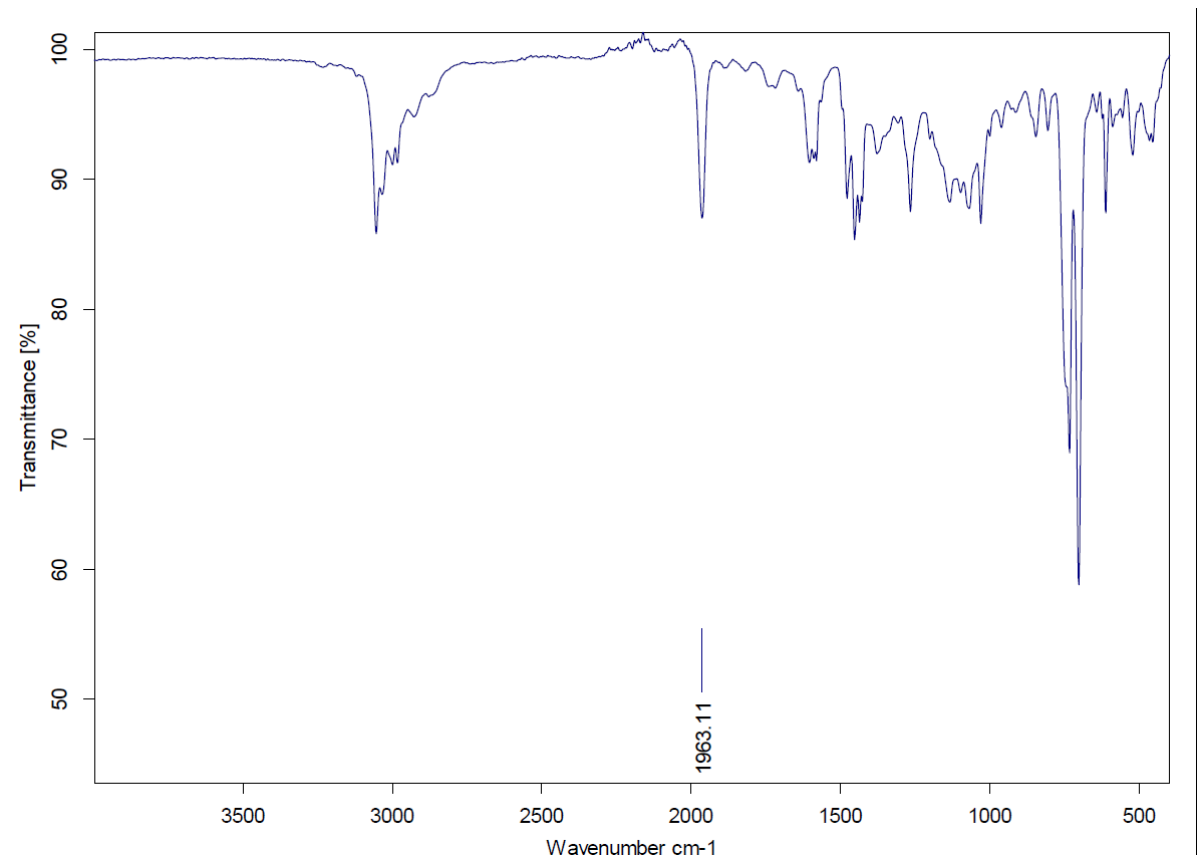

Figure S12 The FT-IR spectrum ( $\mathrm{KBr}$ pellet) of $(S, S)-2 \mathrm{P} R_{2}=$ PoTol $_{2}$. The peak at $1963 \mathrm{~cm}^{-1}$ was assigned to the $\mathrm{CO}$ stretch. 


\section{Observation by NMR of the Iron amido-eneamido and hydride}

\section{$\mathrm{Fe}(\mathrm{H})(\mathrm{CO})\left(\mathrm{PPh}_{2} \mathrm{CH}_{2} \mathrm{CH}_{2} \mathrm{NHCHPhCHPhNCH}=\mathrm{CHPCY}_{2}\right)(\mathrm{S}, \mathrm{S})-3$. Amido-eneamido: (S, S)-1 (20}

$\mathrm{mg}, 1.96 \times 10^{-5} \mathrm{~mol}$ ) and KOtBu (8 eq., $18 \mathrm{mg}$ ) were stirred in THF for $5 \mathrm{~min}$, then dried in vacuo. The residue was dissolved in $\mathrm{C}_{6} \mathrm{D}_{6}$ and filtered through a Syringe Filter PTFE membrane (pore size 0.45 $\mu \mathrm{m})$. Two compounds were present in NMR. Compound $1:{ }^{1} \mathrm{H}$ NMR $\left(400 \mathrm{MHz} ; \mathrm{C}_{6} \mathrm{D}_{6}\right) \delta: 3.32(\mathrm{t}, 1 \mathrm{H}$, $\left.\mathrm{PCH}, J_{\mathrm{HH}}=4.7 \mathrm{~Hz}\right), 4.58\left(\mathrm{t}, 1 \mathrm{H}, \mathrm{C}(\mathrm{H})(\mathrm{Ph}), J_{\mathrm{HH}}=8.7 \mathrm{~Hz}\right), 5.36\left(\mathrm{~d}, 1 \mathrm{H}, \mathrm{C}(\mathrm{H})(\mathrm{Ph}), J_{\mathrm{HH}}=10.3 \mathrm{~Hz}\right), 7.65(\mathrm{t}, 1 \mathrm{H}$, $\left.\mathrm{NCH}=\mathrm{CH}, J_{\mathrm{HH}}=8.7 \mathrm{~Hz}\right) .{ }^{31} \mathrm{P}\left\{{ }^{1} \mathrm{H}\right\} \mathrm{NMR}\left(161 \mathrm{MHz} ; \mathrm{C}_{6} \mathrm{D}_{6}\right) \delta: 86.30\left(\mathrm{~d}, J_{\mathrm{PP}}=25.2 \mathrm{~Hz}\right), 82.68\left(\mathrm{~d}, J_{\mathrm{PP}}=25.2 \mathrm{~Hz}\right)$; Compound 2: ${ }^{1} \mathrm{H}$ NMR $\left(400 \mathrm{MHz} ; \mathrm{C}_{6} \mathrm{D}_{6}\right) \delta: 3.44\left(\mathrm{dd}, 1 \mathrm{H}, \mathrm{PCH}, \mathrm{J}_{\mathrm{HH}}=5.0,3.0 \mathrm{~Hz}\right), 4.40(\mathrm{~d}, 1 \mathrm{H}, \mathrm{C}(H)(\mathrm{Ph})$, $\left.J_{\mathrm{HH}}=3.0 \mathrm{~Hz}\right), 4.73\left(\mathrm{~d}, 1 \mathrm{H}, \mathrm{C}(H)(\mathrm{Ph}), J_{\mathrm{HH}}=3.5 \mathrm{~Hz}\right), 7.62(\mathrm{~m}, 1 \mathrm{H}, \mathrm{NCH}=\mathrm{CH}) .{ }^{31} \mathrm{P}\left\{{ }^{1} \mathrm{H}\right\} \mathrm{NMR}\left(161 \mathrm{MHz} ; \mathrm{C}_{6} \mathrm{D}_{6}\right)$ $\delta: 91.42\left(\mathrm{~d}, J_{\mathrm{PP}}=27.5 \mathrm{~Hz}\right), 82.71\left(\mathrm{~d}, J_{\mathrm{PP}}=27.5 \mathrm{~Hz}\right)$.

Hydride (S,S)-3: The solution from above was dried then dissolved in $\mathrm{PrOH}$ and stirred for 2 mins. This solution was dried overnight. FT-IR $\left(\mathrm{KBr}, \mathrm{cm}^{-1}\right)$ : $1894\left(\mathrm{v}_{\mathrm{co}}\right) .{ }^{1} \mathrm{H} \mathrm{NMR}\left(400 \mathrm{MHz} ; \mathrm{C}_{6} \mathrm{D}_{6}\right) \delta:-10.27$ (dd, $\left.1 \mathrm{H}, \mathrm{FeH}, \mathrm{J}_{\mathrm{HP}}=82.2,59.8\right) .{ }^{31} \mathrm{P}\left\{{ }^{1} \mathrm{H}\right\} \mathrm{NMR}\left(161 \mathrm{MHz} ; \mathrm{C}_{6} \mathrm{D}_{6}\right) \delta: 91.19\left(\mathrm{~d}, \mathrm{~J}_{\mathrm{PP}}=36.6 \mathrm{~Hz}\right), 83.02\left(\mathrm{~d}, \mathrm{~J}_{\mathrm{PP}}=\right.$ $36.6 \mathrm{~Hz})$.

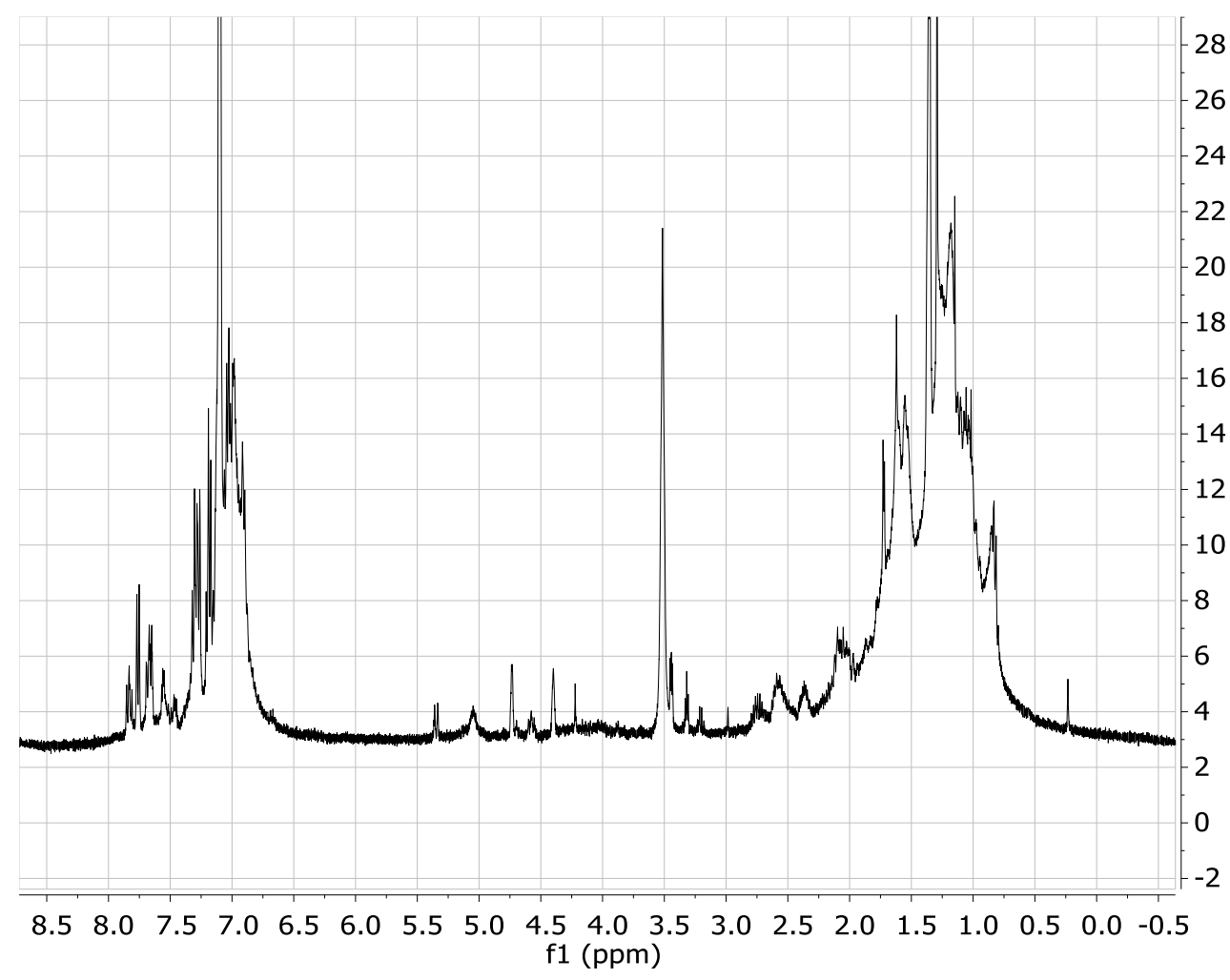

Figure $\mathrm{S} 16{ }^{1} \mathrm{H} N M R\left(400 \mathrm{MHz}, \mathrm{C}_{6} \mathrm{D}_{6}\right)$ spectrum of Amido-eneamido $\mathrm{P} R_{2}=\mathrm{PC} y_{2}$ 


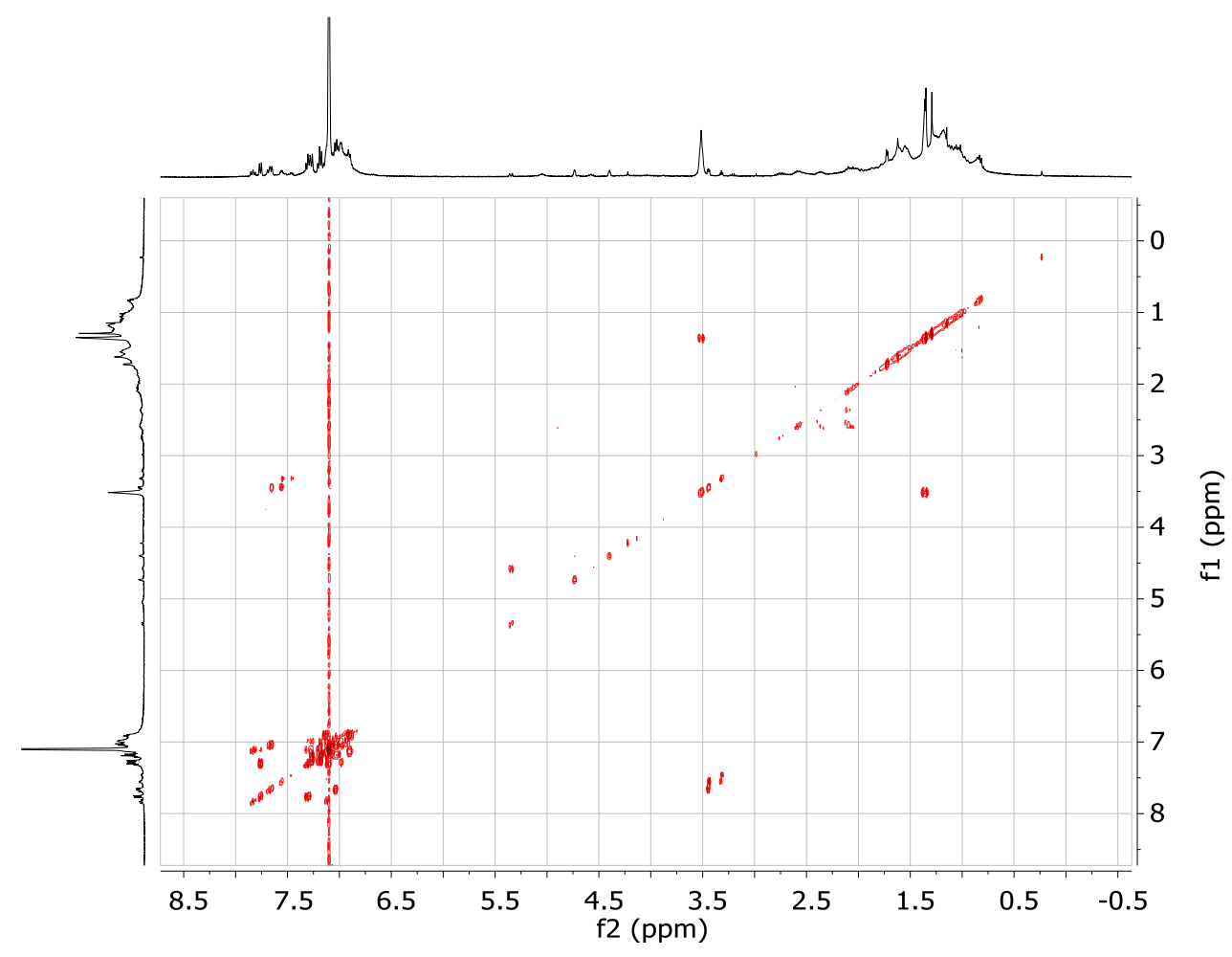

Figure $\mathrm{S} 17{ }^{1} \mathrm{H}^{-1} \mathrm{H}$ COSY NMR (400 MHz, $\mathrm{C}_{6} \mathrm{D}_{6}$ ) spectrum of Amido-eneamido $\mathrm{P} R_{2}=\mathrm{PCy}$

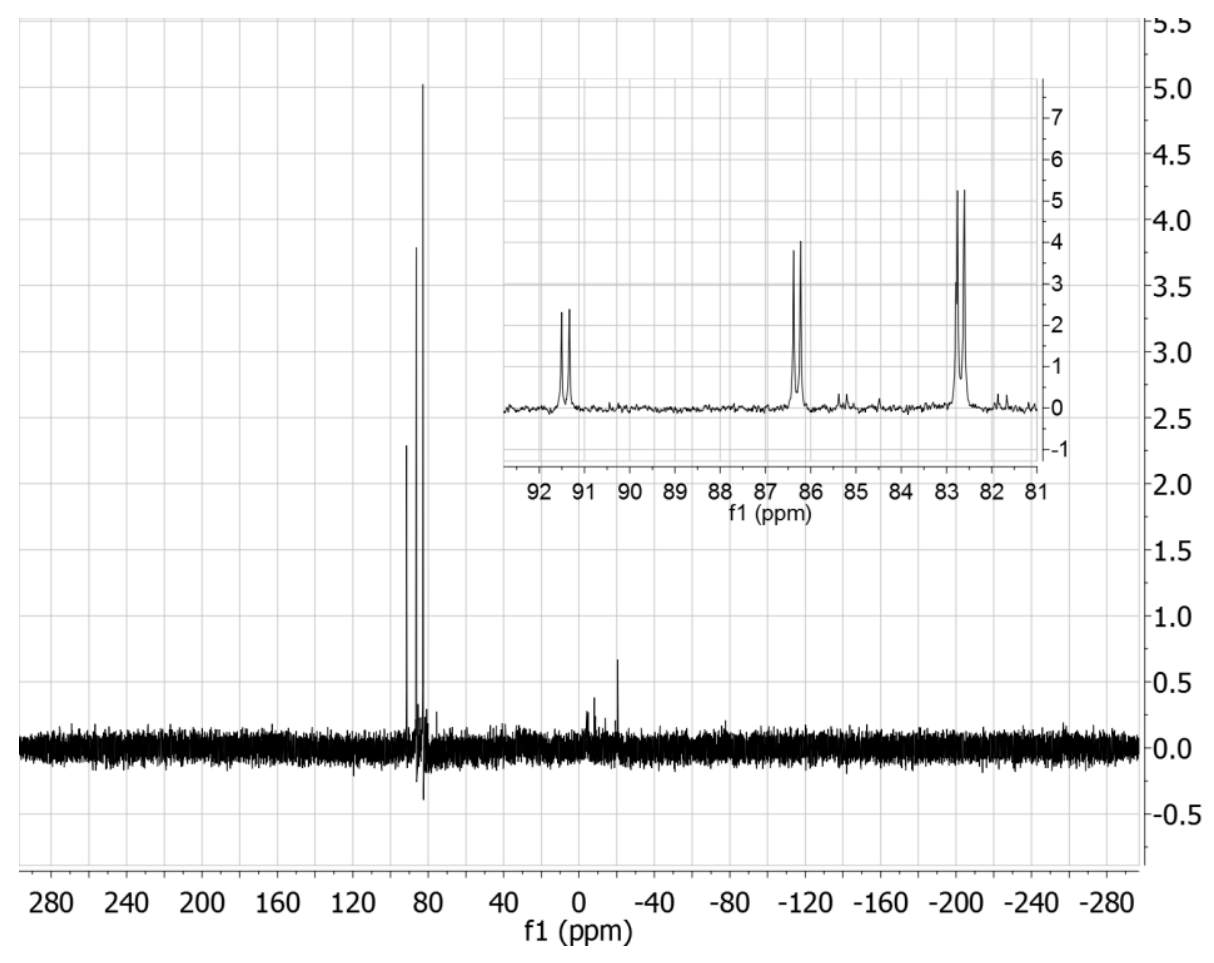

Figure $\mathrm{S} 18^{31} \mathrm{P}\left\{{ }^{1} \mathrm{H}\right\}$ NMR (161 MHz, $\left.\mathrm{C}_{6} \mathrm{D}_{6}\right)$ spectrum of Amido-eneamido $\mathrm{P} R_{2}=\mathrm{PC} y_{2}$ 


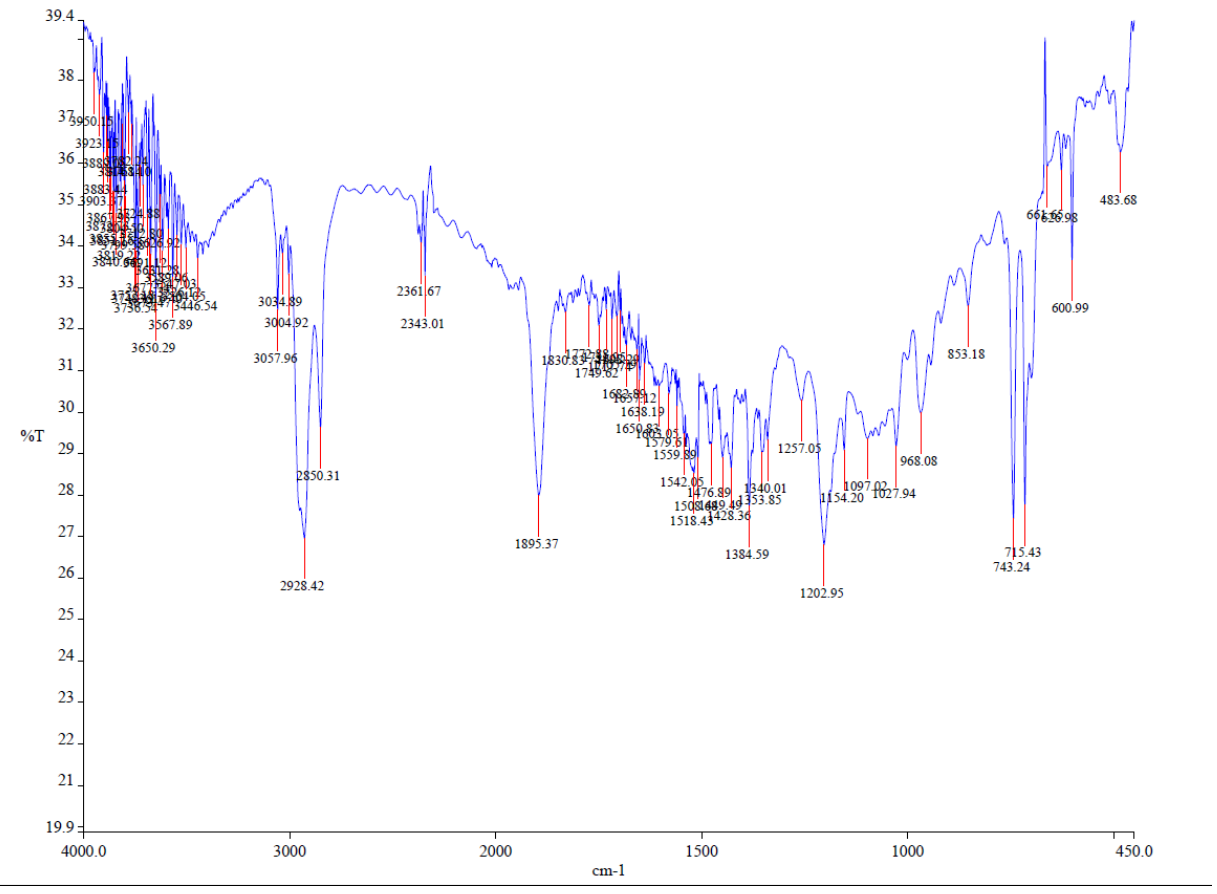

Figure S19 The FT-IR spectrum ( $\mathrm{KBr}$ pellet) of Amido-eneamido $\mathrm{P} R_{2}=\mathrm{PCy}$. The peak at $1895 \mathrm{~cm}^{-1}$ was assigned to the $\mathrm{CO}$ stretch

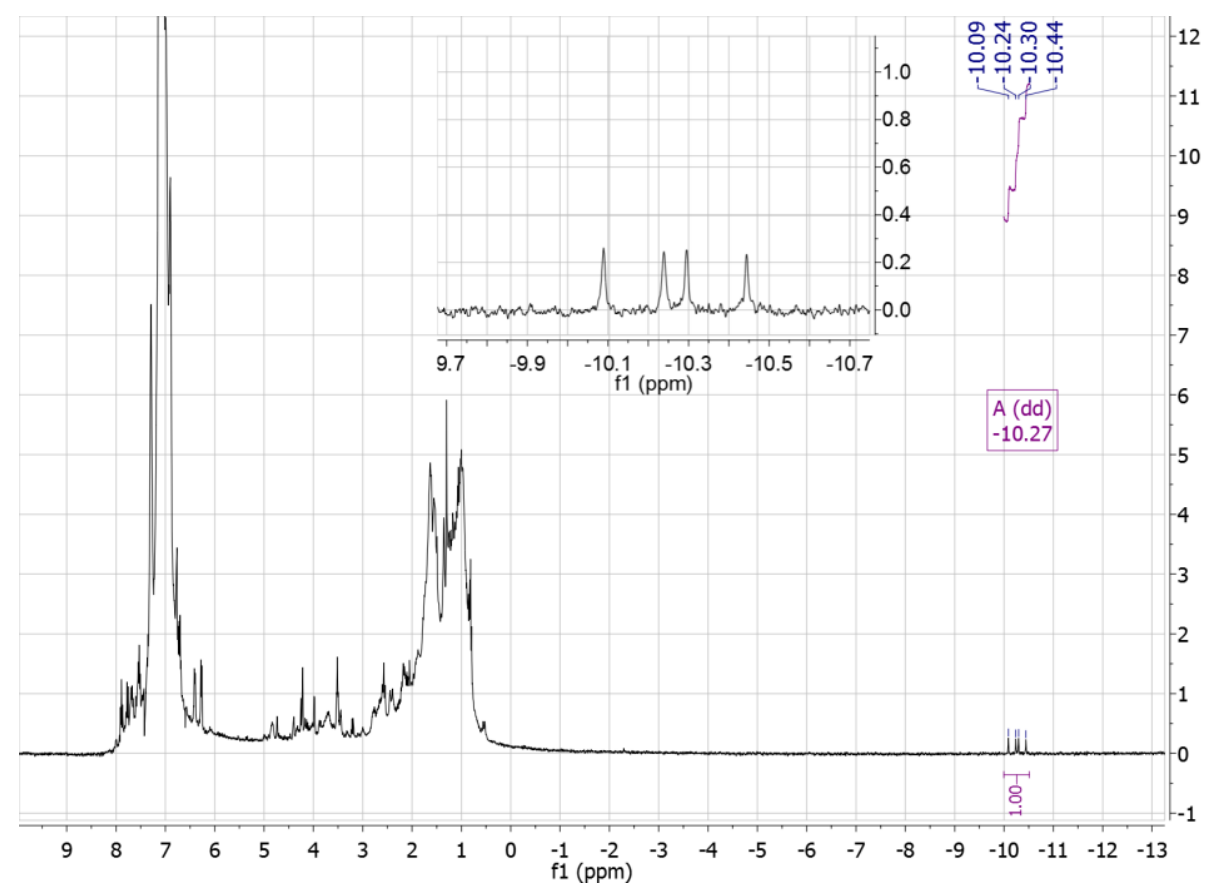

Figure $S 20{ }^{1} \mathrm{H}$ NMR $\left(400 \mathrm{MHz}, \mathrm{C}_{6} \mathrm{D}_{6}\right)$ spectrum of Hydride $(S, S)-3 \mathrm{P} R_{2}=\mathrm{PC} y_{2}$ 


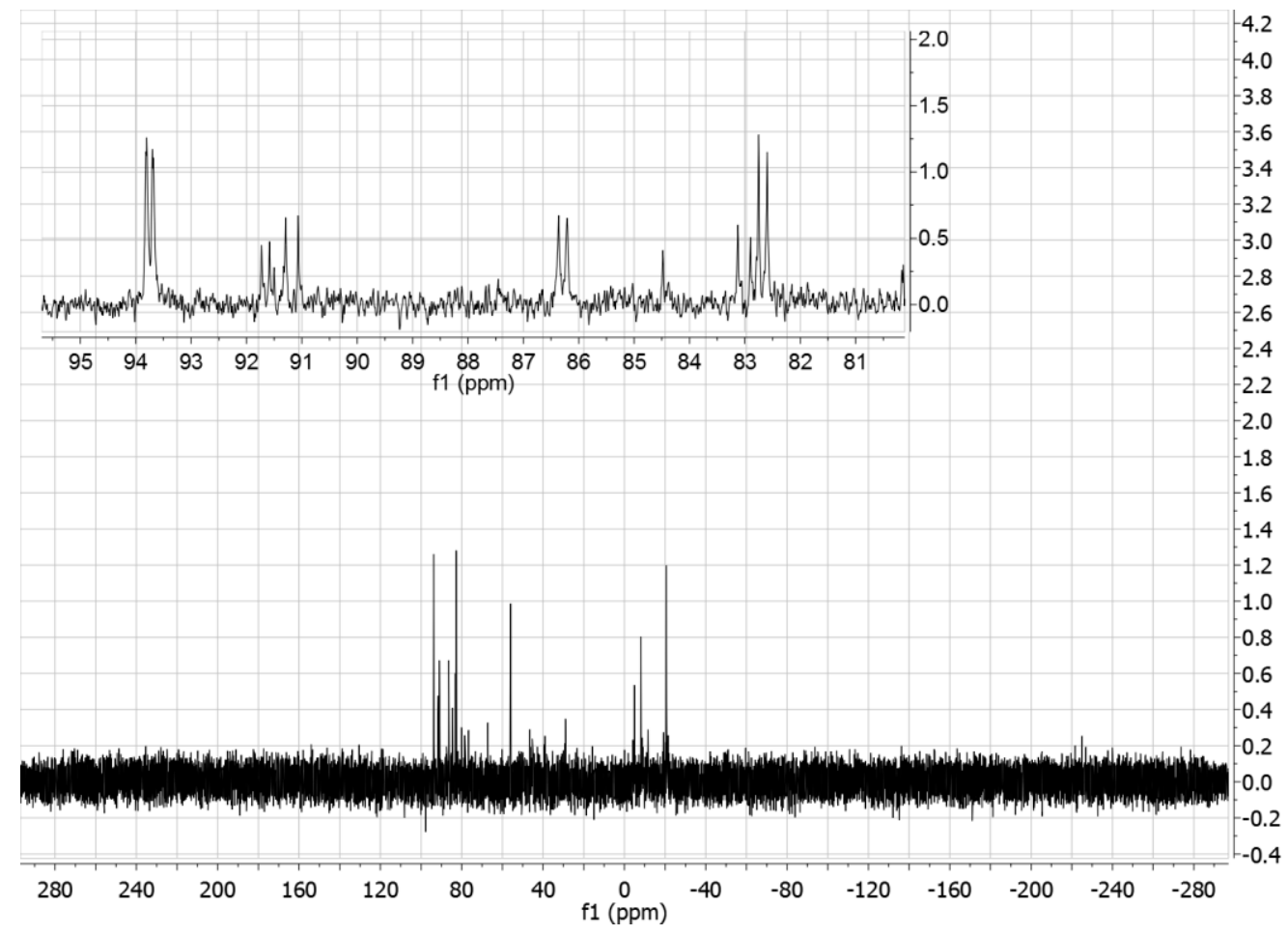

Figure S21 ${ }^{31} \mathrm{P}\left\{{ }^{1} \mathrm{H}\right\} \mathrm{NMR}\left(161 \mathrm{MHz}, \mathrm{C}_{6} \mathrm{D}_{6}\right)$ spectrum of Hydride $(S, S)-3 \mathrm{P} R_{2}=\mathrm{PC} y_{2}$

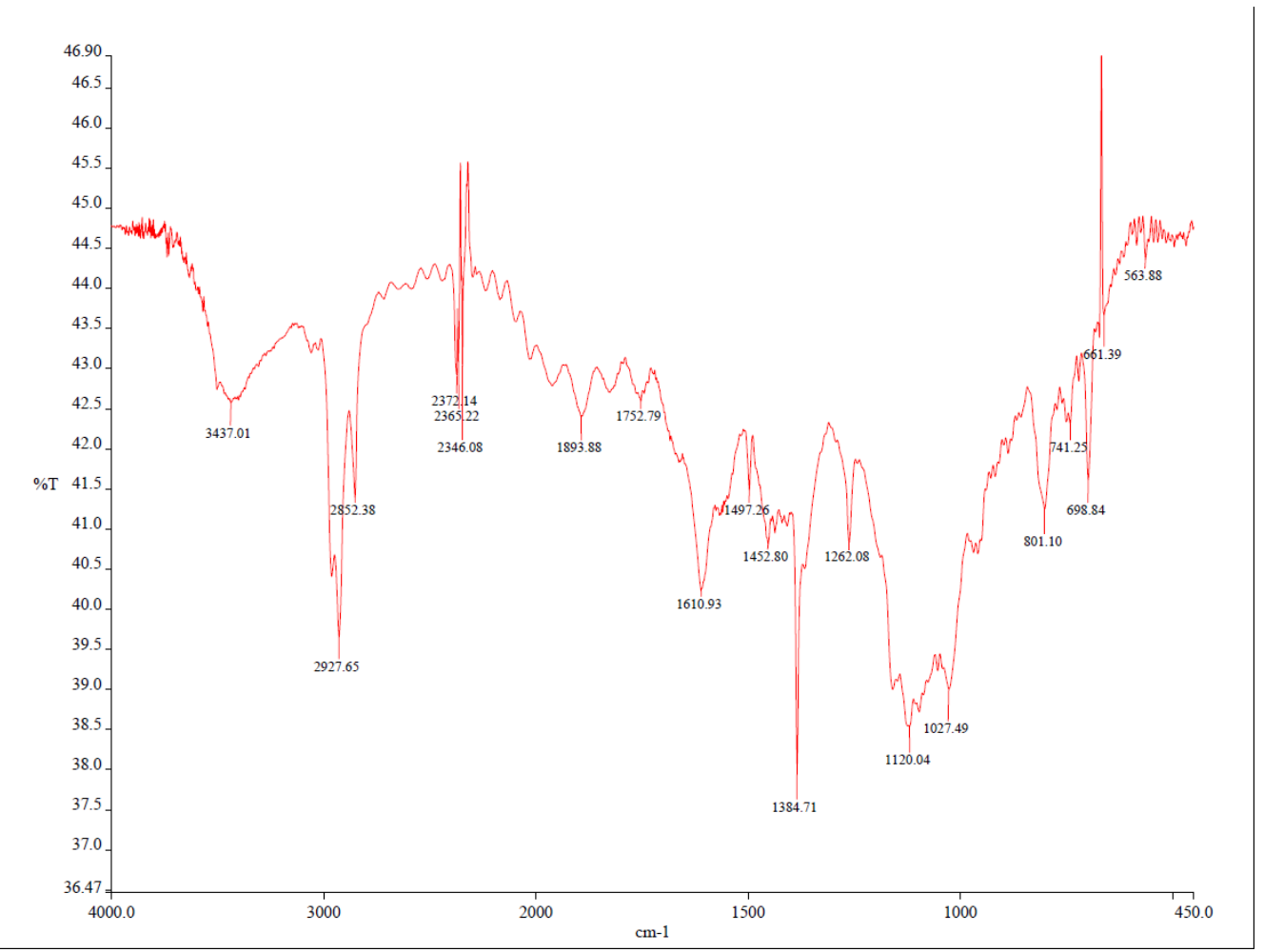

Figure S22 The FT-IR spectrum (KBr pellet) of Hydride $(S, S)-3 P R_{2}=P C y_{2}$. The peak at $1894 \mathrm{~cm}^{-1}$ was assigned to the $\mathrm{CO}$ stretch 


\section{General procedure for ATH (C/B/S=1/2/500):}

A $20 \mathrm{~mL}$ vial was charged with a stir bar and precatalyst $\left(8.9 \times 10^{-3} \mathrm{mmol}\right)$. The substrate $(4.43 \times 10 \mathrm{mmol})$ was added and the mixture was stirred. For liquid substrates, the mixture was stirred until the catalyst was completely dissolved. $3.61 \mathrm{~g}$ 'PrOH was added and the solution was stirred for 5 minutes, or longer for solid substrates to allow them to fully dissolve. A stock solution of KOtBu ( $20 \mathrm{mg}, 0.18 \mathrm{mmol})$ in $0.98 \mathrm{~g}$ ' $\mathrm{PrOH}$ was stirred until all the base was dissolved. $0.1 \mathrm{~g}$ stock solution (2 eq.) was diluted by 1.0 $\mathrm{g}$ 'PrOH and added to the $20-\mathrm{mL}$ vial to activate the precatalyst and start catalysis. $0.1 \mathrm{~mL}$ samples were taken via syringe and injected into Teflon-sealed GC vials prepared with wet, aerated ${ }^{2} \mathrm{PrOH}$ to quench catalysis.

\section{General procedure for ATH (C/B/S=1/8/6121):}

The quantity of the precatalyst was measured via a stock solution method. A concentrated stock solution was made by dissolving the precatalysts $\left(1.97 \times 10^{-2} \mathrm{mmol}\right)$ in $6.08 \mathrm{~g}$ cold dichloromethane. After all the solid was dissolved, the solution was immediately sucked into a syringe. The solution was then divided into equal portions into several $20 \mathrm{~mL}$ vials such that each portion has $0.2 \mathrm{~g}$ of the stock solution, and then dichloromethane was removed under vacuum. These operations led to a precatalyst quantity of $6.48 \times 10^{-4} \mathrm{mmol}$ in each vial. The base was prepared by dissolving KOtBu (10 $\mathrm{mg}, 0.089$ $\mathrm{mmol})$ in ${ }^{\mathrm{P}} \mathrm{POH}(1.02 \mathrm{~g}, 1.30 \mathrm{~mL}){ }^{\mathrm{i}} \mathrm{PrOH}(6.63 \mathrm{~g}, 8.44 \mathrm{~mL})$, substrate $(3.95 \mathrm{mmol})$ and a clean stirring bar were added to the vial that contains the precatalyst and the solution was stirred for 5 minutes, or until it was dissolved. $0.015 \mathrm{~g}$ of the base stock solution was added into a vial that contains $0.546 \mathrm{~g}$ of iPrOH and the mixed solution was then added into the catalyst solution to start the catalytic reaction. $0.1 \mathrm{~mL}$ samples were taken via syringe and injected into Teflon-sealed GC vials prepared with wet, aerated 'PrOH to quench catalysis.

\section{Data for analysis of the structures of the isomers of complexes} $\left[\mathrm{Fe}(\mathrm{CO})(\mathrm{Br})\left(\mathrm{PR}_{2} \mathrm{CH}_{2} \mathrm{CHNCHPhCHPhNHCH}{ }_{2} \mathrm{CH}_{2} \mathrm{PPh}_{2}\right)\right]^{+}$

Position of the NH relative to the chiral centre on dpen. All of the structures of metal derivatives of racemic or enantiopure dpen in the Cambridge Crystallographic Data Bank containing the structure $\mathrm{C}-\mathrm{NH}-\mathrm{CHPh}$ - have the $\mathrm{NH}$ and $\mathrm{CH}$ hydrogens positioned anti to each other with the phenyl group equatorial in the five member ring. See the structures with refcodes ABALAR, BEPGAF, BOXVOA, CAGLUT, CAGMAA, DAQDIJ, DAQDOP, DOZMAH, GASGUD, IJIHIS, KUPDIJ, KUPDOP, KUYHET, LIPDUL, LIPFAT, MIMKAW, NIDXEE, ONOXOG, ONOXUM, OPOSOC, OPOSUI, QENJEY, QOPHAG, RAZNUD, RAZPAL, RAZPEP, RILYES, SITSEU, SOJTUG, TANROQ, TEJLAX, TEJLEB, TIXNAQ, TIXNEU, TIXNIY, TUSPOO, VEDPUQ, XOSCEP, YOSXEJ, YUFJOZ, YUFJUF, ZOWNIK, TAQJIH.

\section{Analysis of the NMR spectra of the trans isomers}

The complexes $\mathbf{C} \mathbf{1}$ to $\mathbf{C} \mathbf{3}$ are thought to have the trans-configuration with structure E in Figure S23. 

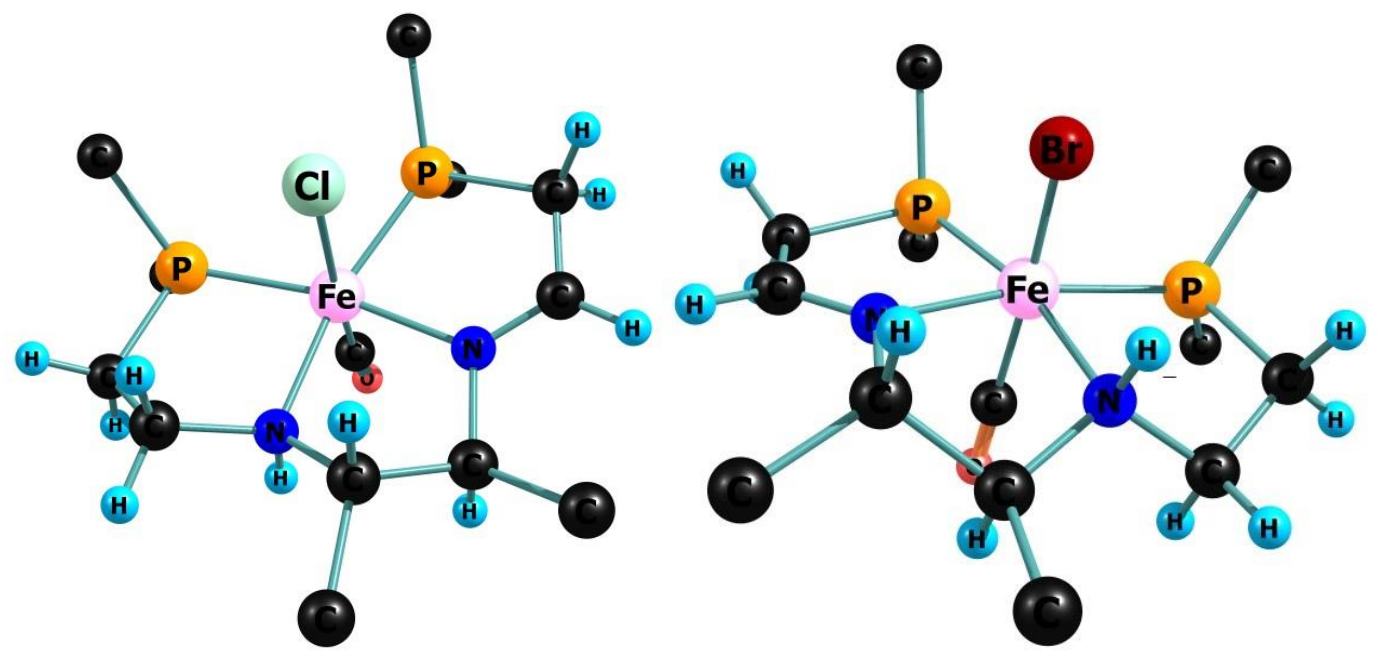

Figure S23. Structure E of C3 (left) and proposed structure E' of C4 in solution.

${ }^{31} \mathrm{P} N M R$

Analysis of the $1 \mathrm{H}$ NMR spectra of the backbone regions of $\mathrm{C} 1, \mathrm{C} 4-1$ and $(\mathrm{S}, \mathrm{S})-1$ in $\mathrm{CD}_{2} \mathrm{Cl}_{2}$ solution.

2D NMR spectra and spin simulation software MNova-11 were used to assign the proton spectra between 2 and $6 \mathrm{ppm}$ where the signals of the backbone of the tetradentate ligand are found apart from the imine $\mathrm{CH}=\mathrm{N}$ proton which is hidden under the aryl proton resonances. The results are found in Table S1 and the simulated spectra in Figures S24-S27.

Table S1. Chemical shifts and coupling constants for the ${ }^{1} \mathrm{H}$ NMR of backbone resonances of $\mathbf{C 1}, \mathbf{C 4}-\mathbf{1}$ and $(\mathbf{S}, \mathbf{S})-\mathbf{1}$ in $\mathrm{CD}_{2} \mathrm{Cl}_{2}$ solution; refer to the structures with the spectra for the lettering of hydrogen and phosphorus atoms.

\begin{tabular}{|c|c|c|c|c|c|c|c|c|c|c|c|c|c|c|c|c|c|c|c|}
\hline \multirow[b]{2}{*}{ PR2 } & \multirow{2}{*}{\begin{tabular}{|l|} 
31P NMR \\
PPh2 \\
\end{tabular}} & \multicolumn{2}{|l|}{ 31P NMR } & & \multirow[b]{2}{*}{ H } & 1H NMR & \multirow[b]{2}{*}{ B } & \multirow[b]{2}{*}{ C } & & \multirow[b]{2}{*}{ D } & \multirow[b]{2}{*}{ E } & \multirow[b]{2}{*}{$\mathbf{F}$} & \multirow{2}{*}{\multicolumn{2}{|c|}{ G }} & & \multirow[b]{2}{*}{ A2 } & & \multirow[b]{2}{*}{ B2 } & \multirow[b]{2}{*}{ C2 } \\
\hline & & PR2 & JPP & & & A & & & & & & & & & & & & & \\
\hline & $\mathrm{ppm}$ & ppm & $\mathrm{Hz}$ & & & ppm & ppm & $\mathrm{ppm}$ & & ppm & ppm & $\mathrm{ppm}$ & & ppm & & ppm & & $\mathrm{ppm}$ & ppm \\
\hline PPh2, C1 & $\mathrm{CD} 2 \mathrm{Cl} 2$ & & & & & $\mathrm{~J}, \mathrm{~Hz}$ & $\mathrm{~J}, \mathrm{~Hz}$ & $\mathrm{~J}, \mathrm{~Hz}$ & & $\mathrm{~J}, \mathrm{~Hz}$ & $\mathrm{~J}, \mathrm{~Hz}$ & $\mathrm{~J}, \mathrm{~Hz}$ & & $\mathrm{~J}, \mathrm{~Hz}$ & & $\mathrm{~J}, \mathrm{~Hz}$ & & $\mathrm{~J}, \mathrm{~Hz}$ & $\mathrm{~J}, \mathrm{~Hz}$ \\
\hline \multirow[t]{6}{*}{ Isomer 1} & 57.9 & 62.7 & 40 & $\delta$ & & 3.00 & 2.84 & 2.99 & & 3.42 & 5.59 & 5.08 & & 5.32 & & 3.98 & & 4.00 & 7.84 \\
\hline & $\mathrm{H}$ & D2 & & JHH & $A B$ & $12 \mathrm{AB}$ & $12 \mathrm{AC}$ & & $A D$ & $12 \mathrm{DE}$ & $12 \mathrm{EF}$ & 10 & FG & 10 & A2B2 & 12 & A2B2 & 12 & \\
\hline & & & & JHH & $A C$ & $6 \mathrm{BC}$ & $2 \mathrm{BC}$ & & $B D$ & $7 \mathrm{EF}$ & $10 \mathrm{FG}$ & 10 & & & $\mathrm{~A} 2 \mathrm{C} 2$ & & B $22 \mathrm{C} 2$ & 4 & \\
\hline & & & & JHH & $A D$ & $12 \mathrm{BD}$ & $7 \mathrm{CD}$ & 12 & $C D$ & 12 & & & & & & & & & \\
\hline & & & & JHH & & & & & DE & 12 & & & & & & & & & \\
\hline & & & & JHP & $\mathrm{AH}$ & $6 \mathrm{BH}$ & 55 & 10 & $\mathrm{DH}$ & $10 \mathrm{EH}$ & $4 \mathrm{FH}$ & & $\mathrm{GH}$ & 4 & A2D2 & 10 & B2D2 & 10 & \\
\hline PEt2, $(S, S)-1$ & PPh2 & PR2 & JPP & & H & A & B & C & & D & E & $\mathbf{F}$ & & G & & A2 & & B2 & C2 \\
\hline \multirow[t]{6}{*}{ SS1-1 } & 59.3 & 65.5 & 37.9 & $\delta$ & & 2.34 & 3.10 & 2.97 & & 2.38 & 4.16 & 4.36 & & 4.92 & & 2.89 & & 3.09 & 7.33 \\
\hline & $\mathrm{H}$ & D2 & & JHH & $A B$ & $12 \mathrm{AB}$ & $12 \mathrm{AC}$ & & $A D$ & $14 \mathrm{CE}$ & $7 \mathrm{EF}$ & 10 & FG & 10 & A2B2 & 12.5 & $\mathrm{~A} 2 \mathrm{~B} 2$ & 12.5 & \\
\hline & & & & JHH & $A C$ & $7 \mathrm{BC}$ & $2 \mathrm{BC}$ & & $B D$ & $7 \mathrm{DE}$ & $13 \mathrm{FG}$ & 10 & & & $\mathrm{~A} 2 \mathrm{C} 2$ & & $\mathrm{~B} 2 \mathrm{C} 2$ & & \\
\hline & & & & JHH & $A D$ & $14 \mathrm{BD}$ & $7 \mathrm{CD}$ & 12 & $C D$ & $12 \mathrm{EF}$ & 10 & & & & & & & & \\
\hline & & & & JHH & & & CE & & DE & 13 & & & & & & & & & \\
\hline & & & & JHP & $\mathrm{AH}$ & $6 \mathrm{BH}$ & $22 \mathrm{CH}$ & 15 & $\mathrm{DH}$ & $10 \mathrm{EH}$ & $6 \mathrm{FH}$ & 0 & & & A2D2 & & B2D2 & 10 & \\
\hline PEt2, $(S, S)-1$ & PPh2 & PR2 & JPP & & H & A & B & C & & D & E & $\mathbf{F}$ & & G & & A2 & & B2 & C2 \\
\hline \multirow[t]{6}{*}{ SS1-2 } & 53.7 & 67.1 & 36.4 & $\delta$ & & 2.70 & 2.90 & 3.24 & & 2.63 & 3.58 & 4.80 & & 4.97 & & 2.97 & & 3.10 & 7.40 \\
\hline & $\mathrm{H}$ & D2 & & JHH & $A B$ & $13 A B$ & $12 \mathrm{AC}$ & & $A D$ & $13 \mathrm{CE}$ & $7 \mathrm{EF}$ & 12 & FG & 10 & A2B 2 & 12.5 & A2B2 & 12.5 & \\
\hline & & & & JHH & $A C$ & $7 \mathrm{BC}$ & $2 \mathrm{BC}$ & & $B D$ & $7 \mathrm{DE}$ & $13 \mathrm{FG}$ & 10 & & & & & & & \\
\hline & & & & JHH & $A D$ & $13 \mathrm{BD}$ & $7 \mathrm{CD}$ & 12 & $C D$ & $12 \mathrm{EF}$ & 12 & & & & & & & & \\
\hline & & & & JHH & & & CE & & DE & 13 & & & & & & & & & \\
\hline & & & & JHP & $\mathrm{AH}$ & $6 \mathrm{BH}$ & $22 \mathrm{CH}$ & 15 & $\mathrm{DH}$ & $0 \mathrm{EH}$ & $12 \mathrm{FH}$ & & $\mathrm{GH}$ & 4 & A2D2 & & A2D2 & 10 & \\
\hline PCy2, C4 & PPh2 & PR2 & JPP & & H & A & B & C & & D & E & $\mathbf{F}$ & & G & & A2 & & B2 & C2 \\
\hline \multirow[t]{6}{*}{ C4-1 } & 47.3 & 78.4 & 33 & $\delta$ & & 3.19 & 2.57 & 2.74 & & 3.14 & 4.70 & 4.38 & & 4.83 & & 3.12 & & 3.32 & 7.30 \\
\hline & $\mathrm{H}$ & D2 & & JHH & $A B$ & $12 \mathrm{AB}$ & $12 \mathrm{AC}$ & & $A D$ & $13 \mathrm{CE}$ & $7 \mathrm{EF}$ & 11.7 & FG & 11.7 & A2B2 & 12.5 & A2B2 & 12.5 & \\
\hline & & & & JHH & $A C$ & $7 \mathrm{BC}$ & $2 B C$ & 13 & $B D$ & $7 \mathrm{DE}$ & $12.1 \mathrm{FG}$ & 11.7 & & & & & & & \\
\hline & & & & JHH & $A D$ & $13 \mathrm{BD}$ & $7 \mathrm{CD}$ & 12 & $C D$ & $12 \mathrm{EF}$ & 11.7 & & & & & & & & \\
\hline & & & & JHH & & & CE & & DE & 12.1 & & & & & & & & & \\
\hline & & & & JHP & $\mathrm{AH}$ & $6 \mathrm{BH}$ & $22 \mathrm{CH}$ & 15 & $\mathrm{DH}$ & $12 \mathrm{EH}$ & $12 \mathrm{FH}$ & & $\mathrm{GH}$ & 4 & A2D2 & & A2D2 & 5 & \\
\hline
\end{tabular}




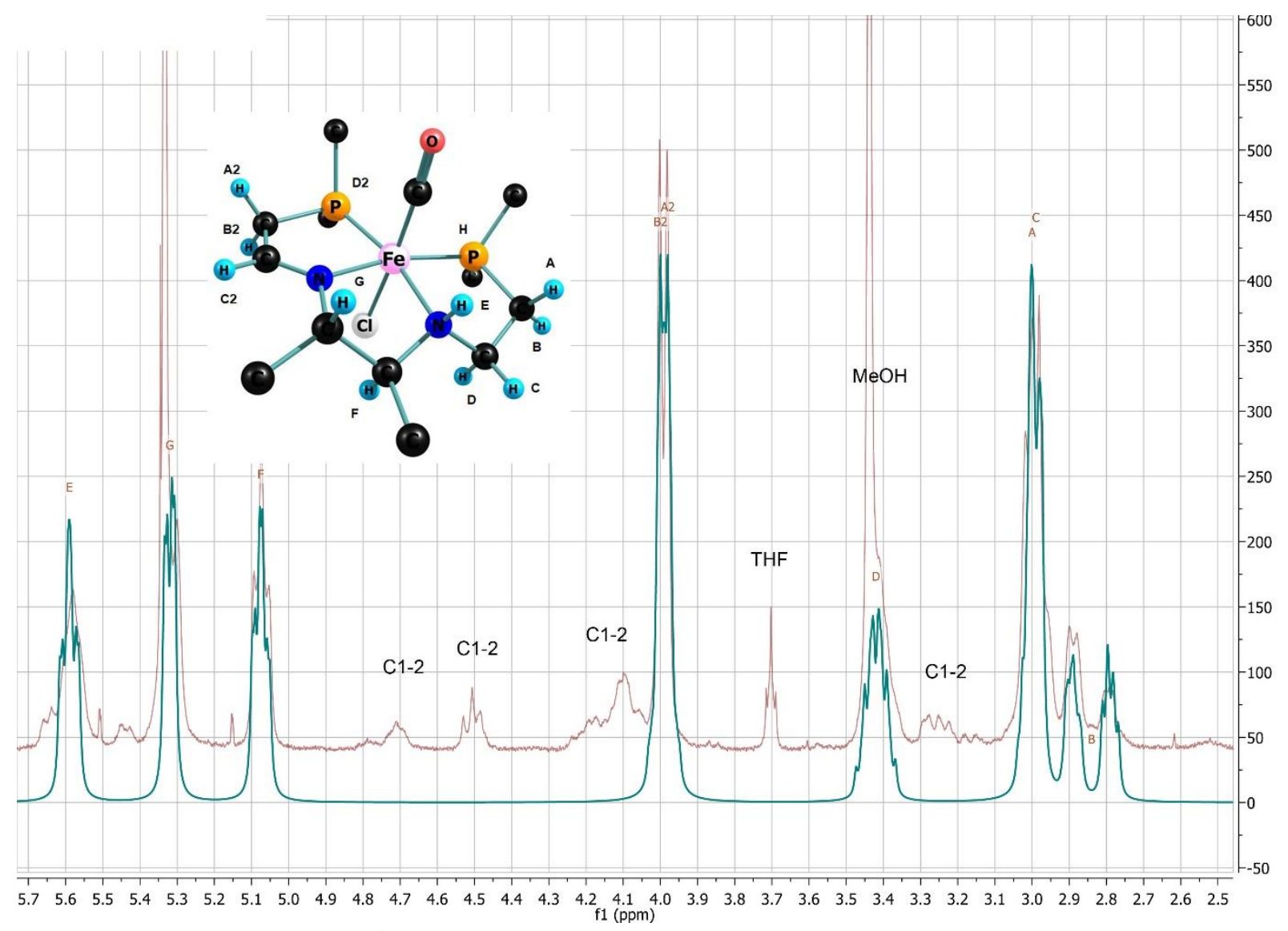

Figure S24. Simulation of the ${ }^{1} \mathrm{H}$ NMR spectrum at $500 \mathrm{MHz}$ of $\mathbf{C 1}\left(\mathrm{PR}_{2}=\mathrm{PPh}_{2}\right)$ in $\mathrm{CD}_{2} \mathrm{Cl}_{2}$. The parameters used are listed in Table S1. A small amount of a second isomer, C1-2, is present in this sample as indicated. 


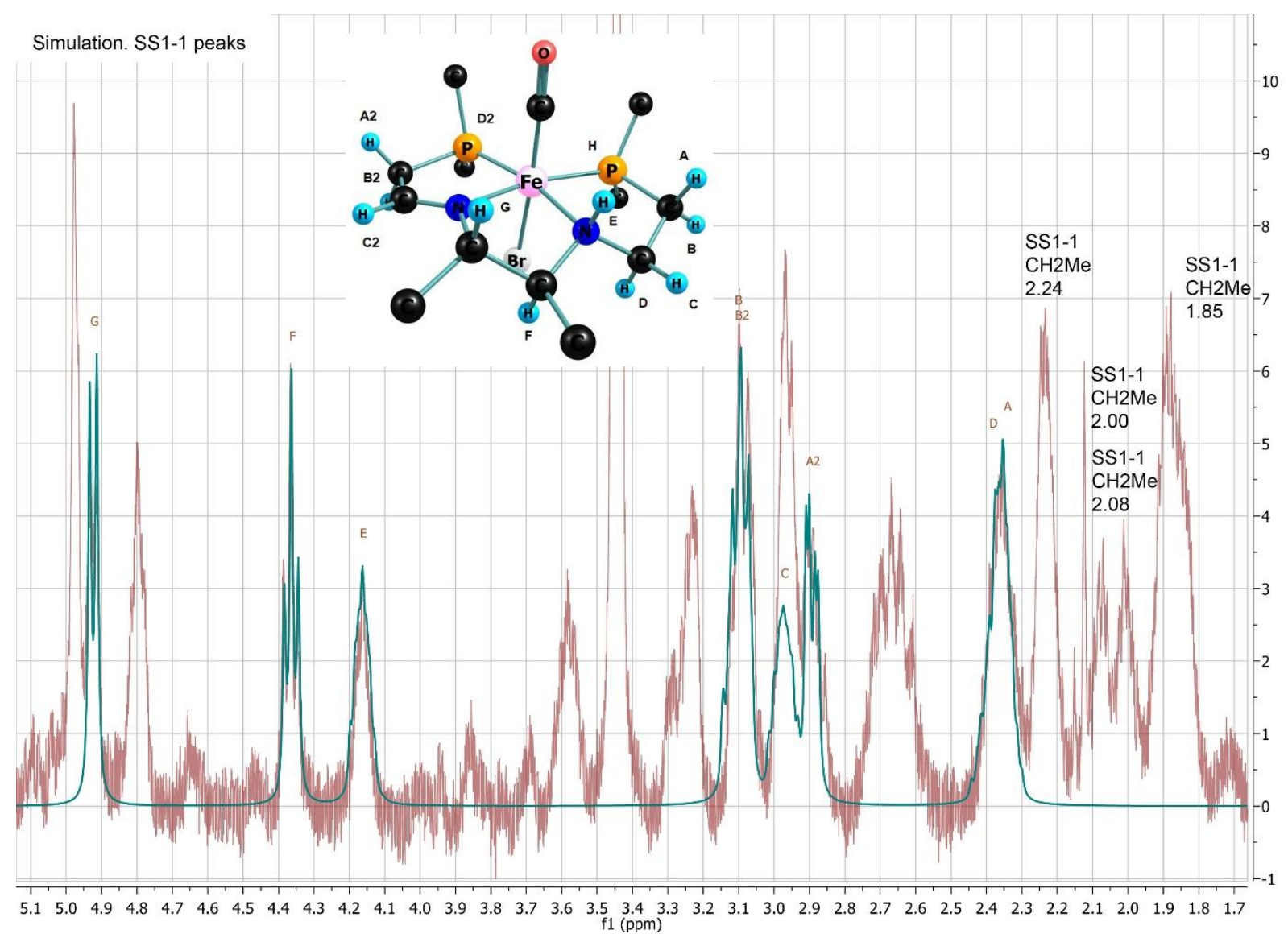

Figure S25. Simulation of the $600 \mathrm{MHz}{ }^{1} \mathrm{H}$ NMR spectrum of the peaks due to isomer $1,(S, S)-1-1$ (PR2 $\left.=\mathrm{PEt}_{2}\right)$ in $\mathrm{CD}_{2} \mathrm{Cl}_{2}$. The parameters used are listed in Table $\mathrm{S} 2$. The other peaks are from isomer $(\boldsymbol{S}, \boldsymbol{S})-$ 1-2 as in Figure $\mathbf{S 2 6}$. 


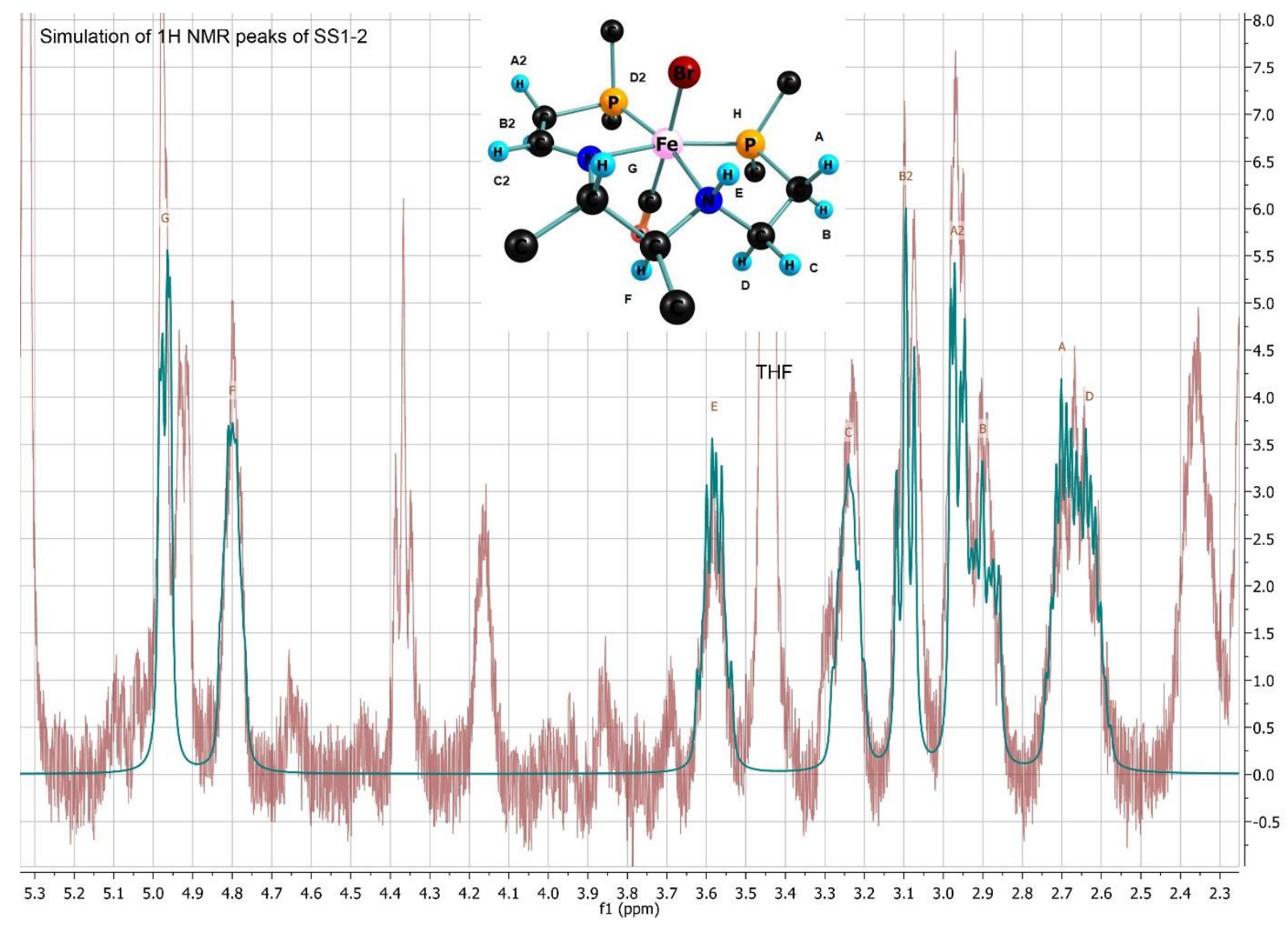

Figure S26. Simulation of the $600 \mathrm{MHz}^{1} \mathrm{H}$ NMR spectrum of the peaks due to isomer 2, (S,S)-1-2 $\left(\mathrm{PR}_{2}\right.$ $\left.=\mathrm{PEt}_{2}\right)$ in $\mathrm{CD}_{2} \mathrm{Cl}_{2}$. The parameters used are listed in Table $\mathrm{S} 1$. The other peaks are from isomer $(\mathbf{S}, \boldsymbol{S})$ 1-1 as in Figure $\mathbf{S 2 5 .}$ 


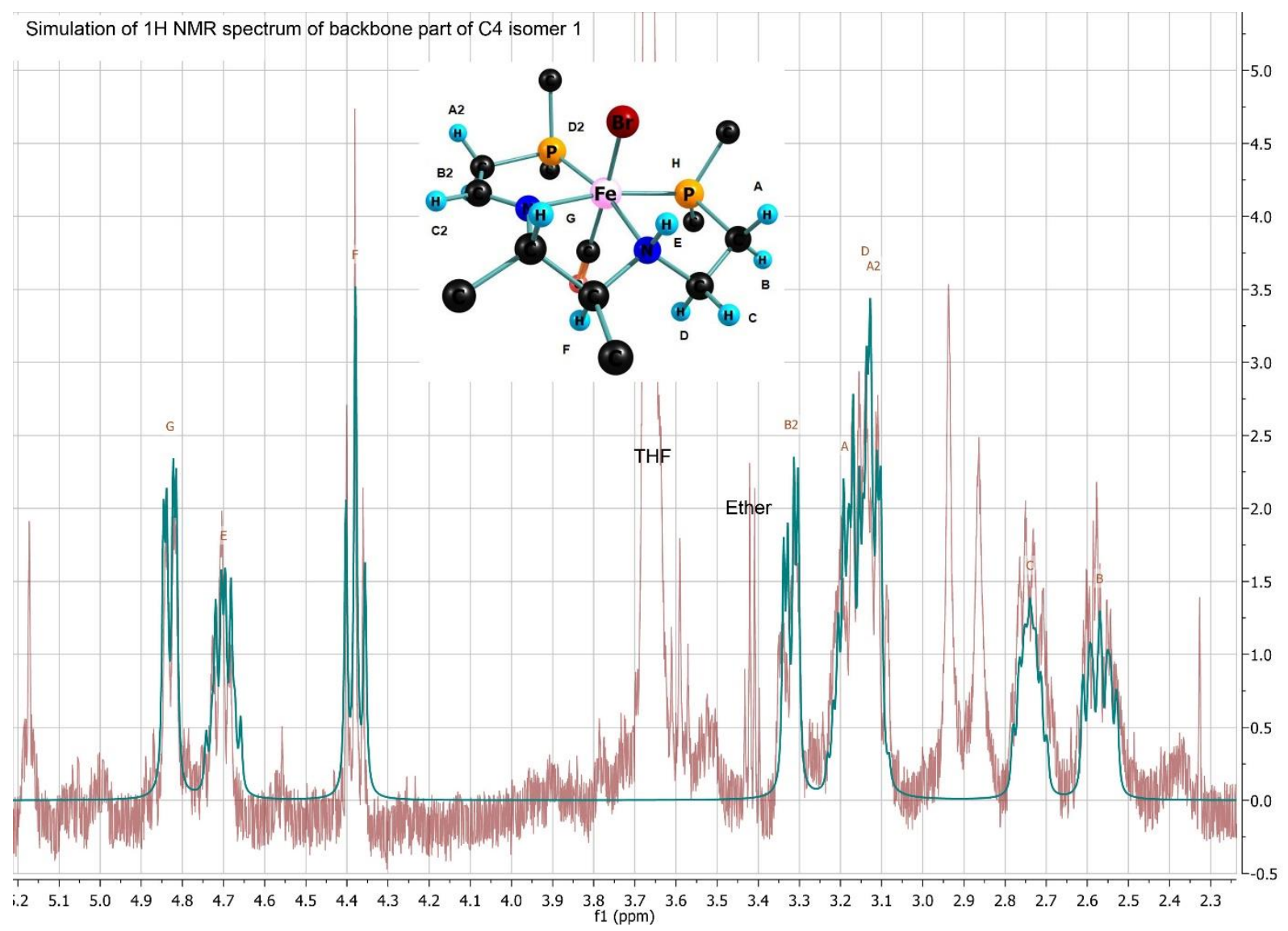

Figure S27. Simulation of the $600 \mathrm{MHz}{ }^{1} \mathrm{H}$ NMR spectrum of $\mathrm{C} 4\left(\mathrm{PR}_{2}=\mathrm{PCy}_{2}\right)$ in $\mathrm{CD}_{2} \mathrm{Cl}_{2}$. The parameters used are listed in Table S2. This sample had $75 \%$ of a trans isomer $\mathbf{C 4 - 1}$ and $25 \%$ of a second isomer, C4-1, possibly with a cis- $\beta$ structure since this is the isomer that crystallized from solution. The other peaks are from the second isomer and solvent impurities. This sample was used for the catalytic runs described here and in reference $3 c$ of the main article. 


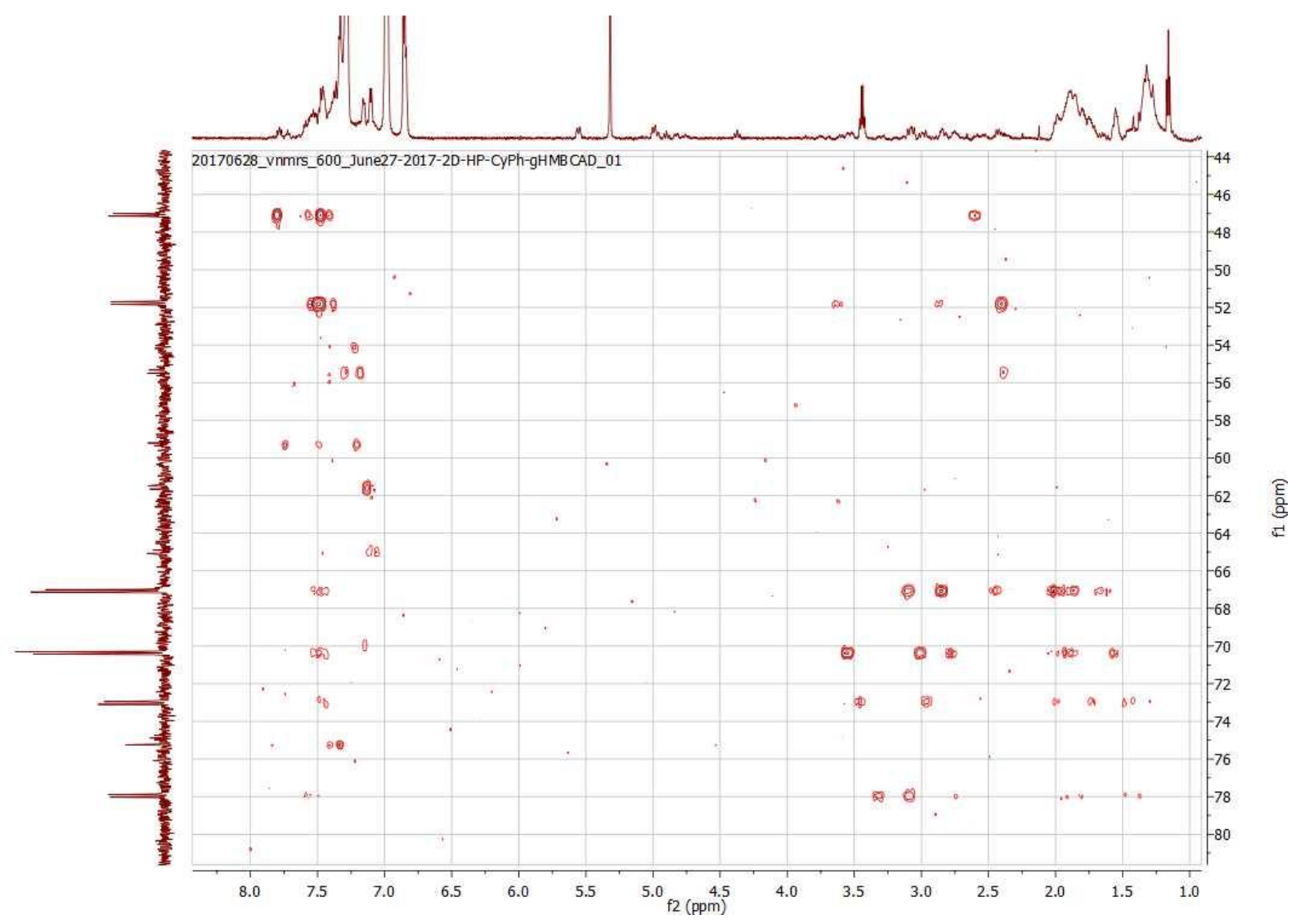

Figure S28. gHBMC ${ }^{1} \mathrm{H}^{3}{ }^{31} \mathrm{P}$ NMR spectrum $\left(600 \mathrm{MHz}, \mathrm{CD}_{2} \mathrm{Cl}_{2}\right)$ of a sample of $\mathrm{C4}$ mixed with some trans- $\left[\mathrm{FeBr}(\mathrm{CO})\left(\mathrm{PCy}_{2} \mathrm{CH}_{2} \mathrm{CH}=\mathrm{NCHPhCHPhN}=\mathrm{CHCH}_{2} \mathrm{PCy}_{2}\right)\right] \mathrm{BPh}_{4}(67,70 \mathrm{ppm}$, see reference $6 \mathrm{a}$ of the main article) which formed in the synthesis due to the presence of a dpen impurity. In this case both $E(47,78 \mathrm{ppm})$ and $E^{\prime}(72,52 \mathrm{ppm})$ isomers of $\mathbf{C} 4$ are observed under the conditions of the synthesis. 
Full table of catalytic results<smiles>CCC(=O)c1ccccc1C(=O)c1ccccc1</smiles>

A1

K1

K2

K3<smiles>CC(=O)c1ccc(Cl)cc1</smiles>

$\mathrm{K} 4$<smiles>CC(=O)c1ccccc1Cl</smiles>

K5<smiles>CC(=O)c1ccc(C(C)=O)cc1</smiles><smiles>CC(=O)/C=C/c1ccccc1</smiles>

Figure S29: Substrate Scope.

Table S2: Full catalytic results for the ATH of substrates in Figure S29.

\begin{tabular}{|c|c|c|c|c|c|c|}
\hline Entry & Subst. & Catalyst & $\begin{array}{l}\text { Conv. } \\
\text { (\%) }\end{array}$ & $\begin{array}{l}\text { Time } \\
\text { (min) }\end{array}$ & $\begin{array}{l}\text { e.e. } \\
\text { (\%) }\end{array}$ & TON \\
\hline 1 & $\mathrm{~A}_{1}$ & $(S, S)-1$ & $>99$ & 1 & N/A & 500 \\
\hline $2^{[b]}$ & & $C_{4}$ & $>99$ & 1 & $\mathrm{~N} / \mathrm{A}$ & 500 \\
\hline 3 & & $(S, S)-2$ & $>99$ & 1 & N/A & 500 \\
\hline 4 & & $\mathrm{C}_{1}$ & $>99$ & 1 & N/A & 500 \\
\hline 5 & $\mathrm{~K}_{1}$ & $(S, S)-1$ & 78 & 40 & 48 & 390 \\
\hline $6^{[b]}$ & & $C_{4}$ & 84 & 40 & 83 & 420 \\
\hline 7 & & $(S, S)-2$ & 78 & 40 & 80 & 390 \\
\hline $8^{[\mathrm{a}]}$ & & $(S, S)-1$ & 87 & 120 & 84 & 5325 \\
\hline $9^{[\mathrm{a}] \mathrm{b}]}$ & & $\mathrm{C}_{4}$ & 71 & 120 & 98 & 346 \\
\hline $10^{[a]}$ & & $(S, S)_{-2}$ & 67 & 120 & 89 & 4101 \\
\hline 11 & $\mathrm{~K}_{2}$ & $(S, S)-1$ & 79 & 3 & 86 & 395 \\
\hline $12^{[b]}$ & & $\mathrm{C}_{4}$ & 80 & 60 & 94 & 400 \\
\hline 13 & & $(S, S)_{-2}$ & 82 & 30 & 90 & 410 \\
\hline 14 & & $\mathrm{C}_{1}$ & 82 & 10 & 31 & 410 \\
\hline 15 & $\mathrm{~K}_{3}$ & $(S, S)_{-1}$ & 52 & 60 & 12 & 260 \\
\hline $16^{[\mathrm{b}]}$ & & $C_{4}$ & o & 60 & N/A & o \\
\hline 17 & & $(S, S)-2$ & o & 60 & N/A & o \\
\hline 18 & & $\mathrm{C}_{1}$ & 88 & 40 & 40 & 440 \\
\hline 19 & $\mathrm{~K}_{4}$ & $(S, S)_{-1}$ & 90 & 10 & 38 & 450 \\
\hline $20^{[b]}$ & & $\mathrm{C}_{4}$ & 92 & 10 & 52 & 460 \\
\hline 21 & & $(S, S)-2$ & 92 & 10 & 58 & 460 \\
\hline 22 & & $C_{1}$ & 90 & 3 & 6 & 450 \\
\hline 23 & $\mathrm{~K}_{5}$ & $(S, S)_{-1}$ & $>99$ & 1 & 90 & 500 \\
\hline $24^{[b]}$ & & $C_{4}$ & $>99$ & 10 & 94 & 500 \\
\hline 25 & & $(S, S)-2$ & $>99$ & 20 & 84 & 500 \\
\hline 26 & & $C_{1}$ & $>99$ & 1 & 94 & 500 \\
\hline 27 & K6 & $(S, S)-1$ & 63 & 60 & 86 & 15 \\
\hline $28^{[b]}$ & & $C_{4}$ & 38 & 40 & 95 & 190 \\
\hline 29 & & $(S, S)-2$ & $>99$ & 30 & 93 & oo \\
\hline
\end{tabular}




\begin{tabular}{|c|c|c|c|c|c|c|}
\hline 30 & & $C_{1}$ & $>99$ & 1 & 91 & 500 \\
\hline 31 & $\mathrm{~K}_{7}$ & $(S, S)-1$ & 62 & 20 & 15 & 310 \\
\hline $32^{[\mathrm{b}]}$ & & $C_{4}$ & 60 & 20 & 65 & 300 \\
\hline 33 & & $(S, S)-2$ & 60 & 40 & 63 & 300 \\
\hline 34 & & $\mathrm{C}_{1}$ & 60 & 30 & 3 & 300 \\
\hline 35 & K8 & $(S, S)-1$ & $>99$ & 3 & & 500 \\
\hline $36^{[\mathrm{b}]}$ & & $\mathrm{C}_{4}$ & $>99$ & 20 & & 500 \\
\hline 37 & & $(S, S)-2$ & $>99$ & 30 & & 500 \\
\hline 38 & & $\mathrm{C}_{1}$ & $>99$ & 2 & & 500 \\
\hline 39 & K9 & $(S, S)-1$ & 0 & 60 & N/A & 0 \\
\hline $40^{[\mathrm{b}]}$ & & $\mathrm{C}_{4}$ & 0 & 60 & N/A & 0 \\
\hline 41 & & $(S, S)-2$ & 0 & 60 & N/A & 0 \\
\hline 42 & & $\mathrm{Cl}_{1}$ & 0 & 60 & N/A & 0 \\
\hline 43 & Kio & $(S, S)-1$ & 63 & 60 & 27 & 315 \\
\hline $44^{[\mathrm{b}]}$ & & $\mathrm{C}_{4}$ & 44 & 60 & 43 & 220 \\
\hline 45 & & $(S, S)-2$ & 75 & 40 & 55 & 375 \\
\hline 46 & & $C_{1}$ & 90 & 20 & 23 & 450 \\
\hline 47 & K11 & $(S, S)-1$ & 33 & 60 & N/A & 165 \\
\hline $4^{[\mathrm{b}]}$ & & $\mathrm{C}_{4}$ & o & 60 & $\mathrm{~N} / \mathrm{A}$ & o \\
\hline 49 & & $(S, S)-2$ & $>99$ & 2 & $\mathrm{~N} / \mathrm{A}$ & 500 \\
\hline 50 & & $C_{1}$ & $>99$ & 50 & N/A & 500 \\
\hline 51 & K12 & $(S, S)-1$ & 81 & 30 & 23 & 405 \\
\hline $5^{2^{[b]}}$ & & $\mathrm{C}_{4}$ & 88 & 40 & 82 & 440 \\
\hline 53 & & $(S, S)-2$ & 84 & 40 & 59 & 420 \\
\hline 54 & & $C_{1}$ & 76 & 20 & 3 & 380 \\
\hline 55 & $\mathrm{~K}_{13}$ & $(S, S)-1$ & o & 60 & N/A & o \\
\hline $5^{6^{[b]}}$ & & $\mathrm{C}_{4}$ & 11 & 60 & 53 & 55 \\
\hline 57 & & $(S, S)-2$ & o & $6 o$ & $\mathrm{~N} / \mathrm{A}$ & o \\
\hline 58 & & $\mathrm{Cl}_{1}$ & o & 60 & N/A & o \\
\hline 59 & $\mathrm{~K}_{14}$ & $(S, S)-1$ & 90 & 20 & $\mathrm{~N} / \mathrm{A}$ & 450 \\
\hline $60^{[b]}$ & & $C_{4}$ & 89 & 20 & N/A & 445 \\
\hline 61 & & $(S, S)-2$ & 91 & 20 & N/A & 455 \\
\hline 62 & & $C_{1}$ & 89 & 1 & N/A & 445 \\
\hline 63 & $\mathrm{~K}_{15}$ & $(S, S)_{-1}$ & 57 & 5 & 4 & 285 \\
\hline $64^{[\mathrm{b}]}$ & & $\mathrm{C}_{4}$ & 40 & 20 & 5 & 200 \\
\hline 65 & & $(S, S)-2$ & 22 & 30 & 46 & 110 \\
\hline 66 & & $C_{1}$ & 78 & 50 & 3 & 390 \\
\hline
\end{tabular}

${ }^{[a]} \mathrm{C} / \mathrm{B} / \mathrm{S}=1 / 2 / 6121 ;{ }^{[b]}$ from reference 12

\section{GC Traces for reduction of acetophenone K1}

\section{$(S, S)-1$}

GC analysis conditions: Oven temperature $130^{\circ} \mathrm{C}$

Retention time: $(R)$-isomer $=6.693 ;(S)$-isomer $=7.193 ;$ starting material $=4.093$. 


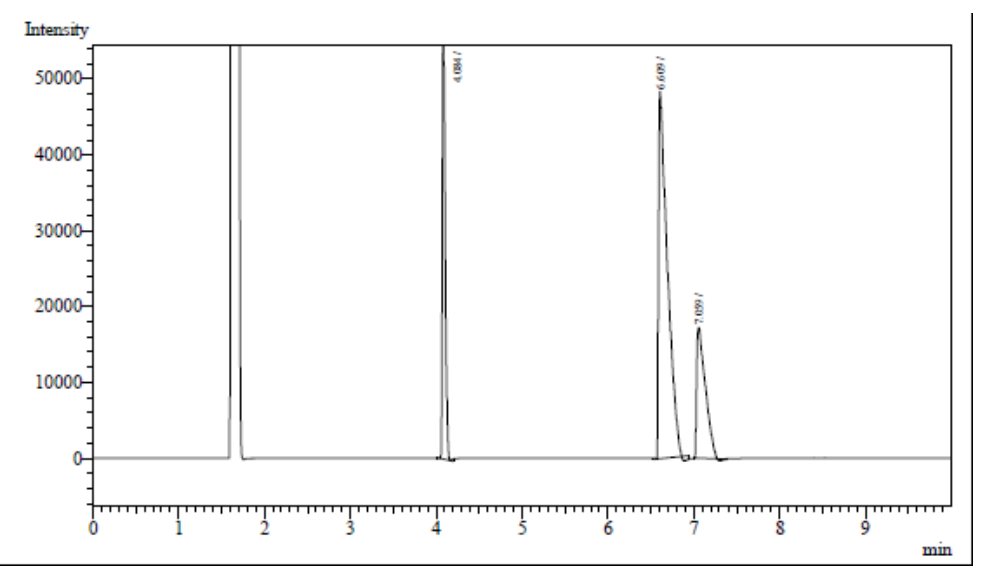

$(S, S)-2$

GC analysis conditions: Oven temperature $130{ }^{\circ} \mathrm{C}$

Retention time: $(R)$-isomer $=6.693 ;(S)$-isomer $=7.193 ;$ starting material $=4.093$.

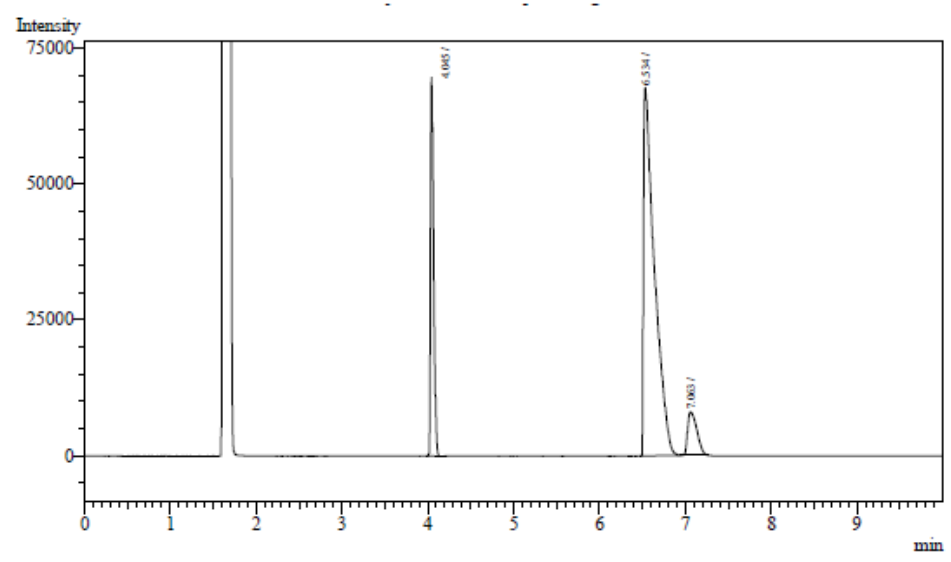

$(S, S)-1$

GC analysis conditions: Oven temperature $130^{\circ} \mathrm{C}$

Retention time: $(R)$-isomer $=6.693 ;(S)$-isomer $=7.193 ;$ starting material $=4.093$.

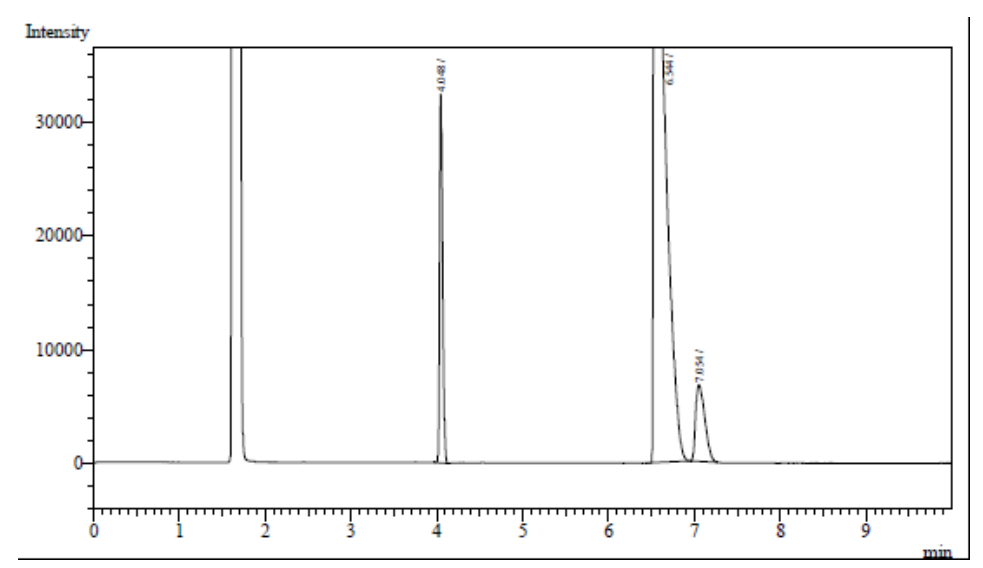

$(S, S)-2$

GC analysis conditions: Oven temperature $130^{\circ} \mathrm{C}$

Retention time: $(R)$-isomer $=6.693 ;(S)$-isomer $=7.193 ;$ starting material $=4.093$. 


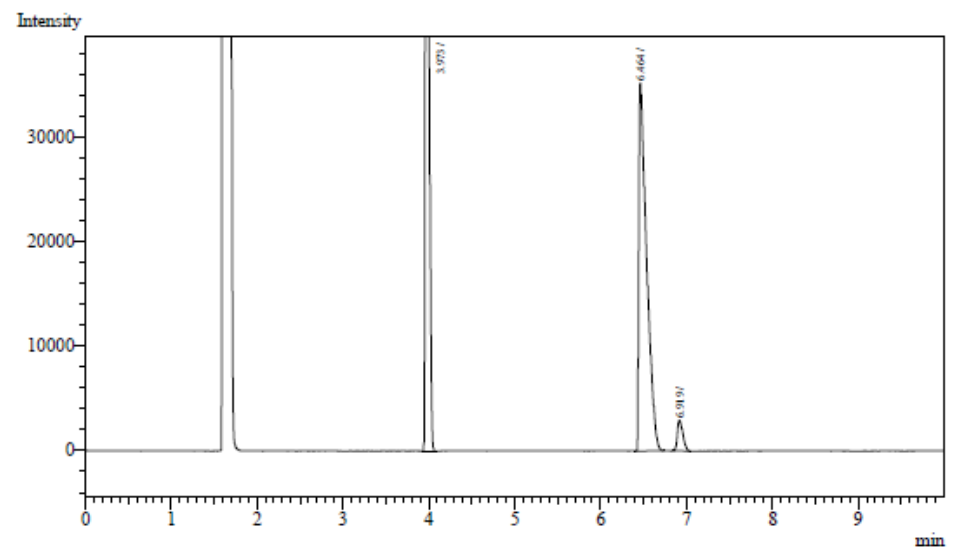

GC Traces for Table 3 of the Main Article.

Propiophenone $\mathbf{K 2}$

$\mathrm{GC}$ analysis conditions: Oven temperature $120^{\circ} \mathrm{C}$

Retention time: $(R)$-isomer $=10.966 ;(S)$-isomer $=11.992 ;$ starting material $=5.349$.

Entry $1(S, S)-4$

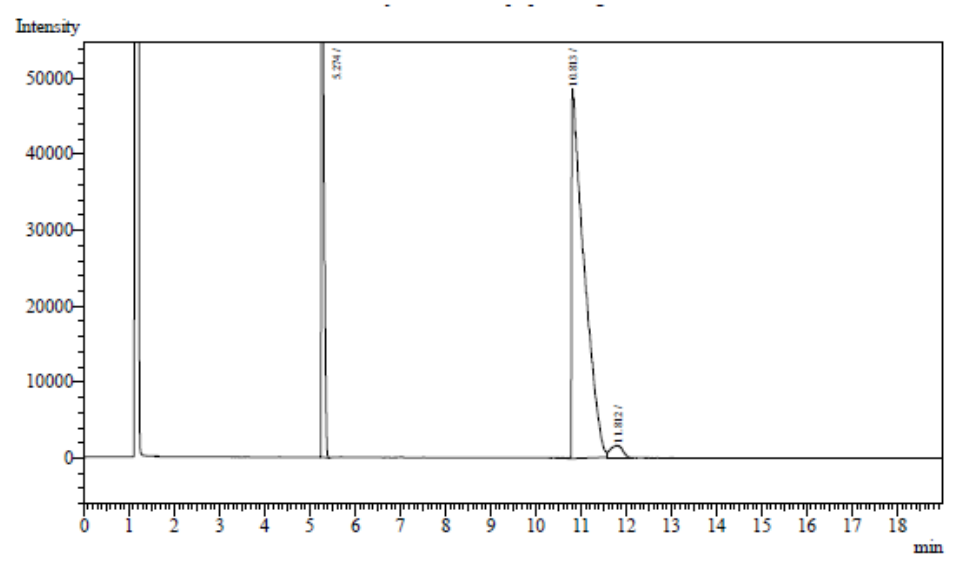

Entry $3(S, S)-2$

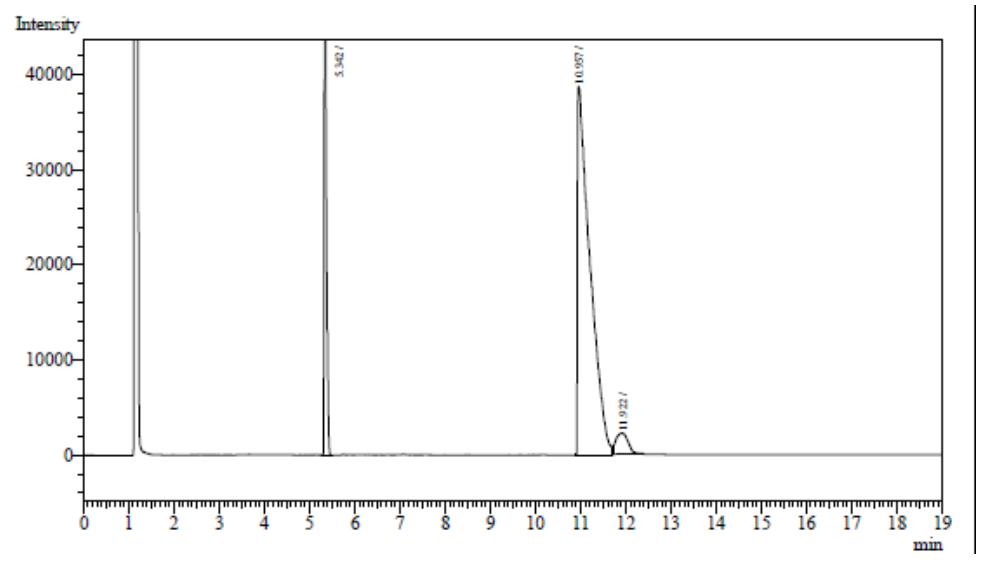

Butyrophenone K3

GC analysis conditions: Oven temperature $140{ }^{\circ} \mathrm{C}$ 
Retention time: $(R)$-isomer $=47.884 ;(S)$-isomer $=50.454 ;$ starting material $=11.762$.

Entry $4(S, S)-1$

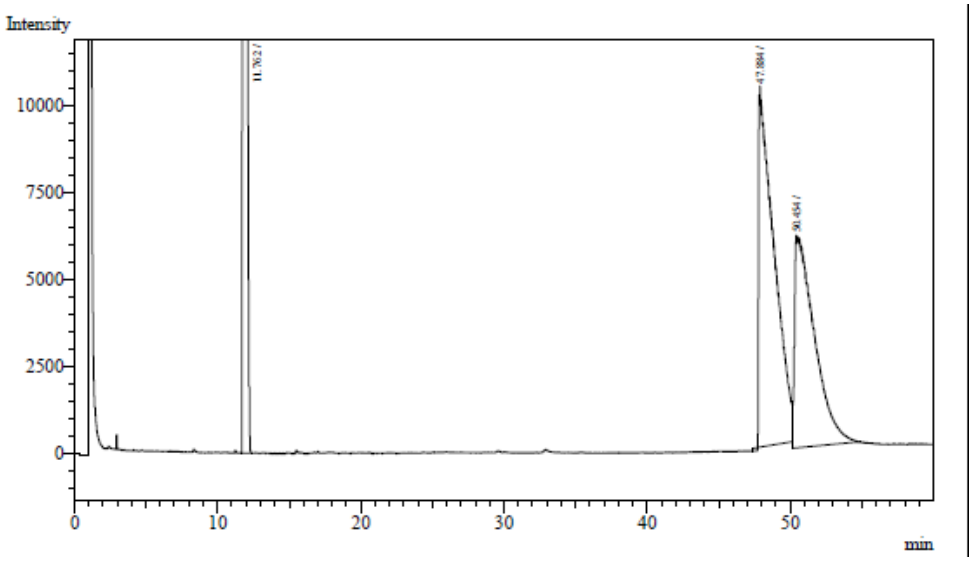

p-Chloroacetophenone K4

GC analysis conditions: Oven temperature $145^{\circ} \mathrm{C}$

Retention time: $(R)$-isomer $=4.737 ;(S)$-isomer $=5.213 ;$ starting material $=2.588$

\section{Entry $7(S, S)-2$}

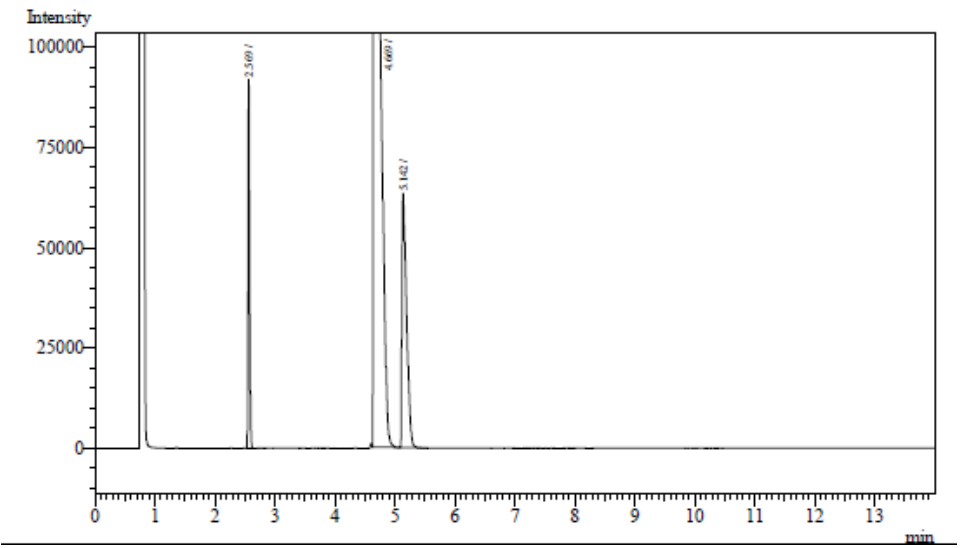

o-Chloroacetophenone K5

GC analysis conditions: Oven temperature $145^{\circ} \mathrm{C}$

Retention time: $(R)$-isomer $=5.654 ;(S)$-isomer $=6.924 ;$ starting material $=2.752$.

Entry $8(S, S)-1$ 


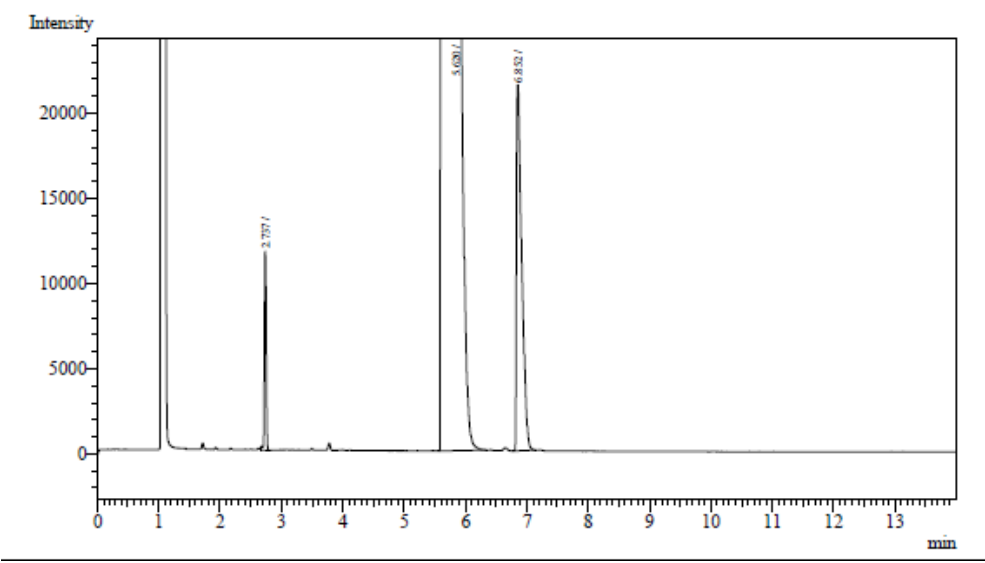

\section{$3^{\prime}, 5^{\prime}-\left(\mathrm{CF}_{3}\right)_{2}$ acetophenone $\mathrm{K} 6$}

GC analysis conditions: Oven temperature $140^{\circ} \mathrm{C}$

Retention time: $(R)$-isomer $=2.735 ;(S)$-isomer $=2.606$; starting material $=1.399$.

\section{Entry $11(S, S)-2$}

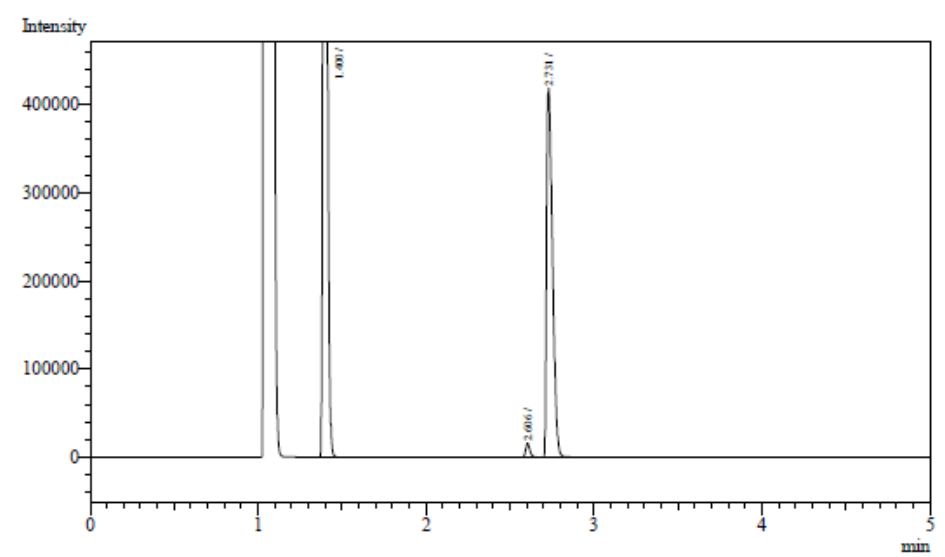

\section{p-Methylacetophenone K7}

GC analysis conditions: Oven temperature $130{ }^{\circ} \mathrm{C}$

Retention time: $(R)$-isomer $=6.034 ;(S)$-isomer $=6.637 ;$ starting material $=4.142$.

\section{Entry $15(S, S)-2$}

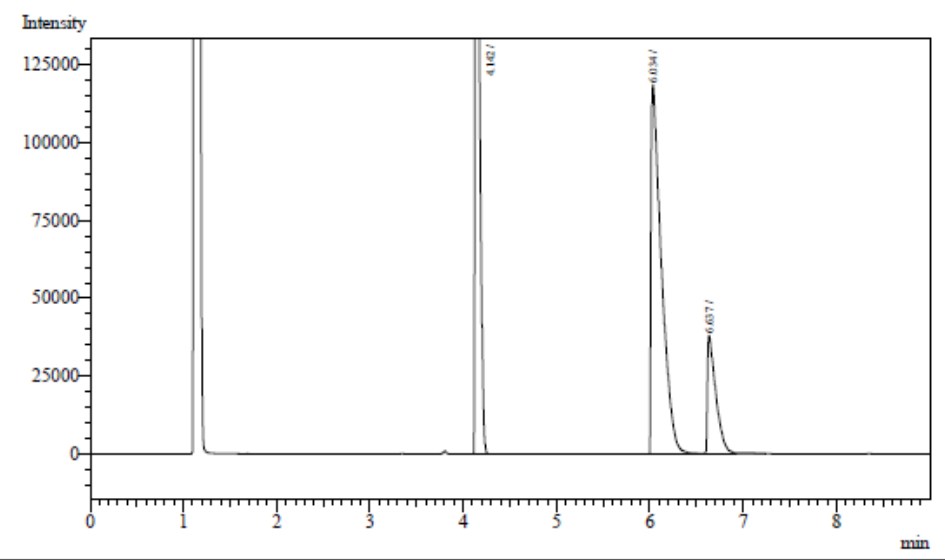

2-Acetylpyridine $\mathrm{K} 8$ 
GC analysis conditions: Oven temperature $100^{\circ} \mathrm{C}$

Retention time: $(R)$-isomer $=13.779 ;(S)$-isomer $=14.208 ;$ starting material $=5.865$.

\section{Entry $16(S, S)-1$}

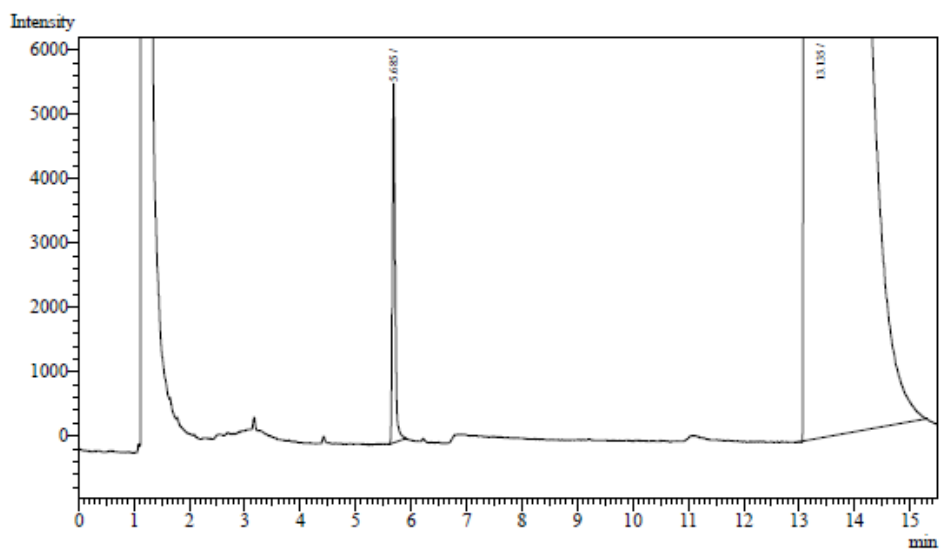

\section{Entry $17(S, S)-2$}

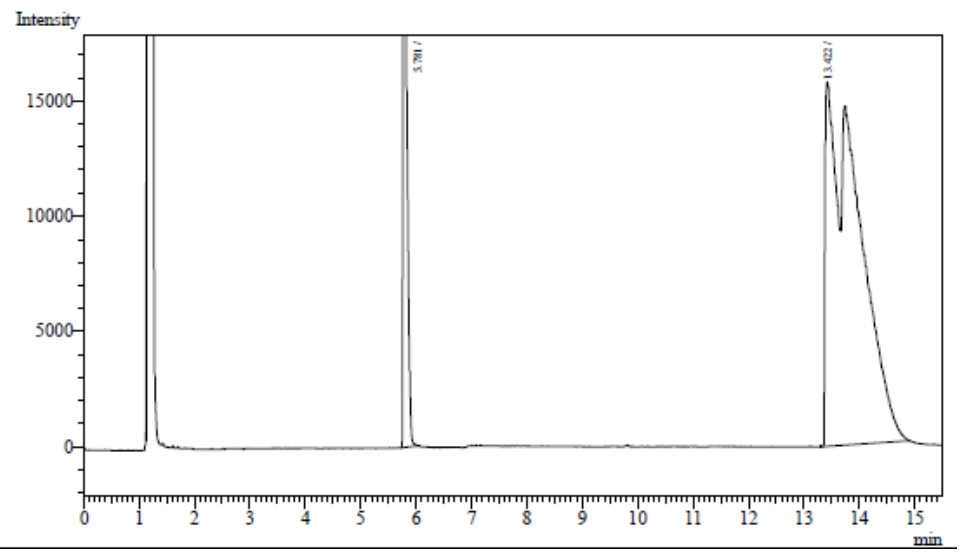

\section{2-acetylfuran K9}

GC analysis conditions: Oven temperature $90{ }^{\circ} \mathrm{C}$

Retention time: $(R)$-isomer $=8.827 ;(S)$-isomer $=9.263 ;$ starting material $=4.165$.

\section{3-Methylbutan-2-one K10}

GC analysis conditions: Oven temperature $60^{\circ} \mathrm{C}$

Retention time: $(R)$-isomer $=9.41 ;(S)$-isomer $=9.92 ;$ starting material $=3.74$.

Entry $21(S, S)-2$ 


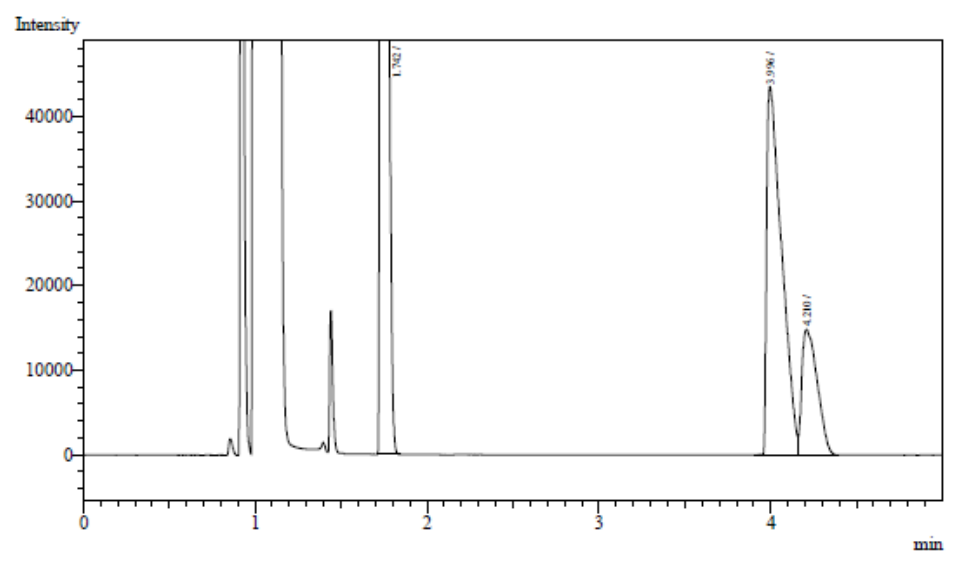

\section{Cyclohexanone K11}

GC analysis conditions: Oven temperature $110^{\circ} \mathrm{C}$

Retention time: alcohol product $=2.941 ;$ starting material $=2.285$.

\section{Entry $23(S, S)-1$}

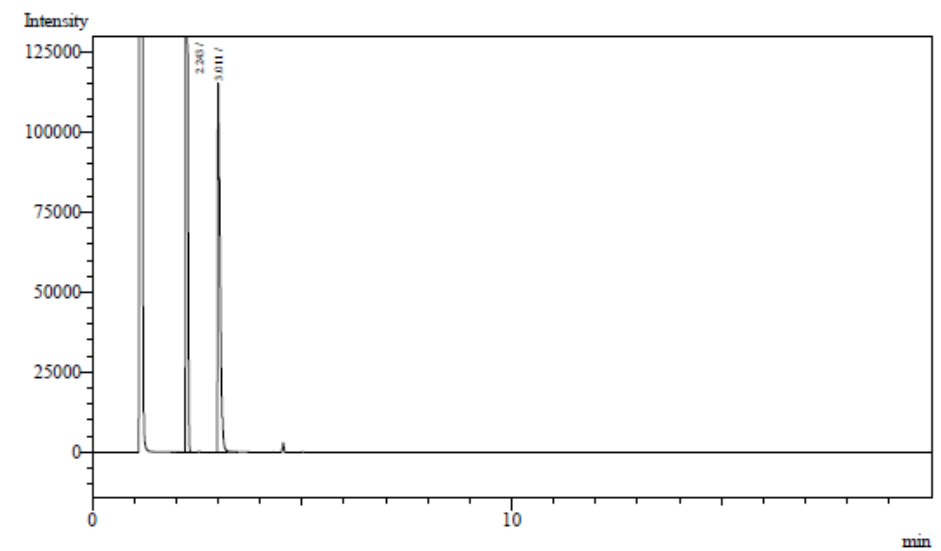

Entry $24(S, S)-2$

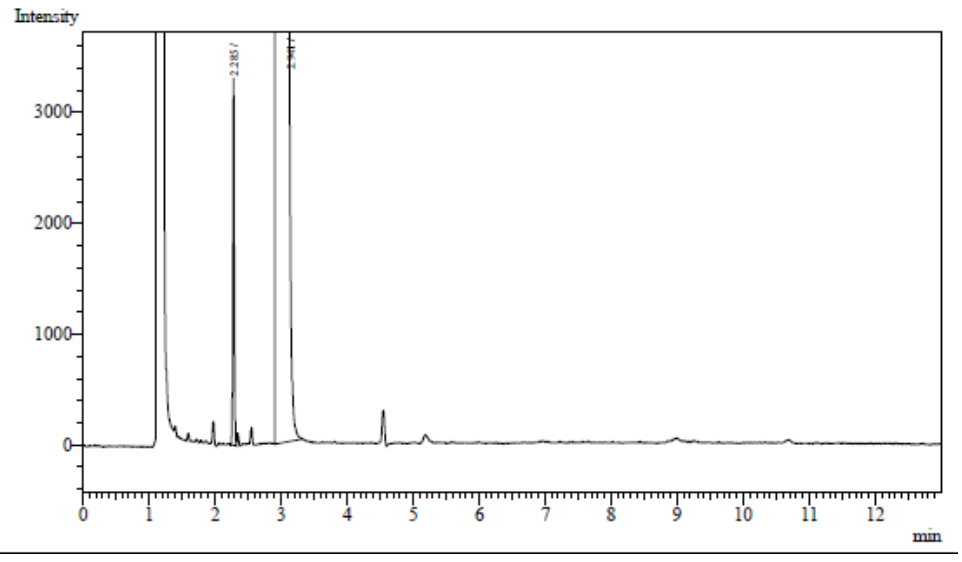

\section{2-Acetonaphthone K12}

GC analysis conditions: Oven temperature $150^{\circ} \mathrm{C}$

Retention time: $(R)$-isomer $=6.119 ;(S)$-isomer $=6.378$; starting material $=4.403$.

Entry $26(S, S)-1$ 


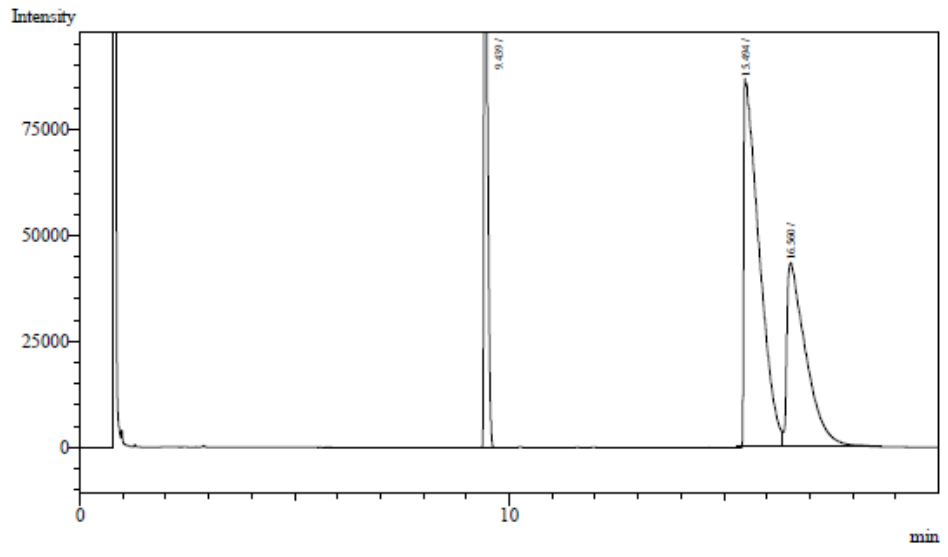

Entry $29(S, S)-2$

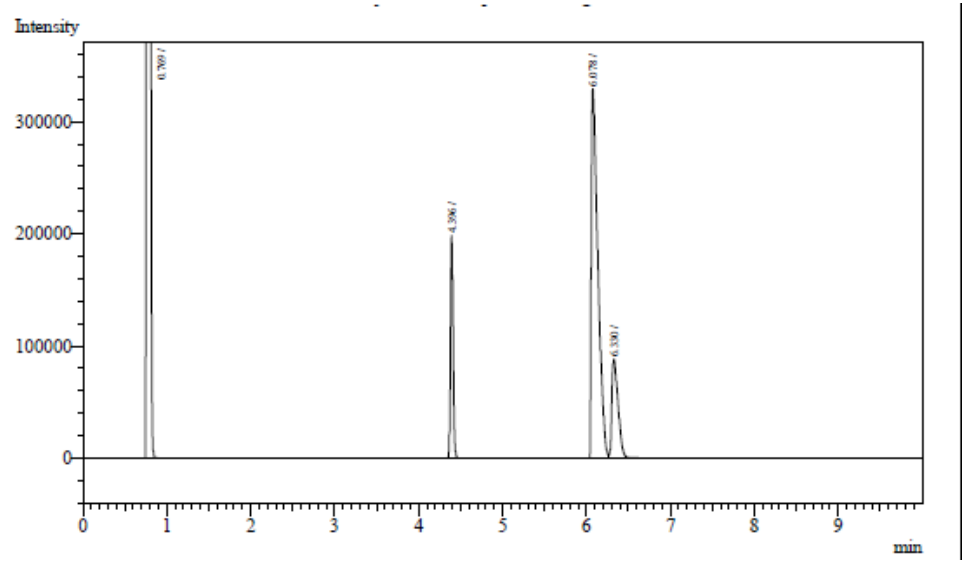

\section{Benzophenone K14}

GC analysis conditions: Oven temperature $180^{\circ} \mathrm{C}$

Retention time: product $=5.013 ;$ starting material $=3.228$.

\section{Entry $31(S, S)-1$}

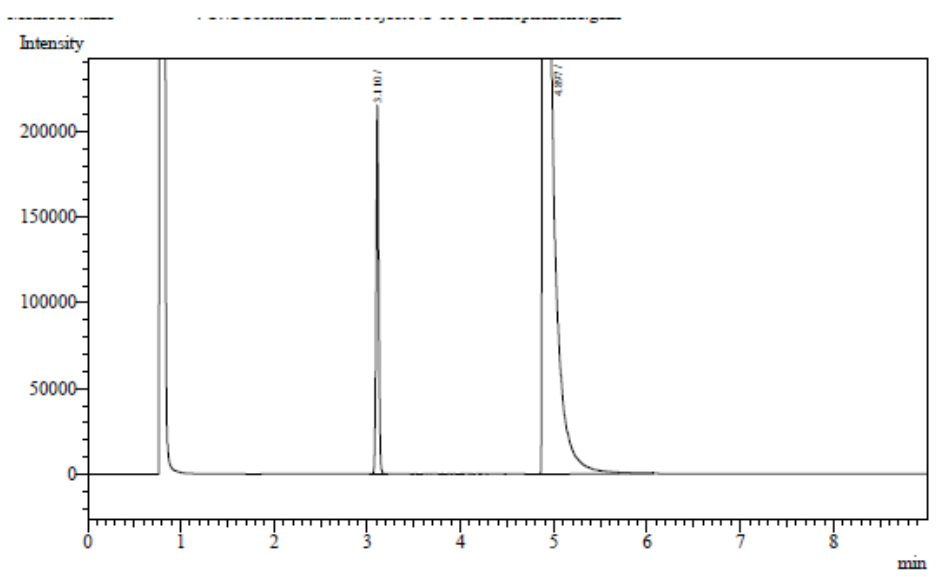




\section{Entry $33(S, S)-2$}

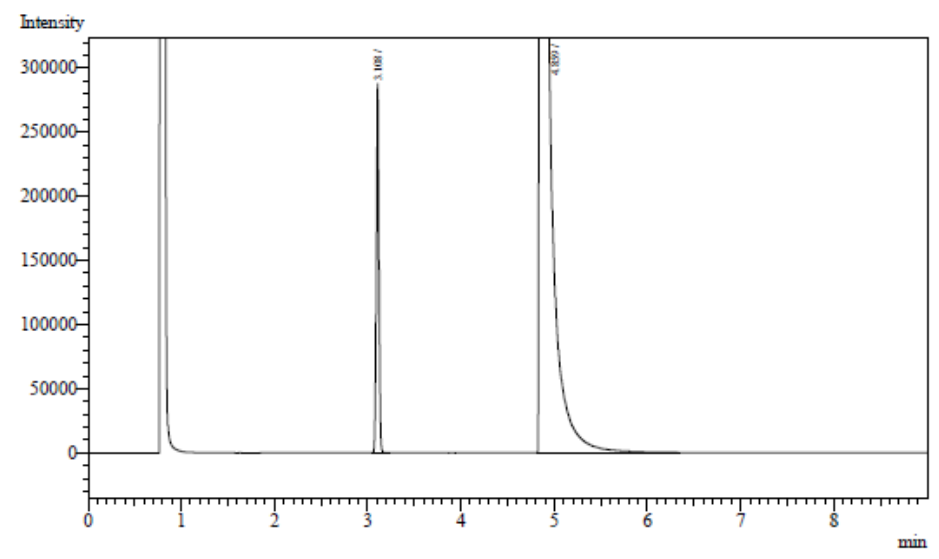

\section{Benzylidene acetone K15}<smiles>CCC(=O)/C=C/c1ccccc1</smiles>

(S,S)-2, $\mathrm{KOtBu}, \mathrm{iPrOH}$ $28^{\circ} \mathrm{C}$<smiles>C[C@H](O)CCc1ccccc1</smiles><smiles>CC(=O)CCc1ccccc1</smiles><smiles>CC(O)C=Cc1ccccc1</smiles>

GC analysis conditions: Oven temperature $125^{\circ} \mathrm{C}$

Retention time: $(R)$-isomer $=15.743 ;(S)$-isomer $=16.157 ;$ RED- $(R)$-isomer $=10.677 ;$ RED- $(S)$-isomer $=$ 11.051; REDK $=6.416$; starting material $=12.175$.

\section{Entry $35(S, S)-2$}

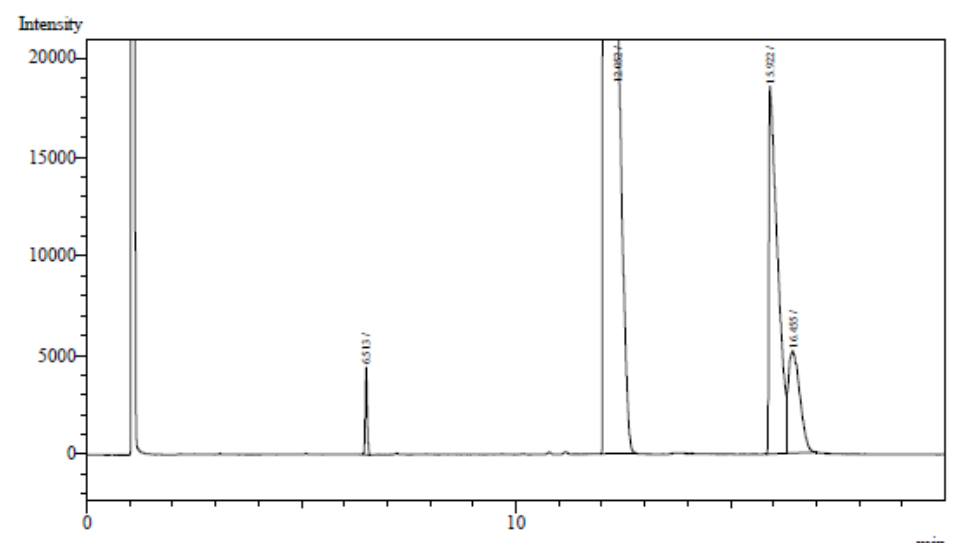




\section{DFT Calculations.}

DFT calculations were performed using Gaussian 09 (Rev. D.01) ${ }^{1}$ at the $\mathrm{M06L}^{2} / \mathrm{TZVP}^{3} / \mathrm{TZVPfit}$ level of theory. Either normal (opt) or tight (opt = tight) convergence criteria were used for all optimizations, and a pruned $(99,590)$ integration grid was used throughout (grid = ultrafine). Optimizations were performed in a 2-propanol solvent continuum using the integral equation formalism polarizable continuum model (IEF-PCM) ${ }^{4,5}$ with radii and non-electrostatic terms from the SMD solvation model (scrf $=\mathrm{smd}$ ). ${ }^{6}$ Full vibrational and thermochemical analyses ( $1 \mathrm{~atm}, 298 \mathrm{~K}$ ) were performed on optimized structures to obtain solvent-corrected free energies $\left(G^{\circ}\right)$ and enthalpies $\left(H^{\circ}\right)$. Optimized ground states were found to have zero imaginary frequencies. Calculations were performed in part by the facilities of the Shared Hierarchical Academic Research Computing Network (SHARCNET) and Compute/Calcul Canada. ${ }^{7}$ Complexes trans-(S,S)-C1 and G1-1 have been reported elsewhere using the same level of theory. ${ }^{8}$

Scheme S1 and Tables S3 and S4 compare the calculated and observed energies and structures of the two isomers of the starting complex $\mathbf{C} 4$ that were observed in the crystalline state. ${ }^{9}$

Scheme S2 compares the energies of some of the possible isomers of the alkoxide complexes (the complete set of isomers is found in Scheme 4 of the main article) derived from the reaction of C4 with KOtBu and then 2propanol. The intermediate alkoxide complexes are found to be less stable than the corresponding hydrides produced from them.

Scheme S3 compares the energies of possible isomers of the amido and hydride complexes derived from the reaction of $\mathbf{C} \mathbf{1}$ with KOtBu and then isopropanol in an analogous fashion to those of $\mathbf{C} \mathbf{4}$ found in Scheme 4 of the main article. Some of these were not considered in the original DFT study of such complexes. ${ }^{8}$ Here too the calculations show that cis- $\beta$ hydride isomers cannot be ruled out as intermediates or resting states in catalysis.

1. Gaussian 09, Revision or B.01 or D.01, M. J. Frisch, G. W. Trucks, H. B. Schlegel, G. E. Scuseria, M. A. Robb, J. R. Cheeseman, G. Scalmani, V. Barone, B. Mennucci, G. A. Petersson, H. Nakatsuji, M. Caricato, X. Li, H. P. Hratchian, A. F. Izmaylov, J. Bloino, G. Zheng, J. L. Sonnenberg, M. Hada, M. Ehara, K. Toyota, R. Fukuda, J. Hasegawa, M. Ishida, T. Nakajima, Y. Honda, O. Kitao, H. Nakai, T. Vreven, J. A. Montgomery, Jr., J. E. Peralta, F. Ogliaro, M. Bearpark, J. J. Heyd, E. Brothers, K. N. Kudin, V. N. Staroverov, R.

Kobayashi, J. Normand, K. Raghavachari, A. Rendell, J. C. Burant, S. S. Iyengar, J. Tomasi, M. Cossi, N. Rega, J. M. Millam, M. Klene, J. E. Knox, J. B. Cross, V. Bakken, C. Adamo, J. Jaramillo, R. Gomperts, R. E. Stratmann, O. Yazyev, A. J. Austin, R. Cammi, C. Pomelli, J. W. Ochterski, R. L. Martin, K. Morokuma, V. G. Zakrzewski, G. A. Voth, P. Salvador, J. J. Dannenberg, S. Dapprich, A. D. Daniels, Ö. Farkas, J. B. Foresman, J. V. Ortiz, J. Cioslo

2. Y. Zhao, D. G. Truhlar, J. Chem. Phys., 2006, 125, 194101.

3. A. Schäfer, C. Huber, R. Ahlrichs, J. Chem. Phys., 1994, 100, 5829-5835.

4. J. Tomasi, B. Mennucci, E. Cancès, J. Molec. Struct.: THEOCHEM, 1999, 464, 211-226.

5. J. Tomasi, B. Mennucci, R. Cammi, Chem. Rev., 2005, 105, 2999-3094.

6. A. V. Marenich, C. J. Cramer, D. G. Truhlar, J. Phys. Chem. B, 2009, 113, 6378-6396.

7. www.sharcnet.ca.

8. Zuo, W.; Prokopchuk, D. E.; Lough, A. J.; Morris, R. H. ACS Catalysis 2016, 6, 301-314.

9. Smith, S. A. M.; Lough, A. J.; Morris, R. H. IUCrData 2017, https://doi.org/10.1107/S2414314617004527. 
Scheme S1. Ground state energies of the cis- $\beta$ isomers of C4 (relative to $\Delta-(S, S)-\mathrm{C4})$.

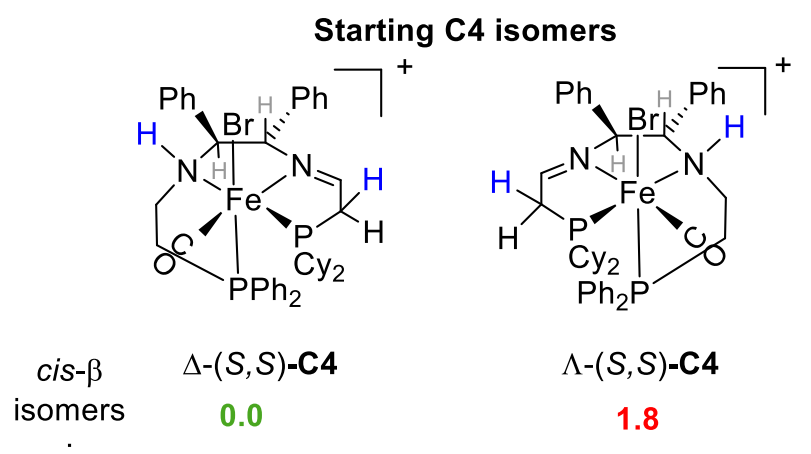

Table S3. Selected experimental and calculated metrical parameters for complex $\Delta$-cis- $\beta$-C4 $\mathbf{R}_{\mathbf{N H}}$.

\begin{tabular}{|c|c|c|}
\hline & Experimental & Calculated \\
\hline Parameter & \multicolumn{2}{|c|}{ Length $(\AA ̊)$} \\
\hline Fe1B-Br1B & $2.5183(8)$ & 2.564 \\
\hline Fe1B-P1B & $2.246(1)$ & 2.307 \\
\hline Fe1B-P2B & $2.260(2)$ & 2.278 \\
\hline Fe1B-N1B & $2.071(5)$ & 2.075 \\
\hline Fe1B-N2B & $1.989(4)$ & 2.010 \\
\hline Fe1B-C7B & $1.756(7)$ & 1.764 \\
\hline \multirow[t]{2}{*}{$\mathrm{N} 2 \mathrm{~B}-\mathrm{C} 5 \mathrm{~B}$} & $1.265(7)$ & 1.275 \\
\hline & \multicolumn{2}{|c|}{ Angle $\left({ }^{\circ}\right)$} \\
\hline P1B-Fe1B-P2B & $102.70(6)$ & 101.5 \\
\hline N1B-Fe1B-N2B & $82.4(2)$ & 81.7 \\
\hline N1B-Fe1B-P1B & $80.2(1)$ & 80.5 \\
\hline N1B-Fe1B-P2B & $165.7(1)$ & 165.6 \\
\hline N2B-Fe1B-P1B & 101.3(1) & 96.5 \\
\hline N2B-Fe1B-P2B & $83.3(1)$ & 83.9 \\
\hline C7B-Fe1B-Br1B & $85.1(2)$ & 86.4 \\
\hline
\end{tabular}

Table S4. Selected experimental and calculated metrical parameters for complex $\Lambda$-cis- $\beta-\mathbf{C} 4 \mathbf{S}_{\mathbf{N H}}$.

\begin{tabular}{lcc}
\hline Parameter & Experimental & Calculated \\
& \multicolumn{2}{c}{ Length $(\AA)$} \\
Fe1A-Br1A & $2.4970(8)$ & 2.565 \\
Fe1A-P1A & $2.240(1)$ & 2.278
\end{tabular}




$\begin{array}{ccc}\text { Fe1A-P2A } & 2.256(2) & 2.277 \\ \text { Fe1A-N1A } & 2.089(5) & 2.015 \\ \text { Fe1A-N2A } & 1.976(4) & 2.021 \\ \text { Fe1A-C7A } & 1.758(7) & 1.762 \\ \text { N2A-C5A } & 1.280(8) & 1.275 \\ & & \text { Angle }\left(^{\circ}\right) \\ \text { P1A-Fe1A-P2A } & 104.36(6) & 100.9 \\ \text { N1A-Fe1A-N2A } & 82.1(2) & 80.8 \\ \text { N1A-Fe1A-P1A } & 82.9(1) & 82.9 \\ \text { N1A-Fe1A-P2A } & 165.6(1) & 164.9 \\ \text { N2A-Fe1A-P1A } & 95.7(1) & 94.5 \\ \text { N2A-Fe1A-P2A } & 84.8(1) & 84.3 \\ \text { C7A-Fe1A-Br1A } & 87.5(2) & 85.8\end{array}$


Scheme $\mathbf{S 2}$ - Reactions of some of the possible eneamido structures (S,S)-4 (for a more complete set see Scheme $\mathbf{S} 4$ of the main article) with alcohol to give alkoxide isomers $(\mathbf{S}, \mathbf{S})-\mathbf{5}$ which lead to some of the possible hydride isomers $(\mathbf{S}, \mathbf{S})-\mathbf{3}$. Ground state energies in $\mathrm{kcal} / \mathrm{mol}$ (relative to $\Delta$-cis- $\beta-(S, S)$ 4A).

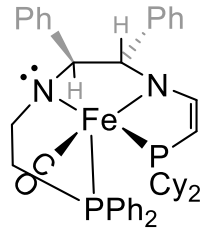

$(S, S)-4:$ $\Delta-\operatorname{cis}-\beta-\mathrm{A}$ 0.0

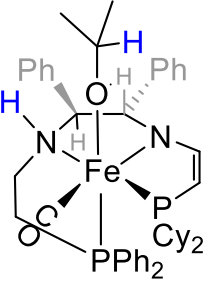

$(S, S)-5$ : $\Delta$-cis- $\beta-\mathrm{A}$ $-5.7$

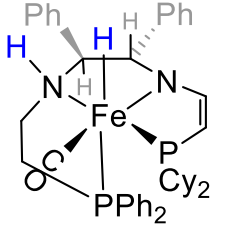
(S,S)-3: $\Delta$-cis- $\beta-A$ $-13.3$

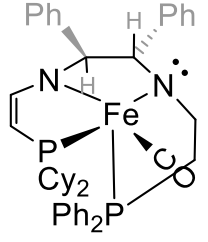

$\Lambda$-cis- $\beta$-A 6.9<smiles>CC(C)O</smiles>

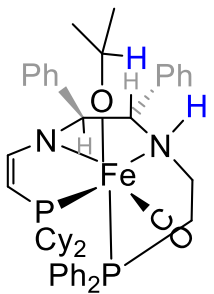

$\Lambda$-cis- $\beta-\mathbf{A}$ $-5.8$<smiles>[3H]C(C)=O</smiles>

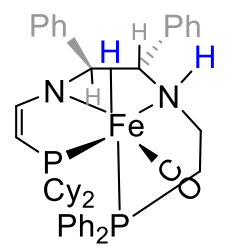

$\Lambda$-cis- $\beta$-A $-10.0$

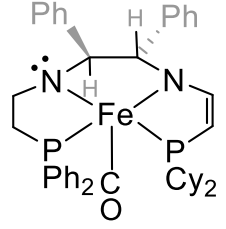

trans

$-3.2$

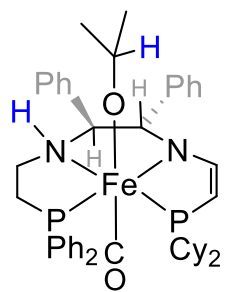

trans- $R_{\mathrm{NH}}$

$-8.3$

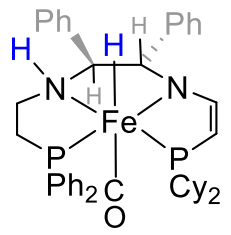

trans-(S,S)-3'

$-8.9$ 
Scheme S3 - Relative energies of possible amido and hydride isomers derived from C1.

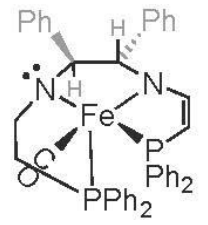

cis $-\beta$ isomers

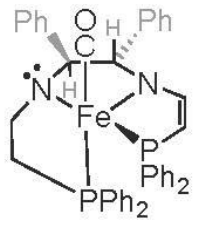

$\Delta-(S, S)-6 B$

17.3

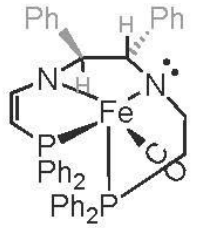

$\Lambda-(S, S)-6 A$

6.6

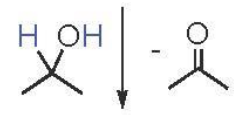

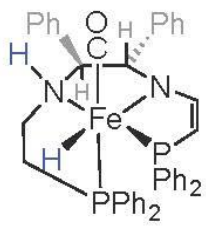

$\Delta-(S, S)-G 1 B$ $-9.9$

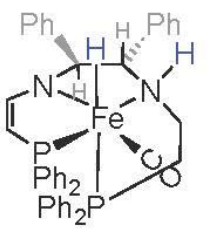

$\Lambda-(S, S)-G 1 A$ $-10.4$ $-10.4$

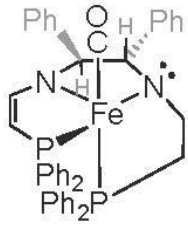

$\Lambda-(S, S)-6 B$

19.3

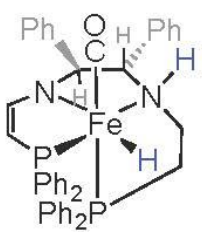

$\Lambda-(S, S)-\mathbf{G 1 B}$

$-6.3$

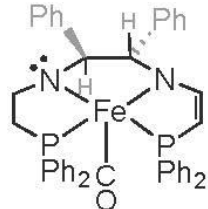

trans-(S,S)-6 $-4.4$ isomers: $-13.6$

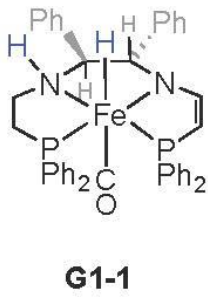

$-10.3$ 
Free Energies ( $G^{\circ}$, Hartree), Enthalpies $\left(H^{\circ}\right.$, Hartree) and Cartesian Coordinates $(\AA)$ of Optimized Structures

\begin{tabular}{|c|c|c|c|}
\hline \\
\hline \multicolumn{4}{|c|}{$\begin{array}{l}\text { acetone } \\
H^{\circ}=-193.115521\end{array}$} \\
\hline \multicolumn{4}{|c|}{$G^{\circ}=-193.149197$} \\
\hline c & 0.00000 & 0.17857 & 0.00000 \\
\hline$C$ & -1.27170 & -0.60888 & -0.00329 \\
\hline $\mathrm{H}$ & -2.13449 & 0.03240 & -0.16508 \\
\hline t & -1.24103 & -1.39249 & -0.76166 \\
\hline $\mathrm{H}$ & -1.38181 & -1.11751 & 0.95723 \\
\hline C & 1.27170 & -0.60888 & 0.00329 \\
\hline $\mathrm{H}$ & 1.24103 & -1.39249 & 0.76166 \\
\hline $\mathrm{H}$ & 1.38181 & -1.11751 & -0.95723 \\
\hline $\mathrm{H}$ & 2.13449 & 0.03240 & 0.16508 \\
\hline 0 & -0.00000 & 1.39880 & 0.00000 \\
\hline
\end{tabular}

\begin{tabular}{|c|c|c|c|}
\hline$H$ & -2.63352 & 0.84690 & -0.39929 \\
\hline C & -2.45920 & -1.05698 & 0.52656 \\
\hline & -2.55127 & -2.11034 & 0.23670 \\
\hline & -1.70104 & 0.52298 & -2.81470 \\
\hline$r$ & -2.68651 & 0.58405 & -3.28595 \\
\hline & -0.99886 & 0.16537 & -3.56713 \\
\hline 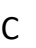 & -1.28495 & 1.87543 & -2.27333 \\
\hline r & -2.14536 & 2.42118 & -1.88970 \\
\hline ト & -0.85146 & 2.49113 & -3.05878 \\
\hline C & -0.57158 & -1.06747 & 2.00755 \\
\hline r & -1.25231 & -1.28218 & 2.83306 \\
\hline$C$ & 0.87802 & -1.06050 & 2.28381 \\
\hline r & 1.14230 & -2.00678 & 2.76681 \\
\hline r & 1.11754 & -0.29482 & 3.02598 \\
\hline C & 1.18663 & -0.66554 & -2.21097 \\
\hline c & -4.16170 & -0.36694 & -1.21828 \\
\hline C & -4.61645 & -1.60057 & -1.68227 \\
\hline r & -3.95222 & -2.46009 & -1.68615 \\
\hline C & -5.91821 & -1.74242 & -2.13718 \\
\hline $\mathrm{H}$ & -6.26131 & -2.70579 & -2 \\
\hline C & -6.78179 & -0.65457 & -2.13054 \\
\hline $\mathrm{H}$ & -7.79939 & -0.76762 & -2.48381 \\
\hline c & -6.33764 & 0.57671 & -1.67075 \\
\hline r & -7.00688 & 1.42832 & -1.66400 \\
\hline C & -5.03323 & 0.71859 & -1.21873 \\
\hline $\mathrm{H}$ & -4.68298 & 1.67963 & -0.85485 \\
\hline C & -3.40188 & -0.76965 & 1.66300 \\
\hline C & -4.23978 & -1.76585 & 2.15294 \\
\hline $\mathrm{H}$ & -4.19114 & -2.76080 & 1.72377 \\
\hline C & -5.13562 & -1.49036 & 3.17780 \\
\hline $\mathrm{H}$ & -5.78521 & -2.27327 & 3.54979 \\
\hline C & -5.1 & -0.21697 & 3.72453 \\
\hline $\mathrm{H}$ & -5.89483 & -0.00252 & 4.52488 \\
\hline C & -4.36080 & 0.78296 & 3.24293 \\
\hline $\mathrm{H}$ & -4.40381 & 1.78062 & 3.66386 \\
\hline C & -3.47119 & 0.50818 & 2.21722 \\
\hline $\mathrm{H}$ & -2.82202 & 1.29139 & 1.83977 \\
\hline C & 2.81720 & -2.46966 & 0.68405 \\
\hline $\mathrm{H}$ & 2.01450 & -3.19801 & 0.86149 \\
\hline C & -0.16875 & 2.26779 & 1.81156 \\
\hline $\mathrm{H}$ & 0.65312 & 1.56742 & 1.88795 \\
\hline C & -0.63358 & 2.89285 & 2.95784 \\
\hline $\mathrm{H}$ & -0.17846 & 2.66600 & 3.91459 \\
\hline C & -1.68155 & 3.79911 & 2.87401 \\
\hline $\mathrm{H}$ & -2.05606 & 4.28283 & 3.76769 \\
\hline C & -2.24478 & 4.08673 & 1.63880 \\
\hline $\mathrm{H}$ & -3.05502 & 4.80155 & 1.56417 \\
\hline C & -1.77283 & 3.46759 & 0.48891 \\
\hline $\mathrm{H}$ & -2.21974 & 3.72652 & -0.46246 \\
\hline C & -0.73430 & 2.53935 & 0.56510 \\
\hline C & 1.32862 & 2.77922 & -1.42772 \\
\hline C & 1.89720 & 2.58776 & -2.6887 \\
\hline $\mathrm{H}$ & 1.54513 & 1.79059 & -3.3329 \\
\hline
\end{tabular}

$\begin{array}{lrrr}\text { isopropanol } & & \\ H^{\circ}= & -194.291128 & & \\ G^{\circ}= & -194.324792 & & \\ C & 0.00260 & 0.03438 & 0.36276 \\ H & -0.01064 & 0.08668 & 1.46070 \\ C & 1.33435 & -0.51028 & -0.08819 \\ H & 2.15412 & 0.12418 & 0.24946 \\ H & 1.49682 & -1.51151 & 0.30996 \\ H & 1.37657 & -0.56879 & -1.17775 \\ C & -1.16167 & -0.81100 & -0.10246 \\ H & -1.10031 & -1.82070 & 0.30624 \\ H & -2.11275 & -0.38249 & 0.21842 \\ H & -1.17376 & -0.88550 & -1.19179 \\ \text { O } & -0.09186 & 1.36929 & -0.16410 \\ H & -0.94685 & 1.72521 & 0.10490\end{array}$

$\Delta$-cis- $\beta-C 4-R_{N H}$

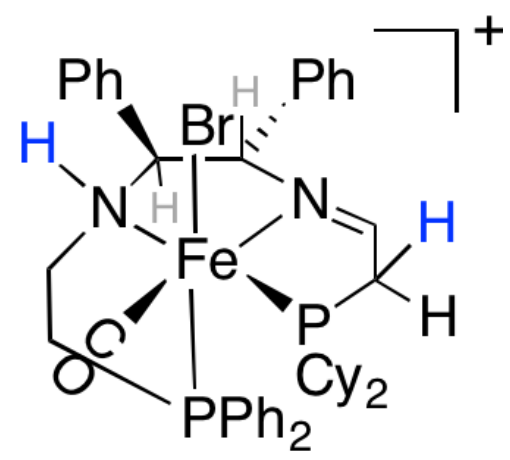

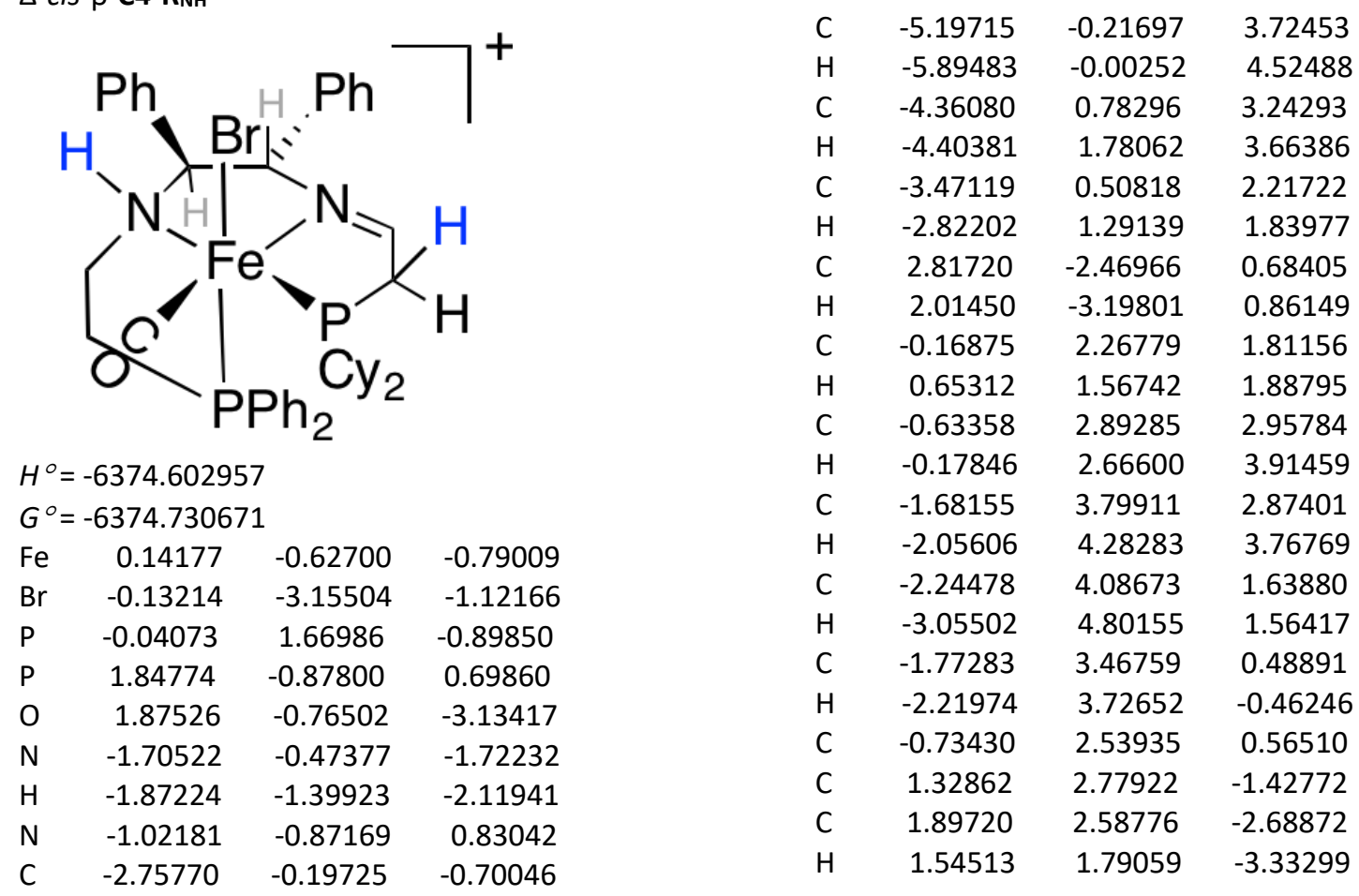




\begin{tabular}{|c|c|c|c|}
\hline C & 2.89637 & 3.43391 & -3.14291 \\
\hline $\mathrm{H}$ & 3.32500 & 3.27694 & -4.12507 \\
\hline C & 3.33616 & 4.48465 & -2.34839 \\
\hline $\mathrm{H}$ & 4.11257 & 5.14874 & -2.70755 \\
\hline C & 2.77520 & 4.68199 & -1.09562 \\
\hline $\mathrm{H}$ & 3.11134 & 5.50011 & -0.47016 \\
\hline C & 1.78065 & 3.83178 & -0.63210 \\
\hline $\mathrm{H}$ & 1.35142 & 4.00050 & 0.34816 \\
\hline C & 3.83470 & -2.60263 & 1.81767 \\
\hline $\mathrm{H}$ & 3.38422 & -2.35285 & 2.78195 \\
\hline $\mathrm{H}$ & 4.65163 & -1.89053 & 1.66211 \\
\hline C & 4.40727 & -4.01441 & 1.85359 \\
\hline $\mathrm{H}$ & 5.14633 & -4.09285 & 2.65445 \\
\hline $\mathrm{H}$ & 3.60604 & -4.71998 & 2.10112 \\
\hline . & 5.02236 & -4.39764 & 0.51665 \\
\hline $\mathrm{H}$ & 5.88996 & -3.75601 & 0.32257 \\
\hline $\mathrm{H}$ & 5.40005 & -5.42218 & 0.55159 \\
\hline$c$ & 4.01948 & -4.23810 & -0.61536 \\
\hline $\mathrm{H}$ & 3.19829 & -4.95159 & -0.47809 \\
\hline $\mathrm{H}$ & 4.48125 & -4.48198 & -1.57496 \\
\hline C & 3.44574 & -2.82761 & -0.66216 \\
\hline $\mathrm{H}$ & 2.69856 & -2.75699 & -1.45322 \\
\hline $\mathrm{H}$ & 4.24441 & -2.12276 & -0.90784 \\
\hline$C$ & 3.02186 & 0.55664 & 0.91814 \\
\hline $\mathrm{H}$ & 2.41269 & 1.42317 & 0.63472 \\
\hline C & 3.53919 & 0.83211 & 2.32942 \\
\hline $\mathrm{H}$ & 2.70926 & 0.92771 & 3.03382 \\
\hline $\mathrm{H}$ & 4.15381 & -0.00103 & 2.68031 \\
\hline C & 4.36193 & 2.11511 & 2.34057 \\
\hline $\mathrm{H}$ & 3.70651 & 2.95949 & 2.09087 \\
\hline $\mathrm{H}$ & 4.73724 & 2.30435 & 3.34879 \\
\hline C & 5.50779 & 2.05603 & 1.34256 \\
\hline $\mathrm{H}$ & 6.20959 & 1.27060 & 1.64642 \\
\hline $\mathrm{H}$ & 6.07062 & 2.99218 & 1.35426 \\
\hline C & 4.99793 & 1.76214 & -0.05922 \\
\hline $\mathrm{H}$ & 5.82825 & 1.68153 & -0.76478 \\
\hline $\mathrm{H}$ & 4.37992 & 2.59796 & -0.40268 \\
\hline C & 4.16909 & 0.48649 & -0.09255 \\
\hline $\mathrm{H}$ & 3.78019 & 0.31067 & -1.10037 \\
\hline 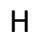 & 4.81376 & -0.36447 & $0.1474 \mathrm{C}$ \\
\hline
\end{tabular}

$\Lambda$-cis- $\beta-$ C4-SNH $_{\mathrm{NH}}$

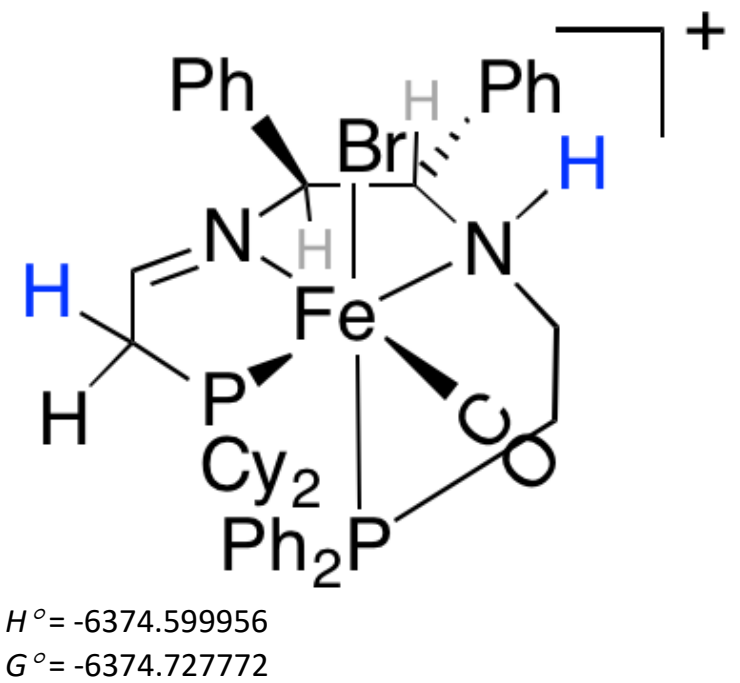

\begin{tabular}{|c|c|c|c|}
\hline - & 0.14177 & -0.62700 & -0.79009 \\
\hline$r$ & -0.13214 & -3.15504 & -112166 \\
\hline & -0.04073 & 1.66986 & -0.89850 \\
\hline & 1.84774 & -0.87800 & 0.69860 \\
\hline & 1.87526 & -0.76502 & -3.13417 \\
\hline & -1.70522 & -0.47377 & -1.72232 \\
\hline & -1.87224 & -1.39923 & -2.11941 \\
\hline & -1.02181 & -0.87169 & 0.83042 \\
\hline & -2.75770 & -0.19725 & -0.70046 \\
\hline & -2.63352 & 0.84690 & -0.39929 \\
\hline & -2.45920 & -1.05698 & 0.52656 \\
\hline & -2.55127 & -2.11034 & 0.23670 \\
\hline & -1.70104 & 0.52298 & -2.81470 \\
\hline & -2.68651 & 0.58405 & -3.28595 \\
\hline & -0.99886 & 0.16537 & -3.56713 \\
\hline & -1.28495 & 1.87543 & -2.27333 \\
\hline $\mathrm{H}$ & -2.14536 & 2.42118 & -1.88970 \\
\hline & -0.85146 & 2.49113 & -3.05878 \\
\hline & -0.57158 & -1.06747 & 2.00755 \\
\hline H & -1.25231 & -1.28218 & 2.83306 \\
\hline & 0.87802 & -1.06050 & 2.28381 \\
\hline $\mathrm{H}$ & 1.14230 & -2.00678 & 2.76681 \\
\hline & 1.11754 & -0.29482 & 3.02598 \\
\hline & 1.18663 & -0.66554 & -2.21097 \\
\hline & -4.16170 & -0.36694 & -1.21828 \\
\hline & -4.61645 & -1.60057 & -1.68227 \\
\hline $\mathrm{H}$ & -3.95222 & -2.46009 & -1.68615 \\
\hline & -5.91821 & -1.74242 & -2.13718 \\
\hline & -6.26131 & -2.70579 & -2.49409 \\
\hline & -6.78179 & -0.65457 & -2.13054 \\
\hline & -7.79939 & -0.76762 & -2.48381 \\
\hline & -6.33764 & 0.57671 & -1.67075 \\
\hline & -7.00688 & 1.42832 & -1.66400 \\
\hline & -5.03323 & 0.71859 & -1.21873 \\
\hline & -4.68298 & 1.67963 & -0.85485 \\
\hline & -3.40188 & -0.76965 & 1.66300 \\
\hline $\mathrm{C}$ & -4.23978 & -1.76585 & 2.15294 \\
\hline & -4.19114 & -2.76080 & 1.72377 \\
\hline C & -5.13562 & -1.49036 & 3.17780 \\
\hline $\mathrm{H}$ & -5.78521 & -2.27327 & 3.54979 \\
\hline C & -5.19715 & -0.21697 & 3.72453 \\
\hline $\mathrm{H}$ & -5.89483 & -0.00252 & 4.52488 \\
\hline & -4.36080 & 0.78296 & 3.24293 \\
\hline $\mathrm{H}$ & -4.40381 & 1.78062 & 3.66386 \\
\hline C & -3.47119 & 0.50818 & 2.21722 \\
\hline $\mathrm{H}$ & -2.82202 & 1.29139 & 1.83977 \\
\hline C & 2.81720 & -2.46966 & 0.68405 \\
\hline $\mathrm{H}$ & 2.01450 & -3.19801 & 0.86149 \\
\hline C & -0.16875 & 2.26779 & 1.81156 \\
\hline $\mathrm{H}$ & 0.65312 & 1.56742 & 1.88795 \\
\hline & -0.63358 & 2.89285 & 2.95784 \\
\hline H & -0.17846 & 2.66600 & 3.91459 \\
\hline $\mathrm{C}$ & -1.68155 & 3.79911 & 2.87401 \\
\hline $\mathrm{H}$ & -2.05606 & 4.28283 & 3.76769 \\
\hline 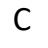 & -2.24478 & 4.08673 & 1.63880 \\
\hline & -3.05502 & 4.80155 & 1.56417 \\
\hline r & -1.77283 & 3.46759 & 0.48891 \\
\hline & -2.21974 & 3.72652 & -0.46246 \\
\hline
\end{tabular}




\begin{tabular}{|c|c|c|c|}
\hline$C$ & -0.73430 & 2.53935 & 0.56510 \\
\hline C & 1.32862 & 2.77922 & -1.42772 \\
\hline C & 1.89720 & 2.58776 & -2.68872 \\
\hline $\mathrm{H}$ & 1.54513 & 1.79059 & -3.33299 \\
\hline 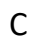 & 2.89637 & 3.43391 & -3.14291 \\
\hline $\mathrm{H}$ & 3.32500 & 3.27694 & -4.12507 \\
\hline C & 3.33616 & 4.48465 & -2.34839 \\
\hline $\mathrm{H}$ & 4.11257 & 5.14874 & -2.70755 \\
\hline C & 2.77520 & 4.68199 & -1.09562 \\
\hline $\mathrm{H}$ & 3.11134 & 5.50011 & -0.47016 \\
\hline C & 1.78065 & 3.83178 & -0.63210 \\
\hline $\mathrm{H}$ & 1.35142 & 4.00050 & 0.34816 \\
\hline$C$ & 3.83470 & -2.60263 & 1.81767 \\
\hline $\mathrm{H}$ & 3.38422 & -2.35285 & 2.78195 \\
\hline $\mathrm{H}$ & 4.65163 & -1.89053 & 1.66211 \\
\hline C & 4.40727 & -4.01441 & 1.85359 \\
\hline $\mathrm{H}$ & 5.14633 & -4.09285 & 2.65445 \\
\hline $\mathrm{H}$ & 3.60604 & -4.71998 & 2.10112 \\
\hline C & 5.02236 & -4.39764 & 0.51665 \\
\hline $\mathrm{H}$ & 5.88996 & -3.75601 & 0.32257 \\
\hline $\mathrm{H}$ & 5.40005 & -5.4 & 0.55159 \\
\hline C & 4.01948 & -4.23810 & -0.61536 \\
\hline $\mathrm{H}$ & 3.19829 & -4.95159 & -0.47809 \\
\hline $\mathrm{H}$ & 4.48125 & -4.48198 & -1.57496 \\
\hline C & 3.44574 & -2.82761 & -0.66216 \\
\hline $\mathrm{H}$ & 2.69856 & -2.75699 & -1.45322 \\
\hline $\mathrm{H}$ & 4.24441 & -2.12276 & -0.90784 \\
\hline C & 3.02186 & 0.55664 & 0.91814 \\
\hline $\mathrm{H}$ & 2.41269 & 1.42317 & 0.63472 \\
\hline C & 3.53919 & 0.83211 & 2.32942 \\
\hline $\mathrm{H}$ & 2.70926 & 0.92771 & 3.03382 \\
\hline $\mathrm{H}$ & 4.15381 & -0.00103 & 2.68031 \\
\hline C & 4.36193 & 2.11511 & 2.34057 \\
\hline $\mathrm{H}$ & 3.70651 & 2.95949 & 2.09087 \\
\hline $\mathrm{H}$ & 4.73724 & 2.30435 & 3.34879 \\
\hline C & 5.50779 & 2.05603 & 1.34256 \\
\hline $\mathrm{H}$ & 6.20959 & 1.27060 & 1.64642 \\
\hline $\mathrm{H}$ & 6.07062 & 2.99218 & 1.35426 \\
\hline C & 4.99793 & 1.76214 & -0.05922 \\
\hline $\mathrm{H}$ & 5.82825 & 1.68153 & -0.76478 \\
\hline $\mathrm{H}$ & 4.37992 & 2.59796 & -0.40268 \\
\hline C & 4.16909 & 0.48649 & -0.09255 \\
\hline $\mathrm{H}$ & 3.78019 & 0.31067 & -1.10037 \\
\hline $\mathrm{H}$ & 4.81376 & -0.36447 & 0.14749 \\
\hline
\end{tabular}

$\Delta-(S, S)-4 A$

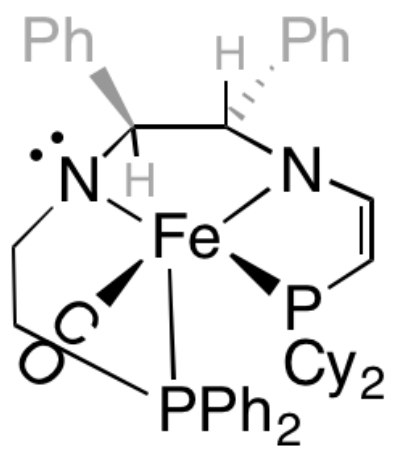

$H^{\circ}=-3799.390461$

\begin{tabular}{|c|c|c|c|}
\hline \multicolumn{4}{|c|}{$G^{\circ}=-3799.515843$} \\
\hline $\mathrm{Fe}$ & 0.08503 & -0.60415 & -0.89362 \\
\hline 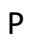 & -0.05776 & 1.58153 & -0.80385 \\
\hline 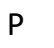 & 1.77231 & -1.20426 & 0.52599 \\
\hline & 1.81833 & -0.53690 & -3.23682 \\
\hline$\sqrt{ }$ & -1.63366 & -0.48089 & -1.80327 \\
\hline J & -1.02945 & -1.18679 & 0.58621 \\
\hline & -2.72524 & -0.35257 & -0.83843 \\
\hline $\mathrm{H}$ & -2.72188 & 0.64372 & -0.35367 \\
\hline & -2.44780 & -1.35679 & 0.28450 \\
\hline $\mathrm{H}$ & -2.61498 & -2.36445 & -0.13039 \\
\hline$C$ & -1.67156 & 0.60467 & -2.77924 \\
\hline-1 & -2.65760 & 0.68265 & -3.25799 \\
\hline $\mathrm{H}$ & -0.95793 & 0.37636 & -3.57470 \\
\hline C & -1.31520 & 1.94513 & -2.13808 \\
\hline $\mathrm{H}$ & -2.19031 & 2.40361 & -1.67674 \\
\hline-1 & -0.91883 & 2.67157 & -2.84850 \\
\hline$C$ & -0.51504 & -1.56390 & 1.77422 \\
\hline $\mathrm{H}$ & -1.20695 & -1.89177 & 2.55255 \\
\hline C & 0.82726 & -1.52866 & 2.01569 \\
\hline $\mathrm{H}$ & 1.24688 & -1.83824 & 2.96393 \\
\hline[ & 1.12355 & -0.52673 & -2.29827 \\
\hline$c$ & -4.09835 & -0.53704 & -1.43794 \\
\hline C & -4.39952 & -1.65922 & -2.20925 \\
\hline $\mathrm{H}$ & -3.62079 & -2.38672 & -2.41153 \\
\hline C & -5.67530 & -1.84582 & -2.71985 \\
\hline $\mathrm{H}$ & -5.89481 & -2.72402 & -3.31600 \\
\hline$C$ & -6.67276 & -0.91113 & -2.46838 \\
\hline $\mathrm{H}$ & -7.66918 & -1.05707 & -2.86802 \\
\hline C & -6.38369 & 0.21228 & -1.70661 \\
\hline $\mathrm{H}$ & -7.15461 & 802 & -1.50954 \\
\hline C & -5.10426 & 0.39580 & -1.19780 \\
\hline $\mathrm{H}$ & -4.87703 & 1.27331 & -0.59823 \\
\hline C & -3.40495 & -1.16661 & 1.43462 \\
\hline C & -4.40905 & -2.09827 & 1.68275 \\
\hline $\mathrm{H}$ & -4.45985 & -2.99609 & 1.07489 \\
\hline C & -5.34499 & -1.88556 & 2.68740 \\
\hline $\mathrm{H}$ & -6.12169 & -2.62003 & 2.86499 \\
\hline C & -5.28547 & -0.73598 & 3.46192 \\
\hline $\mathrm{H}$ & -6.01353 & -0.56907 & 4.24664 \\
\hline C & -4.28313 & 0.19834 & 3.22755 \\
\hline $\mathrm{H}$ & -4.22723 & 1.09925 & 3.82797 \\
\hline C & -3.35357 & -0.01613 & 2.22204 \\
\hline $\mathrm{H}$ & -2.57252 & 0.71407 & 2.03901 \\
\hline C & 2.57866 & -2.86939 & 0.19178 \\
\hline $\mathrm{H}$ & 1.71731 & -3.53868 & 0.32853 \\
\hline C & -0.13850 & 1.85557 & 1.96277 \\
\hline $\mathrm{H}$ & 0.64746 & 1.10910 & 1.94648 \\
\hline C & -0.58104 & 2.35384 & 3.17873 \\
\hline $\mathrm{H}$ & -0.12461 & 2.00679 & 4.09838 \\
\hline C & -1.61398 & 3.28091 & 3.21449 \\
\hline $\mathrm{H}$ & -1.96868 & 3.66501 & 4.16326 \\
\hline C & -2.19219 & 3.71288 & 2.02888 \\
\hline $\mathrm{H}$ & -2.99591 & 4.43908 & 2.04911 \\
\hline C & -1.74284 & 3.22274 & 0.80980 \\
\hline $\mathrm{H}$ & -2.20003 & 3.58608 & -0.10229 \\
\hline C & -0.71526 & 2.28112 & 0.76504 \\
\hline C & 1.34731 & 2.70852 & -1.18602 \\
\hline
\end{tabular}




\begin{tabular}{|c|c|c|c|}
\hline$C$ & 1.86841 & 2.71989 & -2.48256 \\
\hline $\mathrm{H}$ & 1.44333 & 2.07959 & -3.24704 \\
\hline C & 2.91990 & 3.56110 & -2.81172 \\
\hline $\mathrm{H}$ & 3.30883 & 3.56119 & -3.82273 \\
\hline$C$ & 3.46489 & 4.40641 & -1.85394 \\
\hline $\mathrm{H}$ & 4.28368 & 5.06612 & -2.11388 \\
\hline$c$ & 2.95458 & 4.40231 & -0.56432 \\
\hline $\mathrm{H}$ & 3.37263 & 5.05884 & 0.18946 \\
\hline$c$ & 1.90662 & 3.55582 & -0.22909 \\
\hline $\mathrm{H}$ & 1.51900 & 3.56825 & 0.78264 \\
\hline C & 3.63730 & -3.26575 & 1.21957 \\
\hline $\mathrm{H}$ & 3.24641 & -3.14739 & 2.23366 \\
\hline $\mathrm{H}$ & 4.49613 & -2.59151 & 1.13467 \\
\hline$C$ & 4.11500 & -4.69503 & 0.99833 \\
\hline $\mathrm{H}$ & 4.88597 & -4.94764 & 1.73070 \\
\hline $\mathrm{H}$ & 3.28257 & -5.38599 & 1.17508 \\
\hline$C$ & 4.63566 & -4.88535 & -0.41738 \\
\hline $\mathrm{H}$ & 5.52399 & -4.25870 & -0.56159 \\
\hline $\mathrm{H}$ & 4.96018 & -5.91731 & -0.57131 \\
\hline$C$ & 3.57840 & -4.50261 & -1.44079 \\
\hline $\mathrm{H}$ & 2.73048 & -5.19197 & -1.35416 \\
\hline $\mathrm{H}$ & 3.96521 & -4.61874 & -2.45623 \\
\hline$C$ & 3.08041 & -3.07669 & -1.23659 \\
\hline $\mathrm{H}$ & 2.27789 & -2.86069 & -1.94432 \\
\hline $\mathrm{H}$ & 3.89010 & -2.37504 & -1.46063 \\
\hline C & 3.09375 & 0.06211 & 0.88962 \\
\hline $\mathrm{H}$ & 2.53819 & 1.00400 & 0.78127 \\
\hline C & 3.66735 & 0.05299 & 2.30515 \\
\hline $\mathrm{H}$ & 2.85645 & 0.07333 & 3.03837 \\
\hline $\mathrm{H}$ & 4.22445 & -0.87154 & 2.48356 \\
\hline C & 4.59357 & 1.24612 & 2.50653 \\
\hline $\mathrm{H}$ & 4.00677 & 2.17083 & 2.42989 \\
\hline $\mathrm{H}$ & 5.01161 & 1.23058 & 3.51601 \\
\hline C & 5.70402 & 1.26841 & 1.46676 \\
\hline $\mathrm{H}$ & 6.34184 & 0.38713 & 1.60433 \\
\hline $\mathrm{H}$ & 6.34818 & 2.13858 & 1.61443 \\
\hline C & 5.13691 & 1.26298 & 0.05539 \\
\hline $\mathrm{H}$ & 5.94165 & 1.24414 & -0.68394 \\
\hline $\mathrm{H}$ & 4.58371 & 2.19307 & -0.11485 \\
\hline C & 4.19816 & 0.08471 & -0.16560 \\
\hline $\mathrm{H}$ & 3.76382 & 0.13049 & -1.16920 \\
\hline 4 & 4.77222 & -0.84666 & -0.1162 \\
\hline
\end{tabular}

\begin{tabular}{|c|c|c|c|}
\hline$P$ & 0.02067 & -1.55080 & -0.95916 \\
\hline$P$ & -1.87502 & 1.13737 & 0.51403 \\
\hline & 0.07132 & 3.75176 & -1.28050 \\
\hline | & 1.67698 & 0.63918 & -1.85436 \\
\hline $\mathrm{N}$ & 1.01172 & 0.97549 & 0.67415 \\
\hline & 2.70126 & 0.36452 & -0.86157 \\
\hline P & 2.64110 & -0.66867 & -0.46098 \\
\hline 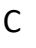 & 2.40607 & 1.25441 & 0.35980 \\
\hline H & 2.52337 & 2.30510 & 0.04030 \\
\hline C & 1.59786 & -0.39643 & -2.86321 \\
\hline F & 2.52633 & -0.45909 & -3.45302 \\
\hline $\mathrm{H}$ & 0.81499 & -0.11566 & -3.57625 \\
\hline 0 & 1.28057 & -1.79167 & -2.30133 \\
\hline † & 2.17524 & -2.25068 & -1.87957 \\
\hline $\mathrm{H}$ & 0.89810 & -2.47249 & -3.06203 \\
\hline C & 0.44765 & 1.35419 & 1.82613 \\
\hline $\mathrm{H}$ & 1.11225 & 1.57872 & 2.66398 \\
\hline$C$ & -0.91150 & 1.41857 & 1.99550 \\
\hline $\mathrm{H}$ & -1.33976 & 1.66745 & 2.95860 \\
\hline C & -0.02480 & 2.60315 & -1.13244 \\
\hline C & 4.11071 & 0.54142 & -1.37597 \\
\hline C & 4.48842 & 1.71108 & -2.03434 \\
\hline$r$ & 3.74079 & 2.47695 & -2.21333 \\
\hline 0 & 5.79588 & 1.89533 & -2.45885 \\
\hline H & 6.07588 & 2.81247 & -2.96434 \\
\hline 0 & 6.74854 & 0.90735 & -2.23719 \\
\hline $\mathrm{H}$ & 7.76950 & 1.05042 & -2.57067 \\
\hline C & 6.38267 & -0.26577 & -1.59200 \\
\hline$\vdash$ & 7.11772 & -1.04386 & -1.42176 \\
\hline C & 5.07257 & -0.44376 & -1.16547 \\
\hline$\vdash$ & 4.78580 & -1.35898 & -0.65389 \\
\hline C & 3.37 & 0.98797 & 1.48533 \\
\hline$C$ & 4.34807 & 1.92081 & 1.82397 \\
\hline H & 4.37079 & 2.87463 & 1.30672 \\
\hline C & 5.29167 & 1.63845 & 2.80391 \\
\hline H & 6.04704 & 2.37444 & 3.05299 \\
\hline$C$ & 5.26625 & 0.41803 & 3.46372 \\
\hline H & 5.99994 & 0.19726 & 4.22975 \\
\hline C & 4.29108 & -0.51800 & 3.13917 \\
\hline H & 4.26354 & -1.47280 & 3.65200 \\
\hline C & 3.35436 & -0.23492 & 2.15734 \\
\hline H & 2.59430 & -0.96591 & 1.90142 \\
\hline C & -2.88975 & 2.69394 & 0.29746 \\
\hline $\mathrm{H}$ & -2.11001 & 3.45886 & 0.42001 \\
\hline C & 0.10401 & -2.05339 & 1.77024 \\
\hline $\mathrm{H}$ & -0.62310 & -1.25117 & 1.83058 \\
\hline C & 0.51833 & -2.68803 & 2.93174 \\
\hline $\mathrm{H}$ & 0.09453 & -2.39217 & 3.88442 \\
\hline C & 1.48525 & -3.68258 & 2.87344 \\
\hline $\mathrm{H}$ & 1.81684 & -4.17361 & 3.78034 \\
\hline C & 2.03014 & -4.04282 & 1.64831 \\
\hline $\mathrm{H}$ & 2.78371 & -4.81943 & 1.59597 \\
\hline C & 1.60699 & -3.41784 & 0.48306 \\
\hline $\mathrm{H}$ & 2.03065 & -3.72505 & -0.46579 \\
\hline C & 0.63998 & -2.41370 & 0.53285 \\
\hline C & -1.39073 & -2.59429 & -1.51104 \\
\hline C & -2.14622 & -2.13995 & -2.59510 \\
\hline 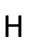 & -1.90672 & -1.18628 & -3.05705 \\
\hline
\end{tabular}

\section{$\Delta-(S, S)-4 B$}

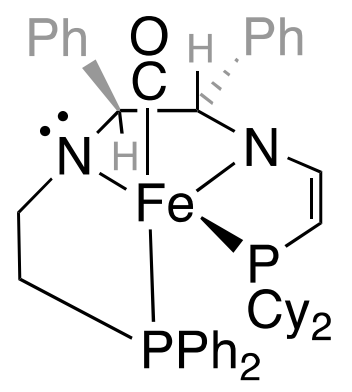

$H^{\circ}=-3799.366002$

$G^{\circ}=-3799.492309$

$\begin{array}{llll}\mathrm{Fe} & -0.08897 & 0.87641 & -0.91530\end{array}$ 


\begin{tabular}{llll}
$\mathrm{C}$ & -3.19643 & -2.89774 & -3.08966 \\
$\mathrm{H}$ & -3.77135 & -2.53436 & -3.93299 \\
$\mathrm{C}$ & -3.51285 & -4.11615 & -2.50191 \\
$\mathrm{H}$ & -4.33437 & -4.70765 & -2.88708 \\
$\mathrm{C}$ & -2.77820 & -4.56761 & -1.41485 \\
$\mathrm{H}$ & -3.02433 & -5.51375 & -0.94790 \\
$\mathrm{C}$ & -1.72499 & -3.81108 & -0.91753 \\
$\mathrm{H}$ & -1.15809 & -4.17778 & -0.06946 \\
$\mathrm{C}$ & -3.94589 & 2.96104 & 1.36655 \\
$\mathrm{H}$ & -3.51517 & 2.85214 & 2.36555 \\
$\mathrm{H}$ & -4.74663 & 2.21756 & 1.28897 \\
$\mathrm{C}$ & -4.54094 & 4.35434 & 1.19735 \\
$\mathrm{H}$ & -5.31533 & 4.52585 & 1.94937 \\
$\mathrm{H}$ & -3.75969 & 5.10026 & 1.38377 \\
$\mathrm{C}$ & -5.10335 & 4.55492 & -0.20212 \\
$\mathrm{H}$ & -5.95519 & 3.87969 & -0.34717 \\
$\mathrm{H}$ & -5.49483 & 5.56901 & -0.31347 \\
$\mathrm{C}$ & -4.05568 & 4.26876 & -1.26847 \\
$\mathrm{H}$ & -3.24702 & 5.00576 & -1.19259 \\
$\mathrm{H}$ & -4.48491 & 4.38433 & -2.26691 \\
$\mathrm{C}$ & -3.47106 & 2.87225 & -1.10504 \\
$\mathrm{H}$ & -2.69990 & 2.68723 & -1.85961 \\
$\mathrm{H}$ & -4.25781 & 2.13278 & -1.28124 \\
$\mathrm{C}$ & -3.04960 & -0.28604 & 0.83513 \\
$\mathrm{H}$ & -2.41792 & -1.15901 & 0.62087 \\
$\mathrm{C}$ & -3.52890 & -0.44238 & 2.27584 \\
$\mathrm{H}$ & -2.67641 & -0.41238 & 2.96017 \\
$\mathrm{H}$ & -4.17794 & 0.39565 & 2.55030 \\
$\mathrm{C}$ & -4.28815 & -1.75322 & 2.43805 \\
$\mathrm{H}$ & -3.59390 & -2.58590 & 2.26431 \\
$\mathrm{H}$ & -4.64401 & -1.85908 & 3.46592 \\
$\mathrm{C}$ & -5.44735 & -1.85454 & 1.45718 \\
$\mathrm{H}$ & -6.18909 & -1.08333 & 1.69670 \\
$\mathrm{H}$ & -5.95766 & -2.81460 & 1.56747 \\
-4.97389 & -1.66643 & 0.02358 \\
$\mathrm{H}$ & -5.81667 & -1.70998 & -0.67128 \\
$\mathrm{H}$ & -4.21895 & -0.35481 & -0.14391 \\
$\mathrm{H}$ & -2.8699 & -0.24784 & -1.17405 \\
& 0.48158 & 0.03824 \\
\hline
\end{tabular}

\begin{tabular}{|c|c|c|c|}
\hline O & 1.50690 & 0.13864 & -3.37977 \\
\hline $\mathrm{N}$ & -1.73639 & -0.31783 & -1.67353 \\
\hline$N$ & -1.12868 & 0.88106 & 0.57354 \\
\hline 0 & -2.81075 & 0.43822 & -1.00748 \\
\hline H & -2.70389 & 1.48165 & -1.33587 \\
\hline C & -2.53532 & 0.47428 & 0.50355 \\
\hline $\mathrm{H}$ & -2.63774 & -0.54380 & 0.91644 \\
\hline C & -2.03420 & -1.74113 & -1.78798 \\
\hline $\mathrm{H}$ & -2.66117 & -1.95899 & -2.66712 \\
\hline $\mathrm{H}$ & -2.60772 & -2.11855 & -0.92553 \\
\hline C & -0.75021 & -2.53588 & -1.86650 \\
\hline $\mathrm{H}$ & -0.21704 & -2.34265 & -2.80043 \\
\hline $\mathrm{H}$ & -0.90198 & -3.61411 & -1.79026 \\
\hline C & -0.62513 & 1.40826 & 1.71016 \\
\hline$H$ & -1.31681 & 1.62844 & 2.52602 \\
\hline$C$ & 0.70372 & 1.67817 & 1.84649 \\
\hline $\mathrm{H}$ & 1.10154 & 2.17552 & 2.72132 \\
\hline C & 0.93680 & 0.17643 & -2.36144 \\
\hline C & -4.19805 & 0.00417 & -1.41465 \\
\hline C & -4.72618 & 0.48762 & -2.61231 \\
\hline $\mathrm{H}$ & -4.14081 & 1.18866 & -3.19867 \\
\hline C & -5.97516 & 0.08574 & -3.06122 \\
\hline $\mathrm{H}$ & -6.36472 & 0.47550 & -3.99446 \\
\hline C & -6.72719 & -0.81102 & -2.31354 \\
\hline $\mathrm{H}$ & -7.70506 & -1.12491 & -2.65865 \\
\hline C & -6.21789 & -1.29877 & -1.11831 \\
\hline $\mathrm{H}$ & -6.79787 & -1.99742 & -0.52660 \\
\hline C & -4.96491 & -0.89665 & -0.67508 \\
\hline $\mathrm{H}$ & -4.58049 & -1.29502 & 0.25754 \\
\hline C & -3.48155 & 1.37825 & 1.24483 \\
\hline C & -3.56140 & 2.73903 & 0.93814 \\
\hline$H$ & -2.90525 & 3.15481 & 0.18061 \\
\hline C & -4.45690 & 3.56410 & 1.59999 \\
\hline $\mathrm{H}$ & -4.50619 & 4.61699 & 1.34777 \\
\hline C & -5.28761 & 3.04696 & 2.58746 \\
\hline $\mathrm{H}$ & -5.98613 & 3.69302 & 3.10548 \\
\hline C & -5.20982 & 1.69976 & 2.91006 \\
\hline $\mathrm{H}$ & -5.84715 & 1.28885 & 3.68431 \\
\hline C & -4.31171 & 0.87549 & 2.24466 \\
\hline $\mathrm{H}$ & -4.24868 & -0.17778 & 2.50133 \\
\hline C & -0.45076 & -2.42959 & 1.04513 \\
\hline C & -0.08075 & -1.78601 & 2.22664 \\
\hline $\mathrm{H}$ & 0.65927 & -0.99252 & 2.19647 \\
\hline C & -0.66874 & -2.13350 & 3.43233 \\
\hline $\mathrm{H}$ & -0.37471 & -1.62254 & 4.34157 \\
\hline C & -1.63866 & -3.12768 & 3.47031 \\
\hline $\mathrm{H}$ & -2.10827 & -3.39268 & 4.40987 \\
\hline C & -2.00271 & -3.78301 & 2.30274 \\
\hline $\mathrm{H}$ & -2.75266 & -4.56445 & 2.32905 \\
\hline C & -1.40861 & -3.44227 & 1.09428 \\
\hline $\mathrm{H}$ & -1.69798 & -3.96968 & 0.19379 \\
\hline C & 1.87422 & -2.78979 & -0.64485 \\
\hline C & 2.56078 & -2.80271 & -1.86167 \\
\hline $\mathrm{H}$ & 2.18894 & -2.23168 & -2.70420 \\
\hline C & 3.71245 & -3.56018 & -2.01074 \\
\hline $\mathrm{H}$ & 4.22938 & -3.56678 & -2.96267 \\
\hline C & 4.19820 & -4.31059 & -0.94786 \\
\hline 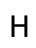 & 5.09629 & -4.90413 & -1.06736 \\
\hline
\end{tabular}

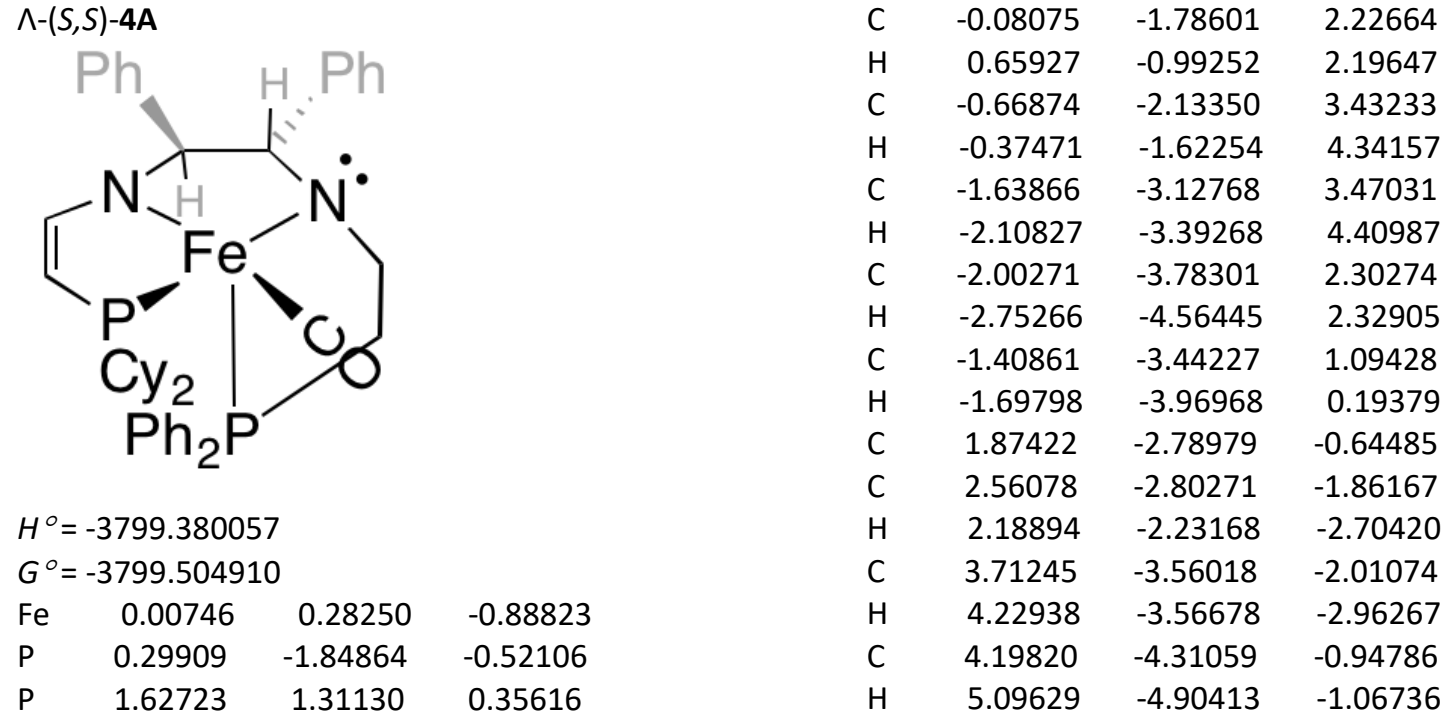




\begin{tabular}{|c|c|c|c|}
\hline$C$ & 3.53138 & -4.29195 & 0.26849 \\
\hline $\mathrm{H}$ & 3.90634 & -4.87030 & 1.10431 \\
\hline C & 2.37822 & -3.53451 & 0.42228 \\
\hline $\mathrm{H}$ & 1.86371 & -3.53579 & 1.37599 \\
\hline C & 2.18882 & 2.99856 & -0.24670 \\
\hline $\mathrm{H}$ & 1.25261 & 3.56785 & -0.15542 \\
\hline C & 3.22771 & 3.67413 & 0.64637 \\
\hline $\mathrm{H}$ & 2.90225 & 3.65895 & 1.68987 \\
\hline $\mathrm{H}$ & 4.16545 & 3.10979 & 0.60227 \\
\hline C & 3.49708 & 5.10361 & 0.19472 \\
\hline $\mathrm{H}$ & 2.58495 & 5.69890 & 0.31802 \\
\hline $\mathrm{H}$ & 4.25347 & 5.56140 & 0.83716 \\
\hline C & 3.93230 & 5.14451 & -1.26138 \\
\hline $\mathrm{H}$ & 4.89111 & 4.62265 & -1.36516 \\
\hline $\mathrm{H}$ & 4.10780 & 6.17463 & -1.58079 \\
\hline C & 2.89806 & 4.47966 & -2.15565 \\
\hline $\mathrm{H}$ & 1.96952 & 5.06152 & -2.12277 \\
\hline $\mathrm{H}$ & 3.22746 & 4.48729 & -3.19762 \\
\hline C & 2.60173 & 3.05044 & -1.71742 \\
\hline $\mathrm{H}$ & 3.48719 & 2.42843 & -1.88378 \\
\hline $\mathrm{H}$ & 1.80877 & 2.63097 & -2.33894 \\
\hline C & 3.11198 & 0.28997 & 0.83416 \\
\hline $\mathrm{H}$ & 2.67204 & -0.71256 & 0.93163 \\
\hline C & 3.75290 & 0.61124 & 2.18363 \\
\hline $\mathrm{H}$ & 2.99122 & 0.61718 & 2.96727 \\
\hline $\mathrm{H}$ & 4.19323 & 1.61207 & 2.16828 \\
\hline C & 4.83661 & -0.40984 & 2.50894 \\
\hline $\mathrm{H}$ & 5.30156 & -0.16922 & 3.46806 \\
\hline $\mathrm{H}$ & 4.37259 & -1.39695 & 2.63017 \\
\hline C & 5.88406 & -0.48177 & 1.40795 \\
\hline $\mathrm{H}$ & 6.41161 & 0.47774 & 1.34834 \\
\hline $\mathrm{H}$ & 6.64071 & -1.23222 & 1.64957 \\
\hline C & 5.24365 & -0.78941 & 0.06297 \\
\hline $\mathrm{H}$ & 4.79887 & -1.78992 & 0.09736 \\
\hline $\mathrm{H}$ & 5.99685 & -0.81322 & -0.72882 \\
\hline C & 4.15531 & 0.21821 & -0.27853 \\
\hline $\mathrm{H}$ & 3.68015 & -0.04116 & -1.23055 \\
\hline 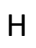 & 4.60806 & 1.20621 & -0.41662 \\
\hline
\end{tabular}

\begin{tabular}{|c|c|c|c|}
\hline $\mathrm{H}$ & -2.69750 & 1.57496 & -1.21785 \\
\hline 0 & -2.49516 & 0.33923 & 0.49788 \\
\hline 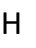 & -2.59628 & -0.73088 & 0.74849 \\
\hline C & -2.00161 & -1.59576 & -1.92804 \\
\hline T & -2.66208 & -1.78305 & -2.78917 \\
\hline & -2.53516 & -2.02135 & -1.0621 \\
\hline C & -0.71582 & -2.36693 & -2.0982 \\
\hline r & -0.19773 & -2.07823 & -3.0168 \\
\hline 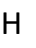 & -0.86224 & -3.44793 & -2.1290 \\
\hline C & -0.56027 & 1.27622 & 1.71340 \\
\hline 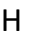 & -1.21425 & 1.44886 & 2.57118 \\
\hline$C$ & 0.77106 & 1.62664 & 1.78933 \\
\hline $\mathrm{H}$ & 1.16985 & 2.09184 & 2.68191 \\
\hline$C$ & -0.34798 & 2.18511 & -1.36080 \\
\hline$C$ & -4.18984 & 0.10416 & -1.43177 \\
\hline C & -4.74234 & 0.70777 & -2.56242 \\
\hline $\mathrm{H}$ & -4.16932 & 1.46859 & -3.08319 \\
\hline C & -5.99941 & 0.35139 & -3.02684 \\
\hline $\mathrm{H}$ & -6.40782 & 0.83599 & -3.90611 \\
\hline C & -6.73602 & -0.62091 & -2.3 \\
\hline $\mathrm{H}$ & -7.71997 & -0.90035 & -2.7198 \\
\hline C & -6.20226 & -1.22961 & -1.2 \\
\hline $\mathrm{H}$ & -6.76904 & -1.98944 & -0.7100 \\
\hline$C$ & -4.94164 & -0.87130 & -0.7765 \\
\hline $\mathrm{H}$ & -4.53973 & -1.36522 & \\
\hline C & -3.43198 & 1.11411 & 1.38345 \\
\hline C & -3.56382 & 2.49802 & 1.24541 \\
\hline $\mathrm{H}$ & -2.95745 & 3.02166 & 0.51285 \\
\hline C & -4.44787 & 3.20809 & 2.04209 \\
\hline $\mathrm{H}$ & -4.54097 & 4.28089 & \\
\hline C & -5.21178 & 2.54897 & 2.99880 \\
\hline $\mathrm{H}$ & -5.90109 & 3.10530 & \\
\hline C & -5.07970 & 1.17685 & 3.15528 \\
\hline $\mathrm{H}$ & -5.66402 & 0.65691 & 3.90537 \\
\hline C & -4.19449 & 0.46793 & 2.35343 \\
\hline $\mathrm{H}$ & -4.08730 & -0.60554 & 2.47832 \\
\hline C & -0.39407 & -2.52620 & 0.80013 \\
\hline C & -0.07552 & -1.93526 & 2.02330 \\
\hline $\mathrm{H}$ & 0.62438 & -1.10581 & 2.05409 \\
\hline C & -0.66579 & -2.38302 & 3.19551 \\
\hline $\mathrm{H}$ & -0.41390 & -1.91207 & 4.13842 \\
\hline C & -1.58576 & -3.42254 & 3.15679 \\
\hline $\mathrm{H}$ & -2.05671 & -3.76532 & 4.07024 \\
\hline C & -1.89989 & -4.02407 & 1.94557 \\
\hline $\mathrm{H}$ & -2.61097 & -4.84077 & 1.91232 \\
\hline C & -1.30430 & -3.58279 & 0.77177 \\
\hline $\mathrm{H}$ & -1.55632 & -4.06480 & -0.1653 \\
\hline C & 1.88993 & -2.81735 & -0.9644 \\
\hline C & 2.63657 & -2.61132 & -2.12728 \\
\hline $\mathrm{H}$ & 2.33797 & -1.84429 & -2.8346 \\
\hline C & 3.75465 & -3.38742 & -2.39098 \\
\hline $\mathrm{H}$ & 4.32065 & -3.22150 & -3.2997 \\
\hline C & 4.14789 & -4.37297 & -1.49423 \\
\hline $\mathrm{H}$ & 5.02057 & -4.97981 & -1.7025 \\
\hline C & 3.42273 & -4.57137 & -0.32824 \\
\hline $\mathrm{H}$ & 3.72702 & -5.33373 & 0.37884 \\
\hline C & 2.30157 & -3.79709 & -0.0612 \\
\hline $\mathrm{H}$ & 1.73936 & -3.96794 & 0.8495 \\
\hline
\end{tabular}




$\begin{array}{llll}\mathrm{C} & 2.50688 & 2.89430 & -0.18811 \\ \mathrm{H} & 1.63470 & 3.56016 & -0.13608 \\ \mathrm{C} & 3.55933 & 3.47393 & 0.75301 \\ \mathrm{H} & 3.19349 & 3.47343 & 1.78343 \\ \mathrm{H} & 4.45578 & 2.84476 & 0.73901 \\ \mathrm{C} & 3.93946 & 4.88741 & 0.32726 \\ \mathrm{H} & 3.06653 & 5.54068 & 0.43983 \\ \mathrm{H} & 4.70958 & 5.28609 & 0.99255 \\ \mathrm{C} & 4.41175 & 4.92514 & -1.11861 \\ \mathrm{H} & 5.34477 & 4.35556 & -1.20651 \\ \mathrm{H} & 4.65001 & 5.94955 & -1.41507 \\ \mathrm{C} & 3.37349 & 4.32739 & -2.05730 \\ \mathrm{H} & 2.47316 & 4.95349 & -2.04923 \\ \mathrm{H} & 3.74097 & 4.33031 & -3.08659 \\ \mathrm{C} & 2.99744 & 2.91322 & -1.63583 \\ \mathrm{H} & 3.87130 & 2.26337 & -1.74231 \\ \mathrm{H} & 2.22927 & 2.50544 & -2.30102 \\ \mathrm{C} & 3.10941 & 0.10307 & 0.82289 \\ \mathrm{H} & 2.59306 & -0.86712 & 0.83522 \\ \mathrm{C} & 3.67652 & 0.30466 & 2.22662 \\ \mathrm{H} & 2.86347 & 0.35653 & 2.95499 \\ \mathrm{H} & 4.21034 & 1.25823 & 2.28556 \\ \mathrm{C} & 4.62919 & -0.83082 & 2.57889 \\ \mathrm{H} & 5.04092 & -0.68092 & 3.58012 \\ \mathrm{H} & 4.06275 & -1.77067 & 2.61587 \\ \mathrm{C} & 5.74659 & -0.95924 & 1.55418 \\ \mathrm{H} & 6.37027 & -0.05797 & 1.58745 \\ \mathrm{H} & 6.40289 & -1.79575 & 1.80704 \\ \mathrm{C} & 5.18583 & -1.13079 & 0.15041 \\ \mathrm{H} & 4.64491 & -2.08179 & 0.09350 \\ \mathrm{H} & 5.99293 & -1.19116 & -0.58461 \\ \mathrm{C} & 4.22891 & -0.00208 & -0.20896 \\ \mathrm{H} & 3.81507 & -0.15692 & -1.21054 \\ & 4.78302 & 0.94211 & -0.24532\end{array}$

\begin{tabular}{|c|c|c|c|}
\hline $\mathrm{H}$ & -0.20966 & 2.66898 & -2.30855 \\
\hline $\mathrm{Fe}$ & -0.32317 & 0.06468 & 0.13194 \\
\hline$P$ & 1.01508 & 1.82723 & -0.39814 \\
\hline$P$ & 1.03727 & -1.81767 & 0.30113 \\
\hline 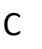 & -0.37086 & 0.33483 & 1.81378 \\
\hline 0 & -0.59508 & 0.50945 & 2.9476 \\
\hline C & -0.22047 & -2.97862 & 0.8209 \\
\hline $\mathrm{H}$ & -0.02776 & -4.00131 & 1.1158 \\
\hline C & -1.58065 & 2.56530 & -0.66571 \\
\hline $\mathrm{H}$ & -2.36980 & 3.01040 & -1.2852 \\
\hline 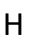 & -1.69845 & 3.01279 & 0.33766 \\
\hline C & 2.33672 & 1.61330 & -1.65715 \\
\hline$c$ & 3.68971 & 1.75244 & -1.34762 \\
\hline C & 1.97771 & 1.18388 & -2.93759 \\
\hline C & 4.66041 & 1.48497 & -2.30350 \\
\hline $\mathrm{H}$ & 3.99129 & 2.06986 & -0.35556 \\
\hline C & 2.94864 & 0.93027 & -3.89371 \\
\hline $\mathrm{H}$ & 0.93205 & 1.03673 & -3.18779 \\
\hline C & 4.29315 & 1.07865 & -3.57854 \\
\hline $\mathrm{H}$ & 5.70750 & 1.59826 & -2.04932 \\
\hline $\mathrm{H}$ & 2.65443 & 0.60593 & -4.88468 \\
\hline $\mathrm{H}$ & 5.05194 & 0.87337 & -4.3238 \\
\hline C & 1.79819 & 2.97051 & 0.81819 \\
\hline$C$ & 2.29294 & 4.19806 & 0.36665 \\
\hline C & 1.91717 & 2.65871 & 2.16945 \\
\hline$C$ & 2.89027 & 5.08555 & 1.24624 \\
\hline $\mathrm{H}$ & 2.21965 & 4.45859 & -0.68367 \\
\hline$C$ & 2.51765 & 3.54839 & 3.05161 \\
\hline $\mathrm{H}$ & 1.54741 & 1.71518 & 2.54611 \\
\hline$C$ & 3.00434 & 4.76199 & 2.59268 \\
\hline $\mathrm{H}$ & 3.26934 & 6.03184 & 0.87965 \\
\hline $\mathrm{H}$ & 2.60179 & 3.28701 & 4.09964 \\
\hline $\mathrm{H}$ & 3.47186 & 5.45618 & 3.28030 \\
\hline$C$ & -4.19120 & 1.26049 & -0.96452 \\
\hline$C$ & -5.29702 & 1.81296 & -0.32516 \\
\hline$C$ & -4.19535 & 1.18243 & -2.3573 \\
\hline$C$ & -6.38666 & 2.27137 & -1.05460 \\
\hline $\mathrm{H}$ & -5.30295 & 1.87670 & 0.7586 \\
\hline$C$ & -5.27838 & 1.64399 & -3.0895 \\
\hline $\mathrm{H}$ & -3.33449 & 0.76045 & -2.8659 \\
\hline$C$ & -6.38047 & 2.18723 & -2.4394 \\
\hline $\mathrm{H}$ & -7.24097 & 2.69537 & -0.5400 \\
\hline $\mathrm{H}$ & -5.26574 & 1.57935 & -4.1714 \\
\hline $\mathrm{H}$ & -7.22803 & 2.54560 & -3.0113 \\
\hline$C$ & -4.21013 & -1.37508 & 0.6308 \\
\hline$C$ & -5.36797 & -1.83770 & 0.0119 \\
\hline$C$ & -4.15887 & -1.35451 & 2.0248 \\
\hline$C$ & -6.45478 & -2.26508 & 0.7640 \\
\hline $\mathrm{H}$ & -5.41616 & -1.85577 & -1.0725 \\
\hline$C$ & -5.24029 & -1.78403 & 2.7784 \\
\hline $\mathrm{H}$ & -3.25771 & -1.00295 & 2.5172 \\
\hline$C$ & -6.39365 & -2.23889 & 2.1498 \\
\hline $\mathrm{H}$ & -7.34913 & -2.62098 & 0.2662 \\
\hline $\mathrm{H}$ & -5.18551 & -1.76414 & 3.8607 \\
\hline $\mathrm{H}$ & -7.23829 & -2.57489 & 2.7392 \\
\hline$C$ & 1.73521 & -2.56209 & -1.2915 \\
\hline$C$ & 3.10422 & -2.01399 & -1.6864 \\
\hline$C$ & 1.78043 & -4.08951 & -1.2540 \\
\hline
\end{tabular}

trans-(S,S)-4

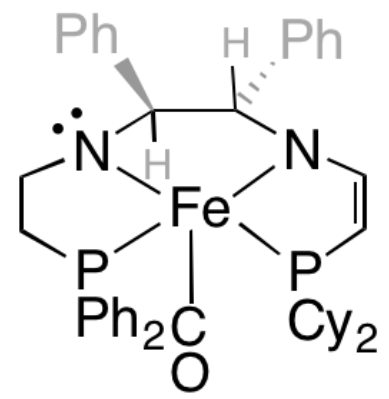

$H^{\circ}=-3799.394225$

$G^{\circ}=-3799.520870$

$\begin{array}{lll}H & -3.18392 & 1.02067\end{array}$

$\begin{array}{lll}\text { C } & -3.03885 & 0.70022\end{array}$

$\begin{array}{lll}\mathrm{H} & -3.23640 & -1.13284\end{array}$

$\begin{array}{lll}\text { C } & -3.06412 & -0.83743\end{array}$

$\begin{array}{lll}\mathrm{H} & -2.34423 & -3.20082\end{array}$

$\begin{array}{lll}\mathrm{N} & -1.71223 & 1.11679\end{array}$

$\begin{array}{lll}\mathrm{N} & -1.74022 & -1.26772\end{array}$

$\begin{array}{lll}\text { C } & -1.49176 & -2.52893\end{array}$

$\begin{array}{lll}\mathrm{H} & 0.02151 & 3.96712\end{array}$

$\begin{array}{lll}\text { C } & -0.22989 & 2.91193\end{array}$
0.88593

$-0.16335$

$-1.24012$

$-0.19136$

0.72134

$-0.61477$

0.21858

0.59582

$-1.13725$

$-1.24466$ 


$\begin{array}{llll}\text { H } & 1.00931 & -2.25917 & -2.05927 \\ \mathrm{C} & 3.55919 & -2.57587 & -3.02732 \\ \mathrm{H} & 3.83727 & -2.29935 & -0.92259 \\ \mathrm{H} & 3.09442 & -0.92367 & -1.71673 \\ \mathrm{C} & 2.26146 & -4.66839 & -2.57875 \\ \mathrm{H} & 2.45312 & -4.40813 & -0.44724 \\ \mathrm{H} & 0.79559 & -4.49057 & -1.01114 \\ \mathrm{C} & 3.60506 & -4.09485 & -2.99853 \\ \mathrm{H} & 4.53755 & -2.16463 & -3.29002 \\ \mathrm{H} & 2.86577 & -2.24541 & -3.80995 \\ \mathrm{H} & 2.31181 & -5.75820 & -2.51050 \\ \mathrm{H} & 1.51790 & -4.44445 & -3.35260 \\ \mathrm{H} & 3.90275 & -4.49022 & -3.97284 \\ \mathrm{H} & 4.37508 & -4.41671 & -2.28752 \\ \mathrm{C} & 2.46959 & -1.91519 & 1.49751 \\ \mathrm{C} & 3.32367 & -0.65059 & 1.40324 \\ \mathrm{C} & 1.99153 & -2.13124 & 2.93212 \\ \mathrm{H} & 3.07629 & -2.78105 & 1.19846 \\ \mathrm{C} & 4.47149 & -0.64188 & 2.40122 \\ \mathrm{H} & 2.67371 & 0.20810 & 1.60015 \\ \mathrm{H} & 3.70220 & -0.51035 & 0.38958 \\ \mathrm{C} & 3.15000 & -2.12904 & 3.92012 \\ \mathrm{H} & 1.28899 & -1.33363 & 3.20108 \\ \mathrm{H} & 1.43079 & -3.06499 & 3.00623 \\ \mathrm{C} & 3.96861 & -0.85132 & 3.82022 \\ \mathrm{H} & 5.01990 & 0.30150 & 2.32106 \\ \mathrm{H} & 5.18422 & -1.43490 & 2.14574 \\ \mathrm{H} & 2.77022 & -2.26447 & 4.93585 \\ \mathrm{H} & 3.79839 & -2.98958 & 3.71735 \\ \mathrm{H} & 4.80231 & -0.87245 & 4.52622 \\ \mathrm{H} & 3.34187 & 0.00213 & 4.10831\end{array}$

\begin{tabular}{|c|c|c|c|}
\hline C & -1.66890 & 0.64396 & -2.82496 \\
\hline H & -2.64704 & 0.75788 & -3.30550 \\
\hline $\mathrm{H}$ & -0.94681 & 0.40936 & -3.60704 \\
\hline C & -1.28754 & 1.92123 & -2.09808 \\
\hline $\mathrm{H}$ & -2.16592 & 2.38380 & -1.64878 \\
\hline $\mathrm{H}$ & -0.87751 & 2.64878 & -2.79684 \\
\hline C & -0.51437 & -1.54618 & 1.76619 \\
\hline $\mathrm{H}$ & -1.19846 & -1.85213 & 2.56324 \\
\hline c & 0.84168 & -1.59212 & 1.97581 \\
\hline $\mathrm{H}$ & 1.26005 & -1.86561 & 2.93648 \\
\hline C & 1.20411 & -0.64350 & -2.34276 \\
\hline C & -4.13292 & -0.54230 & -1.41776 \\
\hline C & -4.51672 & -1.69586 & -2.10094 \\
\hline $\mathrm{H}$ & -3.79804 & -2.49380 & -2.26035 \\
\hline C & -5.81348 & -1.83629 & -2.57252 \\
\hline $\mathrm{H}$ & -6.10005 & -2.73818 & -3.10004 \\
\hline C & -6.74451 & -0.82633 & -2.36588 \\
\hline H & -7.75755 & -0.93792 & -2.73294 \\
\hline C & -6.37150 & 0.32683 & -1.68956 \\
\hline $\mathrm{H}$ & -7.09232 & 1.11918 & -1.52753 \\
\hline C & -5.07266 & 0.46654 & -1.22076 \\
\hline $\mathrm{H}$ & -4.77853 & 1.36504 & -0.68605 \\
\hline C & -3.41182 & -1.24391 & 1.38813 \\
\hline C & -4.30476 & -2.27046 & 1.67678 \\
\hline $\mathrm{H}$ & -4.24849 & -3.19530 & 1.11174 \\
\hline C & -5.26310 & -2.11957 & 2.67148 \\
\hline $\mathrm{H}$ & -5.95143 & -2.92885 & 2.88475 \\
\hline C & -5.33715 & -0.93605 & 3.39133 \\
\hline $\mathrm{H}$ & -6.08349 & -0.81655 & 4.16747 \\
\hline C & -4.44590 & 0.09447 & 3.11431 \\
\hline $\mathrm{H}$ & -4.49794 & 1.02187 & 3.67336 \\
\hline C & -3.49190 & -0.05901 & 2.12044 \\
\hline $\mathrm{H}$ & -2.79503 & 0.74556 & 1.90537 \\
\hline C & 2.74973 & -2.84512 & 0.20067 \\
\hline $\mathrm{H}$ & 1.93924 & -3.57796 & 0.31929 \\
\hline C & -0.27268 & 1.79530 & 1.99688 \\
\hline $\mathrm{H}$ & 0.46909 & 1.00302 & 2.00318 \\
\hline C & -0.75185 & 2.29792 & 3.19750 \\
\hline $\mathrm{H}$ & -0.36879 & 1.90872 & 4.13379 \\
\hline C & -1.73167 & 3.28161 & 3.19683 \\
\hline $\mathrm{H}$ & -2.11578 & 3.66874 & 4.13294 \\
\hline C & -2.21872 & 3.76700 & 1.99083 \\
\hline $\mathrm{H}$ & -2.97850 & 4.53952 & 1.98307 \\
\hline C & -1.72910 & 3.27374 & 0.78834 \\
\hline $\mathrm{H}$ & -2.10848 & 3.68416 & -0.13981 \\
\hline C & -0.75482 & 2.27577 & 0.77895 \\
\hline C & 1.31841 & 2.74560 & -1.13796 \\
\hline C & 1.92162 & 2.69441 & -2.39733 \\
\hline $\mathrm{H}$ & 1.58135 & 1.97438 & -3.13328 \\
\hline C & 2.94505 & 3.57004 & -2.72628 \\
\hline $\mathrm{H}$ & 3.39820 & 3.51951 & -3.70909 \\
\hline C & 3.38271 & 4.51168 & -1.80357 \\
\hline $\mathrm{H}$ & 4.17999 & 5.19758 & -2.06269 \\
\hline C & 2.79410 & 4.56700 & -0.54889 \\
\hline $\mathrm{H}$ & 3.13042 & 5.29622 & 0.17864 \\
\hline C & 1.77292 & 3.68710 & -0.21447 \\
\hline $\mathrm{H}$ & 1.32516 & 3.74420 & 0.77083 \\
\hline$c$ & 3.80932 & -3.16201 & 1.2545 \\
\hline
\end{tabular}

$\Delta-(S, S)-\mathbf{3 A}$

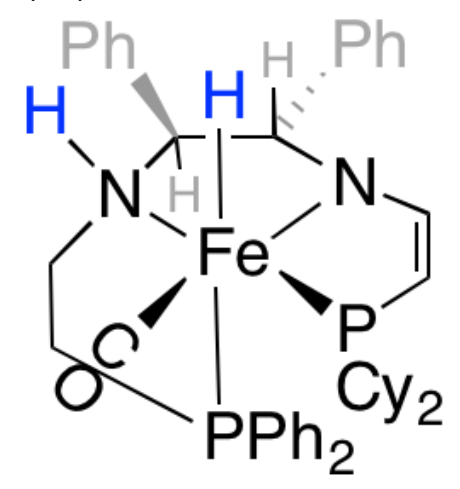

$H^{\circ}=-3800.586861$

$G^{\circ}=-3800.712638$

$\begin{array}{llll}\mathrm{Fe} & 0.17636 & -0.74125 & -0.94857\end{array}$

$\begin{array}{llll}\mathrm{H} & 0.08269 & -2.26980 & -1.22015\end{array}$

$\begin{array}{llll}\mathrm{P} & -0.03531 & 1.55343 & -0.75344\end{array}$

$\begin{array}{llll}P & 1.80523 & -1.25222 & 0.50950\end{array}$

$\begin{array}{llll}\mathrm{O} & 1.88811 & -0.64576 & -3.29297\end{array}$

$\begin{array}{llll}\mathrm{N} & -1.67287 & -0.50309 & -1.89067\end{array}$

$\begin{array}{llll}\mathrm{H} & -1.83676 & -1.34960 & -2.43010\end{array}$

$\begin{array}{llll}\mathrm{N} & -1.03398 & -1.10547 & 0.62066\end{array}$

$\begin{array}{llll}\text { C } & -2.74438 & -0.39515 & -0.86110\end{array}$

$\begin{array}{llll}\mathrm{H} & -2.65243 & 0.60327 & -0.42177\end{array}$

$\begin{array}{llll}\text { C } & -2.41138 & -1.38235 & 0.26464\end{array}$

$\begin{array}{llll}\text { H } & -2.50899 & -2.40096 & -0.15283\end{array}$ 


$\begin{array}{llll}\mathrm{H} & 3.38957 & -3.06221 & 2.25942 \\ \mathrm{H} & 4.62513 & -2.43454 & 1.18367 \\ \mathrm{C} & 4.38368 & -4.55900 & 1.05678 \\ \mathrm{H} & 5.15183 & -4.75795 & 1.80865 \\ \mathrm{H} & 3.59278 & -5.30039 & 1.21958 \\ \mathrm{C} & 4.94929 & -4.72540 & -0.34484 \\ \mathrm{H} & 5.80094 & -4.04617 & -0.47198 \\ \mathrm{H} & 5.34072 & -5.73636 & -0.48307 \\ \mathrm{C} & 3.89680 & -4.41335 & -1.39711 \\ \mathrm{H} & 3.09235 & -5.15543 & -1.33024 \\ \mathrm{H} & 4.31751 & -4.50835 & -2.40146 \\ \mathrm{C} & 3.30280 & -3.02189 & -1.21146 \\ \mathrm{H} & 2.50694 & -2.86119 & -1.94092 \\ \mathrm{H} & 4.07137 & -2.26968 & -1.41646 \\ \mathrm{C} & 3.04113 & 0.09698 & 0.90513 \\ \mathrm{H} & 2.41931 & 0.99720 & 0.79488 \\ \mathrm{C} & 3.58651 & 0.12652 & 2.33136 \\ \mathrm{H} & 2.76295 & 0.07039 & 3.04855 \\ \mathrm{H} & 4.21968 & -0.74637 & 2.51598 \\ \mathrm{C} & 4.39921 & 1.39489 & 2.55898 \\ \mathrm{H} & 3.73369 & 2.26378 & 2.47262 \\ \mathrm{H} & 4.79549 & 1.41198 & 3.57739 \\ \mathrm{C} & 5.52602 & 1.52319 & 1.54425 \\ \mathrm{H} & 6.24071 & 0.70542 & 1.69648 \\ \mathrm{H} & 6.08271 & 2.44963 & 1.70682 \\ \mathrm{C} & 4.99460 & 1.46819 & 0.11966 \\ \mathrm{H} & 5.81569 & 1.52134 & -0.60008 \\ \mathrm{H} & 4.36663 & 2.34667 & -0.06460 \\ \mathrm{C} & 4.16503 & 0.21430 & -0.12241 \\ \mathrm{H} & 3.75347 & 0.22395 & -1.13689 \\ \mathrm{H} & 4.81407 & -0.66581 & -0.05900\end{array}$

\begin{tabular}{|c|c|c|c|}
\hline $\mathrm{H}$ & -1.02224 & 0.20097 & -3.65921 \\
\hline C & -1.27250 & 1.81399 & -2.24743 \\
\hline$\Pi$ & -2.11299 & 2.32018 & -1.77286 \\
\hline $\mathrm{H}$ & -0.88119 & 2.49391 & -3.00271 \\
\hline C & -0.49476 & -1.48146 & 1.80045 \\
\hline $\mathrm{H}$ & -1.15417 & -1.76561 & 2.62645 \\
\hline C & 0.86944 & -1.51561 & 1.98052 \\
\hline H & 1.29934 & -1.75116 & 2.94648 \\
\hline C & 0.25282 & -2.49047 & -1.44880 \\
\hline C & -4.17795 & -0.49097 & -1.32045 \\
\hline C & -4.62403 & -1.65929 & -1.93817 \\
\hline $\mathrm{H}$ & -3.93937 & -2.48885 & -2.08674 \\
\hline C & -5.94005 & -1.77441 & -2.36077 \\
\hline $\mathrm{H}$ & -6.27457 & -2.68758 & -2.83828 \\
\hline C & -6.82930 & -0.72486 & -2.16816 \\
\hline $\mathrm{H}$ & -7.85747 & -0.81663 & -2.49655 \\
\hline C & -6.39527 & 0.44204 & -1.55491 \\
\hline $\mathrm{H}$ & -7.08361 & 1.26467 & -1.40305 \\
\hline C & -5.07697 & 0.55676 & -1.13642 \\
\hline $\mathrm{H}$ & -4.73541 & 1.46659 & -0.65159 \\
\hline C & -3.39643 & -1.07504 & 1.49611 \\
\hline C & -4.31328 & -2.04588 & 1.88494 \\
\hline $\mathrm{H}$ & -4.32007 & -3.00333 & 1.37409 \\
\hline C & -5.21539 & -1.79799 & 2.91291 \\
\hline $\mathrm{H}$ & -5.92323 & -2.56461 & 3.20524 \\
\hline C & -5.20787 & -0.57400 & 3.56586 \\
\hline $\mathrm{H}$ & -5.90887 & -0.38089 & 4.36902 \\
\hline C & -4.29323 & 0.40225 & 3.18640 \\
\hline $\mathrm{H}$ & -4.27870 & 1.36158 & 3.69134 \\
\hline C & -3.39777 & 0.15225 & 2.15924 \\
\hline $\mathrm{H}$ & -2.68359 & 0.91349 & 1.86264 \\
\hline C & 2.88962 & -2.65203 & 0.23550 \\
\hline $\mathrm{H}$ & 2.11583 & -3.43289 & 0.25228 \\
\hline C & -0.18976 & 1.92620 & 1.79152 \\
\hline $\mathrm{H}$ & 0.42350 & 1.03451 & 1.86816 \\
\hline C & -0.60385 & 2.57418 & 2.94695 \\
\hline $\mathrm{H}$ & -0.29090 & 2.19784 & 3.91429 \\
\hline C & -1.43213 & 3.68449 & 2.86183 \\
\hline $\mathrm{H}$ & -1.76536 & 4.18644 & 3.76223 \\
\hline C & -1.83403 & 4.15031 & 1.61655 \\
\hline $\mathrm{H}$ & -2.47514 & 5.02060 & 1.54304 \\
\hline C & -1.40715 & 3.51192 & 0.46018 \\
\hline $\mathrm{H}$ & -1.70953 & 3.90773 & -0.50266 \\
\hline C & -0.58322 & 2.38755 & 0.53548 \\
\hline C & 1.43475 & 2.54426 & -1.50229 \\
\hline C & 2.11356 & 2.19940 & -2.67273 \\
\hline $\mathrm{H}$ & 1.83180 & 1.29930 & -3.20894 \\
\hline C & 3.14504 & 2.99263 & -3.15078 \\
\hline $\mathrm{H}$ & 3.66046 & 2.71038 & -4.06102 \\
\hline C & 3.51579 & 4.14382 & -2.46765 \\
\hline $\mathrm{H}$ & 4.32142 & 4.76359 & -2.84214 \\
\hline C & 2.85210 & 4.49167 & -1.30076 \\
\hline $\mathrm{H}$ & 3.13850 & 5.38367 & -0.75646 \\
\hline C & 1.82103 & 3.69631 & -0.81693 \\
\hline $\mathrm{H}$ & 1.31767 & 3.98169 & 0.09913 \\
\hline C & 3.87433 & -2.98596 & 1.35356 \\
\hline $\mathrm{H}$ & 3.38157 & -2.93249 & 2.32812 \\
\hline$H$ & 4.68039 & -2.24392 & 1.36897 \\
\hline
\end{tabular}




$\begin{array}{llll}\mathrm{C} & 4.47335 & -4.37222 & 1.14322 \\ \mathrm{H} & 5.19795 & -4.59303 & 1.93130 \\ \mathrm{H} & 3.67753 & -5.12000 & 1.23868 \\ \mathrm{C} & 5.12134 & -4.50177 & -0.22748 \\ \mathrm{H} & 5.99061 & -3.83526 & -0.27806 \\ \mathrm{H} & 5.50483 & -5.51487 & -0.37246 \\ \mathrm{C} & 4.15024 & -4.13367 & -1.34045 \\ \mathrm{H} & 3.32886 & -4.85987 & -1.36390 \\ \mathrm{H} & 4.64429 & -4.19571 & -2.31349 \\ \mathrm{C} & 3.57544 & -2.73926 & -1.12808 \\ \mathrm{H} & 2.87685 & -2.48130 & -1.92800 \\ \mathrm{H} & 4.38837 & -2.01100 & -1.18603 \\ \mathrm{C} & 2.98039 & 0.29234 & 0.92402 \\ \mathrm{H} & 2.35235 & 1.17133 & 0.72448 \\ \mathrm{C} & 3.40402 & 0.39042 & 2.38782 \\ \mathrm{H} & 2.52453 & 0.34392 & 3.03581 \\ \mathrm{H} & 4.03297 & -0.46497 & 2.65675 \\ \mathrm{C} & 4.16931 & 1.68416 & 2.63461 \\ \mathrm{H} & 3.49256 & 2.53199 & 2.46530 \\ \mathrm{H} & 4.48140 & 1.74401 & 3.68042 \\ \mathrm{C} & 5.37003 & 1.81036 & 1.70841 \\ \mathrm{H} & 6.08971 & 1.01691 & 1.94318 \\ \mathrm{H} & 5.88964 & 2.75599 & 1.88242 \\ \mathrm{C} & 4.95147 & 1.69386 & 0.25067 \\ \mathrm{H} & 5.82232 & 1.75271 & -0.40758 \\ \mathrm{H} & 4.31069 & 2.54319 & -0.00929 \\ \mathrm{C} & 4.18663 & 0.40237 & -0.00530 \\ \mathrm{H} & 3.86856 & 0.34558 & -1.05116 \\ \mathrm{H} & 4.85716 & -0.44618 & 0.16460\end{array}$

\begin{tabular}{|c|c|c|c|}
\hline $\mathrm{H}$ & -2.62751 & -1.90146 & -1.01566 \\
\hline C & -0.77058 & -2.39408 & -1.94374 \\
\hline r & -0.21644 & -2.18923 & -2.86301 \\
\hline $\mathrm{H}$ & -0.96179 & -3.46802 & -1.91271 \\
\hline C & -0.64532 & 1.31320 & 1.72661 \\
\hline $\mathrm{H}$ & -1.32453 & 1.49474 & 2.56372 \\
\hline$C$ & 0.68582 & 1.64440 & 1.85190 \\
\hline $\mathrm{H}$ & 1.06580 & 2.12095 & 2.74688 \\
\hline C & 1.05760 & 0.36742 & -2.38972 \\
\hline C & -4.23781 & 0.25458 & -1.47317 \\
\hline C & -4.76497 & 0.90867 & -2.58686 \\
\hline $\mathrm{H}$ & -4.16881 & 1.66771 & -3.08350 \\
\hline C & -6.03292 & 0.60652 & -3.05980 \\
\hline $\mathrm{H}$ & -6.42486 & 1.12787 & -3.92496 \\
\hline C & -6.80078 & -0.35687 & -2.41975 \\
\hline $\mathrm{H}$ & -7.79380 & -0.59252 & -2.7829 \\
\hline$C$ & -6.29085 & -1.01310 & -1.30824 \\
\hline $\mathrm{H}$ & -6.88505 & -1.76439 & -0.80189 \\
\hline C & -5.01961 & -0.71168 & -0.83990 \\
\hline $\mathrm{H}$ & -4.63516 & -1.23592 & 0.02824 \\
\hline C & -3.48178 & 1.20345 & 1.33139 \\
\hline C & -3.64426 & 2.57798 & 1.15527 \\
\hline $\mathrm{H}$ & -3.08060 & 3.08960 & 0.38185 \\
\hline C & -4.50784 & 3.29700 & 1.96672 \\
\hline $\mathrm{H}$ & -4.62690 & 4.36336 & 1.81539 \\
\hline C & -5.21787 & 2.65462 & 2.97447 \\
\hline $\mathrm{H}$ & -5.89146 & 3.21783 & 3.60910 \\
\hline C & -5.05449 & 1.29072 & 3.16678 \\
\hline $\mathrm{H}$ & -5.59821 & 0.78369 & 3.95509 \\
\hline C & -4.19021 & 0.57302 & 2.34975 \\
\hline $\mathrm{H}$ & -4.05861 & -0.49428 & 2.50055 \\
\hline C & -0.50683 & -2.47910 & 0.95929 \\
\hline C & -0.17616 & -1.88818 & 2.17973 \\
\hline $\mathrm{H}$ & 0.54730 & -1.07775 & 2.20269 \\
\hline C & -0.77676 & -2.31252 & 3.35541 \\
\hline $\mathrm{H}$ & -0.51123 & -1.84305 & 4.29537 \\
\hline C & -1.72404 & -3.32759 & 3.32434 \\
\hline $\mathrm{H}$ & -2.20220 & -3.65333 & 4.24028 \\
\hline C & -2.05854 & -3.92325 & 2.11581 \\
\hline $\mathrm{H}$ & -2.79376 & -4.71866 & 2.08721 \\
\hline C & -1.45136 & -3.50529 & 0.93903 \\
\hline $\mathrm{H}$ & -1.72215 & -3.98572 & 0.00617 \\
\hline C & 1.80183 & -2.82938 & -0.74159 \\
\hline C & 2.51202 & -2.76637 & -1.94345 \\
\hline $\mathrm{H}$ & 2.18045 & -2.10835 & -2.73826 \\
\hline C & 3.63748 & -3.55233 & -2.13948 \\
\hline $\mathrm{H}$ & 4.17152 & -3.49697 & -3.08055 \\
\hline C & 4.07812 & -4.40569 & $-1.1362 C$ \\
\hline $\mathrm{H}$ & 4.95661 & -5.02003 & -1.29135 \\
\hline C & 3.38984 & -4.46234 & 0.06707 \\
\hline $\mathrm{H}$ & 3.72904 & -5.12073 & 0.85794 \\
\hline C & 2.26064 & -3.67881 & 0.26577 \\
\hline $\mathrm{H}$ & 1.73073 & -3.73927 & 1.20938 \\
\hline C & 2.33535 & 3.02183 & -0.11615 \\
\hline $\mathrm{H}$ & 1.43065 & 3.64129 & -0.03839 \\
\hline C & 3.36968 & 3.59545 & 0.85046 \\
\hline $\mathrm{H}$ & 3.00326 & 3.54270 & 1.87947 \\
\hline $\mathrm{H}$ & 4.28041 & 2.98815 & 0.8119 \\
\hline
\end{tabular}




$\begin{array}{llll}\mathrm{C} & 3.72778 & 5.03069 & 0.48689 \\ \mathrm{H} & 2.84139 & 5.66471 & 0.60520 \\ \mathrm{H} & 4.47794 & 5.41752 & 1.18148 \\ \mathrm{C} & 4.22571 & 5.12344 & -0.94681 \\ \mathrm{H} & 5.16341 & 4.56204 & -1.03825 \\ \mathrm{H} & 4.46211 & 6.15872 & -1.20485 \\ \mathrm{C} & 3.20215 & 4.55348 & -1.91627 \\ \mathrm{H} & 2.30136 & 5.17794 & -1.89372 \\ \mathrm{H} & 3.57810 & 4.59593 & -2.94173 \\ \mathrm{C} & 2.81908 & 3.12166 & -1.56144 \\ \mathrm{H} & 3.68232 & 2.46632 & -1.71698 \\ \mathrm{H} & 2.03680 & 2.77137 & -2.23715 \\ \mathrm{C} & 3.07957 & 0.23710 & 0.84676 \\ \mathrm{H} & 2.58067 & -0.74211 & 0.88378 \\ \mathrm{C} & 3.69232 & 0.45201 & 2.22958 \\ \mathrm{H} & 2.90387 & 0.49385 & 2.98553 \\ \mathrm{H} & 4.21562 & 1.41152 & 2.27075 \\ \mathrm{C} & 4.67497 & -0.66763 & 2.55070 \\ \mathrm{H} & 5.11890 & -0.50931 & 3.53685 \\ \mathrm{H} & 4.12716 & -1.61729 & 2.60833 \\ \mathrm{C} & 5.75825 & -0.77707 & 1.48775 \\ \mathrm{H} & 6.36528 & 0.13610 & 1.49851 \\ \mathrm{H} & 6.43924 & -1.60004 & 1.71932 \\ \mathrm{C} & 5.15341 & -0.96357 & 0.10437 \\ \mathrm{H} & 4.63005 & -1.92553 & 0.06931 \\ \mathrm{H} & 5.93638 & -1.01101 & -0.65726 \\ \mathrm{C} & 4.16042 & 0.14265 & -0.22660 \\ \mathrm{H} & 3.70917 & -0.03384 & -1.20870 \\ \mathrm{H} & 4.69273 & 1.09807 & -0.29701 \\ \mathrm{H} & -0.26371 & 1.90918 & -1.39031 \\ & & & \\ & & \end{array}$

$\begin{array}{llll}\mathrm{C} & -0.75568 & -2.35115 & -2.08352 \\ \mathrm{H} & -0.24633 & -2.09849 & -3.01543 \\ \mathrm{H} & -0.90946 & -3.43114 & -2.07710 \\ \mathrm{C} & -0.59630 & 1.29242 & 1.70925 \\ \mathrm{H} & -1.25372 & 1.47384 & 2.56434\end{array}$

$\begin{array}{llll}\text { C } & 0.74149 & 1.61655 & 1.80851\end{array}$

$\begin{array}{llll}\mathrm{H} & 1.13819 & 2.07063 & 2.70819\end{array}$

$\begin{array}{llll}\text { C } & -0.10590 & 2.08324 & -1.69515\end{array}$

$\begin{array}{llll}\text { C } & -4.26541 & 0.17767 & -1.38844\end{array}$

$\begin{array}{llll}\text { C } & -4.86328 & 0.84224 & -2.45896\end{array}$

H $\quad-4.31674 \quad 1.63545 \quad-2.95930$

$\begin{array}{llll}\text { C } & -6.13980 & 0.50728 & -2.88501\end{array}$

$\begin{array}{llll}\mathrm{H} & -6.58762 & 1.03771 & -3.71687\end{array}$

$\begin{array}{llll}\text { C } & -6.84456 & -0.50047 & -2.24079\end{array}$

H $\quad-7.84368 \quad-0.76181 \quad-2.56791$

C $\quad-6.26363 \quad-1.16782 \quad-1.17159$

H $\quad-6.80820 \quad-1.95366 \quad-0.66188$

$\begin{array}{llll}\text { C } & -4.98476 & -0.83302 & -0.74993\end{array}$

$\begin{array}{llll}\mathrm{H} & -4.54506 & -1.36697 & 0.08546\end{array}$

$\begin{array}{llll}\text { C } & -3.44895 & 1.09452 & 1.40206\end{array}$

$\begin{array}{llll}\text { C } & -3.67353 & 2.46375 & 1.25567\end{array}$

$\begin{array}{llll}\mathrm{H} & -3.16349 & 3.00993 & 0.46837\end{array}$

$\begin{array}{llll}\text { C } & -4.53078 & 3.13460 & 2.11382\end{array}$

$\begin{array}{llll}\mathrm{H} & -4.69925 & 4.19728 & 1.98537\end{array}$

$\begin{array}{llll}\text { C } & -5.17156 & 2.44881 & 3.13923\end{array}$

H $\quad-5.84041 \quad 2.97444 \quad 3.81006$

$\begin{array}{llll}\text { C } & -4.94563 & 1.08997 & 3.30217\end{array}$

$\begin{array}{llll}\mathrm{H} & -5.43537 & 0.54947 & 4.10353\end{array}$

$\begin{array}{llll}\text { C } & -4.08816 & 0.42059 & 2.43842\end{array}$

$\begin{array}{llll}\text { H } & -3.90717 & -0.64257 & 2.56565\end{array}$

$\begin{array}{llll}\text { C } & -0.36542 & -2.54281 & 0.79208\end{array}$

$\Lambda-(S, S)-3 \mathbf{B}$

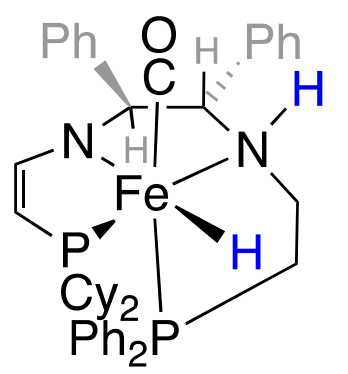

$H^{\circ}=-3800.576161$

$G^{\circ}=-3800.701765$

$\begin{array}{llll}\text { Fe } & 0.05919 & 0.49904 & -1.00587\end{array}$

$\begin{array}{llll}P & 0.34413 & -1.77073 & -0.71664\end{array}$

$\begin{array}{llll}P & 1.71008 & 1.24874 & 0.35635\end{array}$

$\begin{array}{llll}\mathrm{O} & -0.20583 & 3.12540 & -2.21375\end{array}$

$\begin{array}{llll}\mathrm{N} & -1.81017 & -0.14211 & -1.81107\end{array}$

$\begin{array}{llll}\mathrm{H} & -1.84726 & 0.25709 & -2.74444\end{array}$

$\begin{array}{llll}\mathrm{N} & -1.11042 & 0.76234 & 0.60887\end{array}$

$\begin{array}{llll}\text { C } & -2.85992 & 0.55616 & -0.99242\end{array}$

$\begin{array}{llll}\mathrm{H} & -2.71836 & 1.61626 & -1.21955\end{array}$

$\begin{array}{llll}\text { C } & -2.49926 & 0.36098 & 0.48698\end{array}$

$\begin{array}{llll}\mathrm{H} & -2.58949 & -0.71572 & 0.71938\end{array}$

$\begin{array}{llll}\text { C } & -2.05741 & -1.60459 & -1.94727\end{array}$

$\begin{array}{llll}\mathrm{H} & -2.72477 & -1.79883 & -2.79010\end{array}$

H $\quad-2.57653 \quad-1.94508 \quad-1.04956$

$\begin{array}{llll}\text { C } & -0.05858 & -1.95189 & 2.01900\end{array}$

$\begin{array}{llll}\text { H } & 0.59992 & -1.08896 & 2.04816\end{array}$

$\begin{array}{llll}\text { C } & -0.60772 & -2.43881 & 3.19571\end{array}$

$\begin{array}{llll}\mathrm{H} & -0.36113 & -1.96700 & 4.13977\end{array}$

$\begin{array}{llll}\text { C } & -1.48196 & -3.51700 & 3.16029\end{array}$

$\begin{array}{llll}\text { H } & -1.92110 & -3.89177 & 4.07702\end{array}$

$\begin{array}{llll}\text { C } & -1.79401 & -4.11262 & 1.94567\end{array}$

$\mathrm{H} \quad-2.47245 \quad-4.95681 \quad 1.91275$

$\begin{array}{llll}\text { C } & -1.23646 & -3.63245 & 0.76833\end{array}$

H $\quad-1.48642 \quad-4.11648 \quad-0.16844$

$\begin{array}{llll}\text { C } & 1.87534 & -2.73938 & -1.03445\end{array}$

$\begin{array}{llll}\text { C } & 2.62388 & -2.46222 & -2.17992\end{array}$

$\begin{array}{llll}\mathrm{H} & 2.32725 & -1.64587 & -2.83023\end{array}$

$\begin{array}{llll}\mathrm{C} & 2.32725 & -1.64587 & -2.83023 \\ \mathrm{H} & 3.74044 & -3.22336 & -2.49115\end{array}$

$\begin{array}{llll}\mathrm{H} & 4.30945 & -2.99967 & -3.38569\end{array}$

$\begin{array}{llll}\text { C } & 4.12940 & -4.26612 & -1.66001\end{array}$

$\begin{array}{llll}\mathrm{H} & 5.00193 & -4.85977 & -1.90433\end{array}$

$\begin{array}{llll}\text { C } & 3.39920 & -4.53943 & -0.51257\end{array}$

$\begin{array}{llll}\mathrm{H} & 3.69885 & -5.34736 & 0.14417\end{array}$

$\begin{array}{llll}\text { C } & 2.27935 & -3.78077 & -0.19879\end{array}$

$\begin{array}{llll}\mathrm{H} & 2.71513 & -4.00906 & 0.69790\end{array}$

$\begin{array}{llll}\text { C } & 2.51653 & 2.86578 & -0.13439\end{array}$

$\begin{array}{llll}\mathrm{H} & 1.63470 & 3.52198 & -0.16602\end{array}$

$\begin{array}{llll}\text { C } & 3.47566 & 3.46073 & 0.89398\end{array}$

$\begin{array}{llll}\mathrm{H} & 3.02782 & 3.44711 & 1.89137\end{array}$

$\begin{array}{llll}\mathrm{H} & 4.38138 & 2.84641 & 0.94893\end{array}$

$\begin{array}{llll}\text { C } & 3.86252 & 4.88389 & 0.50855\end{array}$ 


$\begin{array}{llll}\mathrm{H} & 2.97072 & 5.51953 & 0.55468 \\ \mathrm{H} & 4.56854 & 5.29243 & 1.23621 \\ \mathrm{C} & 4.44999 & 4.94240 & -0.89382 \\ \mathrm{H} & 5.40257 & 4.39918 & -0.90594 \\ \mathrm{H} & 4.68338 & 5.97475 & -1.16609 \\ \mathrm{C} & 3.51159 & 4.31874 & -1.91728 \\ \mathrm{H} & 2.59649 & 4.91927 & -1.98716 \\ \mathrm{H} & 3.96625 & 4.33461 & -2.91108 \\ \mathrm{C} & 3.14365 & 2.89268 & -1.52854 \\ \mathrm{H} & 4.04826 & 2.27850 & -1.54306 \\ \mathrm{H} & 2.46551 & 2.45311 & -2.26404 \\ \mathrm{C} & 3.07791 & 0.06890 & 0.86989 \\ \mathrm{H} & 2.56749 & -0.90351 & 0.83341 \\ \mathrm{C} & 3.58677 & 0.22944 & 2.30107 \\ \mathrm{H} & 2.74486 & 0.26569 & 2.99689 \\ \mathrm{H} & 4.12043 & 1.17950 & 2.40754 \\ \mathrm{C} & 4.51888 & -0.92032 & 2.66243 \\ \mathrm{H} & 4.89176 & -0.79813 & 3.68267 \\ \mathrm{H} & 3.94491 & -1.85643 & 2.65460 \\ \mathrm{C} & 5.67617 & -1.03455 & 1.68072 \\ \mathrm{H} & 6.30830 & -0.14256 & 1.76536 \\ \mathrm{H} & 6.31199 & -1.88577 & 1.93721 \\ \mathrm{C} & 5.17362 & -1.15796 & 0.25005 \\ \mathrm{H} & 4.62742 & -2.10139 & 0.13959 \\ \mathrm{H} & 6.01100 & -1.20366 & -0.45148 \\ \mathrm{C} & 4.24354 & -0.00800 & -0.11172 \\ \mathrm{H} & 3.87622 & -0.11784 & -1.13681 \\ \mathrm{H} & 4.80706 & 0.93089 & -0.08109 \\ \mathrm{H} & 0.92115 & 0.13196 & -2.26175\end{array}$

$\begin{array}{llll}\mathrm{C} & 0.57219 & -0.14193 & 1.59248 \\ \mathrm{O} & 0.87368 & -0.18699 & 2.71859 \\ \mathrm{H} & -0.14022 & 0.29621 & -1.66189 \\ \mathrm{C} & 0.16165 & 3.07960 & 0.48725 \\ \mathrm{H} & -0.03120 & 4.11118 & 0.75242 \\ \mathrm{C} & 1.58119 & -2.55822 & -0.71233 \\ \mathrm{H} & 2.37103 & -3.12299 & -1.21837 \\ \mathrm{H} & 1.63458 & -2.79557 & 0.35480 \\ \mathrm{H} & 1.78076 & -0.89574 & -1.85436 \\ \mathrm{C} & -2.48732 & -1.83251 & -1.53625 \\ \mathrm{C} & -3.78104 & -1.96568 & -1.03041 \\ \mathrm{C} & -2.32172 & -1.57025 & -2.89734 \\ \mathrm{C} & -4.88195 & -1.84417 & -1.86707 \\ \mathrm{H} & -3.93398 & -2.15992 & 0.02543 \\ \mathrm{C} & -3.42215 & -1.46226 & -3.73392 \\ \mathrm{H} & -1.32744 & -1.43311 & -3.30763 \\ \mathrm{C} & -4.70568 & -1.59452 & -3.22060 \\ \mathrm{H} & -5.87987 & -1.94573 & -1.45761 \\ \mathrm{H} & -3.27618 & -1.26320 & -4.78888\end{array}$

$\begin{array}{llll}\mathrm{H} & -5.56487 & -1.49942 & -3.87338\end{array}$

$\begin{array}{llll}\text { C } & -1.57537 & -2.96978 & 0.91955\end{array}$

$\begin{array}{llll}\text { C } & -2.09158 & -4.22053 & 0.56474\end{array}$

$\begin{array}{llll}\text { C } & -1.46314 & -2.65781 & 2.27067\end{array}$

$\begin{array}{llll}\text { C } & -2.48898 & -5.12540 & 1.53485\end{array}$

H $\quad-2.19004 \quad-4.48735 \quad-0.48222$

$\begin{array}{llll}\text { C } & -1.86336 & -3.56402 & 3.24578\end{array}$

H $\quad-1.06396 \quad-1.70124 \quad 2.57711$

$\begin{array}{llll}\text { C } & -2.37818 & -4.79743 & 2.88080\end{array}$

$\begin{array}{llll}\text { H } & -2.88764 & -6.08870 & 1.24008\end{array}$

$\begin{array}{llll}H & -1.77045 & -3.29915 & 4.29229\end{array}$

trans-(S,S)-3'

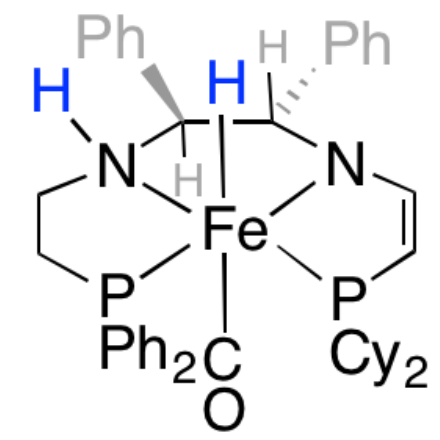

$\begin{array}{lccc}H^{\circ}=-3800.577825 & & \\ G^{\circ}= & -3800.705558 & & \\ H & 2.98942 & -0.74939 & 0.79090 \\ \mathrm{C} & 3.04293 & -0.57849 & -0.28918 \\ \mathrm{H} & 3.18970 & 1.07729 & -1.62303 \\ \mathrm{C} & 3.01779 & 0.94552 & -0.53895 \\ \mathrm{H} & 2.26740 & 3.36432 & 0.23953 \\ \mathrm{~N} & 1.78511 & -1.10802 & -0.85771 \\ \mathrm{~N} & 1.69591 & 1.40713 & -0.17920 \\ \mathrm{C} & 1.43609 & 2.65659 & 0.18118 \\ \mathrm{H} & -0.01818 & -3.94540 & -1.18125 \\ \mathrm{C} & 0.22216 & -2.88773 & -1.28628 \\ \mathrm{H} & 0.20535 & -2.64806 & -2.35206 \\ \mathrm{Fe} & 0.21474 & 0.04127 & -0.12693 \\ \mathrm{P} & -1.02733 & -1.81038 & -0.41776 \\ \mathrm{P} & -1.11318 & 1.86671 & 0.18990\end{array}$

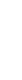

(n)

(n)

(

然

che

(a)

西

$\mathrm{H}$
$\mathrm{H}$

$-2.69185$

$-5.50369$

3.63994

$\begin{array}{ll}4.29256 & -1.22298\end{array}$

$5.26964-1.68963$

$\begin{array}{ll}4.52279 & -1.31067\end{array}$

$\begin{array}{ll}6.45536 & -2.22646\end{array}$

$\begin{array}{ll}5.09746-1.62260 \\ 5.70463 & -1.85171\end{array}$

$5.70463-1.85171$

$3.77373-0.95073$

$\begin{array}{ll}6.67587 & -2.30772\end{array}$

$\begin{array}{ll}7.20724 & -2.58280\end{array}$

$\begin{array}{ll}5.87071 & -1.91393\end{array}$

$\begin{array}{ll}7.60001 & -2.72715\end{array}$

$\begin{array}{ll}4.16763 & 1.59843\end{array}$

$\begin{array}{ll}5.29211 & 2.03551\end{array}$

$\begin{array}{ll}4.15317 & 1.70217\end{array}$

$\begin{array}{ll}6.38527 & 2.55906\end{array}$

$\begin{array}{ll}5.31083 & 1.95673\end{array}$

$\begin{array}{ll}5.24135 & 2.22627\end{array}$

$\begin{array}{ll}3.27678 & 1.37051\end{array}$

6.36301

7.25449

5.21724

7.21337

$\begin{array}{ll}\text { C } & -2.09547 \\ \text { C } & -3.37697 \\ \text { C } & -2.39133\end{array}$

C $\quad-2.39133$

H -1.40520

C -4.04828

2.65406

2.89362

2.30043

3.06374

2.53032

1.75758

4.02520

$-0.82697$

0.04807

$-2.19975$

$-0.43380$

1.11735

$-2.68372$

$-2.89836$

$-1.80159$

0.26008

$-3.75257$

$-2.18034$

0.19134

$-0.50160$

1.58226

0.17721

$-1.58389$

2.26209

2.13034

1.56096

$-0.37666$

3.34292

2.09268

$-1.26992$

$-1.56456$

$-1.16833$

$2.37798-2.11006$

2.26301

$-2.83534$ 


$\begin{array}{llll}\mathrm{H} & -4.07529 & 1.87990 & -0.72725 \\ \mathrm{H} & -3.16699 & 0.68902 & -1.64327 \\ \mathrm{C} & -3.07633 & 4.53929 & -2.42916 \\ \mathrm{H} & -3.03836 & 4.21406 & -0.30251 \\ \mathrm{H} & -1.46827 & 4.58176 & -0.99323 \\ \mathrm{C} & -4.33833 & 3.75340 & -2.74861 \\ \mathrm{H} & -4.96860 & 1.70312 & -3.02224 \\ \mathrm{H} & -3.39018 & 2.06813 & -3.69084 \\ \mathrm{H} & -3.30301 & 5.60355 & -2.32438 \\ \mathrm{H} & -2.37768 & 4.45635 & -3.26982 \\ \mathrm{H} & -4.78768 & 4.11281 & -3.67773 \\ \mathrm{H} & -5.08097 & 3.92790 & -1.96106 \\ \mathrm{C} & -2.37947 & 1.92987 & 1.57211 \\ \mathrm{C} & -3.08733 & 0.58000 & 1.68059 \\ \mathrm{C} & -1.77731 & 2.32717 & 2.91653 \\ \mathrm{H} & -3.11906 & 2.69037 & 1.28716 \\ \mathrm{C} & -4.12368 & 0.55034 & 2.79293 \\ \mathrm{H} & -2.32766 & -0.18167 & 1.88009 \\ \mathrm{H} & -3.53927 & 0.30318 & 0.72486 \\ \mathrm{C} & -2.81915 & 2.29785 & 4.02784 \\ \mathrm{H} & -0.96303 & 1.63734 & 3.16594 \\ \mathrm{H} & -1.32262 & 3.31785 & 2.84860 \\ \mathrm{C} & -3.50305 & 0.94254 & 4.12481 \\ \mathrm{H} & -4.57298 & -0.44545 & 2.85512 \\ \mathrm{H} & -4.94017 & 1.24186 & 2.55296 \\ \mathrm{H} & -2.35433 & 2.56178 & 4.98117 \\ \mathrm{H} & -3.57498 & 3.06773 & 3.83281 \\ \mathrm{H} & -4.25805 & 0.95012 & 4.91486 \\ \mathrm{H} & -2.76324 & 0.18467 & 4.41296\end{array}$

\begin{tabular}{|c|c|c|c|}
\hline & 96 & 346 & -2 \\
\hline & .478686 & .167024 & 190205 \\
\hline & 0.094893 & -3.127266 & 06982 \\
\hline & .110450 & -4.145111 & 0.517047 \\
\hline & 1.604027 & 2.548099 & -0.610248 \\
\hline & 2.392049 & 3.167216 & -1.050189 \\
\hline & -1.674632 & 2.645832 & 0.477302 \\
\hline & -1.914523 & 1.059754 & -1.951015 \\
\hline & 2.552004 & 2.068090 & -1.232121 \\
\hline & 3.776958 & 2.002685 & -0.5653 \\
\hline & 2.549881 & 2.171969 & -2.623922 \\
\hline & 4.969103 & 2.046857 & -1.272285 \\
\hline & 3.800279 & 1.912574 & 0.515371 \\
\hline & 3.743918 & 2.220371 & -3.329117 \\
\hline & 1.613600 & 2.208609 & -3.168329 \\
\hline & 4.956272 & 2.154900 & -2.656269 \\
\hline & 5.910945 & 1.994220 & -0.739181 \\
\hline & 3.725077 & 2.307074 & -4.408906 \\
\hline & 5.887443 & 2.187367 & -3.208735 \\
\hline & 1.303354 & 2.708179 & 1.265970 \\
\hline & 1.927376 & 3.958602 & 1.217566 \\
\hline & 0.871444 & 2.224456 & 2.496654 \\
\hline & 2.122623 & 4.696354 & 2.373661 \\
\hline & 2.265397 & 4.358923 & 0.267850 \\
\hline & 1.064417 & 2.965781 & 3.656318 \\
\hline r & 0.376735 & 1.260960 & 2.542667 \\
\hline & & & \\
\hline F & 2.609794 & 5.662485 & 2.318534 \\
\hline P & 0.720845 & 2.573968 & 4.606303 \\
\hline P & 1.843984 & 4.777377 & 4.502147 \\
\hline C & -4.302823 & 1.188395 & -0.719369 \\
\hline ( & & & \\
\hline & -4.659452 & 1.326155 & -2.060829 \\
\hline 0 & -6.413384 & 2.185327 & -0.092122 \\
\hline h & -4.921631 & 1.52 & 1.30 \\
\hline C & -5.877532 & 1.888046 & -2.413704 \\
\hline H & & & \\
\hline & -6.759613 & 2.3 & -1.4 \\
\hline H & -7.0 & & 0.68 \\
\hline $\mathrm{H}$ & -6.141604 & 1.988401 & -3.459732 \\
\hline H & -7.712150 & 2.751677 & -1.705945 \\
\hline C & -4.108908 & & 0.0 \\
\hline C & -5.239756 & -2.112234 & -0.625010 \\
\hline C & -4.060956 & -1.843701 & 1.446015 \\
\hline C & -6.306981 & -2.686350 & 0.054373 \\
\hline $\mathrm{H}$ & -5.283357 & -1.989352 & -1.70242 \\
\hline C & -5.123057 & -2.418154 & 2.12626 \\
\hline $\mathrm{H}$ & -3.178419 & -1.517552 & 1.98838 \\
\hline C & -6.251809 & -2.838790 & 1.43199 \\
\hline $\mathrm{H}$ & -7.181397 & -3.015851 & -0.49434 \\
\hline $\mathrm{H}$ & -5.073145 & -2.537964 & 3.20214 \\
\hline $\mathrm{H}$ & -7.081710 & -3.287935 & 1.96420 \\
\hline C & 2.441639 & -2.456511 & -1.01197 \\
\hline C & 3.447246 & -1.426028 & -1.51877 \\
\hline & 3.168062 & -3.716864 & -0.54886 \\
\hline $\mathrm{H}$ & 1.811526 & -2.738912 & -1.86730 \\
\hline C & 4.276872 & -2.002514 & -2.65873 \\
\hline & 4.119547 & -1.129991 & -0.706 \\
\hline
\end{tabular}

trans-(S,S)-3

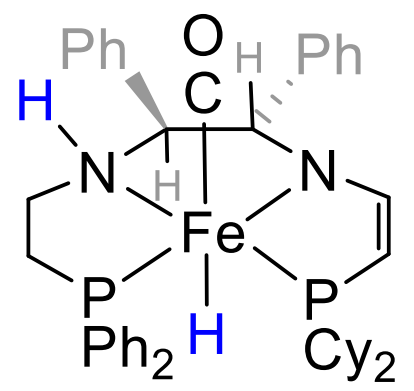

\begin{tabular}{|c|c|c|c|}
\hline \multicolumn{4}{|c|}{$H^{\circ}=-3800.581199$} \\
\hline \multicolumn{4}{|c|}{$G^{\circ}=-3800.708676$} \\
\hline $\mathrm{H}$ & -2.870744 & 0.622606 & 0.758750 \\
\hline C & -3.011212 & 0.525155 & -0.321986 \\
\hline $\mathrm{H}$ & -3.183626 & -1.053441 & -1.750198 \\
\hline C & -2.985439 & -0.978294 & -0.664181 \\
\hline $\mathrm{H}$ & -2.200568 & -3.425443 & -0.029352 \\
\hline $\mathrm{N}$ & -1.805150 & 1.122984 & -0.939626 \\
\hline $\mathrm{N}$ & -1.655036 & -1.442770 & -0.350389 \\
\hline $\mathrm{C}$ & -1.377555 & -2.706475 & -0.062957 \\
\hline H & -0.016468 & 4.004453 & -0.872701 \\
\hline c & -0.237096 & 2.962167 & -1.105501 \\
\hline $\mathrm{H}$ & -0.183428 & 2.839009 & -2.190016 \\
\hline $\mathrm{Fe}$ & -0.169652 & -0.101475 & -0.480903 \\
\hline$P$ & 0.983218 & 1.801377 & -0.314933 \\
\hline 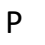 & 1.135093 & -1.834085 & 0.184349 \\
\hline & 0.276025 & -0.397483 & -2.16635 \\
\hline
\end{tabular}




$\begin{array}{llll}\text { H } & 2.937919 & -0.515906 & -1.841001 \\ \text { C } & 4.005203 & -4.303508 & -1.679365 \\ \text { H } & 3.827452 & -3.468108 & 0.291266 \\ \text { H } & 2.456494 & -4.460215 & -0.178407 \\ \text { C } & 4.985543 & -3.281153 & -2.235589 \\ \text { H } & 5.000698 & -1.262121 & -3.009887 \\ \text { H } & 3.615893 & -2.214705 & -3.508157 \\ \text { H } & 4.537153 & -5.193162 & -1.332225 \\ \text { H } & 3.336104 & -4.637503 & -2.480760 \\ \text { H } & 5.544105 & -3.705587 & -3.073592 \\ \text { H } & 5.726448 & -3.039259 & -1.464226 \\ \text { C } & 1.971100 & -1.887528 & 1.859505 \\ \text { C } & 3.020529 & -0.791548 & 2.020545 \\ \text { C } & 0.951551 & -1.834758 & 2.993783 \\ \text { H } & 2.469427 & -2.864994 & 1.905618 \\ \text { C } & 3.679059 & -0.821211 & 3.393779 \\ \text { H } & 2.533856 & 0.180710 & 1.872305 \\ \text { H } & 3.782910 & -0.872393 & 1.242870 \\ \text { C } & 1.627391 & -1.891066 & 4.356411 \\ \text { H } & 0.373462 & -0.906142 & 2.914214 \\ \text { H } & 0.231031 & -2.649437 & 2.885649 \\ \text { C } & 2.649489 & -0.776338 & 4.511707 \\ \text { H } & 4.383202 & 0.010534 & 3.485841 \\ \text { H } & 4.273214 & -1.738185 & 3.486985 \\ \text { H } & 0.876470 & -1.836382 & 5.148880 \\ \text { H } & 2.127589 & -2.859879 & 4.473145 \\ \text { H } & 3.140289 & -0.836014 & 5.486367 \\ \text { H } & 2.131732 & 0.189810 & 4.485002\end{array}$

$\Delta-c i s-\beta-(S, S)-5 A$

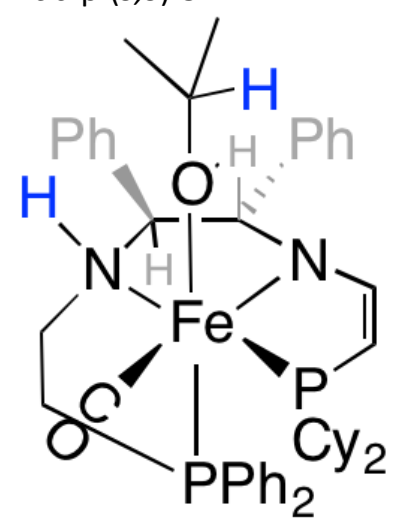

$\begin{array}{lccc}H^{\circ}=-3993.712445 & & \\ G^{\circ}=-3993.849797 & & \\ \mathrm{Fe} & -0.08136 & 0.63881 & -0.77735 \\ \mathrm{P} & -0.02176 & -1.65890 & -0.99062 \\ \mathrm{P} & -1.79372 & 0.88320 & 0.74649 \\ \mathrm{O} & -1.71491 & 1.13454 & -3.13851 \\ \mathrm{~N} & 1.75907 & 0.42190 & -1.68257 \\ \mathrm{H} & 1.85800 & 1.38725 & -2.00275 \\ \mathrm{~N} & 1.07323 & 0.61996 & 0.86726 \\ \mathrm{C} & 2.81421 & 0.12719 & -0.67898 \\ \mathrm{H} & 2.72848 & -0.93731 & -0.43606 \\ \mathrm{C} & 2.47309 & 0.89814 & 0.60318 \\ \mathrm{H} & 2.61373 & 1.96907 & 0.38703 \\ \mathrm{C} & 1.74803 & -0.51977 & -2.81240 \\ \mathrm{H} & 2.73955 & -0.60110 & -3.27175 \\ \mathrm{H} & 1.07166 & -0.10974 & -3.56289\end{array}$

\begin{tabular}{|c|c|c|c|}
\hline & 047 & -1.87651 & \\
\hline & 2.09941 & -2.45352 & \\
\hline & 0.85789 & -2.46056 & 3.153 \\
\hline & 0.53380 & 0.91909 & 1.048 \\
\hline & 20608 & 1.07743 & 89 \\
\hline & -0.82293 & 0.99703 & 2.241 \\
\hline & -1.24489 & 1.12342 & חבר 20 \\
\hline & -1.07942 & 0.86871 & -2.199 \\
\hline & 4.20524 & 0.39927 & -1.183 \\
\hline & 4.55846 & 1.66717 & -1.645 \\
\hline & 3.81325 & 2.45728 & -1.674 \\
\hline & 5.85520 & 1.92884 & -2.060 \\
\hline & 6.11848 & 2.91858 & -2.414 \\
\hline & 6.81707 & 0.92720 & -2.018 \\
\hline & 7.83045 & 1.13377 & -2.341 \\
\hline & 6.47438 & -0.33842 & -1.5643 \\
\hline & 7.21954 & -1.12429 & -1.532 \\
\hline & 5.17514 & -0.59 & -1.150 \\
\hline & 4.90548 & $-1.5 \varepsilon$ & -0.789 \\
\hline & 3.42352 & 0.51294 & 1.7122 \\
\hline & 4.34716 & 1.42742 & 2.208 \\
\hline & 4.35432 & 2.43861 & 1.813 \\
\hline & 5.25461 & 1.05696 & 3.193 \\
\hline & 5.96788 & 1.78090 & 3.5693 \\
\hline & 5.24521 & -0.23574 & 3.6968 \\
\hline & 5.95047 & -0.5 & 4.466 \\
\hline & 4.32312 & -1.15 & 3.211 \\
\hline & 4.30830 & -2.16 & 3.6002 \\
\hline & 3.42174 & -0.78364 & 2.2268 \\
\hline & 2.70064 & -1.50120 & 1.848 \\
\hline & -2.85091 & 2.43121 & 0.766 \\
\hline & -2.07422 & 3.19577 & 0.884 \\
\hline & 0.12830 & -2.37 & 1.692 \\
\hline & -0.51212 & -1.5 & $4 \angle Z$ \\
\hline & 0.50878 & -3.1 & 2.783 \\
\hline & 0.14 & -2 & 72 \\
\hline & 1.36 & -4 & 2.608 \\
\hline & 1.66863 & -4.82307 & 3.459 \\
\hline & 1.82570 & -4.53897 & 1.337 \\
\hline & 2.48683 & -5.38486 & 1.192 \\
\hline & 1.43457 & -3.77915 & 0.243 \\
\hline & 1.78530 & -4.06025 & 0.742 \\
\hline & 0.58651 & -2.68402 & .411 \\
\hline & -1.40772 & -2.69337 & 1.637 \\
\hline & -1.99432 & -2.3 & 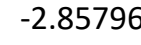 \\
\hline & -1.64050 & -1.4 & \\
\hline & -3.01615 & -3.1 & -3.392 \\
\hline & -3.45716 & -2.83829 & -4.341 \\
\hline & -3.46442 & -4.24855 & -2.720 \\
\hline & -4.25896 & -4.85220 & -3.141 \\
\hline & -2.88845 & -4.59904 & -1.50 \\
\hline & -3.23214 & -5.47772 & $-0.97 €$ \\
\hline & -1.87184 & -3.82421 & -0.965 \\
\hline & -1.43684 & -4.11256 & -0.016 \\
\hline & -3.77052 & 2.56631 & 1.980 \\
\hline & -3.23 & 2.31153 & - \\
\hline & -4 & 1.86205 & \\
\hline & & 3.98 & $2.0 t$ \\
\hline
\end{tabular}




\begin{tabular}{llll}
$\mathrm{H}$ & -4.98402 & 4.08319 & 2.92722 \\
$\mathrm{H}$ & -3.48099 & 4.67497 & 2.24688 \\
$\mathrm{C}$ & -5.03392 & 4.39201 & 0.78799 \\
$\mathrm{H}$ & -5.93614 & 3.77926 & 0.67382 \\
$\mathrm{H}$ & -5.37525 & 5.42801 & 0.85420 \\
$\mathrm{C}$ & -4.14751 & 4.19961 & -0.43404 \\
$\mathrm{H}$ & -3.29893 & 4.89413 & -0.38452 \\
$\mathrm{H}$ & -4.69618 & 4.44865 & -1.34604 \\
$\mathrm{C}$ & -3.61804 & 2.77286 & -0.51143 \\
$\mathrm{H}$ & -2.98619 & 2.64872 & -1.39277 \\
$\mathrm{H}$ & -4.46223 & 2.09199 & -0.64103 \\
$\mathrm{C}$ & -2.96594 & -0.57700 & 0.90395 \\
$\mathrm{H}$ & -2.35417 & -1.41900 & 0.55559 \\
$\mathrm{C}$ & -3.39202 & -0.92081 & 2.32989 \\
$\mathrm{H}$ & -2.51309 & -0.99827 & 2.97605 \\
$\mathrm{H}$ & -4.01053 & -0.11797 & 2.74386 \\
$\mathrm{C}$ & -4.17628 & -2.22719 & 2.34596 \\
$\mathrm{H}$ & -3.51354 & -3.04184 & 2.02553 \\
$\mathrm{H}$ & -4.48697 & -2.46754 & 3.36590 \\
$\mathrm{C}$ & -5.38160 & -2.16617 & 1.41926 \\
$\mathrm{H}$ & -6.08680 & -1.41628 & 1.79734 \\
$\mathrm{H}$ & -5.91672 & -3.11901 & 1.42517 \\
$\mathrm{C}$ & -4.96886 & -1.79866 & 0.00213 \\
$\mathrm{H}$ & -5.84596 & -1.71442 & -0.64482 \\
$\mathrm{H}$ & -4.35351 & -2.60203 & -0.41529 \\
$\mathrm{C}$ & -4.17429 & -0.50026 & -0.02517 \\
$\mathrm{H}$ & -3.85905 & -0.26607 & -1.04781 \\
$\mathrm{H}$ & -4.82644 & 0.31736 & 0.29704 \\
$\mathrm{O}$ & 0.57024 & 2.56288 & -1.03157 \\
$\mathrm{H}$ & -0.12756 & 3.76253 & -0.93066 \\
$\mathrm{H}$ & -1.21435 & 3.59388 & -0.98391 \\
$\mathrm{H}$ & -0.01967 & 3.78224 & 1.23361 \\
$\mathrm{C}$ & 0.17664 & 4.45467 & 0.39434 \\
$\mathrm{H}$ & -0.41558 & 5.36317 & 0.54107 \\
$\mathrm{H}$ & -0.23377 & 4.73410 & 0.43983 \\
$\mathrm{H}$ & 1.30830 & 4.8583616 & -2.11877 \\
$\mathrm{H}$ & -2369 & 4.18777 & -3.05085 \\
\hline
\end{tabular}

\begin{tabular}{|c|c|c|c|}
\hline $\mathrm{N}$ & -1.78587 & -0.14312 & -1.62851 \\
\hline $\mathrm{H}$ & -1.83413 & 0.42779 & -2.46843 \\
\hline N & -1.06479 & 0.28325 & 0.89617 \\
\hline $\mathrm{C}$ & -2.83972 & 0.37050 & -0.69561 \\
\hline $\mathrm{H}$ & -2.69931 & 1.45238 & -0.73060 \\
\hline C & -2.46463 & -0.07516 & 0.72189 \\
\hline $\mathrm{H}$ & -2.56775 & -1.17319 & 0.78677 \\
\hline C & -1.99124 & -1.56316 & -2.02172 \\
\hline $\mathrm{H}$ & -2.62115 & -1.62400 & -2.91200 \\
\hline $\mathrm{H}$ & -2.53697 & -2.06006 & -1.21782 \\
\hline C & -0.67012 & -2.26211 & -2.23201 \\
\hline $\mathrm{H}$ & -0.14781 & -1.86617 & -3.10637 \\
\hline $\mathrm{H}$ & -0.80167 & -3.33323 & -2.39252 \\
\hline C & -0.56882 & 0.68862 & 2.05576 \\
\hline $\mathrm{H}$ & -1.22765 & 0.70903 & 2.92811 \\
\hline C & 0.74540 & 1.06969 & 2.20884 \\
\hline $\mathrm{H}$ & 1.12712 & 1.38380 & 3.17151 \\
\hline C & 1.06312 & 0.56868 & -2.18554 \\
\hline C & -4.23657 & 0.04336 & -1.15881 \\
\hline C & -4.82740 & 0.86291 & -2.12139 \\
\hline $\mathrm{H}$ & -4.28069 & 1.72864 & -2.48257 \\
\hline C & -6.09335 & 0.58921 & -2.61584 \\
\hline $\mathrm{H}$ & -6.53459 & 1.24057 & -3.36083 \\
\hline C & -6.79678 & -0.51358 & -2.14981 \\
\hline $\mathrm{H}$ & -7.78823 & -0.72865 & -2.52958 \\
\hline C & -6.22389 & -1.33607 & -1.18999 \\
\hline $\mathrm{H}$ & -6.76718 & -2.19706 & -0.81917 \\
\hline C & -4.95405 & -1.06176 & -0.70048 \\
\hline $\mathrm{H}$ & -4.51966 & -1.71715 & 0.04674 \\
\hline C & -3.40159 & 0.52473 & 1.74266 \\
\hline C & -3.59110 & 1.90592 & 1.81123 \\
\hline $\mathrm{H}$ & -3.04895 & 2.55374 & \\
\hline C & -4.44723 & 2.45539 & 2.75228 \\
\hline $\mathrm{H}$ & -4.58690 & 3.52928 & 2.79142 \\
\hline C & -5.12343 & 1.63345 & 3.64672 \\
\hline $\mathrm{H}$ & -5.79180 & 2.06408 & 4.38266 \\
\hline C & -4.93334 & 0.26057 & 3.59541 \\
\hline $\mathrm{H}$ & -5.45099 & -0.38641 & 4.29376 \\
\hline C & -4.07617 & -0.28726 & 2.64911 \\
\hline $\mathrm{H}$ & -3.92472 & -1.36169 & 2.60937 \\
\hline C & -0.33773 & -2.82584 & 0.61434 \\
\hline C & 0.03001 & -2.47556 & 1.91401 \\
\hline $\mathrm{H}$ & 0.73697 & -1.66678 & 2.07258 \\
\hline C & -0.51717 & -3.13522 & 3.00350 \\
\hline $\mathrm{H}$ & -0.22513 & -2.84947 & 4.00718 \\
\hline C & -1.44416 & -4.15099 & 2.80780 \\
\hline $\mathrm{H}$ & -1.88033 & -4.66029 & 3.65871 \\
\hline C & -1.81119 & -4.51128 & 1.51911 \\
\hline $\mathrm{H}$ & -2.52891 & -5.30712 & 1.36026 \\
\hline C & -1.25818 & -3.85625 & 0.42630 \\
\hline $\mathrm{H}$ & -1.55236 & -4.15751 & -0.57184 \\
\hline C & 1.93135 & -2.81143 & -1.16181 \\
\hline C & 2.62813 & -2.51416 & -2.33557 \\
\hline $\mathrm{H}$ & 2.28674 & -1.71853 & -2.98658 \\
\hline C & 3.75354 & -3.24212 & -2.69070 \\
\hline $\mathrm{H}$ & 4.27897 & -3.00190 & -3.60717 \\
\hline C & 4.20202 & -4.27633 & -1.87999 \\
\hline $\mathrm{H}$ & 5.07903 & -4.84678 & -2.1603 \\
\hline
\end{tabular}




\begin{tabular}{|c|c|c|c|}
\hline C & 3.52283 & -4.57247 & -0.70732 \\
\hline $\mathrm{H}$ & 3.86724 & -5.37503 & -0.06616 \\
\hline C & 2.39719 & -3.84393 & -0.34655 \\
\hline $\mathrm{H}$ & 1.87619 & -4.09135 & 0.57066 \\
\hline$C$ & 2.35308 & 2.77796 & 0.47520 \\
\hline $\mathrm{H}$ & 1.39440 & 3.30419 & 0.36507 \\
\hline C & 3.07572 & 3.36761 & 1.68471 \\
\hline 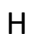 & 2.52338 & 3.16532 & 2.60573 \\
\hline H & 4.05616 & 2.88856 & 1.79209 \\
\hline $\mathrm{C}$ & 3.26815 & 4.86970 & 1.50745 \\
\hline $\mathrm{H}$ & 2.28361 & 5.35223 & 1.49233 \\
\hline $\mathrm{H}$ & 3.80064 & 5.28585 & 2.36669 \\
\hline C & 4.00699 & 5.19386 & 0.21710 \\
\hline $\mathrm{H}$ & 5.03507 & 4.81926 & 0.28866 \\
\hline $\mathrm{H}$ & 4.08499 & 6.27581 & 0.08444 \\
\hline C & 3.33178 & 4.55535 & -0.98811 \\
\hline $\mathrm{H}$ & 2.34537 & 5.01024 & -1.14032 \\
\hline $\mathrm{H}$ & 3.90630 & 4.75432 & -1.89659 \\
\hline C & 3.16448 & 3.05369 & -0.79056 \\
\hline $\mathrm{H}$ & 4.15685 & 2.60354 & -0.70484 \\
\hline $\mathrm{H}$ & 2.69598 & 2.60280 & -1.66669 \\
\hline C & 3.13948 & -0.12872 & 0.99418 \\
\hline $\mathrm{H}$ & 2.64762 & -1.10649 & 0.88753 \\
\hline$c$ & 3.74630 & -0.10333 & 2.39581 \\
\hline $\mathrm{H}$ & 2.95819 & -0.16293 & 3.15050 \\
\hline $\mathrm{H}$ & 4.26653 & 0.84626 & 2.56066 \\
\hline C & 4.72823 & -1.25623 & 2.56721 \\
\hline $\mathrm{H}$ & 5.16975 & -1.22976 & 3.56665 \\
\hline $\mathrm{H}$ & & -2.20332 & 2.49868 \\
\hline C & 5.81474 & -1.23130 & 1.50198 \\
\hline $\mathrm{H}$ & 6.43209 & -0.33577 & 1.63978 \\
\hline $\mathrm{H}$ & 6.48524 & -2.08611 & 1.62033 \\
\hline C & 5.21482 & -1.21629 & 0.10390 \\
\hline $\mathrm{H}$ & 4.68415 & -2.15804 & -0.07092 \\
\hline $\mathrm{H}$ & 6.00108 & -1.16016 & -0.65395 \\
\hline C & 4.23685 & -0.06193 & -0.06378 \\
\hline $\mathrm{H}$ & 3.80676 & -0.06231 & -1.07090 \\
\hline $\mathrm{H}$ & 4.78278 & 0.88049 & 0.04369 \\
\hline 0 & -0.59946 & 2.28355 & -0.76540 \\
\hline C & -0.34265 & 3.19966 & -1.78255 \\
\hline $\mathrm{H}$ & 0.73709 & 3.23999 & -2.03005 \\
\hline C & -1.08345 & 2.87009 & -3.07544 \\
\hline $\mathrm{H}$ & -0.90760 & 3.61631 & -3.85494 \\
\hline $\mathrm{H}$ & -0.76946 & 1.90518 & -3.48047 \\
\hline $\mathrm{H}$ & -2.16193 & 2.82754 & -2.89174 \\
\hline C & -0.74225 & 4.58238 & -1.28533 \\
\hline $\mathrm{H}$ & -0.21230 & 4.82813 & -0.3622 \\
\hline $\mathrm{H}$ & -0.52745 & 5.36413 & -2.01821 \\
\hline $\mathrm{H}$ & -1.81417 & 4.61002 & -1.066 \\
\hline
\end{tabular}

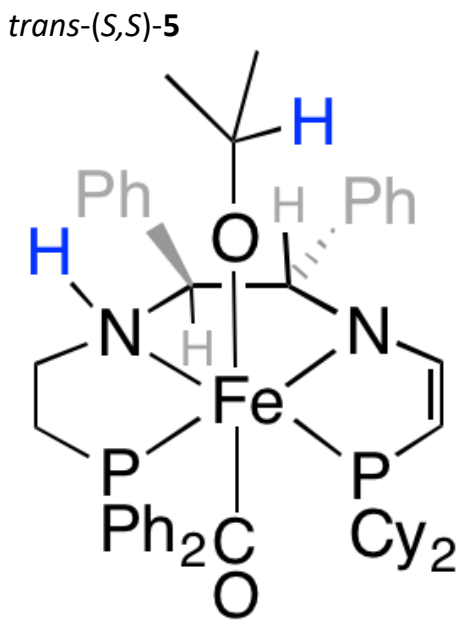

\begin{tabular}{|c|c|c|c|}
\hline \multicolumn{4}{|c|}{$H^{\circ}=-3993.715729$} \\
\hline \multicolumn{4}{|c|}{$G^{\circ}=-3993.853788$} \\
\hline $\mathrm{Fe}$ & -0.21949 & -0.03893 & -0.07657 \\
\hline$P$ & 1.01753 & 1.91626 & -0.33589 \\
\hline $\mathrm{P}$ & 1.16380 & -1.81303 & 0.44575 \\
\hline $\mathrm{H}$ & -3.00281 & 0.80279 & 0.89385 \\
\hline C & -3.05007 & 0.56703 & -0.17447 \\
\hline $\mathrm{H}$ & -3.31456 & -1.17547 & -1.37950 \\
\hline 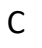 & -3.02501 & -0.97273 & -0.33558 \\
\hline $\mathrm{H}$ & -2.19558 & -3.37221 & 0.37473 \\
\hline $\mathrm{N}$ & -1.80093 & 1.08246 & -0.76971 \\
\hline 政 & -1.66212 & -1.41705 & -0.10065 \\
\hline C & -1.37655 & -2.65131 & 0.30740 \\
\hline त & -0.05070 & 3.92803 & 074 \\
\hline C & -0.25515 & 2.85742 & -1.31134 \\
\hline $\mathrm{H}$ & -0.21609 & 2.51182 & -2.34621 \\
\hline c & -0.58904 & 0.17764 & 1.60601 \\
\hline 0 & -0.93021 & 0.26144 & 2.71853 \\
\hline C & -0.10493 & -3.0 & 486 \\
\hline $\mathrm{H}$ & 0.08710 & -4.07553 & 0.94087 \\
\hline C & -1.61413 & 2.54014 & -0.72148 \\
\hline $\mathrm{H}$ & -2.40732 & 3.05933 & -1.26921 \\
\hline H & -1.68043 & 2.85309 & 0.32565 \\
\hline 11 & -1.71979 & 0.78072 & -1.74457 \\
\hline C & 2.55683 & 1.96212 & -1.33050 \\
\hline C & 3.79197 & 2.05315 & -0.68150 \\
\hline c & 2.53740 & 1.76135 & -2.71146 \\
\hline$C$ & 4.97530 & 1.94014 & -1.39423 \\
\hline 1 & 3.83027 & 2.21112 & 0.39081 \\
\hline 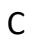 & 3.72468 & 1.64783 & -3.42195 \\
\hline $\mathrm{H}$ & 1.59355 & 1.67173 & -3.23188 \\
\hline$C$ & 4.94501 & 1.73228 & -2.76718 \\
\hline $\mathrm{H}$ & 5.92332 & 2.01287 & -0.87467 \\
\hline $\mathrm{H}$ & 3.69198 & 1.48754 & -4.49343 \\
\hline $\mathrm{H}$ & 5.86974 & 1.63775 & -3.32365 \\
\hline C & 1.39844 & 3.15978 & 0.97959 \\
\hline C & 1.94958 & 4.38772 & 0.59674 \\
\hline$C$ & 1.12369 & 2.94787 & 2.32739 \\
\hline C & 2.22931 & 5.36444 & 1.53737 \\
\hline $\mathrm{H}$ & 2.16798 & 4.57840 & -0.44859 \\
\hline$C$ & 1.40488 & 3.92791 & 3.27246 \\
\hline 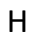 & 0.68722 & 2.01682 & 2.65787 \\
\hline$C$ & 1.96069 & 5.13497 & 2.88141 \\
\hline
\end{tabular}




\begin{tabular}{|c|c|c|c|}
\hline $\mathrm{H}$ & 2.65862 & 6.30738 & 080 \\
\hline $\mathrm{H}$ & 1.18627 & 3.74021 & 4.31689 \\
\hline $\mathrm{H}$ & 2.18238 & 5.89748 & 3.61814 \\
\hline C & -4.30987 & 1.16527 & -0.74349 \\
\hline C & -5.31653 & 1.61689 & 0.10503 \\
\hline C & -4.51596 & 1.22065 & -2.12156 \\
\hline C & -6.50812 & 2.10956 & -0.40914 \\
\hline $\mathrm{H}$ & -5.16354 & 1.57290 & 1.17852 \\
\hline C & -5.70323 & 1.71772 & -2.63771 \\
\hline $\mathrm{H}$ & -3.73958 & 0.87172 & -2.79633 \\
\hline C & -6.70402 & 2.16060 & -1.78199 \\
\hline $\mathrm{H}$ & -7.28383 & 2.45492 & 0.26376 \\
\hline $\mathrm{H}$ & -5.85013 & 1.75689 & -3.71043 \\
\hline $\mathrm{H}$ & -7.63244 & 2.54635 & -2.18529 \\
\hline C & -4.08813 & -1.59099 & 0.54324 \\
\hline C & -5.28344 & -2.03891 & -0.01109 \\
\hline C & -3.92218 & -1.66145 & 1.92640 \\
\hline C & -6.29718 & -2.54026 & 0.79531 \\
\hline $\mathrm{H}$ & -5.41999 & -1.98658 & -1.08674 \\
\hline C & -4.93107 & -2.16365 & 2.73347 \\
\hline $\mathrm{H}$ & -2.99101 & -1.32322 & 2.36865 \\
\hline C & -6.12377 & -2.60249 & 2.17018 \\
\hline $\mathrm{H}$ & -7.22215 & -2.88441 & 0.34796 \\
\hline $\mathrm{H}$ & -4.78799 & -2.21278 & 3.80649 \\
\hline $\mathrm{H}$ & -6.91122 & -2.99578 & 2.80180 \\
\hline$c$ & 2.36783 & -2.51372 & -0.80608 \\
\hline C & 3.58436 & -1.62013 & -1.03051 \\
\hline C & 2.78382 & -3.94884 & -0.48346 \\
\hline $\mathrm{H}$ & 1.79044 & -2.54339 & -1.73416 \\
\hline C & 4.52319 & -2.19407 & -2.08479 \\
\hline $\mathrm{H}$ & 4.13927 & -1.51019 & -0.09044 \\
\hline $\mathrm{H}$ & 3.25600 & -0.61773 & -1.31974 \\
\hline C & 3.71198 & -4.50491 & -1.55649 \\
\hline $\mathrm{H}$ & 3.29799 & -3.98249 & 0.48451 \\
\hline $\mathrm{H}$ & 1.89979 & -4.58317 & -0.38818 \\
\hline C & 4.93260 & -3.61973 & -1.75138 \\
\hline $\mathrm{H}$ & 5.40361 & -1.55404 & -2.18637 \\
\hline $\mathrm{H}$ & 4.02167 & -2.18204 & -3.05973 \\
\hline $\mathrm{H}$ & 4.01153 & -5.52404 & -1.29875 \\
\hline $\mathrm{H}$ & 3.16230 & -4.57552 & -2.50299 \\
\hline $\mathrm{H}$ & 5.57940 & -4.02517 & -2.53337 \\
\hline $\mathrm{H}$ & 5.52746 & -3.61898 & -0.83020 \\
\hline C & 2.23971 & -1.72616 & 1.98329 \\
\hline C & 2.76633 & -0.30430 & 2.16857 \\
\hline C & 1.54160 & -2.22447 & 3.24497 \\
\hline $\mathrm{H}$ & 3.09890 & -2.38163 & 1.79272 \\
\hline C & 3.65427 & -0.16935 & 3.39483 \\
\hline $\mathrm{H}$ & 1.91221 & 0.36864 & 2.27656 \\
\hline $\mathrm{H}$ & 3.29106 & 0.02706 & 1.26802 \\
\hline C & 2.43120 & -2.07165 & 4.47273 \\
\hline $\mathrm{H}$ & 0.61260 & -1.66518 & 3.39748 \\
\hline $\mathrm{H}$ & 1.24608 & -3.26878 & 3.12022 \\
\hline C & 2.92335 & -0.64199 & 4.64180 \\
\hline $\mathrm{H}$ & 3.97718 & 0.87007 & 3.50572 \\
\hline $\mathrm{H}$ & 4.56576 & -0.76338 & 3.25798 \\
\hline $\mathrm{H}$ & 1.89268 & -2.40073 & 5.36503 \\
\hline $\mathrm{H}$ & 3.29680 & -2.73747 & 4.37417 \\
\hline $\mathrm{H}$ & 3.56664 & -0.56048 & 5.52127 \\
\hline
\end{tabular}

$\begin{array}{llll}\mathrm{H} & 2.06535 & 0.01786 & 4.82488 \\ \mathrm{H} & -2.26528 & -0.97290 & -2.96368 \\ \mathrm{C} & -1.42639 & -1.01720 & -3.65818 \\ \mathrm{H} & -1.40480 & -0.07648 & -4.21801 \\ \mathrm{H} & -1.63160 & -1.82329 & -4.36793 \\ \mathrm{H} & -0.17310 & -2.17157 & -2.37175 \\ \mathrm{C} & -0.10329 & -1.21359 & -2.92445 \\ \mathrm{C} & 1.01768 & -1.33863 & -3.95265 \\ \mathrm{H} & 0.90474 & -2.22929 & -4.57658 \\ \mathrm{H} & 1.02300 & -0.46803 & -4.61545 \\ \mathrm{O} & 0.16904 & -0.15522 & -2.05915 \\ \mathrm{H} & 1.99461 & -1.38367 & -3.46859\end{array}$

$\Delta-(S, S)-6 \mathbf{A}$ (from trans-(S,S)-C1)

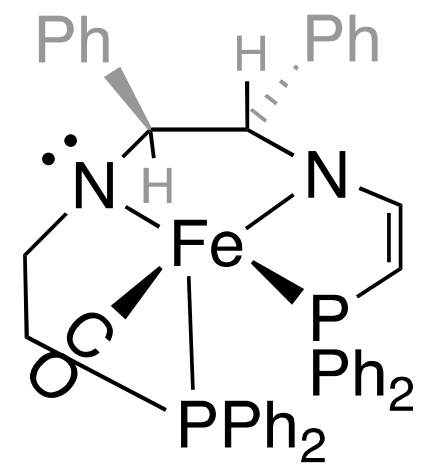

\begin{tabular}{lccc}
\multicolumn{4}{l}{$H^{\circ}=-3792.279027$} \\
$G^{\circ}=-3792.399730$ & & \\
$\mathrm{Fe}$ & 0.21202 & -0.77386 & -0.77230 \\
$\mathrm{P}$ & 0.24284 & 1.40358 & -0.96447 \\
$\mathrm{P}$ & 1.82448 & -1.27493 & 0.74476 \\
$\mathrm{O}$ & 2.07268 & -1.21597 & -2.97224 \\
$\mathrm{~N}$ & -1.46416 & -0.63716 & -1.73333 \\
$\mathrm{~N}$ & -0.96776 & -1.07432 & 0.74381 \\
$\mathrm{C}$ & -2.56340 & -0.30459 & -0.82530 \\
$\mathrm{H}$ & -2.49215 & 0.74160 & -0.46893 \\
$\mathrm{C}$ & -2.38723 & -1.18127 & 0.41945 \\
$\mathrm{H}$ & -2.61580 & -2.21732 & 0.12064 \\
$\mathrm{C}$ & -1.39249 & 0.30573 & -2.84765 \\
$\mathrm{H}$ & -2.36034 & 0.39696 & -3.35886 \\
$\mathrm{H}$ & -0.68711 & -0.08984 & -3.58265 \\
$\mathrm{C}$ & -0.93115 & 1.68800 & -2.38543 \\
$\mathrm{H}$ & -1.77057 & 2.28551 & -2.02835 \\
$\mathrm{H}$ & -0.44751 & 2.26646 & -3.17352 \\
$\mathrm{C}$ & -0.49673 & -1.40602 & 1.95652 \\
$\mathrm{H}$ & -1.21848 & -1.63227 & 2.74425 \\
$\mathrm{C}$ & 0.84605 & -1.45424 & 2.22451 \\
$\mathrm{H}$ & 1.23655 & -1.75405 & 3.18812 \\
$\mathrm{C}$ & 1.31648 & -0.97438 & -2.11874 \\
$\mathrm{C}$ & -3.93332 & -0.46092 & -1.43908 \\
$\mathrm{C}$ & -4.29451 & -1.63605 & -2.09699 \\
$\mathrm{H}$ & -3.56104 & -2.42800 & -2.20384 \\
$\mathrm{C}$ & -5.57125 & -1.79260 & -2.61535 \\
$\mathrm{H}$ & -5.83855 & -2.71207 & -3.12314 \\
$\mathrm{C}$ & -6.50837 & -0.77445 & -2.48520 \\
$\mathrm{H}$ & -7.50557 & -0.89713 & -2.89066 \\
$\mathrm{C}$ & -6.15804 & 0.40215 & -1.83789 \\
$\mathrm{H}$ & -6.88157 & 1.20257 & -1.73668
\end{tabular}




\begin{tabular}{|c|c|c|c|}
\hline C & -4.87812 & 0.55524 & -1.32078 \\
\hline $\mathrm{H}$ & -4.60239 & 1.47438 & -0.81066 \\
\hline 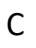 & -3.34857 & -0.79143 & 1.51358 \\
\hline C & -4.42951 & -1.60977 & 1.82916 \\
\hline $\mathrm{H}$ & -4.54242 & -2.55967 & 1.31644 \\
\hline C & -5.36195 & -1.22041 & 2.78256 \\
\hline $\mathrm{H}$ & -6.19865 & -1.86869 & 3.01493 \\
\hline C & -5.22162 & -0.00516 & 3.43716 \\
\hline $\mathrm{H}$ & -5.94628 & 0.29928 & 4.18280 \\
\hline C & -4.14336 & 0.81804 & 3.13359 \\
\hline $\mathrm{H}$ & -4.02480 & 1.76885 & 3.64092 \\
\hline C & -3.21745 & 0.42769 & 2.17848 \\
\hline $\mathrm{H}$ & -2.37738 & 1.07118 & 1.93995 \\
\hline C & 0.11639 & 2.01034 & 1.73377 \\
\hline $\mathrm{H}$ & 0.84489 & 1.21284 & 1.81910 \\
\hline C & -0.30691 & 2.67818 & 2.87202 \\
\hline $\mathrm{H}$ & 0.10598 & 2.40839 & 3.83716 \\
\hline C & -1.26940 & 3.67415 & 2.77215 \\
\hline $\mathrm{H}$ & -1.61214 & 4.19161 & 3.65998 \\
\hline C & -1.79504 & 4.00202 & 1.52990 \\
\hline $\mathrm{H}$ & -2.54707 & 4.77726 & 1.44602 \\
\hline C & -1.36004 & 3.34373 & 0.38686 \\
\hline $\mathrm{H}$ & -1.77543 & 3.62644 & -0.57256 \\
\hline C & -0.39970 & 2.33733 & 0.47988 \\
\hline C & 1.78606 & 2.29393 & -1.40324 \\
\hline C & 2.32788 & 2.10634 & -2.67738 \\
\hline $\mathrm{H}$ & 1.81662 & 1.48073 & -3.40049 \\
\hline C & 3.51541 & 2.72518 & -3.03414 \\
\hline $\mathrm{H}$ & 3.92087 & 2.57502 & -4.02735 \\
\hline C & 4.17853 & 3.53867 & -2.12441 \\
\hline $\mathrm{H}$ & 5.10624 & 4.02270 & -2.40427 \\
\hline C & 3.64704 & 3.73011 & -0.85767 \\
\hline $\mathrm{H}$ & 4.15849 & 4.36334 & -0.14260 \\
\hline C & 2.45961 & 3.10998 & -0.49489 \\
\hline $\mathrm{H}$ & 2.05832 & 3.27039 & 0.49877 \\
\hline C & 3.25110 & -0.16805 & 1.05712 \\
\hline C & 3.39519 & 0.53311 & 2.25200 \\
\hline $\mathrm{H}$ & 2.65859 & 0.41014 & 3.03933 \\
\hline C & 4.47929 & 1.38151 & 2.44154 \\
\hline $\mathrm{H}$ & 4.58328 & 1.92013 & 3.37595 \\
\hline C & 5.43043 & 1.52986 & 1.44307 \\
\hline $\mathrm{H}$ & 6.27658 & 2.18976 & 1.59225 \\
\hline C & 5.29503 & 0.83162 & 0.24839 \\
\hline $\mathrm{H}$ & 6.03346 & 0.94786 & -0.53608 \\
\hline C & 4.21037 & -0.00753 & 0.05324 \\
\hline $\mathrm{H}$ & 4.10880 & -0.54827 & -0.88266 \\
\hline C & 2.65474 & -2.90441 & 0.48590 \\
\hline C & 3.82404 & -3.26292 & 1.15688 \\
\hline $\mathrm{H}$ & 4.31419 & -2.55052 & 1.81196 \\
\hline C & 4.36690 & -4.52898 & 0.99226 \\
\hline $\mathrm{H}$ & 5.27672 & -4.79590 & 1.51684 \\
\hline C & 3.74833 & -5.45158 & 0.15875 \\
\hline $\mathrm{H}$ & 4.17641 & -6.43820 & 0.02890 \\
\hline C & 2.58201 & -5.10499 & -0.50972 \\
\hline $\mathrm{H}$ & 2.09730 & -5.81947 & -1.16431 \\
\hline C & 2.04057 & -3.83804 & -0.34957 \\
\hline $\mathrm{H}$ & 1.13193 & -3.56414 & -0.87866 \\
\hline
\end{tabular}

$\Delta-(S, S)-6 \mathbf{B}$ (from trans-(S,S)-C1)

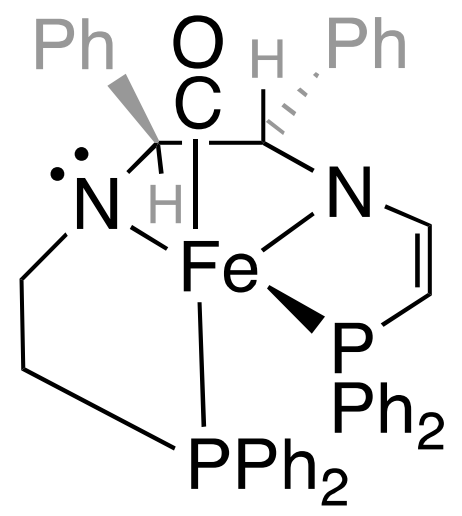

$H^{\circ}=-3792.250009$

$G^{\circ}=-3792.372174$

Fe $\quad-0.24488 \quad 0.87738 \quad-0.93605$

P $\quad-0.28214 \quad-1.55341 \quad-0.88268$

$\begin{array}{llll}P & -1.97819 & 1.32295 & 0.51997\end{array}$

O $\quad 0.22440 \quad 3.69240 \quad-1.48932$

N $\quad \begin{array}{llll}\text { N } & .48152 & 0.49638 & -1.89423\end{array}$

$\begin{array}{llll}\mathrm{N} & 0.88475 & 0.96015 & 0.63103\end{array}$

$\begin{array}{llll}\text { C } & 2.50558 & 0.18811 & -0.90967\end{array}$

$\begin{array}{llll}\text { H } & 2.39112 & -0.82696 & -0.47779\end{array}$

$\begin{array}{llll}\text { C } & 2.29079 & 1.13081 & 0.28967\end{array}$

$\begin{array}{llll}H & 2.46926 & 2.16081 & -0.06409\end{array}$

$\begin{array}{llll}\text { C } & 1.31258 & -0.56410 & -2.86562\end{array}$

$\begin{array}{llll}\mathrm{H} & 2.22358 & -0.70451 & -3.46849\end{array}$

$\begin{array}{llll}\mathrm{H} & 0.53523 & -0.25455 & -3.57237\end{array}$

$\begin{array}{llll}\text { C } & 0.91790 & -1.91479 & -2.24933\end{array}$

H $\quad 1.79172 \quad-2.42137 \quad-1.83950$

$\begin{array}{llll}\mathrm{H} & 0.46850 & -2.59175 & -2.97586\end{array}$

$\begin{array}{llll}\text { C } & 0.36499 & 1.36311 & 1.78877\end{array}$

$\begin{array}{llll}\mathrm{H} & 1.05320 & 1.54744 & 2.61671\end{array}$

$\begin{array}{llll}\text { C } & -0.98792 & 1.51486 & 1.98247\end{array}$

$\begin{array}{llll}\mathrm{H} & -1.38555 & 1.83553 & 2.93700\end{array}$

$\begin{array}{llll}\text { C } & -0.00988 & 2.58270 & -1.25049\end{array}$

$\begin{array}{llll}\text { C } & 3.91147 & 0.26684 & -1.45538\end{array}$

$\begin{array}{llll}\text { C } & 4.34084 & 1.39121 & -2.15914\end{array}$

$\begin{array}{llll}\mathrm{H} & 3.63323 & 2.19123 & -2.35193\end{array}$

$\begin{array}{llll}\text { C } & 5.64880 & 1.48735 & -2.61056\end{array}$

$\begin{array}{llll}\mathrm{H} & 5.97028 & 2.36989 & -3.15169\end{array}$

$\begin{array}{llll}\text { C } & 6.54873 & 0.45533 & -2.36987\end{array}$

$\begin{array}{llll}\mathrm{H} & 7.56989 & 0.52947 & -2.72444\end{array}$

$\begin{array}{llll}\text { C } & 6.13022 & -0.67326 & -1.67891\end{array}$

$\begin{array}{llll}\text { H } & 6.82417 & -1.48479 & -1.49338\end{array}$

$\begin{array}{llll}\text { C } & 4.82023 & -0.76330 & -1.22561\end{array}$

$\begin{array}{llll}\mathrm{H} & 4.49173 & -1.64334 & -0.67852\end{array}$

$\begin{array}{llll}\text { C } & 3.25785 & 0.83352 & 1.40570\end{array}$

$\begin{array}{llll}\text { C } & 4.33000 & 1.68227 & 1.66592\end{array}$

$\begin{array}{llll}\mathrm{H} & 4.42838 & 2.60218 & 1.09864\end{array}$

$\begin{array}{llll}\text { C } & 5.27364 & 1.35761 & 2.63261\end{array}$

$\begin{array}{llll}\mathrm{H} & 6.10458 & 2.02728 & 2.82083\end{array}$

$\begin{array}{llll}\text { C } & 5.15297 & 0.17962 & 3.35647\end{array}$

$\begin{array}{llll}\mathrm{C} & 5.15297 & 0.17962 & 3.35647 \\ \mathrm{H} & 5.88738 & -0.07311 & 4.11184\end{array}$

$\begin{array}{llll}\text { C } & 4.08246 & -0.67173 & 3.10925\end{array}$

$\begin{array}{llll}\mathrm{H} & 3.97812 & -1.59363 & 3.67019\end{array}$

$\begin{array}{llll}\text { C } & 3.14592 & -0.34688 & 2.14044\end{array}$ 


$\begin{array}{llll}\text { H } & 2.31181 & -1.01138 & 1.94372 \\ \text { C } & -0.22197 & -1.97958 & 1.85145 \\ \text { H } & -0.97313 & -1.19771 & 1.86892 \\ \text { C } & 0.19295 & -2.56177 & 3.03958 \\ \text { H } & -0.24540 & -2.23944 & 3.97688 \\ \text { C } & 1.17754 & -3.54070 & 3.02583 \\ \text { H } & 1.51182 & -3.98992 & 3.95321 \\ \text { C } & 1.73435 & -3.94125 & 1.81868 \\ \text { H } & 2.49997 & -4.70764 & 1.80111 \\ \text { C } & 1.31179 & -3.36734 & 0.62713 \\ \text { H } & 1.75147 & -3.70269 & -0.30440 \\ \text { C } & 0.33242 & -2.37475 & 0.63348 \\ \text { C } & -1.76646 & -2.53548 & -1.33793 \\ \text { C } & -2.36513 & -2.27757 & -2.57459 \\ \text { H } & -1.94158 & -1.52729 & -3.23537 \\ \text { C } & -3.49653 & -2.97322 & -2.96811 \\ \text { H } & -3.94640 & -2.76676 & -3.93184 \\ \text { C } & -4.05289 & -3.93096 & -2.12857 \\ \text { H } & -4.93876 & -4.47376 & -2.43488 \\ \text { C } & -3.47234 & -4.18352 & -0.89476 \\ \text { H } & -3.90372 & -4.92472 & -0.23238 \\ \text { C } & -2.33628 & -3.49006 & -0.49798 \\ \text { H } & -1.89342 & -3.70154 & 0.46827 \\ \text { C } & -3.32613 & 0.11060 & 0.83615 \\ \text { C } & -3.56097 & -0.40548 & 2.10905 \\ \text { H } & -2.93893 & -0.08833 & 2.93943 \\ \text { C } & -4.57876 & -1.32659 & 2.31868 \\ \text { H } & -4.74755 & -1.72337 & 3.31283 \\ \text { C } & -5.38075 & -1.73313 & 1.26150 \\ \text { H } & -6.17669 & -2.44936 & 1.42673 \\ \text { C } & -5.15412 & -1.22603 & -0.01163 \\ \text { H } & -5.77057 & -1.54646 & -0.84305 \\ \text { C } & -4.12465 & -0.32207 & -0.22528 \\ \text { H } & -3.93904 & 0.05153 & -1.22726 \\ \text { C } & -2.92075 & 2.91083 & 0.34378 \\ \text { C } & -4.23107 & 2.99003 & -0.12663 \\ \text { H } & -4.78129 & 2.08952 & -0.36978 \\ \text { C } & -4.85686 & 4.22095 & -0.27266 \\ \text { H } & -5.87835 & 4.26121 & -0.63227 \\ \text { C } & -4.18455 & 5.39196 & 0.04491 \\ \text { H } & -4.67619 & 6.35078 & -0.06678 \\ \text { C } & -2.87901 & 5.32505 & 0.51315 \\ \text { H } & -2.34532 & 6.23252 & 0.76995 \\ \text { C } & -2.25414 & 4.09654 & 0.66081 \\ \text { H } & -1.23783 & 4.05058 & 1.03821\end{array}$

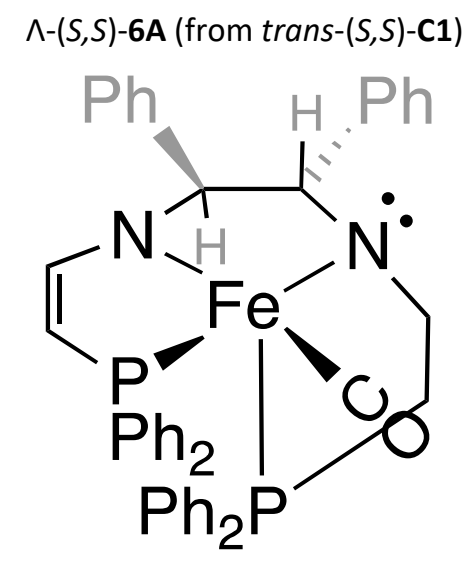

$\begin{array}{lccc}H^{\circ}= & -3792.267780 & & \\ \mathrm{G}^{\circ}= & -3792.389181 & & \\ \mathrm{Fe} & 0.14900 & 0.43384 & -0.88363 \\ \mathrm{P} & 0.57433 & -1.68379 & -0.61936 \\ \mathrm{P} & 1.69146 & 1.46117 & 0.41541 \\ \mathrm{O} & 1.81400 & 0.58344 & -3.26520 \\ \mathrm{~N} & -1.53477 & -0.23064 & -1.70513 \\ \mathrm{~N} & -1.03622 & 0.89798 & 0.59896 \\ \mathrm{C} & -2.67351 & 0.39980 & -1.00776 \\ \mathrm{H} & -2.65216 & 1.46350 & -1.28205 \\ \mathrm{C} & -2.40136 & 0.37389 & 0.50258 \\ \mathrm{H} & -2.40453 & -0.67374 & 0.84783 \\ \mathrm{C} & -1.72602 & -1.65901 & -1.93658 \\ \mathrm{H} & -2.31584 & -1.85010 & -2.84641 \\ \mathrm{H} & -2.29068 & -2.13802 & -1.12045 \\ \mathrm{C} & -0.38589 & -2.35476 & -2.03631 \\ \mathrm{H} & 0.14718 & -2.05952 & -2.94240 \\ \mathrm{H} & -0.46104 & -3.44346 & -2.03713 \\ \mathrm{C} & -0.57634 & 1.45843 & 1.72941 \\ \mathrm{H} & -1.28264 & 1.64199 & 2.54167 \\ \mathrm{C} & 0.73831 & 1.81054 & 1.87729 \\ \mathrm{H} & 1.10597 & 2.35847 & 2.73494 \\ \mathrm{C} & 1.15543 & 0.47573 & -2.30908 \\ \mathrm{C} & -4.02023 & -0.12487 & -1.44214 \\ \mathrm{C} & -4.58799 & 0.37788 & -2.61321 \\ \mathrm{H} & -4.06126 & 1.15238 & -3.16150 \\ \mathrm{C} & -5.80412 & -0.09508 & -3.08262 \\ \mathrm{H} & -6.22640 & 0.31174 & -3.99405 \\ \mathrm{C} & -6.48228 & -1.08404 & -2.38226 \\ \mathrm{H} & -7.43495 & -1.45317 & -2.74279 \\ \mathrm{C} & -5.93248 & -1.59274 & -1.21403 \\ \mathrm{H} & -6.45465 & -2.36376 & -0.65954 \\ \mathrm{C} & -4.71239 & -1.11920 & -0.75001 \\ \mathrm{H} & -4.29508 & -1.53499 & 0.16089 \\ \mathrm{C} & -3.42032 & 1.13238 & 1.30676 \\ \mathrm{C} & -3.69034 & 2.47764 & 1.04539 \\ \mathrm{H} & -3.13818 & 2.99268 & 0.26640 \\ \mathrm{C} & -4.64689 & 3.16343 & 1.77751 \\ \mathrm{H} & -4.84679 & 4.20592 & 1.55890 \\ \mathrm{C} & -5.34750 & 2.51993 & 2.79112 \\ \mathrm{H} & -6.09415 & 3.05736 & 3.36327 \\ \mathrm{C} & -5.07931 & 1.18751 & 3.06926 \\ \mathrm{H} & -5.61442 & 0.67952 & 3.86299 \\ \mathrm{C} & -4.12150 & 0.50261 & 2.33301\end{array}$




$\begin{array}{llll}\text { H } & -3.91002 & -0.53953 & 2.55307 \\ \text { C } & -0.17464 & -2.40503 & 0.88675 \\ \text { C } & 0.06539 & -1.77232 & 2.10711 \\ \text { H } & 0.71782 & -0.90571 & 2.14183 \\ \text { C } & -0.53967 & -2.22736 & 3.26747 \\ \text { H } & -0.34612 & -1.72518 & 4.20803 \\ \text { C } & -1.40067 & -3.31682 & 3.21936 \\ \text { H } & -1.88494 & -3.66668 & 4.12303 \\ \text { C } & -1.63785 & -3.95800 & 2.01200 \\ \text { H } & -2.30405 & -4.81130 & 1.97137 \\ \text { C } & -1.02446 & -3.51028 & 0.84897 \\ \text { H } & -1.21545 & -4.02604 & -0.08406 \\ \text { C } & 2.24086 & -2.43912 & -0.73378 \\ \text { C } & 2.91454 & -2.42778 & -1.95820 \\ \text { H } & 2.45873 & -1.97841 & -2.83209 \\ \text { C } & 4.17012 & -3.00339 & -2.07280 \\ \text { H } & 4.67723 & -2.99501 & -3.03001 \\ \text { C } & 4.77357 & -3.59019 & -0.96852 \\ \text { H } & 5.75496 & -4.03955 & -1.06012 \\ \text { C } & 4.11635 & -3.59493 & 0.25303 \\ \text { H } & 4.58338 & -4.04441 & 1.12120 \\ \text { C } & 2.85855 & -3.02161 & 0.37295 \\ \text { H } & 2.35419 & -3.03889 & 1.33200 \\ \text { C } & 2.34212 & 3.07950 & -0.18763 \\ \text { C } & 3.40850 & 3.72157 & 0.44345 \\ \text { H } & 3.91757 & 3.23949 & 1.27161 \\ \text { C } & 3.82312 & 4.97465 & 0.01853 \\ \text { H } & 4.65369 & 5.46351 & 0.51388 \\ \text { C } & 3.17774 & 5.60168 & -1.03994 \\ \text { H } & 3.50582 & 6.57890 & -1.37316 \\ \text { C } & 2.11387 & 4.97255 & -1.67069 \\ \text { H } & 1.60893 & 5.45635 & -2.49828 \\ \text { C } & 1.69965 & 3.71706 & -1.24834 \\ \text { H } & 0.86977 & 3.22378 & -1.74559 \\ \text { C } & 3.23293 & 0.59762 & 0.90244 \\ \text { C } & 3.43902 & 0.14633 & 2.20356 \\ \text { H } & 2.67689 & 0.31702 & 2.95645 \\ \text { C } & 4.61842 & -0.50708 & 2.54080 \\ \text { H } & 4.77153 & -0.84862 & 3.55768 \\ \text { C } & 5.59901 & -0.71286 & 1.58228 \\ \text { H } & 6.51872 & -1.22143 & 1.84559 \\ \text { C } & 5.39661 & -0.27251 & 0.27894 \\ \text { H } & 6.15733 & -0.43774 & -0.47480 \\ \text { C } & 4.21981 & 0.37409 & -0.06107 \\ \text { H } & 4.06893 & 0.71799 & -1.07989\end{array}$

$\Lambda-(S, S)-6 B$ (from trans-(S,S)-C1)

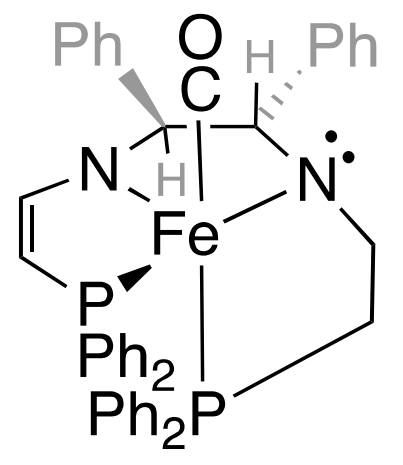

\begin{tabular}{|c|c|c|c|}
\hline \multicolumn{4}{|c|}{$H^{\circ}=-3792.246943$} \\
\hline & & & \\
\hline & 0.09338 & 0.60210 & -0.952 \\
\hline & 0.58718 & -1.68265 & -0.735 \\
\hline & 1.78111 & 1.38141 & 0.39665 \\
\hline & -0.52602 & 3.41579 & -1.47369 \\
\hline & -1.56748 & -0.11288 & -1.8117 \\
\hline & -1.00519 & 0.74484 & 0.63805 \\
\hline & -2.67283 & 0.47562 & -1.03586 \\
\hline & -2.63053 & 1.55888 & -1.20827 \\
\hline & -2.38203 & 0.28033 & 0.47169 \\
\hline & -2.40878 & -0.80283 & 0.679 \\
\hline & -1.75154 & -1.55324 & -1.9922 \\
\hline & -2.39418 & -1.75703 & -2.8622 \\
\hline & -2.26213 & -2.03330 & -1.140 \\
\hline & -0.41620 & -2.23439 & -2.1719 \\
\hline & 0.09326 & -1.87087 & -3.06807 \\
\hline & -0.49120 & -3.31985 & -2.25232 \\
\hline & -0.51735 & 1.30027 & 1.73367 \\
\hline & -1.18644 & 1.42844 & 2.5868 \\
\hline & 0.80103 & 1.70607 & 1.83490 \\
\hline & & 2.15 & 2.74420 \\
\hline & -0.25 & 2.29 & -1.2970 \\
\hline & -4.0 & 0.0 & -1.48218 \\
\hline & -4.62127 & 0.63568 & -2.5925 \\
\hline & -4.08629 & 1.44340 & -3.0821 \\
\hline & -5.85817 & 0.23449 & -3.0744 \\
\hline & -6.28 & 0.73068 & -3.9365 \\
\hline & -6.54681 & -0.79680 & -2.449 \\
\hline & -7.51542 & -1.11050 & -2.8195 \\
\hline & -5.9 & -1.4 & -1.3432 \\
\hline & -6.5 & -2.2 & -0.8477 \\
\hline & -4.7 & -1.0 & -0.8662 \\
\hline & -4.32284 & -1.5 & -0.0045 \\
\hline & -3.36954 & 0.95425 & 1.3830 \\
\hline & -3.60 & 2.32815 & 1.29171 \\
\hline & -3.0 & & 0.570 \\
\hline & -4.52607 & 2.94471 & 2.1220 \\
\hline & -4.69913 & 4.01117 & $2.037 \varepsilon$ \\
\hline & -5.22 & & 3.064 \\
\hline & -5. & 2.6 & 3.7150 \\
\hline & -4.9 & 6 & 3.17 \\
\hline & -5.5 & 33 & 3.9122 \\
\hline & -4.06964 & .22168 & 2.3385 \\
\hline & -3.88183 & -0.84451 & 425 \\
\hline & -0.12036 & -2.54196 & 0.711 \\
\hline & 0.05331 & -1.96619 & 1.9702 \\
\hline & 0.62555 & -1.05014 & 2.064 \\
\hline & -0.51616 & -2.54729 & 3.093 \\
\hline & -0.37721 & -2.08898 & 065 \\
\hline & -1.27178 & -3.70527 & 2.967 \\
\hline & & -4.15 & 201 \\
\hline & -1.44385 & -4.28843 & 1710 \\
\hline & -2.02737 & -5.19553 & 1010 \\
\hline & -0.86878 & -3.71332 & 0 \\
\hline & -1.01058 & -4.17946 & -0.372 \\
\hline & 2.23583 & -2.42631 & -1.0 \\
\hline
\end{tabular}




$\begin{array}{llll}\text { C } & 2.90986 & -2.09906 & -2.19584 \\ \text { H } & 2.46166 & -1.40763 & -2.90282 \\ \text { C } & 4.15060 & -2.65065 & -2.47044 \\ \text { H } & 4.66018 & -2.39614 & -3.39200 \\ \text { C } & 4.74102 & -3.52321 & -1.56448 \\ \text { H } & 5.71211 & -3.95266 & -1.77855 \\ \text { C } & 4.08798 & -3.83374 & -0.38083 \\ \text { H } & 4.54889 & -4.50394 & 0.33494 \\ \text { C } & 2.84105 & -3.28822 & -0.10437 \\ \text { H } & 2.33563 & -3.54206 & 0.82069 \\ \text { C } & 2.67528 & 2.92338 & -0.08878 \\ \text { C } & 3.21693 & 3.77165 & 0.87740 \\ \text { H } & 3.10661 & 3.53351 & 1.93005 \\ \text { C } & 3.89691 & 4.92009 & 0.50191 \\ \text { H } & 4.31037 & 5.57395 & 1.26070 \\ \text { C } & 4.05057 & 5.23180 & -0.84360 \\ \text { H } & 4.58207 & 6.12967 & -1.13500 \\ \text { C } & 3.52057 & 4.39156 & -1.81182 \\ \text { H } & 3.63709 & 4.63023 & -2.86230 \\ \text { C } & 2.83557 & 3.24386 & -1.43488 \\ \text { H } & 2.41665 & 2.59059 & -2.19460 \\ \text { C } & 3.23537 & 0.36132 & 0.91156 \\ \text { C } & 3.29384 & -0.22650 & 2.17242 \\ \text { H } & 2.48119 & -0.06671 & 2.87283 \\ \text { C } & 4.38887 & -0.99825 & 2.54226 \\ \text { H } & 4.42268 & -1.44451 & 3.52928 \\ \text { C } & 5.43722 & -1.19027 & 1.65522 \\ \text { H } & 6.29059 & -1.79326 & 1.94172 \\ \text { C } & 5.38847 & -0.60644 & 0.39434 \\ \text { H } & 6.20290 & -0.75548 & -0.30493 \\ \text { C } & 4.29639 & 0.16206 & 0.02504 \\ \text { H } & 4.27047 & 0.61601 & -0.96080\end{array}$

\begin{tabular}{|c|c|c|c|}
\hline & -2.52721 & 1801 & -0.01175 \\
\hline & -1.44094 & 0.51517 & -2.84584 \\
\hline & -2.40079 & 0.65635 & -3.35469 \\
\hline $\mathrm{H}$ & -0.71657 & 0.19236 & -3.59399 \\
\hline C & -0.99733 & 1.80655 & -2.18329 \\
\hline$H$ & -1.85086 & 2.33060 & -1.75300 \\
\hline$H$ & -0.55297 & 2.48012 & -2.91469 \\
\hline$C$ & -0.50455 & -1.51458 & 1.87194 \\
\hline $\mathrm{H}$ & -1.21297 & -1.76628 & 2.66607 \\
\hline$C$ & 0.84920 & -1.62789 & 2.10332 \\
\hline H & 1.24997 & -1.92624 & 3.06391 \\
\hline$C$ & 1.34413 & -0.96412 & -2.20862 \\
\hline C & -4.00058 & -0.46776 & -1.43513 \\
\hline C & -4.42305 & -1.64908 & -2.04465 \\
\hline $\mathrm{H}$ & -3.74139 & -2.49048 & -2.12372 \\
\hline C & -5.71131 & -1.76095 & -2.54560 \\
\hline $\mathrm{H}$ & -6.02840 & -2.68419 & -3.01548 \\
\hline C & -6.59556 & -0.69474 & -2.44113 \\
\hline H & -7.60237 & -0.78403 & -2.83068 \\
\hline C & -6.18426 & 0.48519 & -1.83734 \\
\hline $\mathrm{H}$ & -6.86899 & 1.32056 & -1.75458 \\
\hline C & -4.89335 & 0.59659 & -1.33958 \\
\hline $\mathrm{H}$ & -4.56938 & 1.51700 & -0.86307 \\
\hline C & -3.37200 & -1.00516 & 1.43730 \\
\hline C & -4.32963 & -1.94927 & 1.79250 \\
\hline $\mathrm{H}$ & -4.33394 & -2.91339 & 1.29454 \\
\hline C & -5.27530 & -1.66565 & 2.77065 \\
\hline $\mathrm{H}$ & -6.01508 & -2.41113 & 3.03725 \\
\hline C & -5.27061 & -0.43290 & 3.40695 \\
\hline $\mathrm{H}$ & -6.00595 & -0.21200 & 4.17127 \\
\hline C & -4.31530 & 0.5 & 112 \\
\hline $\mathrm{H}$ & -4.30305 & $1.4 \varepsilon$ & 3.55377 \\
\hline C & -3.37642 & 0.23117 & 2.08296 \\
\hline $\mathrm{H}$ & -2.63014 & 0.97090 & 1.81284 \\
\hline C & -0.11099 & 1.87132 & 1.90662 \\
\hline $\mathrm{H}$ & 0.46846 & 0.95924 & 1.99849 \\
\hline C & -0.54807 & 2.53378 & 3.04508 \\
\hline $\mathrm{H}$ & -0.29529 & 2.14405 & 4.02443 \\
\hline C & -1.32182 & 3.67983 & 2.92610 \\
\hline $\mathrm{H}$ & -1.67388 & 55 & 3.81259 \\
\hline C & -1.64244 & 4.16 & 1.66618 \\
\hline $\mathrm{H}$ & -2.23786 & 5.06924 & 1.56850 \\
\hline C & -1.19234 & 3.51632 & 0.52709 \\
\hline $\mathrm{H}$ & -1.43142 & 3.92676 & -0.44739 \\
\hline C & -0.42856 & 2.35394 & 0.63829 \\
\hline C & 1.70587 & 2.41835 & -1.26817 \\
\hline C & 2.24458 & 2.27528 & -2.54914 \\
\hline $\mathrm{H}$ & 1.75822 & 1.63087 & -3.27379 \\
\hline$r$ & 3.39559 & 2.95873 & -2.90904 \\
\hline $\mathrm{H}$ & 3.79816 & 2.84285 & -3.90820 \\
\hline C & 4.02731 & 3.79189 & -1.99419 \\
\hline $\mathrm{H}$ & 4.92653 & 4.32615 & -2.27583 \\
\hline C & 3.50154 & 3.93542 & -0.71882 \\
\hline $\mathrm{H}$ & 3.99023 & 4.58045 & 0.00187 \\
\hline C & 2.34908 & 3.25215 & -0.35436 \\
\hline $\mathrm{H}$ & 1.95229 & 3.37260 & 0.64729 \\
\hline C & 3.16963 & -0.15263 & 1.00293 \\
\hline C & 3.23781 & 0.55353 & 2.20058 \\
\hline
\end{tabular}

$\Delta$-(S,S)-G1A (from G1-1)

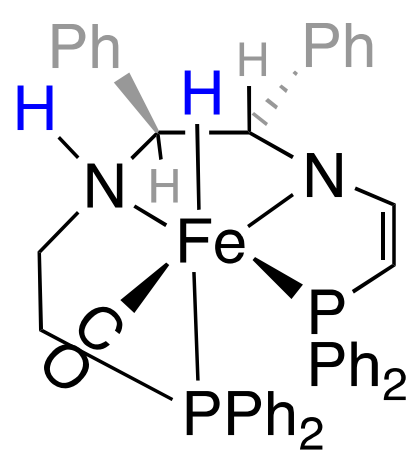

$\begin{array}{lccc}H^{\circ}=-3793.475113 & & \\ G^{\circ}= & -3793.597024 & & \\ \mathrm{Fe} & 0.26502 & -0.86344 & -0.85086 \\ \mathrm{H} & 0.06219 & -2.38585 & -1.03122 \\ \mathrm{P} & 0.22221 & 1.43441 & -0.81288 \\ \mathrm{P} & 1.82836 & -1.36618 & 0.64486 \\ \mathrm{O} & 2.08464 & -1.12712 & -3.09790 \\ \mathrm{~N} & -1.53348 & -0.57540 & -1.84746 \\ \mathrm{H} & -1.73388 & -1.43982 & -2.34398 \\ \mathrm{~N} & -0.98170 & -1.08039 & 0.70978 \\ \mathrm{C} & -2.61978 & -0.35060 & -0.85130 \\ \mathrm{H} & -2.48449 & 0.66781 & -0.47365 \\ \mathrm{C} & -2.37129 & -1.28363 & 0.34083\end{array}$




$\begin{array}{llll}\text { H } & 2.48945 & 0.37978 & 2.96743 \\ \mathrm{C} & 4.26475 & 1.46393 & 2.42465 \\ \mathrm{H} & 4.31083 & 2.00149 & 3.36462 \\ \mathrm{C} & 5.23227 & 1.67624 & 1.45382 \\ \mathrm{H} & 6.03254 & 2.38531 & 1.62907 \\ \mathrm{C} & 5.16966 & 0.97898 & 0.25215 \\ \mathrm{H} & 5.91921 & 1.14586 & -0.51256 \\ \mathrm{C} & 4.14510 & 0.07421 & 0.02758 \\ \mathrm{H} & 4.10647 & -0.47097 & -0.91087 \\ \mathrm{C} & 2.87635 & -2.87694 & 0.40355 \\ \mathrm{C} & 3.87559 & -3.18066 & 1.33241 \\ \mathrm{H} & 4.05861 & -2.51099 & 2.16697 \\ \mathrm{C} & 4.63842 & -4.33041 & 1.19968 \\ \mathrm{H} & 5.40619 & -4.55450 & 1.93092 \\ \mathrm{C} & 4.42323 & -5.19001 & 0.12911 \\ \mathrm{H} & 5.02291 & -6.08592 & 0.02209 \\ \mathrm{C} & 3.43841 & -4.89435 & -0.80131 \\ \mathrm{H} & 3.26755 & -5.55767 & -1.64111 \\ \mathrm{C} & 2.66777 & -3.74692 & -0.66300 \\ \mathrm{H} & 1.89243 & -3.52743 & -1.38721\end{array}$

$\begin{array}{llll}\mathrm{C} & 3.98917 & 0.36515 & -1.39572 \\ \mathrm{C} & 4.48600 & 1.50816 & -2.02152 \\ \mathrm{H} & 3.84735 & 2.37666 & -2.15141 \\ \mathrm{C} & 5.79562 & 1.54895 & -2.47654 \\ \mathrm{H} & 6.17023 & 2.44342 & -2.95960 \\ \mathrm{C} & 6.62747 & 0.44939 & -2.30884 \\ \mathrm{H} & 7.65091 & 0.48370 & -2.66193 \\ \mathrm{C} & 6.14195 & -0.69357 & -1.68911 \\ \mathrm{H} & 6.78487 & -1.55548 & -1.55720 \\ \mathrm{C} & 4.83004 & -0.73406 & -1.23812 \\ \mathrm{H} & 4.44868 & -1.62602 & -0.74968 \\ \mathrm{C} & 3.33804 & 0.96578 & 1.44813\end{array}$

$\begin{array}{llll}C & 4.29984 & 1.89596 & 1.82747\end{array}$

$\begin{array}{llll}\mathrm{H} & 4.32389 & 2.86369 & 1.33712\end{array}$

$\begin{array}{llll}\text { C } & 5.22541 & 1.59361 & 2.81930\end{array}$

$\begin{array}{llll}\mathrm{H} & 5.96905 & 2.32826 & 3.10455\end{array}$

$\begin{array}{llll}\text { C } & 5.19583 & 0.35599 & 3.44541\end{array}$

$\begin{array}{llll}\mathrm{H} & 5.91518 & 0.12070 & 4.22060\end{array}$

$\begin{array}{llll}\text { C } & 4.23643 & -0.57974 & 3.07503\end{array}$

$\begin{array}{llll}\mathrm{H} & 4.20458 & -1.54954 & 3.55865\end{array}$

$\Delta-(S, S)-$ G1B (from G1-1)

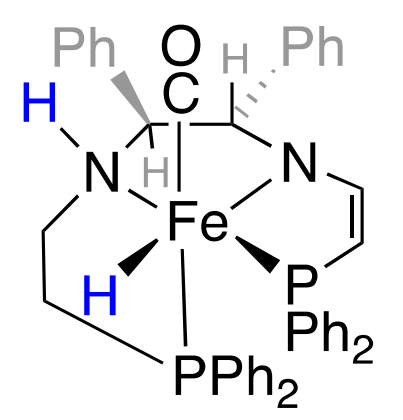

$\begin{array}{lccc}H^{\circ}=-3793.467426 & & \\ G^{\circ}=-3793.591170 & & \\ \mathrm{Fe} & -0.27242 & 0.91534 & -0.88200 \\ \mathrm{H} & -1.15863 & 0.62337 & -2.13686 \\ \mathrm{P} & -0.27766 & -1.38865 & -0.94136 \\ \mathrm{P} & -1.88285 & 1.24492 & 0.64832 \\ \mathrm{O} & -0.42712 & 3.69104 & -1.72387 \\ \mathrm{~N} & 1.53877 & 0.61079 & -1.87081 \\ \mathrm{H} & 1.78944 & 1.48118 & -2.33373 \\ \mathrm{~N} & 0.95874 & 1.10220 & 0.69168 \\ \mathrm{C} & 2.58844 & 0.32223 & -0.85092 \\ \mathrm{H} & 2.38969 & -0.68859 & -0.48135 \\ \mathrm{C} & 2.35650 & 1.26595 & 0.34102 \\ \mathrm{H} & 2.54518 & 2.29446 & -0.01578 \\ \mathrm{C} & 1.43613 & -0.43360 & -2.91478 \\ \mathrm{H} & 2.40281 & -0.58187 & -3.40866 \\ \mathrm{H} & 0.73664 & -0.05764 & -3.66011 \\ \mathrm{C} & 0.94217 & -1.73514 & -2.31679 \\ \mathrm{H} & 1.77152 & -2.30632 & -1.89950 \\ \mathrm{H} & 0.48222 & -2.35962 & -3.08117 \\ \mathrm{C} & 0.46498 & 1.47253 & 1.86464 \\ \mathrm{H} & 1.15807 & 1.70231 & 2.67908 \\ \mathrm{C} & -0.89652 & 1.54239 & 2.09430 \\ \mathrm{H} & -1.29283 & 1.75284 & 3.07947 \\ \mathrm{C} & -0.35571 & 2.58992 & -1.34764\end{array}$

$\begin{array}{llll}\text { C } & 3.31818 & -0.27601 & 2.08311\end{array}$

$\begin{array}{llll}\mathrm{H} & 2.57111 & -1.00722 & 1.79272\end{array}$

$\begin{array}{llll}\text { C } & 0.08602 & -1.93687 & 1.75530\end{array}$

H $\quad-0.44441 \quad-1.00119 \quad 1.88980$

$\begin{array}{llll}\text { C } & 0.50730 & -2.65865 & 2.86365\end{array}$

$\begin{array}{llll}\mathrm{H} & 0.28759 & -2.29110 & 3.85961\end{array}$

$\begin{array}{llll}\text { C } & 1.22488 & -3.83383 & 2.69321\end{array}$

$\begin{array}{llll}\text { H } & 1.56495 & -4.39458 & 3.55558\end{array}$

$\begin{array}{llll}\text { C } & 1.50564 & -4.29158 & 1.41160\end{array}$

H $\quad 2.05883 \quad-5.21284 \quad 1.27331$

$\begin{array}{llll}\text { C } & 1.06834 & -3.57990 & 0.30341\end{array}$

H $\quad 1.27321 \quad-3.96776 \quad-0.68795$

$\begin{array}{llll}\text { C } & 0.35854 & -2.38895 & 0.46602\end{array}$

$\begin{array}{llll}\text { C } & -1.78452 & -2.32164 & -1.43269\end{array}$

$\begin{array}{llll}\text { C } & -2.40292 & -2.01605 & -2.64703\end{array}$

$\begin{array}{llll}\mathrm{H} & -1.98386 & -1.24547 & -3.28552\end{array}$

$\begin{array}{llll}\text { C } & -3.54893 & -2.68750 & -3.04215\end{array}$

$\begin{array}{llll}\mathrm{C} & -3.54893 & -2.68750 & -3.04215 \\ \mathrm{H} & -4.01445 & -2.44297 & -3.98941\end{array}$

$\begin{array}{llll}\text { C } & -4.09874 & -3.66939 & -2.22713\end{array}$

H $\quad-4.99641 \quad-4.19176 \quad-2.53495$

$\begin{array}{llll}\text { C } & -3.49551 & -3.97328 & -1.01627\end{array}$

H $\quad-3.92280 \quad-4.73095 \quad-0.37006$

$\begin{array}{llll}\text { C } & -2.34513 & -3.30450 & -0.61873\end{array}$

$\begin{array}{llll}H & -1.89037 & -3.55074 & 0.33353\end{array}$

$\begin{array}{llll}H & -1.89037 & -3.55074 & 0.33353 \\ C & -3.16443 & -0.02934 & 1.04606\end{array}$

$\begin{array}{llll}\text { C } & -3.15543 & -0.71628 & 2.25756\end{array}$

$\begin{array}{llll}\text { H } & -2.38779 & -0.49577 & 2.99198\end{array}$

$\begin{array}{llll}\text { C } & -4.12498 & -1.67255 & 2.53491\end{array}$

$\begin{array}{llll}\mathrm{H} & -4.10608 & -2.19687 & 3.48327\end{array}$

$\begin{array}{llll}\text { C } & -5.11706 & -1.94932 & 1.60595\end{array}$

$\begin{array}{llll}\mathrm{C} & -5.11706 & -1.94932 & 1.60595 \\ \mathrm{H} & -5.87368 & -2.69403 & 1.82241\end{array}$

$\begin{array}{llll}H & -5.87368 & -2.69403 & 1.82241 \\ \text { C } & -5.13583 & -1.26773 & 0.39445\end{array}$

H $\quad-5.90420 \quad-1.48368 \quad-0.33868$

$\begin{array}{llll}\text { C } & -4.16462 & -0.31997 & 0.11420\end{array}$

$\begin{array}{llll}\mathrm{H} & -4.18278 & 0.19825 & -0.83926\end{array}$

$\begin{array}{llll}\text { C } & -3.00978 & 2.69241 & 0.39951\end{array}$

$\begin{array}{llll}\text { C } & -3.36049 & 3.51520 & 1.46826\end{array}$

$\begin{array}{llll}\text { H } & -2.95811 & 3.31607 & 2.45546\end{array}$ 


$\begin{array}{llll}\mathrm{C} & -4.21215 & 4.59432 & 1.27717 \\ \mathrm{H} & -4.47365 & 5.22854 & 2.11616 \\ \mathrm{C} & -4.72609 & 4.86229 & 0.01556 \\ \mathrm{H} & -5.38767 & 5.70712 & -0.13370 \\ \mathrm{C} & -4.38265 & 4.04829 & -1.05536 \\ \mathrm{H} & -4.77415 & 4.25667 & -2.04411 \\ \mathrm{C} & -3.52846 & 2.97140 & -0.86446 \\ \mathrm{H} & -3.24685 & 2.34981 & -1.70882\end{array}$

$\wedge$-(S,S)-G1A (from G1-1)

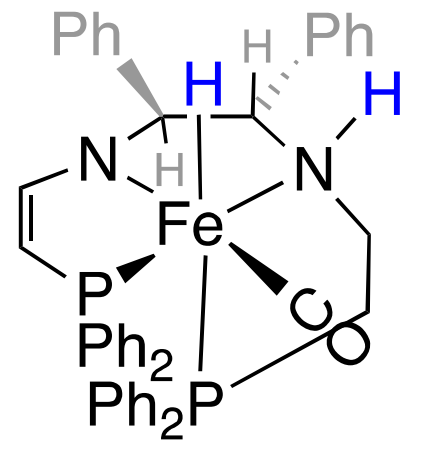

\begin{tabular}{|c|c|c|c|}
\hline$C$ & -3.69667 & 2.40447 & 1.19633 \\
\hline $\mathrm{H}$ & -3.17061 & 2.97710 & 0.43926 \\
\hline$C$ & -4.61719 & 3.03410 & 2.01929 \\
\hline $\mathrm{H}$ & -4.81813 & 4.09135 & 1.89300 \\
\hline$C$ & -5.27970 & 2.31423 & 3.00670 \\
\hline $\mathrm{H}$ & -5.99843 & 2.80769 & 3.64978 \\
\hline$C$ & -5.01227 & 0.96274 & 3.16736 \\
\hline $\mathrm{H}$ & -5.51947 & 0.39579 & 3.93897 \\
\hline$C$ & -4.09061 & 0.33448 & 2.33970 \\
\hline $\mathrm{H}$ & -3.87902 & -0.72317 & 2.46457 \\
\hline$C$ & -0.19859 & -2.44160 & 0.86267 \\
\hline$C$ & -0.00159 & -1.81882 & 2.09574 \\
\hline $\mathrm{H}$ & 0.60182 & -0.91795 & 2.14618 \\
\hline$C$ & -0.58025 & -2.33255 & 3.24660 \\
\hline $\mathrm{H}$ & -0.41906 & -1.83782 & 4.19730 \\
\hline$C$ & -1.37504 & -3.46893 & 3.17565 \\
\hline $\mathrm{H}$ & -1.83796 & -3.86559 & 4.07124 \\
\hline$C$ & -1.57645 & -4.09627 & 1.95348 \\
\hline $\mathrm{H}$ & -2.19092 & -4.98665 & 1.89454 \\
\hline$C$ & -0.98843 & -3.58961 & 0.80250 \\
\hline $\mathrm{H}$ & -1.15105 & -4.09731 & -0.14094 \\
\hline$C$ & 2.16256 & -2.50501 & -0.80705 \\
\hline$C$ & 2.83684 & -2.42643 & -2.02889 \\
\hline $\mathrm{H}$ & 2.40633 & -1.87486 & -2.85674 \\
\hline $\mathrm{C}$ & 4.05949 & -3.05719 & -2.19851 \\
\hline $\mathrm{H}$ & 4.56648 & -2.99399 & -3.15393 \\
\hline$C$ & 4.63259 & -3.76554 & -1.15021 \\
\hline $\mathrm{H}$ & 5.58848 & -4.25735 & -1.28400 \\
\hline$C$ & 3.97698 & -3.83540 & 0.07003 \\
\hline $\mathrm{H}$ & 4.42108 & -4.37782 & 0.89634 \\
\hline$C$ & 2.75040 & -3.20890 & 0.24310 \\
\hline $\mathrm{H}$ & 2.24734 & -3.27681 & 1.20085 \\
\hline $\mathrm{H}$ & -0.26041 & 2.01503 & -1.33137 \\
\hline$C$ & 2.53263 & 3.08025 & -0.05022 \\
\hline$C$ & 3.51678 & 3.61436 & 0.78587 \\
\hline $\mathrm{H}$ & 3.80875 & 3.08130 & 1.68521 \\
\hline$C$ & 4.12599 & 4.82106 & 0.47910 \\
\hline $\mathrm{H}$ & 4.88365 & 5.22536 & 1.14007 \\
\hline$C$ & 3.77018 & 5.50748 & -0.67547 \\
\hline $\mathrm{H}$ & 4.25101 & 6.44732 & -0.91883 \\
\hline$C$ & 2.79872 & 4.98292 & -1.51503 \\
\hline $\mathrm{H}$ & 2.51947 & 5.51116 & -2.41913 \\
\hline$C$ & 2.18159 & 3.77835 & -1.20261 \\
\hline $\mathrm{H}$ & 1.41596 & 3.37713 & -1.85649 \\
\hline$C$ & 3.16408 & 0.51208 & 0.90411 \\
\hline$C$ & 3.30577 & -0.01620 & 2.18412 \\
\hline $\mathrm{H}$ & 2.53655 & 0.17419 & 2.92580 \\
\hline$C$ & 4.42678 & -0.76725 & 2.51854 \\
\hline $\mathrm{H}$ & 4.52804 & -1.16581 & 3.52136 \\
\hline$C$ & 5.41603 & -0.99911 & 1.57462 \\
\hline $\mathrm{H}$ & 6.28956 & -1.58560 & 1.83356 \\
\hline$C$ & 5.27991 & -0.48233 & 0.29068 \\
\hline $\mathrm{H}$ & 6.04721 & -0.66597 & -0.45245 \\
\hline$C$ & 4.16176 & 0.26513 & -0.04256 \\
\hline $\mathrm{H}$ & 4.06474 & 0.66837 & -1.04647 \\
\hline
\end{tabular}

$\begin{array}{lccc}H^{\circ}= & -3793.470143 & & \\ \mathrm{G}^{\circ}= & -3793.591878 & & \\ \mathrm{Fe} & 0.18449 & 0.58341 & -0.94376 \\ \mathrm{P} & 0.55072 & -1.64802 & -0.61125 \\ \mathrm{P} & 1.68345 & 1.50308 & 0.41959 \\ \mathrm{O} & 1.86628 & 0.79794 & -3.29917 \\ \mathrm{~N} & -1.62116 & -0.10328 & -1.80783 \\ \mathrm{H} & -1.63912 & 0.30071 & -2.73941 \\ \mathrm{~N} & -1.04827 & 0.81201 & 0.62917 \\ \mathrm{C} & -2.73286 & 0.53996 & -1.03028 \\ \mathrm{H} & -2.62905 & 1.60533 & -1.24855 \\ \mathrm{C} & -2.41703 & 0.35398 & 0.45919 \\ \mathrm{H} & -2.46294 & -0.72556 & 0.68661 \\ \mathrm{C} & -1.79065 & -1.57788 & -1.95579 \\ \mathrm{H} & -2.40920 & -1.80006 & -2.82821 \\ \mathrm{H} & -2.33487 & -1.94457 & -1.08346 \\ \mathrm{C} & -0.45178 & -2.27152 & -2.03415 \\ \mathrm{H} & 0.08166 & -1.99248 & -2.94547 \\ \mathrm{H} & -0.56534 & -3.35641 & -2.04270 \\ \mathrm{C} & -0.61160 & 1.40418 & 1.72828 \\ \mathrm{H} & -1.30762 & 1.56623 & 2.55458 \\ \mathrm{C} & 0.70106 & 1.81654 & 1.85909 \\ \mathrm{H} & 1.05445 & 2.34456 & 2.73564 \\ \mathrm{C} & 1.19620 & 0.65933 & -2.35185 \\ \mathrm{C} & -4.10291 & 0.09611 & -1.48009 \\ \mathrm{C} & -4.68765 & 0.73138 & -2.57552 \\ \mathrm{H} & -4.15829 & 1.54748 & -3.05695 \\ \mathrm{C} & -5.93018 & 0.33864 & -3.04994 \\ \mathrm{H} & -6.36797 & 0.84683 & -3.90081 \\ \mathrm{C} & -6.61410 & -0.69829 & -2.43005 \\ \mathrm{H} & -7.58696 & -1.00465 & -2.79507 \\ \mathrm{C} & -6.04648 & -1.33659 & -1.33634 \\ \mathrm{H} & -6.57514 & -2.14467 & -0.84503 \\ & -4.80119 & -0.94421 & -0.86642 \\ \mathrm{C} & -4.37253 & -1.45697 & -0.01232 \\ & & & \\ \mathrm{H} & -32849 & 1.04284 & 1.34160\end{array}$


$\Lambda$-(S,S)-G1B (from G1-1)

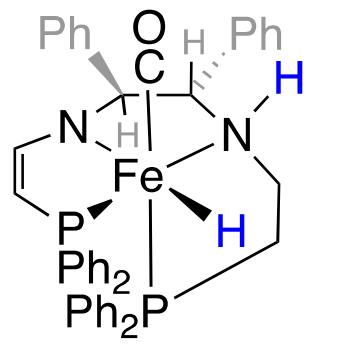

$H^{\circ}=-3793.463193$

$G^{\circ}=-3793.585392$

Fe $\quad 0.17827 \quad 0.60461$

$\begin{array}{lll}P & 0.60577 & -1.63327\end{array}$

$\begin{array}{lll}P & 1.73796 \quad 1.38999\end{array}$

O $\quad-0.17978 \quad 3.23313$

N $\quad-1.62360 \quad-0.11445$

$\begin{array}{lll}\mathrm{H} & -1.66618 & 0.30327\end{array}$

N $\quad-1.03525 \quad 0.78636$

C $\quad-2.73383 \quad 0.49854$

H $\quad-2.65616 \quad 1.56998$

$\begin{array}{lll}\text { C } & -2.39324 & 0.29757\end{array}$

H $\quad-2.40952-0.78702$

C $\quad-1.76906 \quad-1.58672$

H $\quad-2.41629 \quad-1.80077$

H $\quad-2.26888 \quad-1.98853$

C $\quad-0.41595 \quad-2.23610$

H $\quad 0.08230 \quad-1.90783$

H $\quad-0.49450 \quad-3.32323$

$\begin{array}{lll}\text { C } & -0.57357 & 1.31307\end{array}$

H $\quad-1.25447 \quad 1.44224$

C $\quad 0.74973 \quad 1.69795$

H 1.11468

C $\quad-0.04581$

C -4.10226

2.14075

2.19431

0.03685

0.68110

C $\quad-4.71763$

H $\quad-4.21328$

C $\quad-5.95928$

1.51752

0.27152

0.78707

H $\quad-6.42160$

C $\quad-6.61076$

H $\quad-7.58274$

C $\quad-6.01207$

$-0.79203$

$-1.11186$

$-1.43971$

H $\quad-6.51542 \quad-2.26864$

C $\quad-4.76788 \quad-1.03030$

H $\quad-4.31417 \quad-1.55070$

$\begin{array}{lll}\text { C } & -3.40544 & 0.94582\end{array}$

$\begin{array}{lll}\text { C } & -3.70954 & 2.30267\end{array}$

H $\quad-3.21605 \quad 2.90066$

C $\quad-4.62413 \quad 2.89589$

H $\quad-4.85371 \quad 3.94984$

C $\quad-5.24318 \quad 2.14373$

H $\quad-5.95662 \quad 2.60880$

C $\quad-4.93876 \quad 0.79684$

H $\quad-5.41180 \quad 0.20553$

C $\quad-4.02403 \quad 0.20494$

H $\quad-3.78181-0.84870$

$\begin{array}{lll}\text { C } & -0.08075 & -2.53297\end{array}$

C $\quad 0.06707 \quad-1.94083$
$-0.95495$

$-0.72947$

0.45534

$-2.14274$

$-1.81833$

$-2.74346$

0.63420

$-1.00969$

$-1.21443$

0.47340

0.68164

$-1.99666$

$-2.85015$

$-1.11315$

$-2.14494$

$-3.05853$

$-2.18338$

1.75463

2.60014

1.89212

2.80973

$-1.63108$

$-1.44473$

$-2.51767$

$-2.99114$

$-2.97999$

$-3.81324$

$-2.37047$

$-2.72616$

$-1.29903$

$-0.81593$

$-0.84097$

$-0.00464$

1.38461

1.26957

0.50986

2.12586

2.02277

3.11736

3.78693

3.24852

4.02352

2.38709

2.48917

0.71704

1.97137
政

\section{(c)}

\section{政}

\section{促}

年

年

tid

c

H 4.54117

C 2.84392

H 2.34600

H $\quad 1.09068$

C 2.63526

C 2.97313

$\mathrm{H} \quad 2.69144$

C $\quad 3.66366$

H $\quad 3.91758$

C 4.02900

H 4.56600

C $\quad 3.69978$

H 3.97835

C $\quad 3.00716$

$\begin{array}{ll}\mathrm{H} & 2.73981\end{array}$

C $\quad 3.20262$

C $\quad 3.28452$

H 2.48197

C 4.38858

H 4.44084

$\mathrm{H}$
$\mathrm{C}$

H 6.28349

C $\quad 5.34915$

H 6.15094

C 4.24784

H 4.19942

$-1.00068$

2.05506

3.10162

$-2.53188$

4.06891

2.98858

$-3.71785$

$\begin{array}{ll}-4.17553 & 3.86754 \\ -4.31640 & 1.74477\end{array}$

$-4.31640$

1.65253

$-3.73162$

0.61504

$-4.21630$

$-0.34604$

$-2.40639-1.01868$

$-2.14187-2.21755$

$-1.47519-2.94690$

$-2.71948$

$-2.47865$

$-2.51143$

$-3.41547$

$-3.55852-1.54166$

$-4.00656$

$-1.74455$

$-3.81387$

$-0.34254$

$-4.45884$

$-3.24312$

0.39762

$-3.45324$

0.85941

0.31123

2.94099

$-2.19035$

$-0.00353$

3.88076

0.96944

3.71255

2.00336

5.03395

0.62567

5.75783

1.39109

5.26048

6.16238

$-0.69494$

4.32964

$-0.96310$

4.50287

$-1.67029$

3.17678

$-2.70312$

2.45683

$-1.32623$

0.37239

$-2.09343$

0.95303

$-0.20649$

2.21680

$-0.04607$

2.92900

$-0.97164$

2.57396

$-1.41030$

3.56369

$-1.16634$

1.67088

$-1.76337$

1.94803

$-0.59481 \quad 0.40551$

$-0.74858$

$-0.30731$

0.16676

0.04904

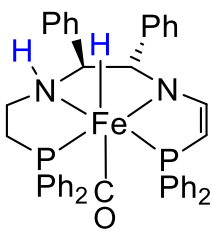

0.60629

$-0.94230$

\section{G1-1}

G1-1 from C1 (see complex $\mathbf{5}$ in W. Zuo, D. E. Prokopchuk, A. J. Lough, R. H. Morris, ACS Catalysis 2016, 6, 301-314)

$G^{\circ}=-3793.467893$

$H^{\circ}=-3793.59166$

$\begin{array}{lrrr}C & 2.97864 & 0.62549 & -0.82895 \\ C & 2.97692 & -0.66916 & 0.01503\end{array}$ 


\begin{tabular}{|c|c|c|c|}
\hline $\mathrm{H}$ & 2.20712 & 3.12685 & -1.27713 \\
\hline $\mathrm{N}$ & 1.65896 & 1.20425 & -0.70005 \\
\hline$C$ & 1.38634 & 2.46580 & -0.98648 \\
\hline $\mathrm{N}$ & 1.71711 & -1.38328 & -0.29796 \\
\hline $\mathrm{H}$ & 0.13619 & -3.51617 & -0.89350 \\
\hline C & 0.11277 & -3.14948 & 0.13473 \\
\hline $\mathrm{Fe}$ & 0.17528 & -0.01769 & -0.15259 \\
\hline $\mathrm{H}$ & -0.16226 & -3.99042 & 0.77058 \\
\hline$P$ & -1.13823 & 1.76224 & -0.50773 \\
\hline$P$ & -1.13561 & -1.76880 & 0.22296 \\
\hline C & 0.44591 & 0.37003 & 1.55529 \\
\hline 0 & 0.64624 & 0.65779 & 2.66567 \\
\hline C & 1.46887 & -2.59189 & 0.51046 \\
\hline $\mathrm{H}$ & 1.49829 & -2.29279 & 1.56207 \\
\hline $\mathrm{H}$ & 2.25405 & -3.33903 & 0.35536 \\
\hline C & 0.10093 & 2.96718 & -0.91939 \\
\hline $\mathrm{H}$ & -0.12285 & 4.00202 & -1.14757 \\
\hline C & -2.44886 & -2.25399 & -0.96998 \\
\hline C & -3.79414 & -2.04267 & -0.66821 \\
\hline $\mathrm{C}$ & -2.10948 & -2.71660 & -2.24244 \\
\hline C & -4.77800 & -2.30091 & -1.61279 \\
\hline $\mathrm{H}$ & -4.07950 & -1.67288 & 0.31057 \\
\hline C & -3.09441 & -2.98498 & -3.18060 \\
\hline $\mathrm{H}$ & -1.06854 & -2.85496 & -2.51317 \\
\hline C & -4.43174 & -2.77704 & -2.86881 \\
\hline $\mathrm{H}$ & -5.81826 & -2.12980 & -1.36311 \\
\hline $\mathrm{H}$ & -2.81485 & -3.35000 & -4.16167 \\
\hline $\mathrm{H}$ & -5.19986 & -2.98035 & -3.60503 \\
\hline C & -1.98608 & -2.03223 & 1.83556 \\
\hline C & -2.39716 & -3.30611 & 2.23451 \\
\hline C & -2.28360 & -0.94327 & 2.65069 \\
\hline C & -3.07369 & -3.48366 & 3.43078 \\
\hline $\mathrm{H}$ & -2.19934 & -4.16582 & 1.60344 \\
\hline C & -2.96882 & -1.11990 & 3.84586 \\
\hline $\mathrm{H}$ & -1.98950 & 0.05360 & 2.34477 \\
\hline C & -3.35983 & -2.39044 & 4.23968 \\
\hline $\mathrm{H}$ & -3.38353 & -4.47747 & 3.73100 \\
\hline $\mathrm{H}$ & -3.19599 & -0.26031 & 4.46525 \\
\hline $\mathrm{H}$ & -3.89076 & -2.53144 & 5.17341 \\
\hline C & -2.42453 & 1.84934 & -1.83441 \\
\hline C & -3.20320 & 3.00404 & -1.95960 \\
\hline C & -2.59514 & 0.82490 & -2.76051 \\
\hline C & -4.12807 & 3.12547 & -2.98425 \\
\hline $\mathrm{H}$ & -3.08532 & 3.81531 & -1.24865 \\
\hline C & -3.52705 & 0.94333 & -3.78438 \\
\hline $\mathrm{H}$ & -1.98849 & -0.06954 & -2.68008 \\
\hline C & -4.29559 & 2.09216 & -3.89856 \\
\hline $\mathrm{H}$ & -4.72104 & 4.02850 & -3.06993 \\
\hline $\mathrm{H}$ & -3.64908 & 0.13462 & -4.49571 \\
\hline $\mathrm{H}$ & -5.02165 & 2.18524 & -4.69731 \\
\hline C & -2.10485 & 2.39391 & 0.93239 \\
\hline C & -1.46128 & 3.14347 & 1.91545 \\
\hline C & -3.43448 & 2.01874 & 1.13630 \\
\hline C & -2.12895 & 3.51224 & 3.07510 \\
\hline $\mathrm{H}$ & -0.42645 & 3.43677 & 1.76462 \\
\hline C & -4.10306 & 2.38863 & 2.29389 \\
\hline $\mathrm{H}$ & -3.94954 & 1.42493 & 0.38777 \\
\hline C & -3.45111 & 3.13424 & 3.26797 \\
\hline
\end{tabular}

$\begin{array}{lrrr}\mathrm{H} & -1.61588 & 4.09715 & 3.82950 \\ \mathrm{H} & -5.13471 & 2.08967 & 2.43880 \\ \mathrm{H} & -3.97324 & 3.42200 & 4.17257 \\ \mathrm{H} & 3.16587 & 0.31621 & -1.87269 \\ \mathrm{H} & 2.90765 & -0.38290 & 1.06930 \\ \mathrm{C} & 4.11754 & 1.51763 & -0.39365 \\ \mathrm{C} & 5.27710 & 1.61827 & -1.15575 \\ \mathrm{C} & 4.04887 & 2.20141 & 0.82040 \\ \mathrm{C} & 6.35173 & 2.37973 & -0.71399 \\ \mathrm{H} & 5.33729 & 1.08857 & -2.10116 \\ \mathrm{C} & 5.11907 & 2.96267 & 1.26292 \\ \mathrm{H} & 3.14407 & 2.13482 & 1.41689 \\ \mathrm{C} & 6.27574 & 3.05213 & 0.49719 \\ \mathrm{H} & 7.24867 & 2.44876 & -1.31805 \\ \mathrm{H} & 5.05283 & 3.48840 & 2.20815 \\ \mathrm{H} & 7.11172 & 3.64847 & 0.84233 \\ \mathrm{C} & 4.22378 & -1.48785 & -0.18934 \\ \mathrm{C} & 5.20405 & -1.52645 & 0.79867 \\ \mathrm{C} & 4.44623 & -2.17130 & -1.38386 \\ \mathrm{C} & 6.38608 & -2.22520 & 0.59693 \\ \mathrm{H} & 5.03855 & -0.99435 & 1.72986 \\ \mathrm{C} & 5.62369 & -2.87605 & -1.58522 \\ \mathrm{H} & 3.69322 & -2.15264 & -2.16560 \\ \mathrm{C} & 6.59868 & -2.90138 & -0.59626 \\ \mathrm{H} & 7.14148 & -2.24244 & 1.37341 \\ \mathrm{H} & 5.78280 & -3.40426 & -2.51773 \\ \mathrm{H} & 7.51964 & -3.44913 & -0.75523 \\ \mathrm{H} & -0.10476 & -0.31624 & -1.68288 \\ \mathrm{H} & 1.74496 & -1.66110 & -1.2780\end{array}$


